\title{
Mechanisms underlying the nuclear transport of histones and histone-related proteins
}

\author{
Dissertation \\ zur Erlangung des Doktorgrades \\ der Mathematisch-Naturwissenschaftlichen Fakultäten \\ der Georg-August-Universität zu Göttingen
}

vorgelegt von

Jörg Kahle

aus Dresden

Göttingen 2005 
D7

Referent:

Prof. Dr. D. Doenecke

Korreferent:

Prof. Dr. R. Ficner

Tag der mündlichen Prüfung:

27. April 2005 
Die vorliegende Arbeit wurde in der Zeit von Oktober 2001 bis März 2005 in der Abteilung Molekularbiologie unter Anleitung von Prof. Dr. D. Doenecke am Institut für Biochemie und Molekulare Zellbiologie der Georg-August-Universität zu Göttingen angefertigt.

Regardless of the fact that the data presented in this work was essentially obtained, evaluated and put to paper by myself, I would like to use the term "We" instead of "I" because of the guidance and advice given to me by my supervisors (and others) and because of the daily support received from my colleagues regarding the bench work (thanks). 
In Memory of

Edna Bodden

Falko Rudt

Kurt Jungermann 


\section{Contents}

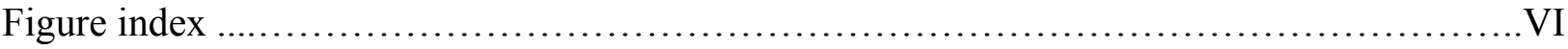

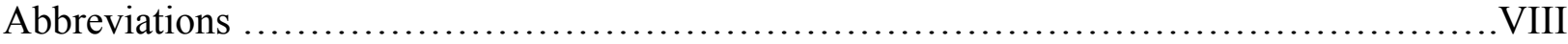

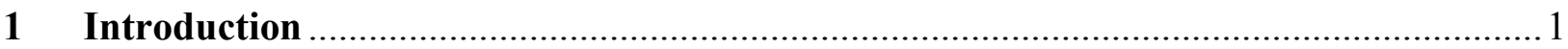

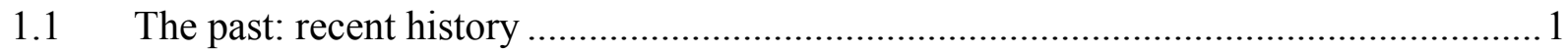

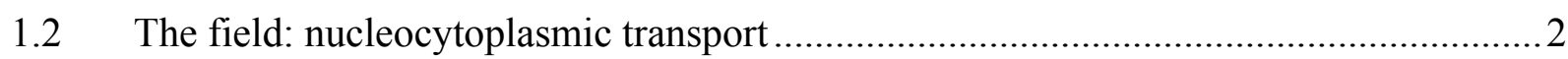

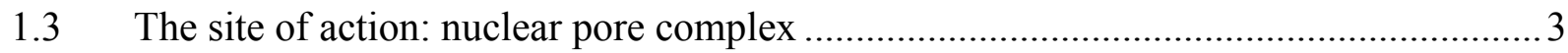

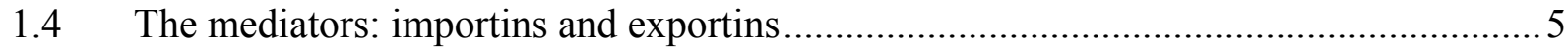

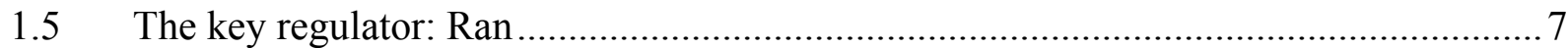

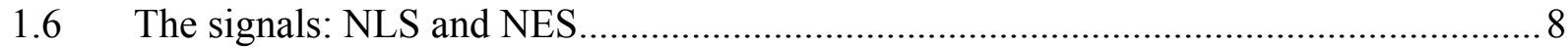

1.6.1 The admission ticket for the nucleus: NLSs................................................... 10

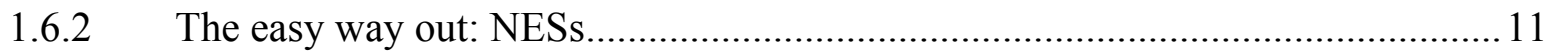

1.7 Regulated nuclear transport: rule or exception? ..................................................... 13

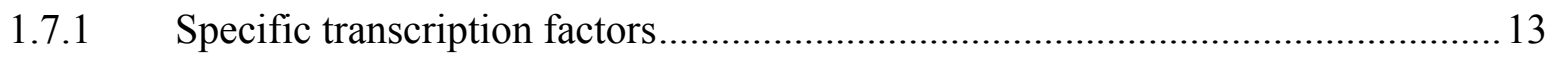

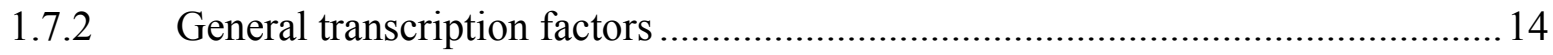

$1.8 \quad$ Exceptions to the importin $\beta$ family pathways...................................................... 15

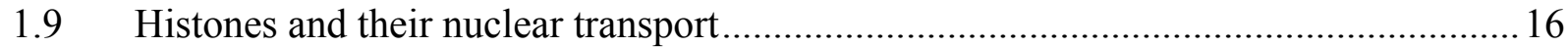

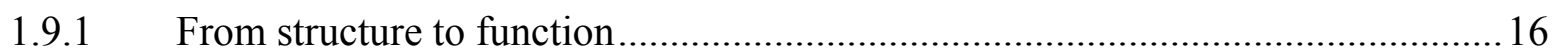

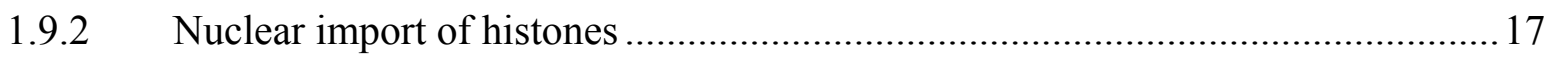

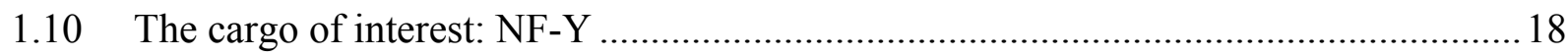

1.11 The goals of this study: What was known and what did we want to find out? ............20

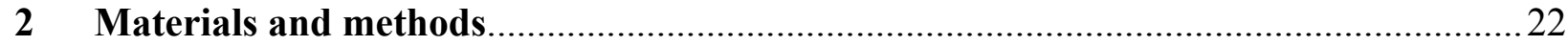

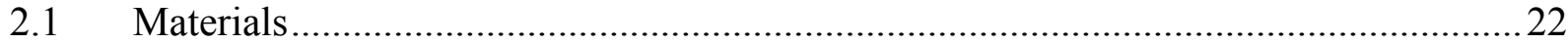

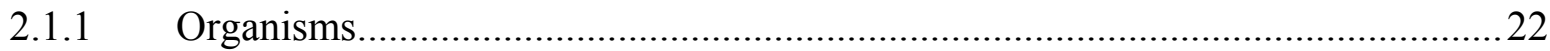

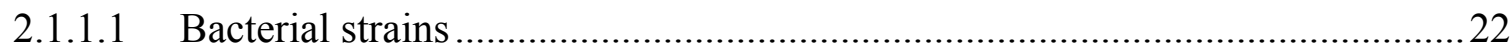

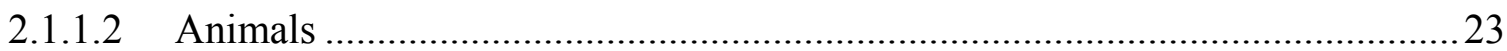

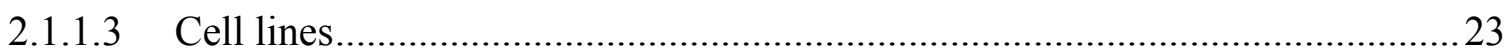

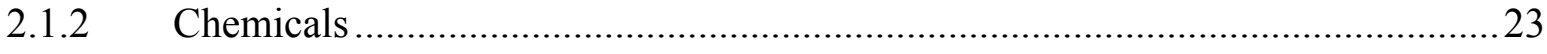

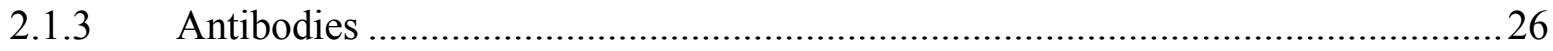

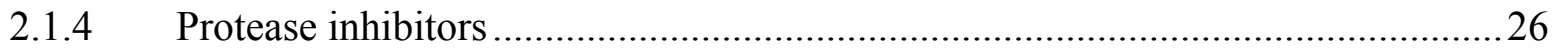

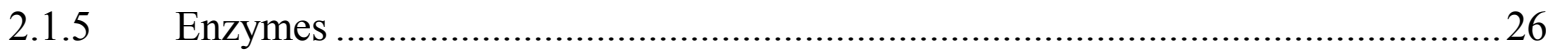

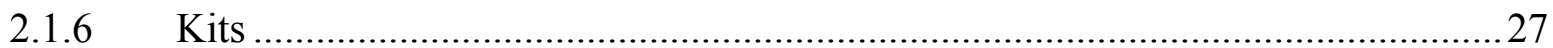




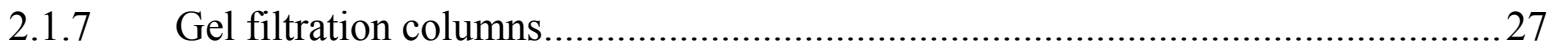

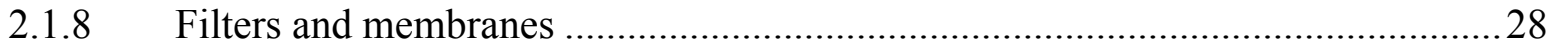

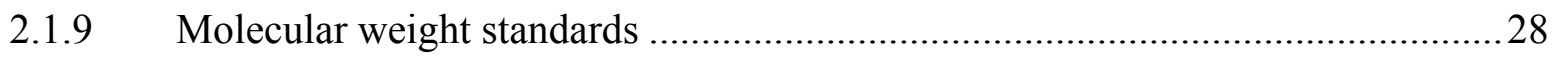

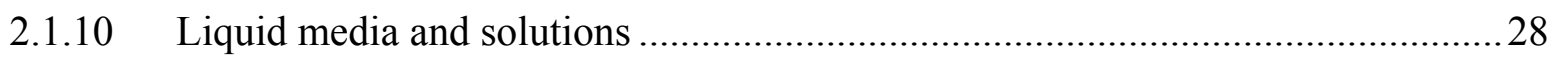

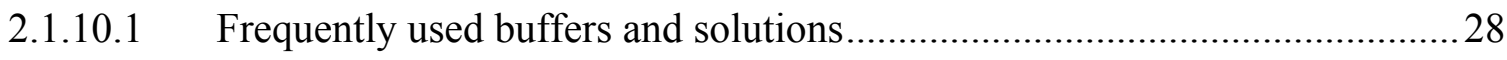

2.1.10.2 Liquid (growth) media and agar plates .....................................................29

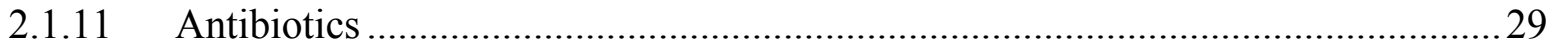

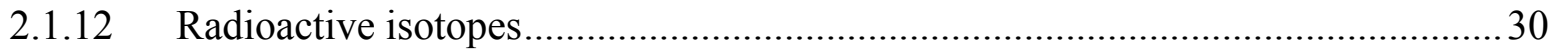

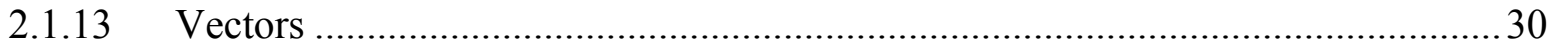

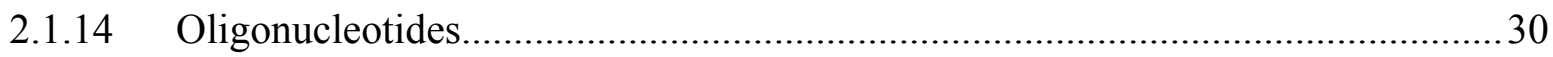

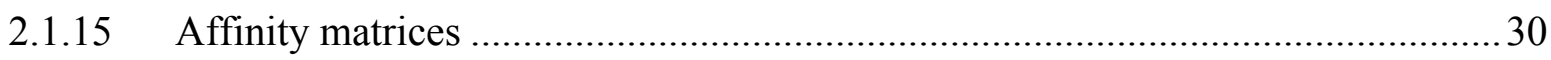

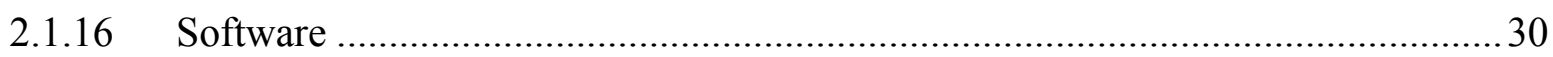

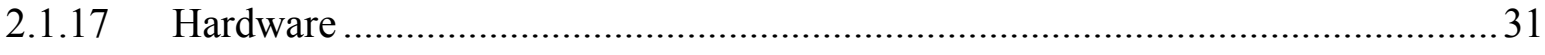

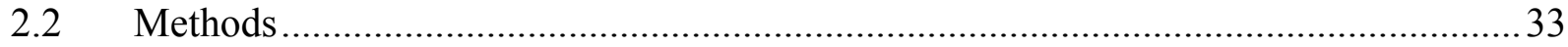

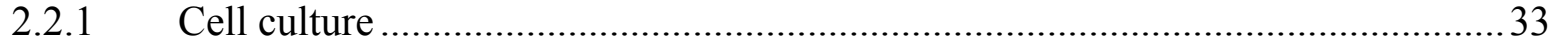

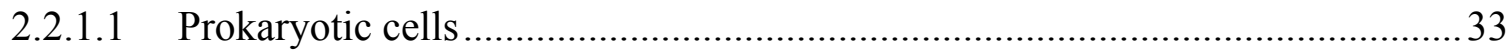

2.2.1.1.1 Bacterial cell maintenance (and growth) ............................................... 33

2.2.1.1.2 Counting bacteria: measuring the optical density ..................................... 33

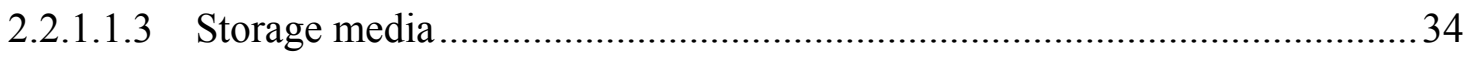

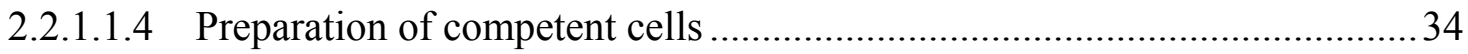

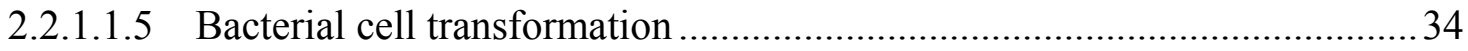

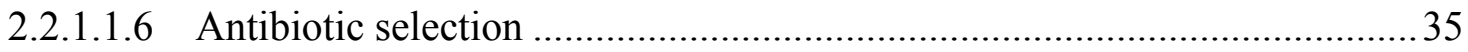

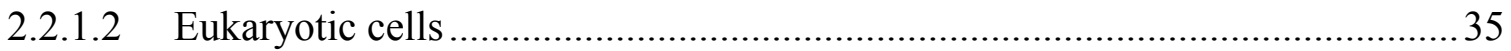

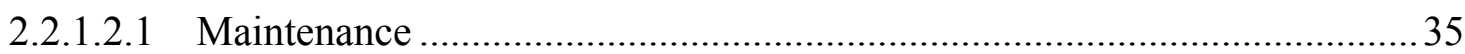

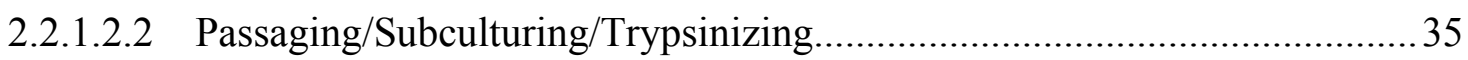

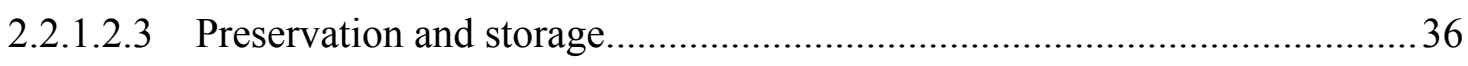

2.2.1.2.4 Transient transfection of eukaryotes .......................................................... 37

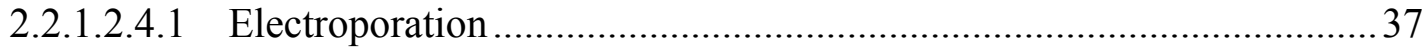

2.2.1.2.4.2 Chemical transfection (lipofection) .................................................. 37

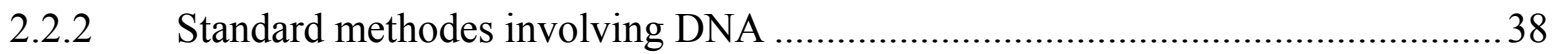

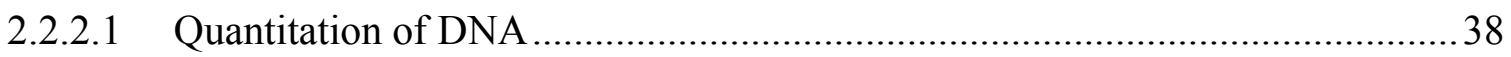

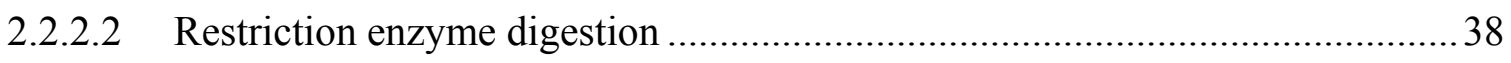

2.2.2.3 'Fill in' reactions governed by Klenow Polymerase

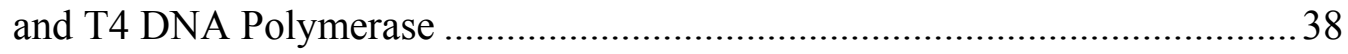




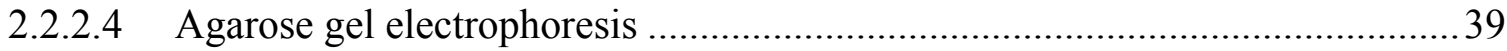

2.2.2.5 Elution of DNA fragments from agarose (QIAEX II) .................................. 40

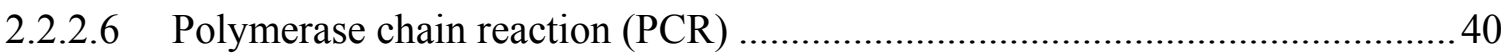

2.2.2.7 Alkaline phosphatase treatment (dephosphorylation of cut vectors) ................ 41

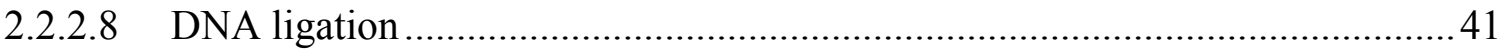

2.2.2.9 Annealing of single stranded oligonucleotides .............................................. 41

2.2.2.10 Kinase end-labeling of DNA (T4 polynucleotide kinase) .............................42

2.2.2.11 Isolation of plasmid DNA (from single colonies) after transformation ........ 42

2.2.2.12 Concentration of DNA by ethanol/isopropanol precipitation ..................... 43

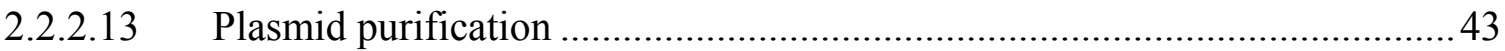

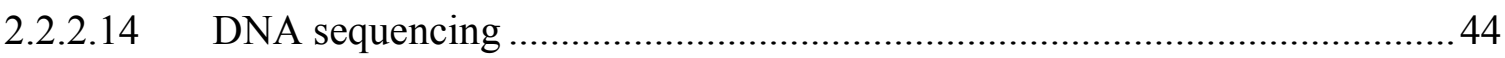

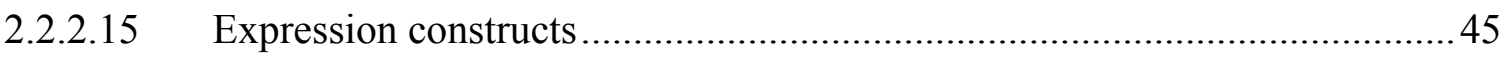

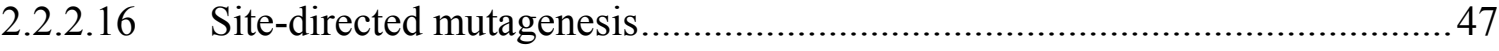

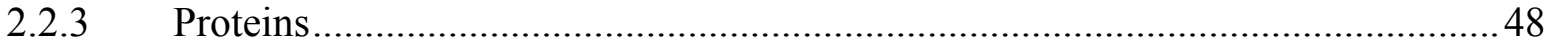

2.2.3.1 SDS-PAGE of proteins

(separation of proteins under denaturing conditions)..................................... 48

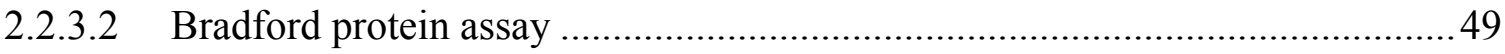

2.2.3.3 Recombinant protein expression and purification............................................50

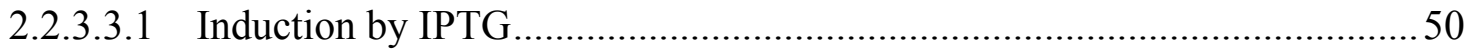

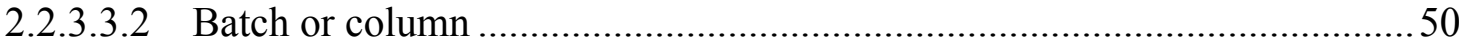

2.2.3.3.3 Affinity chromatography

(purification of proteins carrying an affinity tag)...................................50

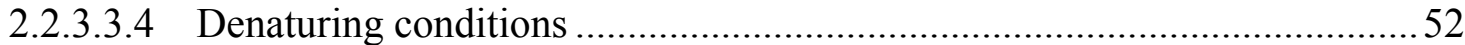

2.2.3.3.5 Expression and purification of nuclear transport cargoes ..........................53

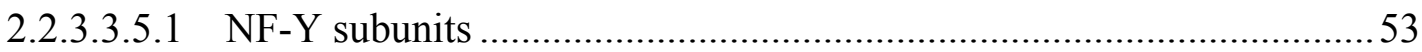

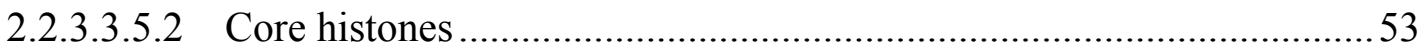

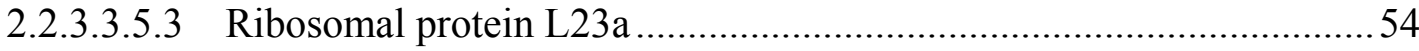

2.2.3.3.6 Expression and purification of import receptors

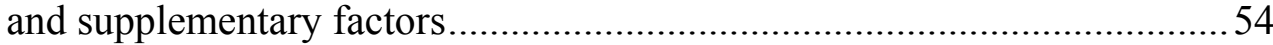

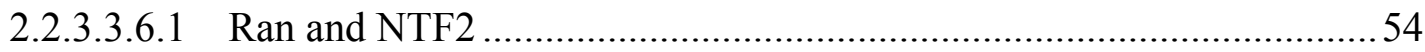

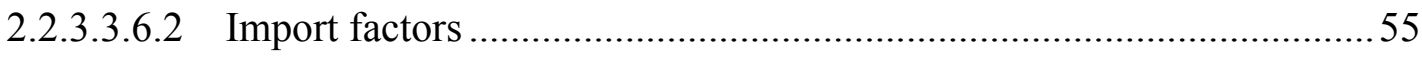

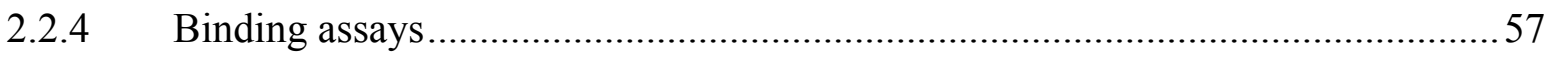

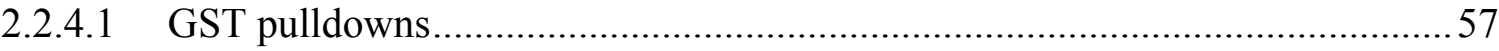

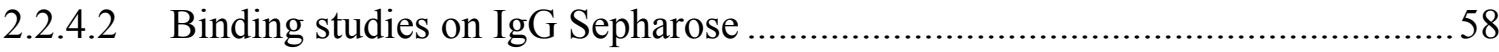


2.2.5 Preparation of fluorescence-labeled import substrates....................................58

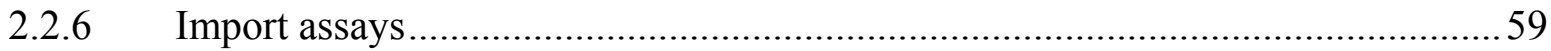

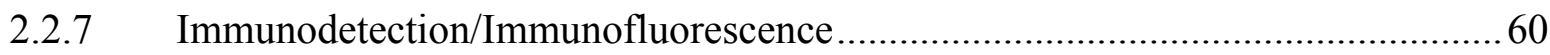

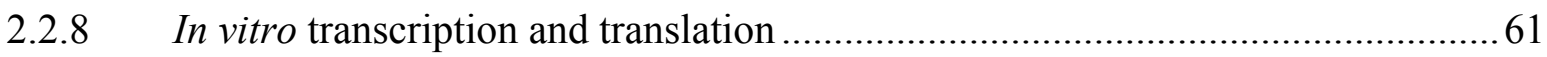

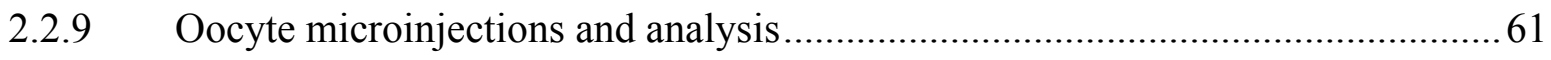

2.2.9.1 Preparation and collagenase treatment of Xenopus oocytes.............................61

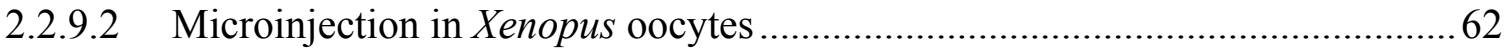

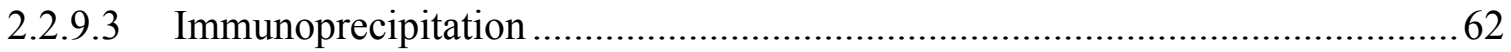

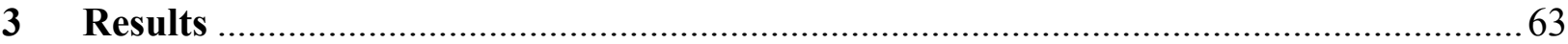

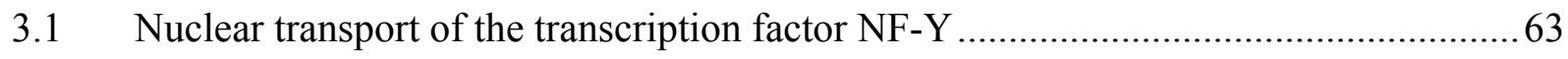

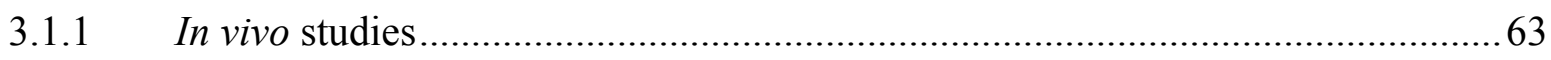

3.1.1.1 NF-Y subunits are predominantly nuclear at steady state .............................63

3.1.1.2 Explanations for the cytoplasmic localization of over-expressed NF-YC ........65

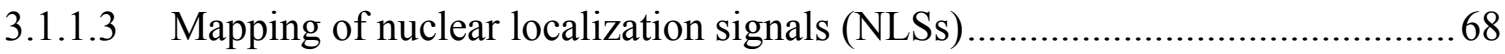

3.1.1.4 Positively charged amino acids are required

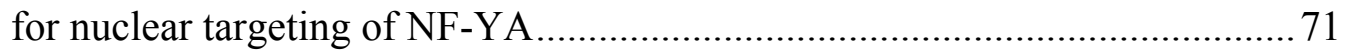

3.1.1.5 Active nuclear transport of NF-Y subunits in Xenopus oocytes ...................... 73

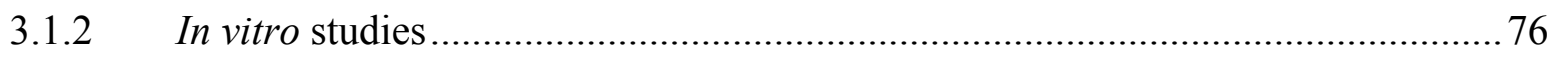

3.1.2.1 Characteristics of a receptor-mediated transport process ................................ 76

3.1.2.2 NF-Y subunits interact with different import receptors ................................... 79

3.1.2.3 Importins bind specifically to NF-Y subunits .............................................. 81

3.1.2.4 Binding sites in the NF-YB/NF-YC dimer for NF-YA and importin 13 overlap

3.1.2.5 Importin $\beta$ binding is restricted to the monomeric, uncomplexed NF-YA subunit

3.1.2.6 NF-Y subunits are imported into nuclei of permeabilized cells

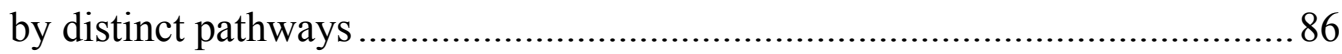

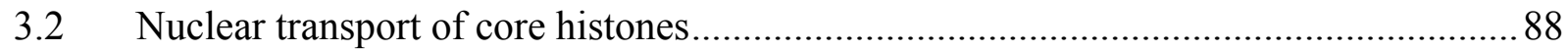

3.2.1 Importin 13 does not bind to the H2A/H2B heterodimer................................. 89

3.2.2 Interactions of import receptors with different core histone domains ................. 90

3.2.3 Amino-terminal tails of core histones contain the functional NLSs ....................99

3.2.4 Globular histone domains are not sufficient to confer nuclear import.................94

3.2.5 Nuclear transport competence of the globular domains of core histones .............96 
3.2.6 Importin $\beta$ uses the same binding site to recognize

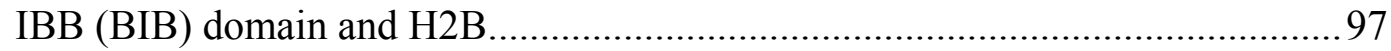

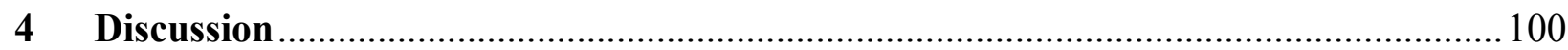

4.1 Nuclear transport of core histones: abundant cargoes, redundant pathways............... 100

4.1.1 Globular histone domains versus amino-terminal tails .................................... 102

4.1.2 Piggyback transport of globular histone domains ......................................... 103

4.1.3 Role of histone chaperones in the nuclear transport of histones ........................ 104

4.1.4 Tales about tails: amino-terminal domains as import signals ........................... 105

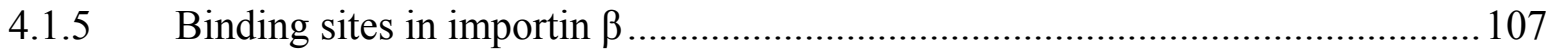

4.2 Nuclear transport of NF-Y: one complex, dual mechanism...................................... 108

4.2.1 Nuclear import of NF-YB and NF-YC depends on dimerization ...................... 109

4.2.2 Nuclear import of NF-YA: monomeric, but regulated.................................... 112

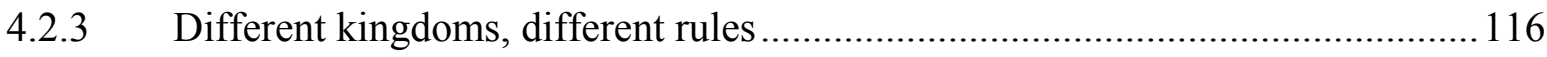

4.2.4 NF-YB and NF-YC fulfill nuclear functions independent of NF-YA ............... 117

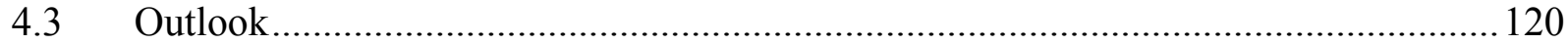

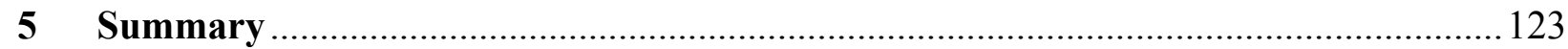

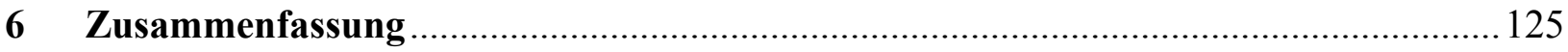

$7 \quad$ References

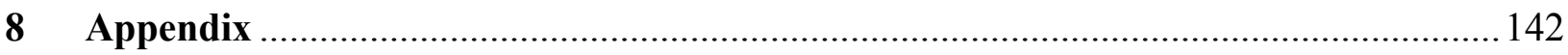




\section{Figure index}

FIG. 1. The nuclear pore complex (NPC) - tunnel between nucleus and cytoplasm.

FIG. 2. Schematic representation of the nuclear import and export pathway coordinated by the RanGTPase system.

FIG. 3. NF-Y subunits and their association.

FIG. 4. Subcellular localization of NF-Y subunits in HeLa cells.

FIG. 5. Nuclear export does not cause the cytoplasmic

localization of over-expressed NF-YC.

FIG. 6. Nuclear transport of NF-YB and NF-YC fragments fused to ß-galactosidase.

FIG. 7. NF-YA contains a NLS in the evolutionarily conserved domain.

FIG. 8. Characterization of the ncNLS of NF-YA by mutagenesis of basic clusters; positive charge is required for nuclear targeting.

FIG. 9. Active nuclear transport of NF-Y subunits in Xenopus laevis oocytes. 75

FIG. 10. Nuclear import of NF-YA shows characteristics of a receptor-mediated process while monomeric HFM containing subunits are not transported into the nuclei of permeabilized cells. 78

FIG. 11. Interaction of NF-Y subunits with different import receptors. 80

FIG. 12. Specific binding of importins to NF-Y subunits. 82

FIG. 13. Binding sites in the NF-YB/NF-YC complex for NF-YA and importin 13 overlap.

FIG. 14. NLS in NF-YA can not be further recognized by importins

once the subunit is assembled into the NF-Y complex.

FIG. 15. NF-Y subunits are imported into nuclei of permeabilized cells

by distinct pathways. .87

FIG. 16. Importin 13 does not bind to the $\mathrm{H} 2 \mathrm{~A} / \mathrm{H} 2 \mathrm{~B}$ heterodimer. 90

FIG. 17. Interactions of import receptors with different core histone domains. . 91

FIG. 18. Importins bind specifically to the amino-terminal tails of $\mathrm{H} 2 \mathrm{~A}$ and $\mathrm{H} 2 \mathrm{~B}$. .93

FIG. 19. Importins bind specifically to the amino-terminal tails of $\mathrm{H} 3$ and $\mathrm{H} 4$. 94

FIG. 20. Globular domains of the core histones H2A, H2B, H3, and H4 are not sufficient to confer nuclear accumulation in the nuclei of permeabilized cells. 95

FIG. 21. Piggyback transport of the globular domain of H2B. 97

FIG. 22. Core histones presumably bind to the cargo binding site I on importin $\beta$. 98

FIG. 23. Sequence alignment of core histones in lower and higher eukaryotes. 105 


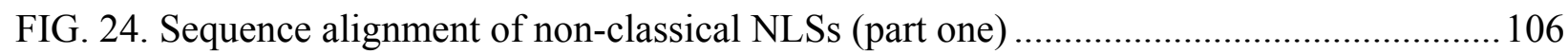

FIG. 25. Sequence alignment of non-classical NLSs (part two) ............................................ 113

FIG. 26. Proposed model for the nuclear transport of NF-Y subunits in higher eukaryotes. ..... 115

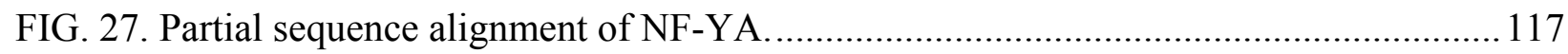




\section{Abbreviations}

\begin{tabular}{|c|c|}
\hline $\mathbf{a a}$ & amino acid \\
\hline ad & add up to \\
\hline amp & ampicilline \\
\hline APS & ammonium peroxosulfate \\
\hline ARM & armadillo \\
\hline ATP & adenosine triphosphate \\
\hline BIB & beta-like import receptor binding \\
\hline BME & $\beta$-mercaptoethanol \\
\hline $\mathrm{bp}$ & base pairs \\
\hline BSA & bovine serum albumin \\
\hline CRM & chromosome region maintenance \\
\hline d & distilled \\
\hline $\mathrm{Da}$ & Dalton \\
\hline DAPI & 4'-6-Diamino-2-phenylindol-dihydrochlorid \\
\hline ddNTP & dideoxy nucleoside triphosphate (ddATP, ddCTP, ddGTP, ddTTP) \\
\hline DMSO & dimethyl sulfoxide \\
\hline DNA & deoxyribonucleic acid \\
\hline dNTP & deoxyribonucleoside triphosphate (dATP, dCTP, dGTP, dTTP) \\
\hline ds & double strand \\
\hline DTT & dithiothreitol \\
\hline E. coli & Escherichia coli \\
\hline EDTA & ethylenediaminetetraacetate \\
\hline EGTA & ethylene-bis(oxyethylenenitrilo)tetraacetic acid \\
\hline eIF-5A & eukaryotic translation initiation factor $5 \mathrm{~A}$ \\
\hline EST & expressed sequence tag \\
\hline FCS & fetal calf serum \\
\hline Fig. & figure \\
\hline FITC & fluorescein isothiocyanate \\
\hline FRET & fluorescence resonance energy transfer \\
\hline GDP & guanosine-diphoshate \\
\hline GFP & green fluorescent protein \\
\hline GST & glutathione S-transferase \\
\hline
\end{tabular}


GTP guanosine triphosphate

HA hemagglutinin

HEAT ( huntingtin, elongation factor 3, "A $\underline{\text { " }}$ subunit of protein phosphatase A, and TOR1 lipid kinase)

HEPES N-2-hydroxyethylpiperazine- $N$ '-2-ethanesulfonic acid

IBB importin $\beta$ binding

IgG immunoglobulin $\mathrm{G}$

Kap karyopherin

$\mathrm{kDa} \quad$ kilodalton

LB Luria Bertani

LMB leptomycin B

MCS multiple cloning site

MGN Mago Nashi protein homologue

mRNA messenger RNA

NE nuclear envelope

NES nuclear export signal

NF-Y nuclear factor $\mathrm{Y}$

NLS nuclear localization signal

NPC nuclear pore complex

NTF2 nuclear transport factor 2

OD optical density

p plasmid

p.a. pro analysi

PAGE polyacrylamide gelelectrophoresis

PBS phosphate buffered saline

PCR polymerase chain reaction

PKI protein kinase inhibitor

PTHrP parathyroid hormone-related protein

Ran Ras related nuclear protein

RanBP Ran binding protein

RanGAP Ran GTPase activating protein

RanGEF Ran guanine nucleotide exchange factor

RCC1 regulator of chromosome condensation 1

RNA ribonucleic acid 


\begin{tabular}{ll} 
RNase & ribonuclease \\
RNP & ribonucleoprotein \\
rRNA & ribosomal RNA \\
SDS & sodium dodecyl sulfate \\
snRNP & small nuclear RNP \\
SUMO & small ubiquitin-related modifier/modification \\
SV40 & simian virus 40 \\
TAF & TBP-associated factor \\
TBP & TATA-binding protein \\
TBS & Tris buffered saline \\
TE & Tris-EDTA-buffer \\
TELT & Tris-EDTA-LiCl-Triton \\
TEMED & tetramethylethylenediamine \\
TFIIA & transcription factor II A \\
TFIID & transcription factor II D \\
TRIS & tris(hydroxymethyl)aminomethane \\
TRITC & tetramethylrhodamine isothiocyanate \\
tRNA & transfer RNA \\
UV & ultraviolet \\
WGA & wheat germ agglutinin \\
wt & wild type \\
\hline
\end{tabular}

$\underline{\text { One letter code for amino acids: }}$

$\begin{array}{llll}\text { A } & \text { alanine } & \mathrm{M} & \text { methionine } \\ \mathrm{C} & \text { cysteine } & \mathrm{N} & \text { asparagine } \\ \mathrm{D} & \text { aspartic acid } & \mathrm{P} & \text { proline } \\ \mathrm{E} & \text { glutamic acid } & \mathrm{Q} & \text { glutamine } \\ \mathrm{F} & \text { phenylalanine } & \mathrm{R} & \text { arginine } \\ \mathrm{G} & \text { glycine } & \mathrm{S} & \text { serine } \\ \mathrm{H} & \text { histidine } & \mathrm{T} & \text { threonine } \\ \mathrm{I} & \text { isoleucine } & \mathrm{V} & \text { valine } \\ \mathrm{K} & \text { lysine } & \mathrm{W} & \text { tryptophan } \\ \mathrm{L} & \text { leucine } & \mathrm{Y} & \text { tyrosine }\end{array}$




\section{Introduction}

\subsection{The past: recent history}

As in everyday life also within the smallest unit of life, the cells, certain barriers sometimes can not be overcome without the help of others. In eukaryotic cells one of these barriers consists of the nuclear envelope that separates the nucleus from the cytoplasm and restricts nucleocytoplasmic exchange of material to nuclear pore complexes (NPCs) which are embedded in the nuclear membranes. For a long time, one of the big questions was the identity of the system escorting the variety of macromolecular cargoes between the nucleus and cytoplasm. Since more than a decade now, it is known that nuclear transport receptors mediate most of the transport events through the NPC. The in vitro system that allowed to study biochemical transport events across the nuclear envelope was developed almost 15 years ago by Adam et al. (Adam et al., 1990). Cell treatment with digitonin selectively permeabilizes the plasma membranes but the nuclear envelope remains structurally intact, and nuclei retain the ability to transport and to accumulate proteins containing a nuclear localization signal (NLS). With this powerful tool for investigating biochemical pathways of nuclear transport in hands, Moore and Blobel (Moore and Blobel, 1992) reported about the isolation of two cytosolic fractions (A and B) from Xenopus ovary cells that were sequentially required to support nuclear protein import. Fraction A was required for recognition of the NLS and targeting to the nuclear envelope, whereas fraction $\mathrm{B}$ mediated the subsequent translocation of the bound substrate into the nucleus. The two interacting components required for fraction B activity were identified as Ran (Ras-related nuclear protein)/TC4 (Moore and Blobel, 1993) and NTF2 (nuclear transport factor2)/p10 (Moore and Blobel, 1994; Paschal and Gerace, 1995). The missing component of fraction A, essential for the first step of selective protein import into the nucleus, was then finally identified by Gorlich et al. (Gorlich et al., 1994). Due to its function the protein was named importin. Soon it became obvious that importin actually consisted of two independent import receptors, namely importin $\alpha$ and importin $\beta$, the latter had been dissociated from importin $(\alpha)$ during the initial purification. Soon, distinct functions for these two importins in nuclear protein import were found. Thus, importin $\alpha$ is primarily responsible for NLS recognition, whereas targeting of the importin-cargo complex to the NPC and subsequent docking are achieved by importin $\beta$ (Gorlich et al., 1995). Nowadays, we know that 20 importin $\beta$ family members exist in human cells. These interact with cargo molecules independent of importin $\alpha$. A total of 10 family members are known to function in import, seven in export and one in both directions, and a major effort in the field of nucleocytoplasmic transport is directed towards the identification of cargoes for the individual importin $\beta$ family members. 


\subsection{The field: nucleocytoplasmic transport}

The existence of a nucleus in eukaryotic cells implies the spatial separation of transcription (nucleus) and translation (cytoplasm). This provides eukaryotic cells with additional means to regulate the fundamental process of gene expression. The confinement of transcription and translation to distinct compartments also prevents translation before splicing has been completed. The splicing process is necessary because most eukaryotic genes contain introns. However, nuclei contain many of the components required for translation such as ribosomal subunits, mRNA and even aminoacylated tRNA (Lund and Dahlberg, 1998), whereas proteins whose function is clearly nuclear (e.g. histones, transcription factors, DNA polymerases) are synthesized in the cytoplasm. Thus, bidirectional intracellular trafficking of macromolecules between these two compartments is required and consumes a considerable amount of the cell's energy pool. Thereby, selective transport of proteins is a major mechanism by which biochemical differences are maintained between the cytoplasm and nucleus. The site of exchange is the nuclear pore complex (NPC), one of the largest macromolecular assemblies in a eukaryotic cell. It is embedded in the double membrane of the nuclear envelope. The NPC connects the cytoplasm and the nucleus of interphase cells and can be traversed in a passive or a facilitated manner. The passive mode applies for ions, ATP and small molecules but becomes ineffective for proteins with a molecular weight greater than 40-60 $\mathrm{kDa}$. A single NPC can accommodate about 1000 translocation events per second (reviewed in Fried and Kutay, 2003) and the cargoes range in mass from few $\mathrm{kDa}$ to almost $50 \mathrm{MDa}$ that equals almost $40 \mathrm{~nm}$ in diameter (Pante and Kann, 2002; Rout and Aitchison, 2001). Unlike translocation across other organelle membranes, passage through the NPC does not require unfolding of the substrates; however, the mechanistic details of the translocation event and the principles of NPC function have not been completely unveiled. In addition, also molecules which potentially may passively diffuse through the NPC are often actively translocated, since this allows a more efficient and regulated transport (for reviews, see references Conti and Izaurralde, 2001; Gorlich and Kutay, 1999; Mattaj and Englmeier, 1998; Rout and Aitchison, 2001; Weis, 2003). In general, four major categories of active nucleocytoplasmic transport can be distinguished. First, biosynthetic transport, as mentioned above, supplying the two different compartments with macromolecules that have been produced in the opposite one (reviewed in Gorlich and Kutay, 1999; Mattaj and Conti, 1999). The second category includes the so-called recycling reactions in which cargo-free transport receptors or transport adaptors are returned back to the compartment, where the next cargo molecule should be loaded (Kutay et al., 1997a). Third, regulated traffic of cargoes between nucleus and cytoplasm, which is used to control key cellular processes (Kaffman and 
O'Shea, 1999; Schuller and Ruis, 2002). For instance, the activity of many transcription factors is controlled at least partially by their subcellular localization (see also chapter 1.7). Finally, also the nuclear export machinery can be considered as an independent category of active nucleocytoplasmic transport. Exportin-mediated transport counteracts the slow but steady leakage of cytoplasmic proteins into the nucleus (Bohnsack et al., 2002; Mingot et al., 2004; Stuven et al., 2003) and removes proteins that have unintentionally entered the nucleus (for review, see Fornerod and Ohno, 2002).

\subsection{The site of action: nuclear pore complex}

The nuclear pore complex (NPC) is one of the largest protein assemblies in eukaryotic cells, ranging in mass between $50 \mathrm{MDa}$ in yeast and $125 \mathrm{MDa}$ in vertebrates (Fahrenkrog et al., 2001; Rout and Aitchison, 2001). Three-dimensional structures of the NPC have been derived from electron microscopy studies in yeast (Yang et al., 1998) and in higher eukaryotes (Xenopus oocytes) (Akey and Radermacher, 1993; Hinshaw et al., 1992; Stoffler et al., 2003) indicating that the core of the NPC consists of a cylinder with eight-fold symmetry (called spoke complex) that spans the nuclear envelope. Cytoplasmic and nuclear ring moiety sandwich the central framework and peripheral filaments originate from each ring moiety, pointing towards the cytoplasm and nucleoplasm, respectively. The eight nuclear filaments are held together in a distal ring forming a distinct assembly called nuclear basket. The central pore (channel) created by the spoke complex has a length of about $90 \mathrm{~nm}$ and a narrowest diameter of 45-50 nm (Fahrenkrog and Aebi, 2003; Fahrenkrog et al., 2004; Stoffler et al., 2003). Pante and Kann, 2002 found that cargo-receptor-gold complexes with a diameter up to $40 \mathrm{~nm}$ could be translocated by the NPC indicating that its physical diameter coincides with its functional diameter (Pante and Kann, 2002). With regard to the molecular architecture, in yeast and in mammals the NPC is composed of only 30 different proteins called nucleoporins (Cronshaw et al., 2002; Rout et al., 2000). The massive NPC can be constructed from few distinct proteins because of the high copy number of the individual nucleoporins, owing to the eight-fold rotational symmetry of the NPC. Approximately one third of all core nucleoporins contains domains of phenylalanine-glycine (FG) repeats (Cronshaw et al., 2002; Rout et al., 2000), which mediate the main interaction between transport factors and nucleoporins.

On the basis of the affinity and the nature of interaction, different models of facilitated translocation through the NPC have been proposed (Macara, 2001; Ribbeck and Gorlich, 2001; Rout et al., 2000). The Brownian affinity gate model (Rout et al., 2000) proposes that the aqueous NPC channel consists of a narrow central tube. Binding to peripheral FG-repeats 


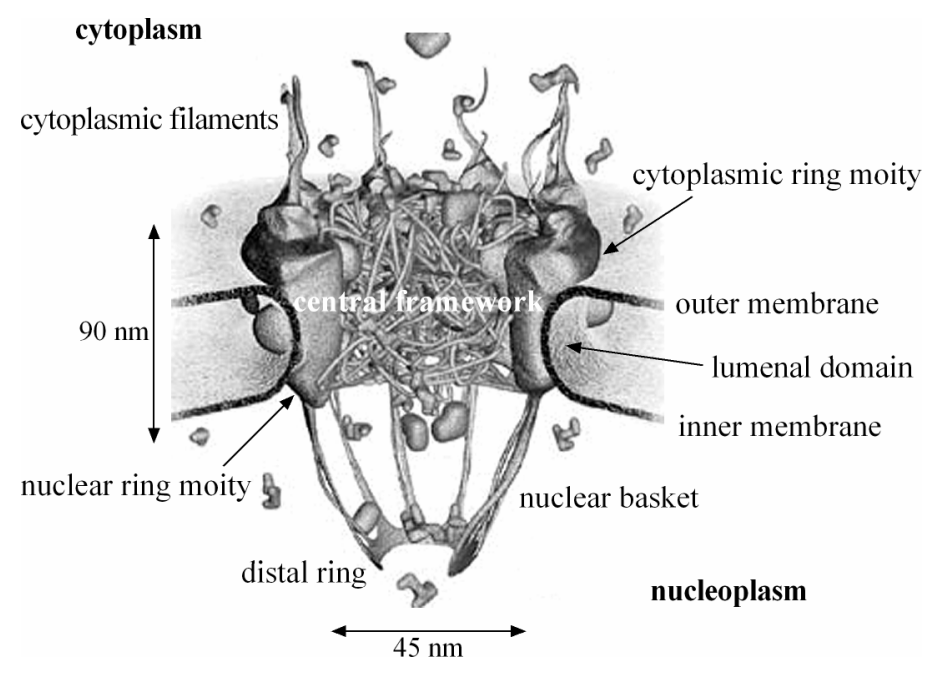

FIG. 1. The nuclear pore complex (NPC) - tunnel between nucleus and cytoplasm. A model of the 3D architecture of the NPC based on electron microscopy studies (for more detail, see text). The major structural components of the NPC include the central framework with eight-fold rotational symmetry (built around a central pore), the cytoplasmic ring and filaments, and the nuclear ring and basket. Unstructured FG-rich repeat filaments are proposed to fill the central pore channel which represents the exclusive gateway for nucleocytoplasmic transport pathways. In the selective phase model (Ribbeck and Gorlich, 2001; Ribbeck and Gorlich, 2002) weakly interacting, hydrophobic FG-rich repeats form a meshwork which can only be entered and permeated by transport receptors. The cytoplasmic fibrils and the nuclear basket are involved in the initial and terminal stages of translocation (this figure was adapted from a NPC picture created by Samir S. Patel; http://www.stanford.edu/group/rexachlab/research).

facilitates the entry into the pore and then allows translocation by Brownian motion. The selective phase model is based on kinetic data presented by Ribbeck and Gorlich (Ribbeck and Gorlich, 2001; Ribbeck and Gorlich, 2002). As the name indicates, this model proposes that the central channel of the NPC is filled with a selective phase consisting of a meshwork formed by weakly interacting, hydrophobic FG repeats. This meshwork functions as kind of a sieve; presenting a significant permeability barrier for inert molecules, but becoming selectively permeable for transport receptors that can interact with the FG-repeats and disrupt the meshwork. Very similar to the selective phase model is the 'oily-spaghetti' model suggesting that the NPC channel is filled by hydrophobic, unstructured, non-interacting FG-repeats (Macara, 2001). These 'oily-spaghetti' can be pushed aside by transport receptors (loaded with a cargo or unloaded) whereas they prevent the translocation of other molecules.

In conclusion; all three models have two things in common: Firstly, the NPC selectivity is exclusively achieved by the conserved FG-repeats. Secondly, transport trough the pores occurs via diffusion. 


\subsection{The mediators: importins and exportins}

Most nuclear transport processes are mediated by soluble transport receptors that recognize specific sequences or structural characteristics of their cargoes and facilitate the passage of receptor-cargo complexes through the NPC. Transport receptors constantly shuttle between the nucleus and cytoplasm thereby rapidly crossing the permeability barrier of nuclear pores (Ribbeck and Gorlich, 2001). The biggest class of nuclear transport receptors is the superfamily of importin $\beta$ like factors (also named karyopherins) that can be classified as importins (import karyopherin) and exportins (export karyopherin) depending on the direction in which they transport the cargo (reviewed in Gorlich and Kutay, 1999; Imamoto, 2000; Macara, 2001; Strom and Weis, 2001; Wozniak et al., 1998). Common features of the importin $\beta$ family members are an amino-terminal RanGTP-binding domain, a similar large size (90-130 kDa), and an acidic isoelectric point (4.6-5.9) (Gorlich et al., 1997). 20 members of this importin $\beta$ family were identified in mammals (see also appendix, table I). For ten of these (importin $\beta$, importin 4 , importin 5, importin 7, importin 8, importin 9, importin 11, transportin, transportin 2, transportin $\mathrm{SR})$, a role in nuclear import was acknowledged, seven were shown to function in nuclear export (Crm1/exportin1, CAS, exportin-t, exportin 4, exportin 5, exportin 6, exportin 7), and one can mediate transport in both directions (importin 13), leaving two uncharacterized (RanBP6, RanBP17). Considering the low number of transport receptors and the large number of proteins that have to be translocated, most transport factors probably recognize many cargoes. Just the other way around, the identification of multiple import receptors for certain cargoes (such as ribosomal proteins and histones; see also chapter 1.9.2) revealed a significant redundancy between transport pathways (for a review and a cargo list, see Strom and Weis, 2001). An intriguing question regarding this subject is, how does a given import receptor recognize many structurally different proteins?

Structural studies have focused so far mainly on importin $\beta$ revealing that the structure of this import receptor consists of 19 tandem HEAT repeats forming a superhelical structure (snaillike). HEAT repeats are named after the four proteins (huntingtin, elongation factor 3, ' $\underline{A}$ ' subunit of protein phosphatase A, and TOR1 lipid kinase) in which the motif was identified first (Andrade and Bork, 1995). Each HEAT repeat consists of two helices (A and B) connected by a loop. The A helices are located on the outside of the protein and form the convex face, whereas the B helices are located at the inside forming the concave surface (Cingolani et al., 1999). Interactions with the NPC (FG repeats) occur on the outer surface of the helical structure (Bayliss et al., 2000), whereas cargoes such as the IBB domain (HEAT 7-19) (Cingolani et al., 1999), PTHrP (HEAT 1-11) (Cingolani et al., 2002), and RanGTP (HEAT 1-10) (Vetter et al., 
1999) bind the indicated regions of the inner concave surface. Furthermore, structural differences were observed between RanGTP bound and unbound importin $\beta$, suggesting that importin $\beta$ undergoes large conformational changes in the HEAT repeat arrangement upon RanGTP binding (Lee et al., 2000). Taken together, structural analysis strongly indicates that the spring-like superhelical conformation of importin $\beta$ has a large degree of flexibility (Stewart, 2003). This inherited flexibility provides a rational explanation for the existence of many distinct substrate binding sites in importin $\beta$. Recently, two independent studies have illuminated the structural basis and the molecular mechanisms for the assembly of nuclear export complexes (Matsuura and Stewart, 2004; Petosa et al., 2004). Both studies revealed that exportins (such as CAS and Crm1) are also constructed from superhelices of HEAT repeats suggesting that presumably all members of the importin $\beta$ family share this structural feature. Further, it was shown that a conformational change in the Ran binding site (loop) of Crm1 (exportin 1) accounts for the cooperativity of Ran- and cargo binding (Petosa et al., 2004) and that a second (C-terminal) Ran binding site as identified in CAS (exportin 2) may be a general feature of export receptors (Matsuura and Stewart, 2004).

In contrast to the structure of the importin $\beta$ family members, importin $\alpha$ does not consist of HEAT repeats but consists of ten tandemly arranged, armadillo (ARM) repeats forming a cylindrical superhelix (Conti et al., 1998; Kobe, 1999). An individual ARM repeat contains approximately 40 amino acids that form three helices. Superposition of individual importin $\alpha$ ARM repeats with importin $\beta$ HEAT repeats indicates significant structural similarities, suggesting that these two structures belong to the same family of helical repeat motifs (Cingolani et al., 1999; Malik et al., 1997; Vetter et al., 1999). Besides the ARM repeat domain which harbors the NLS-binding site, importin $\alpha$ contains a short carboxyl terminus and a basic, autoinhibitory, amino-terminal importin $\beta$ binding (IBB) domain. The IBB domain can bind either to ARM repeats (auto-inhibition; Kobe, 1999) or to importin $\beta$ forming the functional importin $\alpha / \beta$ heterodimer (Gorlich et al., 1996a). In this case, interaction of importin $\beta$ with the IBB domain is analogous to a direct importin $\beta$-cargo interaction in the absence of the importin $\alpha$ adaptor. Binding of positively charged amino acid sequences (basic NLSs) is in both cases a result of hydrophobic interactions combined with electrostatic interactions on the surface of the molecule. However, despite the structural similarities regarding the repeating units (HEAT and ARM) and despite using similar binding strategies, importin $\alpha$ and importin $\beta$ have different NLS sequence requirements for their cargoes. In general, classical positively charged NLSs that bind to importin $\alpha$ are too short to be recognized by importin $\beta$ (for reviews, see Chook and Blobel, 2001; Conti, 2002; Conti and Izaurralde, 2001). 


\subsection{The key regulator: Ran}

Cargo binding and release of importins and exportins is controlled by a steep RanGTP gradient which is maintained across the nuclear envelope through the asymmetric distribution of factors that regulate the guanine nucleotide-bound state of Ran (Gorlich and Kutay, 1999; Lei and Silver, 2002; Macara, 2001; Mattaj and Englmeier, 1998; Weis, 2002). The guanine exchange factor, RanGEF (also called RCC1), is exclusively nuclear, while the GTPase-activating protein (RanGAP) together with the accessory proteins RanBP1 and RanBP2 are cytoplasmic. RanGAP is found at its highest concentration at the cytoplasmic periphery of the NPC, where it associates with the nucleoporin RanBP2 via its SUMO modification (Mahajan et al., 1997; Matunis et al., 1996). Recent fluorescence resonance energy transfer (FRET)-based biosensor data confirmed the presence of a RanGTP gradient in interphase nuclei (cells) and the concentration difference between nuclear and cytoplasmic RanGTP was estimated to be at least 200-fold (Kalab et al., 2002).

Importins and exportins interact with RanGTP through their conserved amino-terminal domains, but binding of RanGTP has the opposite effect on these receptors. Importins load cargoes in the absence of Ran in the cytoplasm and release their cargo upon RanGTP binding in the nucleus (Gorlich et al., 1996b; Izaurralde et al., 1997; Rexach and Blobel, 1995). In contrast, exportins bind substrates only in the presence of RanGTP in the nucleus and cargo release is accomplished when the Ran-bound GTP molecule is hydrolyzed in the cytoplasm (Bischoff and Gorlich, 1997; Fornerod et al., 1997a; Kutay et al., 1997a). For a single round of nuclear import GTP hydrolysis is not needed because translocation through the NPC itself is a fully reversible process (Englmeier et al., 1999; Schwoebel et al., 1998). However, directional transport is only achieved by Ran which regulates cargo binding and release of the transport receptors. Thus, GTP hydrolysis constitutes the sole input of metabolic energy, in form of at least one molecule of GTP hydrolyzed per transport cycle, which allows import and export cargoes to accumulate against gradients of chemical activities (Gorlich and Kutay, 1999; Gorlich et al., 2003; Kose et al., 1997; Ribbeck et al., 1999; Weis et al., 1996a).

When transport receptors directly bind to their cargo, a single round of RanGTP hydrolysis is expended to translocate one cargo uni-directionally through the NPC. However, in case of transport receptors which carry cargoes bi-directionally across the nuclear envelope, such as importin 13 in mammals (Mingot et al., 2001) and Msn5 in yeast (Yoshida and Blobel, 2001), transport of two substrates in opposite directions per RanGTP hydrolysis cycle is achieved. This obvious increase in efficiency because of lesser energy expenditure, however, drastically limits the endpoint of cargo accumulation. The export of one RanGTP molecule down the RanGTP 
gradient must drive the transport of two cargoes against their gradients of chemical activity (Mingot et al., 2001). As a result, bi-directional transport by a single carrier is probably an exception rather than the rule. In addition, some importins as importin $\beta$ also use adaptor proteins to interact with their cargo. Classical NLS (cNLS) containing proteins are recognized by the importin $\alpha / \beta$ heterodimer, where importin $\alpha$ binds to the cNLS and importin $\beta$ is required for translocation through the NPC. Since both importins return independently to the cytoplasm in a RanGTP-complex (importin $\beta /$ RanGTP and importin $\alpha /$ CAS/RanGTP) (reviewed in Gorlich and Kutay, 1999; Mattaj and Englmeier, 1998) two RanGTP molecules are expended for this classical NLS import cycle.

No matter in which way RanGTP depletion from the nucleus occurs, it has to be continuously refilled. RanGDP could theoretically diffuse into the nucleus; however, it was shown that its nuclear uptake is facilitated by the Ran import receptor NTF2/p10 (Ribbeck et al., 1998; Smith et al., 1998) which particularly recognizes the GDP-conformation of Ran (Stewart et al., 1998a). In the nucleus, RanGDP is recharged with GTP by the action of nucleotide exchange factor RanGEF (Bischoff et al., 2002) which also leads to the dissociation of RanGTP from NTF2. In conclusion, Ran has to cross the nuclear envelope as frequently as all members of the importin $\beta$ family together and therefore probably represents the most rapidly shuttling macromolecule in the cell.

\subsection{The signals: NLS and NES}

Receptor-mediated, active nuclear transport of proteins between the nucleus and cytoplasm depends on localization signals in the cargoes. Depending on the direction in which the macromolecules are transported, the signals can be classified in nuclear localization signals (NLSs) and nuclear export signals (NESs). In general, these signals are defined by amino acid regions (peptide sequences) of the respective cargoes but the size and structure of transport signals vary considerably. In contrast to other peptide signals or targeting sequences (as for instance ER targeting signals), NLS and NES are not cleaved during/after transport because they (i) are not located at either the $\mathrm{N}$ - or C-terminal end, and (ii) are often required to function many times depending on the cargo's duration of life (e.g. shuttling proteins and transcription factors). However, the most dramatic event in the cells of higher eukaryotes is mitosis. Once per cell cycle/division the nuclear envelope breaks down, the content of the nuclear and cytoplasmic compartments becomes completely mixed, and eventually has to be sorted out. This of course requires intrinsic protein sorting signals. 


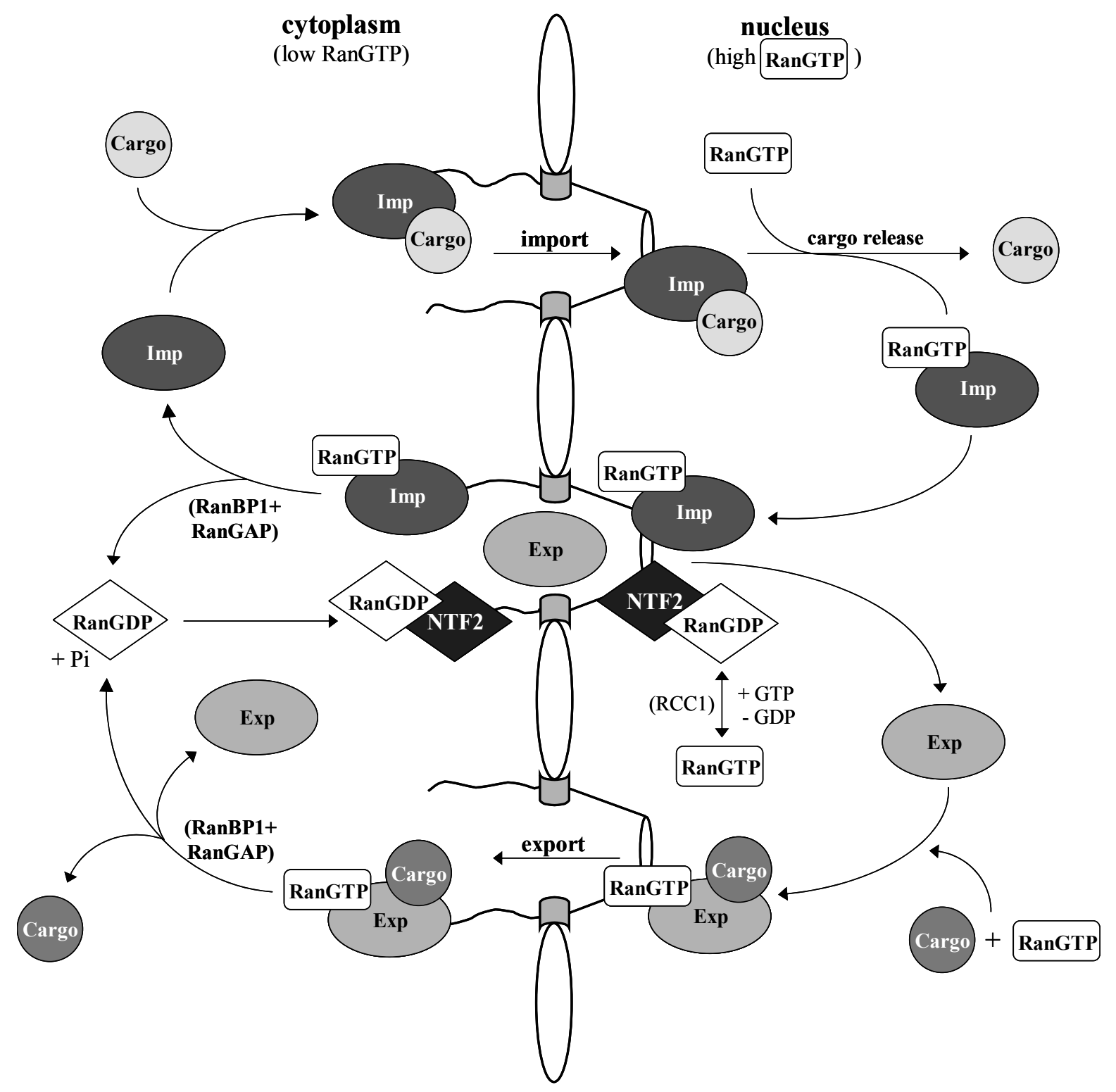

FIG. 2. Schematic representation of the nuclear import and export pathway coordinated by the

RanGTPase system. Cargo binding and release of importins (Imp) and exportins (Exp) is controlled by a steep RanGTP gradient maintained across the nuclear envelope. Import receptors load cargoes in the absence of Ran in the cytoplasm, and RanGTP causes dissociation of the importin-cargo complex in the nucleus. Exportins form stable complexes with their cargoes in the presence of RanGTP, and cargo release is accomplished when the Ran-bound GTP molecule is hydrolyzed in the cytoplasm facilitated by concerted action of RanGAP and RanBP1 (RanBP2). Translocation of importins and exportins through the NPC occurs via binding to the FG-rich repeats of nucleoporins using their outer surface. RanGDP is transported from the cytoplasm back to the nucleus by nuclear transport factor 2 (NTF2), and reactivated by RCC1 (guanine exchange factor RanGEF) catalyzed nucleotide exchange [this figure was partially adapted from a figure created by Gorlich and Kutay (Gorlich and Kutay, 1999)]. 


\subsubsection{The admission ticket for the nucleus: NLSs}

Recognition of the import signals does not occur by a single uniform mechanism, and therefore no consensus import signal can be defined as proposed in the early days (Dingwall and Laskey, 1991). However, nuclear localization signals (NLSs) can be principally divided into two groups, the classical NLSs (cNLSs) and the nonclassical NLSs (ncNLSs). The large number of nuclear proteins bearing a cNLS are imported into the nucleus by the importin $\alpha / \beta$ heterodimer (reviewed in Gorlich and Mattaj, 1996; Melchior and Gerace, 1995; Nigg, 1997). In these importin-cargo complexes importin $\alpha$ represents the adaptor molecule that links the cargo with the actual import mediator importin $\beta$. Thus, importin $\alpha$ contains a cargo (cNLS) binding site (ARM repeats; see also chapter 1.4) and an importin $\beta$ binding (IBB) domain which consists of approximately $40 \%$ basic residues (Gorlich et al., 1996a; Weis et al., 1996b). cNLSs consist of short stretches of positively charged amino acids. They can be monopartite, as in the simian virus 40 (SV40) large-T antigen that consists of a heptapeptide containing five basic amino acids (Kalderon et al., 1984; Lanford and Butel, 1984) or bipartite, as in nucleoplasmin. The NLS in nucleoplasmin consists of two short basic clusters separated by a spacer of 10-amino acids (Dingwall and Laskey, 1991; Robbins et al., 1991). In addition to the cNLS-dependent pathway, importins can also function in the absence of adapter molecules like importin $\alpha$. In this alternative pathway the cargoes contain a ncNLS which is in general longer than the cNLS (for review, see Christophe et al., 2000). Proteins bearing ncNLSs directly bind to one of the 20 members of the importin $\beta$ family present in higher eukaryotes (Strom and Weis, 2001). The list of adapter independent cargoes is constantly growing and examples include the transcription factors CREB, Jun and Fos (Forwood et al., 2001), Smad-3 (Xiao et al., 2000), the retroviral proteins Rev (Henderson and Percipalle, 1997; Truant and Cullen, 1999) and Tat in HIV-1 (Truant and Cullen, 1999), the human T-cell leukemia virus Rex protein (Palmeri and Malim, 1999), the ribosomal proteins S7, L5, and L23a (Jakel and Gorlich, 1998), the core histones (Baake et al., 2001a; Mosammaparast et al., 2002b; Mosammaparast et al., 2001; Muhlhausser et al., 2001), and parathyroid hormonerelated protein (PTHrP) (Lam et al., 1999) which all directly bind to importin $\beta$. The paired-type homeodomain transcription factors Pax6, Pax3 and Crx (Ploski et al., 2004), the SUMO-1/ sentrin-conjugating enzyme hUBC9 and MGN binding protein RBM8 (Y14) (Mingot et al., 2001) bind directly to importin 13. Transportin mediates the nuclear import of M9-containing proteins such as hnRNP A1 (Pollard et al., 1996). The M9-domain, consisting of $\sim 38$ amino acid residues, is quite exceptional because it contains only a single essential basic residue, but is rich in glycine and comprises many aromatic residues (Bogerd et al., 1999). Besides recognizing M9domain containing proteins, transportin also mediates nuclear import of the ribosomal protein 
L23a (Jakel and Gorlich, 1998). The import signal in rpL23a, the so-called BIB domain, contains 43 amino acids (human) and is extremely basic having an isoelectric point of $\sim 12$. How does transportin bind to these very different import signals? The answer is rather simple; transportin has distinct, non-overlapping binding sites for the M9 and BIB domain. In addition to transportin, also importin $\beta$, importin 5, and importin 7 confer import of rpL23a (Jakel and Gorlich, 1998). The use of parallel import pathways applies also for its yeast homologue rpL25 which can choose between at least two transport factors, Yrb4p and Pse1p (Rout et al., 1997; Schlenstedt et al., 1997). This redundancy in nuclear transport pathways of essential proteins (cargoes) explains for instance why the majority of nuclear transport receptors are not essential in yeast ( $S$. cerevisiae). Observing redundant transport pathways often leaves the question open whether the same region/NLS is recognized by the individual import receptors or not. However, in the case of rpL23a it is known that importin $\beta$, transportin, importin 5 and importin 7 bind to the same region. Thus, this region is referred to as beta-like import receptor binding (BIB) domain (Jakel and Gorlich, 1998). The four import receptors share less than $15 \%$ sequence identity which makes this functional conservation quite remarkable. Finally, the binding sites in importin $\beta$ for cargoes correspond in general to the IBB domain binding site (HEAT 7-19, cargo binding site I) (Cingolani et al., 1999). Recently, the crystal structure of a fragment of importin $\beta$ bound to the ncNLS of the parathyroid hormone-related protein (PTHrP) revealed a second extended cargo binding site on importin $\beta$ (HEAT 1-11, cargo binding site II) (Cingolani et al., 2002). Since this second cargo binding site overlaps entirely with the RanGTP binding domain cargoes binding to this site must be released by RanGTP via a direct competition for the same binding site. In comparison, binding of RanGTP to importin $\beta$ induces a conformational change that triggers the release of cargoes bound to the IBB domain binding site.

However, with the exception of a few cases (e.g. IBB, BIB, and M9) the cargo recognition sites (minimal NLSs) have not been mapped in detail. Thus, despite the growing list of identified cargoes for the individual import receptors knowledge about the nature of NLSs remains rather limited.

\subsubsection{The easy way out: NESs}

As mentioned above, nuclear export is as important as nuclear import since many highly abundant substrates such as mRNAs (Izaurralde, 2002), tRNAs (Calado et al., 2002), translation factors (Bohnsack et al., 2002; Calado et al., 2002; Mingot et al., 2001), ribosomes (Johnson et al., 2002), and import adaptors (Kutay et al., 1997a) have to be exported from the nuclei (reviewed in Lei and Silver, 2002). So far, nuclear export receptors comprise the mRNA export 
factors (reviewed in Izaurralde, 2002) and seven importin $\beta$ related transport receptors. In contrast to importins, most exportins seem to be specialized on a narrow group of cargoes or even a single export substrate. For instance, CAS/exportin 2 recycles importin $\alpha$ adaptors to the cytoplasm (Kutay et al., 1997a), exportin-t mediates export of tRNA (Arts et al., 1998; Kutay et al., 1998), exportin 5 also exports tRNA and additionally the translation elongation factor 1A (eEF1A) (Bohnsack et al., 2002; Calado et al., 2002) and short microRNA precursors (premiRNAs) (Lund et al., 2004), exportin 4 exports eIF5A (Lipowsky et al., 2000), and exportin 6 keeps actin cytoplasmic (Stuven et al., 2003). Crm1/exportin 1 (Fornerod et al., 1997a; Stade et al., 1997) has so far been the only exception, however, the recently identified exportin 7 (Mingot et al., 2004) also appears to have a broad cargo specificity. Among the substrates recognized by Crm1 are several RNPs (ribosomes, SRP, U snRNAs) and a large number of proteins with leucine-rich nuclear export signals (NESs). CRM1 mediated nuclear export of ribosomal subunits such as the large (60S) subunit however requires sometimes shuttling adaptor proteins (such as NMD3 for the 60S subunit). Leucine-rich nuclear export sequences that mediate rapid export from the nucleus were originally identified in the viral protein HIV-1 Rev (Fischer et al., 1995 ) and the cAMP-dependent protein kinase inhibitor PKI (Wen et al., 1995). Leptomycin B (LMB), an antifungal cytotoxin isolated from a Streptomyces strain (Hamamoto et al., 1983), binds covalently to $\mathrm{Crm} 1$ and interferes with the formation of the heterotrimeric export complex (Fornerod et al., 1997a). Thus, it specifically inhibits the Crm1 export pathway. The use of LMB has subsequently led to the identification of many Crm1 export substrates that carry leucine-rich nuclear export signals. By comparing several functional NESs and random mutagenesis studies, a consensus motif for leucine-rich NESs was defined (Bogerd et al., 1996). However, this consensus motif does not allow a safe prediction of Crm1 cargoes based on their amino acid sequence alone. Examples show that even when a leucine-rich peptide sequence can direct export of a reporter protein and binds to Crm1 in vitro (Ossareh-Nazari et al., 1997), no safe conclusion can be drawn regarding its export function in the wild-type protein. In fact, the leucine-rich NES needs an appropriate protein context to fulfill its function, respectively to adopt a conformation that can be recognized by Crm1 (Paraskeva et al., 1999). To complicate matters even further, it recently was shown that nuclear export of 14-3-3 $\sigma$ (member of a larger protein family that regulates diverse cellular processes) is mediated by exportin 7 and not by Crm1 as previously suggested (Mingot et al., 2004). The authors further assume that numerous other export events which are currently credited to $\mathrm{Crm} 1$, might in fact be mediated by export receptors such as exportin 7 . The reason for their assumption is based, at least in part, on the use of LMB as sole assessment for defining nuclear export pathways. Crm1 is probably responsible 
for the cytoplasmic localization of RanGAP and RanBP1 (Richards et al., 1996). Thus, a block of the Crm1 export pathway by LMB treatment impairs or at least disturbs the RanGTP gradient and therefore ultimately inhibits all nuclear transport pathways parallel to the Crm1 export pathway. The nuclear export signals recognized by exportin 7 can also not be compared with the leucine-rich NESs and exportin 7 binds to their cargoes probably through multiple low affinity interactions (Mingot et al., 2004).

\subsection{Regulated nuclear transport: rule or exception?}

Proteins often contain both types of signals, NLS and NES, which allows them to shuttle between the nucleus and the cytoplasm. Changes in the import or export kinetics will then eventually determine the cargoes net transport. Hence, regulatory mechanisms must exist to alter kinetics. It turned out that the posttranslational modification of the cargo (primarily phosphorylation) is the key mechanism (reviewed in Kaffman and O'Shea, 1999; Mattaj and Englmeier, 1998; Schuller and Ruis, 2002).

\subsubsection{Specific transcription factors}

One of the best characterized examples of regulating the cargo-receptor interaction by cargo phoshorylation concerns the yeast transcription factor Pho4 (Kaffman et al., 1998a; Kaffman et al., 1998b; Komeili and O'Shea, 1999). Pho4 is the key regulator of the response to limiting amounts of inorganic phosphate. It could be shown that phosphorylation of Pho4 stimulates nuclear export which is mediated by Msn5 (yeast homologue to exportin 5) (Kaffman et al., 1998a) whereas only the unphosphorylated form of Pho4 is imported into the nucleus facilitated by Pse1 (Kap121, yeast homologue to importin 5) (Kaffman et al., 1998b). However, in the nucleus Pho4 activity is further regulated by Pho2. Only the Pho4/Pho2 heterodimer is a highly active transcription factor (Komeili and O'Shea, 1999).

Other ways to control interactions between cargoes and transport receptors include masking of NLSs and/or NESs. For instance, nuclear transport studies on the transcription factors p53 and NF-AT established NES masking as major regulatory mechanism which might be also used by other shuttling proteins. In detail, in response to stress p53 moves into the nucleus, gets stabilized and induces cell cycle arrest. Thereby, p53 uses tetramerization, promoted by phosphorylation, to mask its NES (Stommel et al., 1999). In contrast to this homooligomerization, heterodimer formation with Mdm2 enhances the relatively weak NESs found in both proteins resulting in nuclear export of the heterodimer (Henderson and Eleftheriou, 2000). In the case of NF-AT, a transcriptional activator whose localization is regulated in response to 
the intracellular calcium levels, nuclear export is controlled by binding of calcineurin to the NESs (Zhu and McKeon, 1999). In fact, when intracellular calcium levels increase, the phosphatase calcineurin is activated and binds to NF-AT which as a consequence will be dephosphorylated. This unmasks the NLS of NF-AT and the protein is translocated into the nucleus. However, when NF-AT enters the nucleus, Crm1-dependent export is inhibited because its NES is masked by the activated, bound calcineurin. Taken together, binding of calcineurin simultaneously triggers unmasking of the NLS (by dephosphorylation) and masking of the NES (through inhibitory binding). Further, this example shows that phosphorylation may also regulate the masking of NLSs.

\subsubsection{General transcription factors}

In contrast to the nuclear transport of many specific transcription factors, the nuclear transport and therefore the activity of general transcription factors appears not to be regulated (additionally controlled by their subcellular localization). For the transcription of genes by RNA polymerase II a so called pre-initiation complex (PIC) has to be formed at the promoter (for review, see Burley and Roeder, 1996). This complex consists of many general transcription factors such as TFIIA, TFIIB, TFIID, TFIIE, and TFIIF. TFIID is comprised of the TATA-binding protein (TBP) and TBP-associated factors (TAFs) (Orphanides et al., 1996). Besides RNA polymerase II transcription, TBP is also essential for RNA polymerase I and III transcription. (Hernandez, 1993) and represents the only sequence specific DNA binding factor of the general transcription machinery (Burley and Roeder, 1996).

The transport mechanisms used to import TFIIA and TBP have been revealed in yeast indicating that Kap114p is the transport receptor of TBP (Morehouse et al., 1999; Pemberton et al., 1999), while the nuclear import of TFIIA is mediated by Kap122p (Pdr6) (Titov and Blobel, 1999). Surprisingly, TBP and TFIIA, being essential proteins in yeast (deletion of TBP/TFIIA is lethal), are transported into the nucleus by non-essential import receptors (deletion of Kap114p/Kap122p is not lethal). This of course implies that alternative pathways must exist to import these two general transcription factors. The interaction of TBP with three other import receptors (Pemberton et al., 1999) indeed suggests that multiple pathways for the nuclear import of TBP and TFIIA exist. It should be mentioned that only four members of the importin $\beta$ family present in yeast are encoded by essential genes (Kap95p, importin $\beta$; Kap121p, importin 5; Crm1p, Crm1/exportin1; Cse1p, CAS) (Pemberton et al., 1999 and references therein). 


\subsection{Exceptions to the importin $\beta$ family pathways}

Although almost all known nuclear transport pathways are mediated by importin $\beta$ family members, there are exceptions. One example has already been mentioned, nuclear import of RanGDP is facilitated by NTF2 (Ribbeck et al., 1998). Another example is the nuclear export of mRNAs which are apparently not exported as naked RNAs but as RNP complexes. Formation of heterodimers consisting of TAP/Mex67(yeast homologue) and p15(human)/Mtr2(functional analogue in yeast) allows export of mRNA from the nucleus to the cytoplasm (Braun et al., 2001; Braun et al., 2002; Strasser et al., 2000) (for review, see Conti and Izaurralde, 2001; Lei and Silver, 2002; Weis, 2002). All of these nuclear transport factors can cross the nuclear envelope bi-directionally, most likely through specific interactions with FG-rich repeats of the NPC.

However, it was also shown that individual proteins do not follow the general rules and are able to passage through the NPC by interacting directly with the nucleoporins. Examples for this exceptional behavior are $\beta$ catenin, HIV Vpr, and hnRNP K. The latter two proteins contain cNLSs which enable them to interact with importin $\alpha$, however, they also bind directly to nucleoporins and translocate through the NPC independently of soluble transport receptors (for review, see Gorlich and Kutay, 1999).

Besides their described roles in nuclear transport importin $\alpha$, importin $\beta$ and in particular Ran fulfill multiple cellular roles during mitosis. For instance, Ran appears to be the key regulator for the mitotic microtubule network. By counteracting importin $\alpha$ and $\beta$, RanGTP permits mictrotubule nucleation and stabilization around chromosomes. In fact, spindle assembly factors such as TPX2, NuMA, and XCTK2 are continuously bound by importin $\alpha / \beta$ dimers which inhibits the function of these factors. In the vicinity of chromosomes there is a high RanGTP concentration that relieves inhibition and allows localized spindle assembly (Weis, 2003). Besides being a positional marker for the eukaryotic genome, Ran is further required for nuclear envelope formation around chromatin (reviewed in Harel and Forbes, 2004; Weis, 2003).

To conclude, Ran has a key role in regulating cellular processes throughout the cell cycle, and transport of macromolecules between the cytoplasm and the nucleus is mediated by at least three different classes of soluble transport receptors. Beyond question, most of the work is hereby done by members of the importin $\beta$ family, while the families of TAP/Mex67 and NTF2-like factors (such as p15) comprise groups of carriers that are distinct from the importin $\beta$ family. 


\subsection{Histones and their nuclear transport}

\subsubsection{From structure to function}

A hallmark of all eukaryotes is the organization of DNA in the form of a nucleoprotein complex known as chromatin. Histones are the major structural proteins of chromatin. They allow the compaction of DNA, and neutralize the electrostatic character of the DNA. This group of basic proteins comprises the four core histones $\mathrm{H} 2 \mathrm{~A}, \mathrm{H} 2 \mathrm{~B}, \mathrm{H} 3, \mathrm{H} 4$ and the $\mathrm{H} 1$ linker histones (Allan et al., 1986; Pruss et al., 1996; Wolffe, 1998). Two copies of each core histone protein H2A, H2B, H3, and H4 are assembled into a histone octamer. About 146 base pairs (bp) of DNA wrap (lefthanded) around the surface of the histone octamer and form the nucleosomal core (Luger et al., 1997). The repeating nucleosome cores are connected (linked) by approximately 50 bp linker DNA. H1 linker histones interact with the linker DNA and stabilize a higher order chromatin structure. In conclusion, the nucleosome consisting of nucleosome core, linker DNA, and H1 represents the fundamental repeating unit of eukaryotic chromatin and determines the DNA accessibility (for review, see Kornberg and Lorch, 1999).

Histones are rather small, very basic proteins. The linker histone is highly enriched in lysine, $\mathrm{H} 2 \mathrm{~A}$ and $\mathrm{H} 2 \mathrm{~B}$ are moderately lysine rich, and $\mathrm{H} 3$ and $\mathrm{H} 4$ are rich in arginine. The size of core histones varies between $11 \mathrm{kDa}$ and $15 \mathrm{kDa}$, whereas the $\mathrm{H} 1$ histones have a size of about $22 \mathrm{kDa}$. With regard to their structure the four core histones show an overall similarity, consisting of unstructured, basic amino- and carboxy terminal domains and a hydrophobic, central globular domain that forms the histone fold motif (HFM) (Arents and Moudrianakis, 1995; Baxevanis et al., 1995; Luger et al., 1997). The HFM is composed of three/four $\alpha$-helices separated by short loop regions and is involved in histone-histone and histone-DNA interactions. In the latter case, the HFM accounts directly for the organization of $121 \mathrm{bp}$ of DNA around the histone octamer (Luger et al., 1997). Presumably, because of its unique DNA-binding properties the HFM is highly conserved across different species and considered to be a ubiquitous architectural motif for DNA compaction and protein dimerization (Arents and Moudrianakis, 1995). In contrast to the central globular domains of the core histones, which are responsible for histone-histone interactions within the core, the amino-terminal tails of the core histones spread out from the nucleosome core. Posttranslational modifications of the positively charged aminoterminal tails modulate the chromatin structure and therefore play an important role in all cellular processes that require access to the DNA (Strahl and Allis, 2000). Thus, transcription, replication, and recombination are associated with acetylation, phosphorylation, and methylation of the amino-terminal histone tails. 
The process of chromatin/nucleosome assembly is facilitated by a number of different histone chaperones and chromatin assembly factors; however, the exact mechanisms how many of these factors function are not fully understood (Adams and Kamakaka, 1999; Verreault, 2000). Anyway, the assembling of a histone octamer involves the deposition of a $(\mathrm{H} 3-\mathrm{H} 4)_{2}$ tetramer (can be described as dimer of a dimer) onto DNA, which constitutes the first step of nucleosome assembly. The subsequent addition of a pair of $\mathrm{H} 2 \mathrm{~A}-\mathrm{H} 2 \mathrm{~B}$ heterodimers leads then to the formation of the histone octamer (Adams and Kamakaka, 1999; Ito et al., 1997; Verreault, 2000). Although the question of whether the formation of these histone heterodimers (oligomers) takes place in the cytoplasm or in the nucleus has not been completely solved yet, nuclear import of histones is clearly a prerequisite for their deposition onto DNA and the assembly of chromatin.

\subsubsection{Nuclear import of histones}

Histones, as all nuclear proteins encoded in nuclear DNA, are synthesized in the cytoplasm and need to cross the nuclear envelope to fulfill their nuclear function. Thus, histones are among the most abundant substrates for nuclear transport during the $\mathrm{S}$ phase of the cell cycle because a vast amount of histones is needed for the formation of new nucleosomes on replicated DNA. Since histones are smaller than the exclusion size of the NPC it could not be completely excluded that they enter the nucleus by diffusion. However, it was shown that histones are transported into the nucleus in a signal dependent, energy dependent, receptor-mediated process through the NPC (Breeuwer and Goldfarb, 1990; Kurz et al., 1997; Schwamborn et al., 1998).

Further, it was revealed that a number of different transport receptors are involved in the nuclear import of histones (Baake et al., 2001a; Bauerle et al., 2002; Jakel et al., 1999; Mosammaparast et al., 2002b; Mosammaparast et al., 2001; Muhlhausser et al., 2001). In case of the core histones redundant transport pathways exist, whereas specific importins even serve as primary or predominant receptors, at least in yeast (Mosammaparast et al., 2002b; Mosammaparast et al., 2001). The biochemical mechanism via which the specific importins become preferred transport receptors remains still unclear. Despite their relation, the import pathways of the linker histones and the core histones are rather different. H1 linker histones are imported into the nucleus in an energy-dependent process that is facilitated by an importin $\beta$-importin 7 heterodimer (Jakel et al., 1999). Similar to the importin $\alpha / \beta$ heterodimer for proteins containing a cNLS, heterodimerization of importin $\beta$ with importin 7 is essential and sufficient for their function as an import receptor for H1 linker histones (Bauerle et al., 2002; Jakel et al., 2002). However, in this particular case the heterodimeric importin complex does not only function as an import mediator but has an additional chaperone-like function in covering extended basic domains. 
The nuclear import of core histones differs from that of the linker histones and other nuclear proteins containing cNLSs. In vitro binding and reconstitution experiments with recombinant transport factors as well as comprehensive in vivo analysis in yeast demonstrated that multiple pathways contribute to the nuclear import of the four core histones. In mammalian cells, at least five members of the importin $\beta$ superfamily (namely importin $\beta$, importin 5 , importin 7 , importin 9 and transportin) mediate the nuclear uptake of the core histones (Baake et al., 2001a; Muhlhausser et al., 2001). In yeast, histones H2A and H2B utilize Kap114p (importin 9 homologue) as their primary transport receptor (Mosammaparast et al., 2001), while Kap123p (importin 4 homologue) fulfills this function for H3 and H4 (Mosammaparast et al., 2002b). Additionally, Kap121p (importin 5 homologue) was found to serve as secondary receptor for all of the core histones.

The role of the histone chaperone NAP1 (nucleosome assembly factor 1) in the nuclear transport of the core histones $\mathrm{H} 2 \mathrm{~A}$ and $\mathrm{H} 2 \mathrm{~B}$, however, still remains unclear. On one hand, it was reported that NAP1 bridges the interaction between the amino-terminal tails of $\mathrm{H} 2 \mathrm{~A} / \mathrm{H} 2 \mathrm{~B}$ and the import receptor Kap114p (Mosammaparast et al., 2002a). On the other hand, it was demonstrated that NAP1 has a preference for the $(\mathrm{H} 3-\mathrm{H} 4)_{2}$ tetramer and does not bind to the amino-terminal tails of H2A/H2B (McBryant et al., 2003). Furthermore, it has been clearly shown that import receptors directly interact with the core histones and that this direct interaction is sufficient to confer nuclear import in vitro and in vivo (Baake et al., 2001a; Mosammaparast et al., 2002b; Mosammaparast et al., 2001; Muhlhausser et al., 2001).

\subsection{The cargo of interest: NF-Y}

One of the most ubiquitous promoter elements in higher eukaryotes is the so-called CCAAT box which can be found in about $30 \%$ of the promoters (Bucher, 1990). CCAAT boxes are localized in close vicinity of other promoter elements (such as the TATA box) and are extremely conserved within promoters of the homologous genes across species; in terms of position, orientation and the nucleotides flanking the central CCAAT-pentanucleotide. The only exception to this broad distribution is the Drosophila melanogaster genome in which promoters are devoid of this element.

The CCAAT binding factor (CBF), also named NF-Y because it has been originally identified as a protein that binds to the major histo-compatibility complex (MHC) class II Ea promoter Y box, can be considered as the major CCAAT-binding activator. The key role of NF-Y is to act synergistically with other transcription factors for activation (reviewed in Maity and de Crombrugghe, 1998; Mantovani, 1999). Genes controlled by NF-Y include developmentally 
controlled and tissue specific genes (Berry et al., 1992; Ronchi et al., 1996), housekeeping and inducible genes (Marziali et al., 1997; Roy and Lee, 1995), cell cycle regulated genes, such as $C D C 2, C D C 25 A / B / C$, cyclin A2, cyclin B1/B2, E2F1 (Caretti et al., 2003), and replicationdependent histone H3 (Koessler et al., 2004). Furthermore, NF-Y mediated transcription is required for cell proliferation and viability (Bhattacharya et al., 2003).

NF-Y is a heterotrimeric complex composed of three subunits, NF-YA, NF-YB, and NF-YC which are all essential for CCAAT binding (McNabb et al., 1995; Sinha et al., 1995). Each subunit contains a core region that has been highly conserved throughout evolution. The conserved segments of the two subunits NF-YB and NF-YC contain a histone fold motif (HFM) as main structural feature (Arents and Moudrianakis, 1995; Baxevanis et al., 1995). The HFM containing subunits NF-YB and NF-YC belong to the H2A-H2B subfamily sharing low amino acid sequence identity but high structural resemblance regarding the HFM.
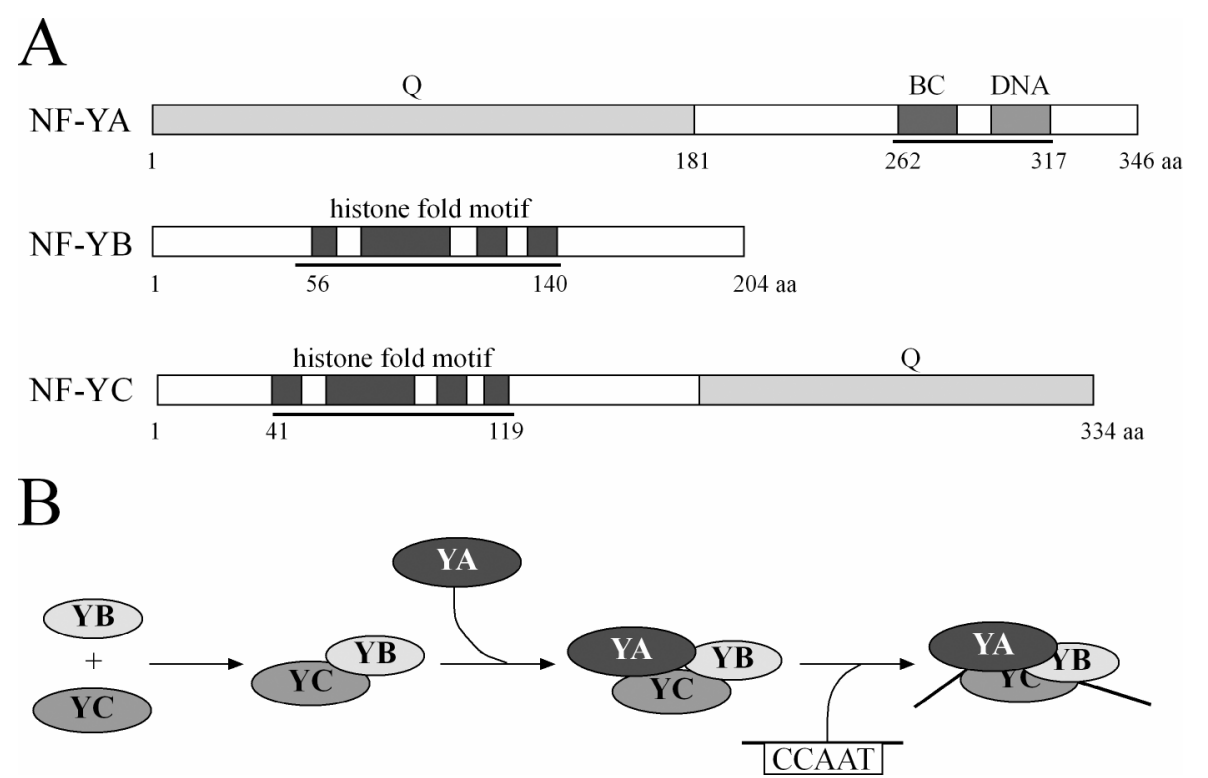

FIG. 3. NF-Y subunits and their association. (A) Schematic representation of the NF-Y proteins. All three subunits contain evolutionarily conserved core regions (represented by the bar below the schemes). In case of NF-YB and NF-YC the conserved regions comprise a histone fold motif (HFM) (black boxes indicate the positions of the four $\alpha$-helices of the HFM). The NF-YA core region is 56 amino acids long, and can be divided into N-terminal subunit interaction domain (BC) and C-terminal DNA binding domain (DNA). In addition, NF-YA and NF-YC contain large glutamine-rich sequences $(\mathrm{Q})$ that function as secondary activation domains. (B) Association of the NF-Y subunits and specific binding to DNA. NF-YB and NF-YC dimerize head to tail via their HFMs, a prerequisite for association of NF-YA. Once the heterotrimeric complex is formed, it binds DNA (CCAAT boxes) with very high specificity and affinity. The mode of protein interactions between the three subunits is similar in higher and lower eukaryotes [this figure was partially adapted from a figure created by Mantovani (Mantovani, 1999)]. 
NF-YB and NF-YC dimerize tightly head to tail via their HFMs, which offers a complex surface necessary for association of NF-YA. NF-YA is not able to interact with either NF-YB or NF-YC alone (Kim et al., 1996b; Sinha et al., 1996; Sinha et al., 1995), and the mode of protein interactions between the subunits appears to be similar in higher and lower eukaryotes. The resulting heterotrimeric complex has a high affinity and sequence specificity for the CCAAT boxes (Bi et al., 1997; Dorn et al., 1987; Kim and Sheffery, 1990).

Most of the sequence-specific interactions of the NF-Y trimer are made by NF-YA (for review, see Mantovani, 1999) which indirectly could be confirmed by the structural analysis of the NF-YB/NF-YC dimer (Romier et al., 2003). In more detail, the NF-YB/NF-YC structure was superimposed onto the $\mathrm{H} 2 \mathrm{~A} / \mathrm{H} 2 \mathrm{~B}$ dimer (nucleosome core) and no residue from the HFMs of NF-YB and NF-YC could be identified that would be able to make specific contacts with the CCAAT box.

In contrast to NF-YB and NF-YC the evolutionarily conserved domain of NF-YA does not contain a structural feature such as the HFM. Instead, the conserved region is 56 amino acids long, localized at the C-terminus of the protein and can be divided into two distinct parts separated by a short spacer. The N-terminal part is responsible for contacting the NF-YB/NF-YC histone fold pair (subunit interaction domain) and the C-terminal part has been shown to be essential for contacting DNA (DNA interaction domain). In addition, NF-YA and NF-YC contain large glutamine-rich sequences that function as secondary activation domains.

Another feature of the HFM containing subunits is that they can obviously act as dimer (independent of NF-YA) and fulfill functions in the absence of the regulatory subunit NF-YA. For example, NF-YB/NF-YC heterodimers can efficiently associate with DNA during nucleosome formation and thereby have an intrinsic affinity for H3-H4 (Caretti et al., 1999). Further, activation assays with HFM containing subunits fused to GAL4 also indicated that they are sufficient to activate transcription robustly, two- to fourfold better than the NF-Y trimer (de Silvio et al., 1999), and biochemical evidence suggests that NF-YB and NF-YC are associated with different complexes of high molecular weight in the absence of NF-YA (Bellorini et al., 1997).

\subsection{The goals of this study: What was known and what did we want to find out?}

With regard to the nuclear transport of the core histones, it had been shown that multiple pathways can contribute to the nuclear import of the four different core histones. Thus, the nuclear import receptors of the cores histones had been identified in yeast (Mosammaparast et al., 2002b; Mosammaparast et al., 2001) and mammals (Baake et al., 2001a; Muhlhausser et al., 
2001). Further, previous studies in our group had revealed that both the basic amino-terminal tails and the central globular domains of the four core histones can direct nuclear import of a reporter protein ( $\beta$-galactosidase fusion proteins) (Baake et al., 2001b). While the aminoterminal tails of the core histones $\mathrm{H} 2 \mathrm{~A}$ and $\mathrm{H} 2 \mathrm{~B}$ had been characterized as nuclear localization signals in yeast (Mosammaparast et al., 2001) one aim of this study was to determine the role of the main structural element of the central globular domains, the histone fold motif, in the nuclear import of the different core histones.

The structural feature of a histone fold motif, however, can not only be found in histones but is shared by a number of different proteins. NF-YB and NF-YC, two subunits of the heterotrimeric CCAAT-specific transcription factor NF-Y, belong to the $\mathrm{H} 2 \mathrm{~A} / \mathrm{H} 2 \mathrm{~B}$ subfamily and also contain this structural motif. Using the histone fold motif as possible bridge, the main aim of this project was to characterize the nuclear transport pathway of the transcriptional activator NF-Y. In detail, the nuclear transport mechanisms of the three different NF-Y subunits NF-YA, NF-YB, and NF-YC had to be analyzed. Methods that should be applied to investigate the transport mechanisms included cell culture experiments, in vitro binding studies and nuclear import assays as well as microinjection experiments into Xenopus oocytes. 


\section{Materials and methods}

\subsection{Materials}

\subsubsection{Organisms}

\subsubsection{Bacterial strains}

The following Escherichia coli (E. coli) strains have been used:

\section{Cloning strains:}

$\underline{\text { Top10 }}$ (Invitrogen, Karlsruhe)

Top10 E. coli are ideal for high-efficiency cloning and plasmid propagation. They allow stable replication of high-copy number plasmids. The genotype of Top10 cells is similar to the DH10B ${ }^{\mathrm{TM}}$ strain, and offers $h s d \mathrm{R}$ for efficient transformation of non-methylated DNA.

Genotype: $\quad \mathrm{F}^{-}$mcrA $\Delta(m r r-h s d \mathrm{RMS}-m c r \mathrm{BC})$ Ф80lacZ $\Delta \mathrm{M} 15 \Delta$ lacX74 recA1 ara $\Delta 139$

$$
\Delta(\text { ara-leu }) 7697 \text { galU galK rpsL }\left(\mathrm{Str}^{\mathrm{R}}\right) \text { end } \mathrm{A} 1 \text { nup } \mathrm{G}
$$

Protein expression strains:

$\underline{\text { BL21 (DE3) }}$ (Stratagene, Heidelberg)

The DE3 cells carry a chromosomal copy of the T7 RNA polymerase gene under control of the lacUV5 promoter. Thus, this strain has been used for high-level expression of genes cloned into expression vectors that contain a bacteriophage $\mathrm{T} 7$ promoter (such as $\mathrm{pET}$ vectors, for which this strain has been used in particular).

Genotype: $\quad \mathrm{F}^{-}$ompT $h s d S_{B}\left(r_{B}^{-} m_{B}^{-}\right) \operatorname{gal} d c m(\mathrm{DE} 3)$

\section{BL21 (DE3) pLysS (Novagen - Merck Biosciences, Bad Soden)}

Same as above but has an additional vector transformed that produces a small amount of T7 lysozyme that is used to suppress basal expression of T7 RNA polymerase prior to induction and thus reduces 'leaky' expression.

Genotype: $\quad \mathrm{F}^{-}$ompT $h s d S_{B}\left(r_{B}^{-} m_{B}^{-}\right)$gal dcm (DE3) pLysS $\left(\mathrm{Cm}^{\mathrm{R}}\right)$

$\underline{\text { XL1-blue }}$ (Stratagene, Heidelberg)

Genotype: $\quad \operatorname{Tn} 10\left[\mathrm{~F}^{\prime}\right.$ proAB lacl $\left.{ }^{q} \mathrm{Z} \Delta \mathrm{M} 15\right]$ recA1 endA1 gyrA96 thi-1 hsdR17( $\left.\mathrm{r}_{\mathrm{K}}{ }^{-} \mathrm{m}_{\mathrm{K}}{ }^{+}\right)$sup $\mathrm{E} 44$ relA1 lac 
$\underline{\text { JM109 }}$ (Stratagene, Heidelberg)

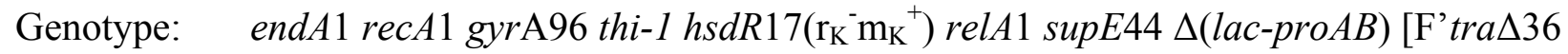
proAB $\left.\operatorname{lacl}^{q} \mathrm{Z} \Delta \mathrm{M} 15\right]$

XL1 Blue and JM109 are E. coli strains harboring the lacI ${ }^{\mathrm{q}}$ mutation and therefore can be used as expression hosts for expressing (nontoxic) proteins. lacI $^{\mathrm{q}}$ is a mutation of lacI that produces very high levels of the lac repressor to efficiently block transcription. In theory, however, expression is regulated less tightly than in strains harboring the pREP4 plasmid. Thus, expression of proteins which are toxic to the cell may result in poor culture growth due to 'leaky' expression before induction with IPTG.

M15[pREP4] (Qiagen, Hilden)

Genotype: $\quad \mathrm{Nal}^{S} \mathrm{Str}^{S} \mathrm{Rif}^{S} \mathrm{Lac}^{-} \mathrm{Ara}^{-} \mathrm{Gal}^{-} \mathrm{Mtl}^{-} \mathrm{F}^{-} \mathrm{RecA}^{+} \mathrm{Uvr}^{+} \mathrm{Lon}^{+}$

In the M15[pREP4] trans system, the M15 host strain contains the low-copy plasmid pREP4 which confers kanamycin resistance and constitutively expresses the lac repressor protein encoded by the lacI gene (Farabaugh, 1978). Only the presence of high levels of the lac repressor protein can efficiently regulate and repress the extremely high transcription rate initiated at the T5 promoter.

\subsubsection{Animals}

Xenopus laevis ('the African clawed frog') belongs to the family of Pipidae, subfamily Xenopinae. The frogs were ordered from the company Nasco (Fort Atkinson, Wisconsin, USA), and were treated according to the animal laws in the Department of Developmental Biochemistry (University of Göttingen).

\subsubsection{Cell lines}

HeLa cells were obtained from the German Collection of Microorganisms and Cell Cultures (Deutschen Sammlung von Mikroorganismen und Zellkulturen, Braunschweig) DSMZ No: ACC57. HeLa cells are human cervix carcinoma cells which grow epithelial-like in monolayers.

\subsubsection{Chemicals}

Acetic acid (Roth, Karlsruhe)

Acetone (Roth, Karlsruhe)

Acrylamide solution (30\% (w/v) acrylamide, $0.8 \%(\mathrm{w} / \mathrm{v})$ bisacrylamide) (Roth, Karlsruhe) Adenosine-5'-diphosphate (ADP) (Sigma, Deisenhofen) 
Adenosine-5' -triphosphate (ATP) (Sigma, Deisenhofen)

Agarose (Roth, Karlsruhe)

Ammonium acetate (Merck, Darmstadt)

Ammonium chloride (Merck, Darmstadt)

Ammonium peroxosulfate (APS) (Merck, Darmstadt)

Apyrase (Sigma, Deisenhofen)

Bacto-agar (AppliChem, Darmstadt)

Bacto-tryptone (AppliChem, Darmstadt)

Bacto-yeast extract (AppliChem, Darmstadt)

Boric acid (Roth, Karlsruhe)

Bovine serum albumin (BSA) (Sigma, Deisenhofen)

Bromophenol blue (Serva, Heidelberg)

$\mathrm{CaCl}_{2}$ (Merck, Darmstadt)

Collagenase (Sigma, Deisenhofen)

Coomassie Brilliant Blue G-250 (Merck, Darmstadt)

Coomassie Brilliant Blue R-250 (Merck, Darmstadt)

Creatine phosphokinase (Sigma, Deisenhofen)

Deoxyribonucleoside triphosphates (dNTPs) (Roche, Mannheim)

Diamino-2-phenylindol (DAPI) (Sigma, Deisenhofen)

Digitonin (Calbiochem, Bad Soden)

Dithiothreitol (DTT) (Biomol, Hamburg)

DMSO (Sigma, Deisenhofen)

EDTA (disodium ethylenediaminetetraacetate) (Serva, Heidelberg)

EGTA (ethylene-bis(oxyethylenenitrilo)tetraacetic acid) (Serva, Heidelberg)

Ethanol (Merck, Darmstadt)

Ethidium bromide (Sigma, Deisenhofen)

Fetal calf serum (FCS) (PAA, Cölbe)

Fluorescein isothiocyanate (FITC) (Sigma, Deisenhofen)

Fluorescein-5-maleimide (Pierce, Rockford, USA)

FLUOS (carboxy fluorescein $N$-hydroxysuccinimide ester) (Roche, Mannheim)

Glycerol (Serva, Heidelberg)

Glycine (Serva, Heidelberg)

Guanosine-5'-diphoshate (GDP) (Sigma, Deisenhofen)

Guanosine-5'-triphoshate (GTP) (Sigma, Deisenhofen) 
$\mathbf{H}_{2} \mathrm{O}$ (HPLC purity) (Merck, Darmstadt)

$\mathrm{H}_{2} \mathrm{SO}_{4}$ (Merck, Darmstadt)

$\mathrm{HCl}$ (Merck, Darmstadt)

HEPES ( $N$-2-hydroxyethylpiperazine- $N$ '-2-ethanesulfonic acid) (Serva, Heidelberg)

Imidazole (Roth, Karlsruhe)

IPTG (isopropylthio-ß-D-galactoside) (Peqlab, Erlangen)

Isopropanol (Merck, Darmstadt)

$\mathbf{K}_{2} \mathrm{HPO}_{4}$ (Merck, Darmstadt)

$\mathrm{KCl}$ (Merck, Darmstadt)

$\mathrm{KH}_{2} \mathrm{PO}_{4}$ (Merck, Darmstadt)

Lysozyme (Sigma, Deisenhofen)

Magnesium acetate (Serva, Heidelberg)

MEM (Modified Eagle's Medium) (Biochrom, Berlin)

Methanol (Serva, Heidelberg)

$\mathrm{MgCl}_{2}$ (Merck, Darmstadt)

$\mathrm{Na}_{2} \mathrm{HPO}_{4}$ (AppliChem, Darmstadt)

$\mathrm{NaH}_{2} \mathrm{PO}_{4}$ (Merck, Darmstadt)

$\mathrm{NaHCO}_{3}$ (Merck, Darmstadt)

$\mathrm{NaOH}$ (Merck, Darmstadt)

NP-40 (Nonidet P-40) (Merck, Darmstadt)

Paraformaldehyde (Serva, Heidelberg)

Phosphocreatine (Sigma, Deisenhofen)

Phosphoric acid (Merck, Darmstadt)

Potassium acetate (Serva, Heidelberg)

Reticulocyte lysate (Promega, Mannheim)

SDS (sodium dodecyl sulfate) (Serva, Heidelberg)

Sodium acetate (Merck, Darmstadt)

Sodium azide $\left(\mathrm{NaN}_{3}\right)$ (Roche, Mannheim)

TEMED (N,N,N',N'-tetramethylethylenediamine) (Serva, Heidelberg)

Trichloroacetic acid (TCA) (Merck, Darmstadt)

Tris [Tris(hydroxymethyl)aminomethane] (Roth, Karlsruhe)

TRITC (Tetramethylrhodamine isothiocyanate) (Sigma, Deisenhofen)

Triton X-100 (Merck, Darmstadt)

Tween-20 (Sigma, Deisenhofen) 
Vectashield (Mounting medium with DAPI) (Vector Laboratories, Burlingame, USA)

Wheat germ agglutinin (WGA) (Sigma, Deisenhofen)

$\beta$-mercaptoethanol (BME) (Serva, Heidelberg)

\subsubsection{Antibodies}

Primary antibodies:

Monoclonal mouse anti-NF-YA (BD Pharmingen)

Polyclonal rabbit anti-CBF-A (Santa Cruz)

Polyclonal rabbit anti-CBF-C (Santa Cruz)

Monoclonal mouse anti- $\beta$-galactosidase (Sigma)

Monoclonal mouse anti-HA (Santa Cruz)

Monoclonal mouse anti-Myc (Invitrogen)

Monoclonal mouse anti-GST (Santa Cruz)

Secondary antibodies:

Cy3-labeled goat anti-mouse (Dianova)

AlexaFluor488-labeled goat anti-mouse (Molecular Probes)

AlexaFluor555-labeled goat anti-mouse (Molecular Probes)

AlexaFluor488-labeled goat anti-rabbit (Molecular Probes)

AlexaFluor555-labeled goat anti-rabbit (Molecular Probes)

\subsubsection{Protease inhibitors}

Aprotinin (Roche, Mannheim)

Complete ${ }^{\circledR}$ (EDTA free) protease inhibitor cocktail (pills) (Roche, Mannheim)

Leupeptin (Roche, Mannheim)

Pepstatin (Roche, Mannheim)

Phenylmethylsulfonyl fluoride (PMSF) (Serva, Heidelberg)

\subsubsection{Enzymes}

Alkaline Phosphatase, calf intestinal (CIP) (1 U/ $\mu$ l) (Roche, Mannheim)

Lysozyme (Muramidase), stock solution $50 \mathrm{mg} / \mathrm{ml} \mathrm{H}_{2} \mathrm{O}$ (Sigma, Deisenhofen)

Restriction enzymes (New England Biolabs, Schwalbach; MBI Fermentas, St.Leon-Rot)

RNase A, stock solution $10 \mathrm{mg} / \mathrm{ml}$ (Sigma, Deisenhofen)

RNAsin (40 U/ $\mu 1)$ (Promega, Mannheim)

T4 DNA-Ligase (1 U/ $\mu$ l) (New England Biolab, Schwalbach) 
T4 DNA-polymerase (3 U/ $\mu$ l) (New England Biolabs, Schwalbach)

T4 polynucleotide kinase (10 U/ $\mu$ l) (New England Biolabs, Schwalbach)

Trypsin (Biochrom, Berlin)

\section{Thermophilic DNA polymerases}

The thermophilic DNA polymerases, like other DNA polymerases, catalyze template-directed synthesis of DNA from nucleotide triphosphates. A primer having a free 3' hydroxyl is required to initiate synthesis. In addition also magnesium ions are necessary. In general, they have maximal catalytic activity at $75^{\circ} \mathrm{C}$ to $80^{\circ} \mathrm{C}$.

RedTaq $^{\mathrm{TM}}$ (from Thermus aquaticus) DNA-Polymerase (1 U/ $\mu$ l) (Sigma, Deisenhofen) Vent $_{\mathrm{R}}{ }^{\circledR}$ (from Thermococcus litoralis) DNA-Polymerase (2 U/ $\mu$ ) (New England BioLabs, Schwalbach)

Note, the Taq polymerase lacks $3^{\prime} \rightarrow 5^{\prime}$ proofreading exonuclease activity and therefore has a higher error rate compared to the polymerases with exonuclease activity such as Vent ${ }_{R}{ }^{\circledR}$ polymerase. The total error rate of Taq polymerase has been reported between $1 \times 10^{-4}$ to $2 \times 10^{-5}$ errors per base pair (bp).

\subsubsection{Kits}

Big Dye ${ }^{\mathrm{TM}}$ Terminator Sequencing Ready Reaction (Applied Biosystems, Foster City, USA)

E.Z.N.A Cycle-Pure Kit (Peqlab, Erlangen)

E.Z.N.A. Plasmid-Miniprep Kit II (Peqlab, Erlangen)

QIAEX ${ }^{\circledR}$ II Agarose Gel Extraction Kit (Qiagen, Hilden)

Qiagen Plasmid-Maxi/Midi-Kit (Qiagen, Hilden)

$\mathrm{T}_{\mathrm{N}} \mathrm{T}$ coupled reticulocyte lysate system (Promega, Mannheim)

Wizard Plus SV Minipreps (Promega, Mannheim)

Effectene $^{\mathrm{TM}}$ Transfection Reagent (Qiagen, Hilden)

NucleoSpin ${ }^{\circledR}$ Plasmid (Macherey-Nagel, Düren)

\subsubsection{Gel filtration columns}

NAPTM_5 column (Amersham Biosciences, Freiburg)

Superdex ${ }^{\circledR} 200$ HiLoad 16/60 column (Amersham Biosciences, Freiburg)

Superdex ${ }^{\circledR} 75$ HiLoad 16/60 column (Amersham Biosciences, Freiburg) 
Vivaspin (Vivascience; Biofiltronics, Nörten-Hardenberg)

\subsubsection{Filters and membranes}

Folded filters (Ø12.5 cm) (Macherey-Nagel, Düren)

Filters 2668 (Schleicher \& Schüll, Dassel)

\subsubsection{Molecular weight standards}

\section{$\underline{\text { DNA }}$}

1 kb DNA ladder (Invitrogen, Karlsruhe)

$\lambda$-DNA size markers (digested HindIII or EcoRI-HindIII) (MBI Fermentas, St.Leon-Rot)

$\underline{\text { Protein }}$

Dual-Color Precision broad range marker (BioRad, München)

SDS-PAGE Molecular weight standard broad range marker (BioRad, München)

\subsubsection{Liquid media and solutions}

Most of the liquid media and the buffers used were prepared essentially as described in Sambrook et al., 1989. In general, deionized water was used to prepare solutions which were then sterilized by autoclaving for 20 minutes at $110-120^{\circ} \mathrm{C}$. In case of heat-sensitive substances the solutions were sterilized by filtration through a 0.2 micron filter (pore size $0.2 \mu \mathrm{m}$ ). Antibiotics were added to the liquid media not before the media had cooled down to about $50^{\circ} \mathrm{C}$ (after autoclaving).

\subsubsection{Frequently used buffers and solutions}

DAPI mounting shield (embedding medium)

$10 \%(\mathrm{v} / \mathrm{v})$ PBS, $90 \%(\mathrm{v} / \mathrm{v})$ glycerol, $1 \mathrm{mg} / \mathrm{ml}$ phenylenediamin, $1 \mu \mathrm{g} / \mathrm{ml}$ 4,6-diamino-2phenylindol (DAPI)

$\underline{\operatorname{PBS}(10 \mathrm{x})}$

1.4 M NaCl, 27 mM KCl, 15 mM KH $2 \mathrm{PO}_{4}, 90$ mM Na $2 \mathrm{HPO}_{4}$ (pH 7.4)

$\underline{\text { TBE buffer }(10 \mathrm{x})}$

0.9 M Tris base ( $\mathrm{pH} 8.3$ ), 0.9 M boric acid, 0.025 M EDTA

$\underline{\text { TE buffer }}$

10 mM Tris-HCl (pH 8.0), 1 mM EDTA (pH 8.0)

TELT buffer

50 mM Tris- $\mathrm{HCl}$ (pH 7.5), 62.5 mM EDTA, $2.5 \mathrm{M} \mathrm{LiCl,} 4$ \% (v/v) Triton X-100 


\section{$\underline{\text { Transport buffer }}$}

$20 \mathrm{mM}$ HEPES-KOH ( $\mathrm{pH}$ 7.4), $110 \mathrm{mM}$ potassium acetate, $5 \mathrm{mM}$ magnesium acetate, $0.5 \mathrm{mM}$ EGTA, 2 mM DTT

$\underline{\text { SDS-PAGE loading buffer }(3 \mathrm{x})}$

('Sample buffer'): 150 mM Tris-HCl (pH 6.8), $3 \%$ (w/v) SDS, $3 \%$ (v/v) $\beta$-mercaptoethanol, $30 \%$ (w/v) glycerol, $0.003 \%$ (w/v) bromophenol blue

DNA gel loading buffer $(4 \mathrm{x})$

$40 \%(\mathrm{w} / \mathrm{v})$ sucrose (in water), $0.1 \%(\mathrm{w} / \mathrm{v})$ bromophenol blue, $0.1 \mathrm{M}$ EDTA (pH 7.5)

\subsubsection{Liquid (growth) media and agar plates}

\section{$\underline{\text { Prokaryotes }}$}

Luria-Bertani (LB) Medium:

$1 \%(\mathrm{w} / \mathrm{v})$ bacto-tryptone, $0.5 \%(\mathrm{w} / \mathrm{v})$ bacto-yeast extract, $1 \%(\mathrm{w} / \mathrm{v}) \mathrm{NaCl}, \mathrm{pH} 7.0$

LB agar plates:

$1.5 \%(\mathrm{w} / \mathrm{v})$ bacto-agar in LB Medium (recipe given above)

2YT Medium:

$0.5 \%(\mathrm{w} / \mathrm{v}) \mathrm{NaCl}, 1.6 \%(\mathrm{w} / \mathrm{v})$ bacto-tryptone, $1 \%(\mathrm{w} / \mathrm{v})$ bacto-yeast extract

\section{$\underline{\text { Eukaryotes }}$}

MEM-Medium: 10.4 g/1 MEM-powder, 2.38 g/1 HEPES, 2.2 g/1 NaHCO 3,10 \% FCS

Dulbecco's MEM (DMEM)-Medium: DMEM-powder, 3.8 g/ $1 \mathrm{NaHCO}_{3}, 10 \%$ FCS

Before use, FCS was inactivated for 45 minutes at $56^{\circ} \mathrm{C}$. In general, the mediums were sterilized (filtration) and stored at $4^{\circ} \mathrm{C}$.

\subsubsection{Antibiotics}

\section{$\underline{\text { Prokaryotes }}$}

Ampicillin (Binotal $\left.{ }^{\circledR}\right)$ (Ratiopharm, Ulm):

Kanamycin sulfate (Serva, Heidelberg):

Tetracycline (Sigma, Deisenhofen):
Stock solution: $100 \mathrm{mg} / \mathrm{ml}$ in $\mathrm{H}_{2} \mathrm{O}$

Working concentration: $100 \mu \mathrm{g} / \mathrm{ml}$

Stock solution: $10 \mathrm{mg} / \mathrm{ml}$ in $\mathrm{H}_{2} \mathrm{O}$

Working concentration: $50 \mu \mathrm{g} / \mathrm{ml}$

Stock solution: $5 \mathrm{mg} / \mathrm{ml}$ in ethanol

Working concentration: $12.5 \mu \mathrm{g} / \mathrm{ml}$ 


\section{Eukaryotes}

Gentamycin (PAA, Cölbe) stock solution $10 \mathrm{mg} / \mathrm{ml}$ in $\mathrm{H}_{2} \mathrm{O}$, working concentration $50 \mu \mathrm{g} / \mathrm{ml}$

Penicillin-Streptomycin (Biochrom, Berlin) $(10 \mathrm{mg} / \mathrm{ml})$, working concentration $1 \%$ (v/v)

\subsubsection{Radioactive isotopes}

$\mathrm{L}-\left[{ }^{35} \mathrm{~S}\right]$-methionine (Amersham Biosciences, Little Chalfont, UK)

$10 \mu \mathrm{Ci} / \mathrm{ml}, 1000 \mathrm{Ci} / \mathrm{mmol}$ (specific radioactivity)

\subsubsection{Vectors}

pCS2+MT (Rupp et al., 1994; Turner and Weintraub, 1994)

pGEX4T-1 (Amersham Biosciences, Freiburg)

pET41a, pET21b (Novagen - Merck Biosciences, Bad Soden)

pQE70 (Qiagen, Hilden), p6z70, p2z70 (see also chapter 2.2.2.15)

pSV $\beta$ (Clontech, Heidelberg), pKS10 (Baake et al., 2001b; Schwamborn et al., 1998)

pcDNA3 (Invitrogen, Karlsruhe), pcHA2, EGFP-LCA1-pcDNA3

\subsubsection{Oligonucleotides}

The oligonucleotides used in this study were exclusively synthesized by MWG-Biotech (Ebersberg), and they are listed in the appendix section (see table II).

\subsubsection{Affinity matrices}

GammaBind $^{\mathrm{TM}}$ Plus Sepharose ${ }^{\mathrm{TM}}$ (Amersham Biosciences, Freiburg)

Glutathione Sepharose CL 4B (Amersham Biosciences, Freiburg)

GST Bind Resin (Novagen - Merck Biosciences, Bad Soden)

IgG Sepharose ${ }^{\circledR}$ Fast Flow (Amersham Biosciences, Freiburg)

Nickel-NTA Agarose (Qiagen, Hilden)

\subsubsection{Software}

Windows XP, Microsoft Word, Microsoft Excel, Microsoft PowerPoint, DNA Star, ImageQuant, Freehand, Adobe Photoshop, EndNote, Unicorn, Boxshade, Spot 


\subsubsection{Hardware}

\section{$\underline{\text { Various }}$}

ÄktaPurifier-System (Amersham Biosciences, Freiburg)

Dialysis membranes (Roth, Karlsruhe)

Digital Sonifier (Branson Sonifier, Schwäbisch Gmünd)

Easyject II (Eurogenetec, Liège, Belgium)

Electroporation cuvettes (Peqlab, Erlangen)

Magnetic stirrer IKAMAG® RET (Jalunke \& Kunkel, Staufen)

Mini-gel-chambers (Biometra, Göttingen; workshop)

Photometer ULTROSPEC 3000 (Amersham Biosciences, Freiburg)

Power supply Power Pack P25 (Biometra, Göttingen)

Speed Vac SC 100 (Savant, Holbrook, NY, USA)

Vortex Genie $2^{\mathrm{TM}}$ (Bender \& Hobein, Zürich, Schweiz)

\section{$\underline{\text { Centrifuges }}$}

Biofuge pico (Heraeus, Hanau)

Megafuge 1.0 (Heraeus, Hanau)

Sorvall RC 5B Plus (Kendro, Langenselbold)

Table centrifuge 5415 (Eppendorf, Hamburg)

Varifuge 3.0 R (Heraeus, Hanau)

Microinjection

Microinjector 5242 (Eppendorf, Hamburg)

Needle puller (Leitz, Wetzlar; Science Products, Hofheim)

$\underline{\text { PCR }}$

GeneAmp PCR system 2400 (Perkin Elmer, Freyung)

T3 Thermocycler (Biometra, Göttingen)

\section{Microscope}

Fluorescence microscope Axioskop 20 (Zeiss, Oberkochen)

Inverse microscope Axiovert 25 (Zeiss, Oberkochen)

Stereomicroscope Stemi SV6, Stemi DV4,

\section{$\underline{\text { Cell culture }}$}

Cell-counter Casy Counter (Schärfe System, Reutlingen)

$\mathrm{CO}_{2}$-Inkubator Heraeus BBD 6220 (Kendro, Langenselbold)

Incubator cytoperm 2 (Heraeus, Hanau)

Incubator Modell CO 24 (New Brunswick, Edison, USA) 
Incubator Shaker Modell G 25 (Scientific Co. Inc, New Jersey, USA)

Incubator Typ B5050 (Heraeus, Hanau)

Liquid nitrogen storage container (Air Liquide Kryotechnik, Düsseldorf)

Horizontal laminar flow hood HERAsafe Typ 18/2 (Heraeus, Hanau)

\section{$\underline{\text { Additional equipment }}$}

Single use (one way) articles as well as technical devices (not listed above) were purchased from the following companies: Eppendorf (Hamburg), Falcon (Heidelberg), Schütt (Göttingen), Greiner (Frickenhausen), Heinemann (Duderstadt), Qiagen (Hilden), Sarstedt (Langenhagen), Siemens (Hannover). 


\subsection{Methods}

Most of the methods used in this study have been performed literally according to handbooks, in other words, the majority of the methods and the theory behind are extensively described in Sambrook et al. (Sambrook et al., 1989) as well as in the 'QIAexpressionist' (Qiagen handbook).

\subsubsection{Cell culture}

\subsubsection{Prokaryotic cells}

\subsection{Bacterial cell maintenance (and growth)}

E. coli cells are the universal working horses of molecular biologists and are mainly used to propagate plasmid DNA and to produce desired gene products (proteins). To recover bacteria, the culture was either streaked onto the surface of an LB agar plate or directly added to the liquid media containing the suitable antibiotics. The LB agar plate or the liquid media was then incubated at $37^{\circ} \mathrm{C}$ overnight. In the first case, next day appropriate liquid medium was inoculated with a single colony (or smear of several colonies) and incubated again at $37^{\circ} \mathrm{C}$ for $12-14$ hours. The resultant pre-culture was then used to set up a main culture. To inoculate the main culture not more than $1 / 10$ of the final volume were used from the pre-culture. Again, also the main culture was incubated at $37^{\circ} \mathrm{C}$ until the desired optical density $\left(\mathrm{OD}_{600}\right)$ was reached for induction.

Certain gene products, however, are considered to be toxic since they already negatively affect the host cell's growth rate at low concentrations. In this case, maintenance of cells can be rather complicated because the desired cells are likely to be outgrown by cells that do not express the toxic protein (mutants). Thus, the level of basal transcription should be repressed as much as possible to reduce the effects of protein toxicity on cell growth prior to induction.

\subsection{Counting bacteria: measuring the optical density}

Bacteria are too small to be counted through direct visualization. They can be counted, however, assuming that each bacterium is capable of forming an individual colony, and the mass of a bacterial suspension can be deduced from its optical density (OD). Further, the OD of a bacterial suspension also indicates in which growth phase the cells are. Measurement was done at $600 \mathrm{~nm}$ with a photometer (Amersham Biosciences) using single use cuvettes with a thickness (width) of $1 \mathrm{~cm}$. 


\subsection{Storage media}

In general, bacteria can be stored for quite a long time (indefinitely) in cultures containing glycerol. For that, to $0.5 \mathrm{ml}$ of the respective bacterial culture, $0.5 \mathrm{ml}$ of sterile glycerol was added. Afterwards the culture was vortexed and frozen either at $-20^{\circ} \mathrm{C}$ (working culture) or at $-70^{\circ} \mathrm{C}$ (long-term storage). To recover the bacteria, the culture was streaked onto the surface of an LB agar plate or directly added to the liquid media containing the appropriate antibiotics.

\subsection{Preparation of competent cells}

Bacteria have to be made 'competent' to be able to take up naked DNA. Treatment with a calcium chloride solution in the early log phase of growth prepares bacteria cells in that way. In more detail, the bacterial cell membrane is permeable to chloride ions, but is non-permeable to calcium ions. As the chloride ions enter the cell, water molecules accompany the charged particle. This influx of water causes the cells to swell which is necessary for the subsequent uptake of DNA.

For preparation of competent bacterial cells, a glycerol cell culture stock of the respective E. coli strain was thawed and added to $50 \mathrm{ml}$ of liquid media. This culture then was incubated in a shaker at $37^{\circ} \mathrm{C}$ until an $\mathrm{OD}_{600}$ of 0.4 was reached. The cells were pelleted by centrifugation, resuspended in $10 \mathrm{ml} 0.1 \mathrm{M}$ calcium chloride (sterile), and then incubated on ice for up to 4 hours. After another centrifugation step, the resulting cell pellet was resuspended in $2 \mathrm{ml} 0.1 \mathrm{M}$ calcium chloride again to yield the final competent cell suspension. Glycerol was added to a final concentration of $10 \%$ and aliquots of the competent cells were immediately frozen in liquid nitrogen and stored until use at $-80^{\circ} \mathrm{C}$ (for several months).

\subsection{Bacterial cell transformation}

Bacterial transformation is the process by which bacterial cells take up naked DNA molecules. The mechanism of this uptake is not well understood. However, it is known that calcium chloride treatment combined with a subsequent heat shock is sufficient for the uptake of DNA. Briefly, for DNA transformation, either the entire DNA ligation reaction or about $50 \mathrm{ng}$ of plasmid DNA (in case of a re-transformation) was added to an aliquot of competent cells (pre-thawed on ice). After mixing gently the cells were further incubated on ice for 30 minutes. The mixture was heat-shocked $\left(42^{\circ} \mathrm{C}\right)$ briefly in a water bath for about 2 minutes and then kept on ice for another 5 minutes. To help bacterial cells to recover from the heat shock treatment, cells were incubated with non-selective growth media for up to 2 hours at $37^{\circ} \mathrm{C}$. After recovery, the bacterial 
suspension was plated out onto the surface of an antibiotic-containing agar plate. Once the fluid was taken up by the agar medium, plates were inverted and incubated overnight at $37^{\circ} \mathrm{C}$.

Transformants of previous ligations were generally screened for correct insertion of the coding fragment by restriction enzyme digestion analysis of the plasmid DNA (see chapter 2.2.2.2).

\subsection{Antibiotic selection}

Plasmids used for the cloning and manipulation of DNA have been engineered to harbor the genes for antibiotic resistance. Thus, if the bacterial transformation was plated onto media containing a certain antibiotic (such as ampicillin) only bacteria which possessed the appropriate plasmid DNA were able to metabolize the antibiotic and formed colonies. In this way, bacterial cells containing plasmid DNA were selected.

\subsubsection{Eukaryotic cells}

\subsection{Maintenance}

The nutrient requirements of eukaryotic cultures are much more complex than the simple broth cultures of $E$. coli. In addition eukaryotes require supplemental sources of materials, typically in the form of blood serum. For this purpose, fetal calf serum (FCS) is used in general. Besides providing growth factors, one of the obvious advantages of FCS is the fetal nature of the serum which limits the presence of antibodies that otherwise might negatively effect cell growth.

For this study, HeLa cells (DZMZ) were grown in modified Eagle's medium (MEM, Biochrom) containing $10 \%(\mathrm{v} / \mathrm{v})$ FCS (PAA) and were maintained in a humidified incubator with $5 \% \mathrm{CO}_{2}$ atmosphere at $37^{\circ} \mathrm{C}$. Cells growing in culture show a distinct growth pattern. Initially they go through a quiescent phase (also often called lag phase) during which there is little or no increase in the cell number. Then cells will go into exponential growth (log phase) where they have the highest metabolic activity, and growth will continue as long as there is sufficient nutrient to support the increasing cell number. Eventually, the cells will enter into stationary phase (plateau phase) where the number of cells remains constant. At this point of confluent population cells have to be subcultured (passaged) or they will eventually die.

\subsection{Passaging/Subculturing/Trypsinizing}

In case of the HeLa cells, confluent cultures were split about 1:5 every three days which could be repeated up to 25 times for a single culture. However, adherent cultures such as HeLa cells need to be removed from the growing surface to be passaged or harvested. A quick and easy method to remove the cells from the growth surface is physical disruption by using a rubber spatula. The 
mechanical removal of cells, however, results in a significant cell death and was therefore only used when cells were harvested for preparing extracts. For subculturing, the proteolytic enzyme trypsin combined with EDTA was used to detach the HeLa cells from the growth surface. Briefly, the medium (MEM) was removed and the culture flask was rinsed with PBS (140 mM $\mathrm{NaCl}, 2.7 \mathrm{mM} \mathrm{KCl}, 1.5 \mathrm{mM} \mathrm{KH}_{2} \mathrm{PO}_{4}, 9.0 \mathrm{mM} \mathrm{Na} 2 \mathrm{HPO}_{4}, \mathrm{pH}$ 7.4) once. Then PBS was removed and replaced with a minimal volume of trypsin/EDTA $(0.05 \%(\mathrm{w} / \mathrm{v}) \operatorname{trypsin}, 0.02 \%(\mathrm{w} / \mathrm{v})$ EDTA in PBS). The following incubation at $37^{\circ} \mathrm{C}$ for 2 minutes allowed the proteolytic reaction to proceed. Once the cells were detached (additionally enforced by shaking) proteolysis was terminated by addition of 5 volumes of complete MEM (FCS inactivates trypsin), and the cell suspension was transferred to a $50 \mathrm{ml}$ conical (falcon) tube. Finally, the cell suspension was vigorously pipetted up and down, pelleted at $1000 \mathrm{~g}$ for 5 minutes, the supernatant (trypsin/media mixture) was removed, the cell pellet resuspended in fresh media, and then plated at the appropriate density. In case of subsequent cell culture experiments such as transfections and nuclear import assays the cells were re-plated at a density of $1 \times 10^{5}$ cells $/ \mathrm{ml}$ of MEM in wells of a tissue culture plate containing glass coverslips, 24 hours prior to the experiments.

\subsection{Preservation and storage}

Liquid nitrogen $\left(\mathrm{N}_{2}\right)$ is used to preserve and to store cultured cells because the growth of ice crystals is retarded below $-130^{\circ} \mathrm{C}$. Damage by ice crystals, however, is only one reason (others include for instance dehydration and changes in $\mathrm{pH}$ ) why freezing can be and to a certain degree is lethal to cells. To minimize the effects of freezing the cryoprotective agent DMSO (glycerol works equally well) which lowers the freezing point was added to the cell suspension. The cells were cooled slowly from room temperature to $-80^{\circ} \mathrm{C}$ allowing the water to move out of the cells before it freezes (to avoid cell damage). Typically, healthy $3 \times 10^{6}$ cells per $\mathrm{ml}$ freezing medium (20\% FCS; $10 \%$ DMSO in MEM) were used for storage and cells were frozen overnight at $-80^{\circ} \mathrm{C}$ (cryo-tube was thereby wrapped in cellulose). To maximize recovery of the cells when thawed, the cells were warmed very quickly by placing the tube directly from the liquid nitrogen container into a $37^{\circ} \mathrm{C}$ water bath. To avoid contamination the cryo-tube was also placed into methanol. When all the ice crystals were melted, the cells were immediately diluted into prewarmed medium (MEM). Once the cells were attached to the growing surface the medium was changed to remove the traces of DMSO. 


\subsection{Transient transfection of eukaryotes}

In this study two different methods were used to introduce recombinant vectors/plasmid DNAs into cultured mammalian cells $(\mathrm{HeLa})$, a process called transfection. Initially, electroporation was used to transfect HeLa cells, however, soon it became obvious that transfection with liposome-like vesicles was much more efficient; hence, it became the method of choice. $1 \times 10^{5}$ HeLa cells/ml MEM were seeded on $10 \mathrm{~mm}$ glass coverslips 24 hours prior to chemical transfection.

\subsection{Electroporation}

Electroporation is a method where a brief, high-voltage electric impulse is applied to a cell that creates nanometer-sized pores in the plasma membrane through which DNA can be taken up directly. Electroporation was performed with the Easyject system (Eurogenetec). In general, a total of $8 \times 10^{5}$ cells and $25 \mathrm{mg}$ plasmid DNA were electroporated in special electroporation cuvettes in a total volume of approximately $1 \mathrm{ml}$ MEM. Electroporation parameters were: $240 \mathrm{~V}$, $1350 \mathrm{mF}, 156 \Omega$, and pulse time $211 \mathrm{~ms}$. Subsequently, the transfected HeLa cells were seeded in about $4 \mathrm{ml}$ MEM in 6-well tissue culture plates and were grown on glass coverslips. After 24 hours or 48 hours the subcellular localization of the transport substrate (translated target gene) was detected by either direct or indirect immunofluorescence.

\subsection{Chemical transfection (lipofection)}

Chemical transfections with artificial membrane vesicles (liposomes) are based on the observation that DNA that is coated with a synthetic cationic lipid can be introduced into cells by fusion. Besides the much better efficiency compared to the electroporation also much less DNA was necessary to successfully transfect HeLa cells. Typically, chemical transfections were done with about 400 ng DNA (only 1/60 of the amount used for electroporation) of each construct using the Effectene ${ }^{\mathrm{TM}}$ Transfection Reagent (Qiagen) according to manufacturer's instructions. Briefly, in the first step of Effectene-DNA complex formation, the DNA was condensed by interaction with the Enhancer in a defined buffer system. Effectene Reagent was then added to the condensed DNA to produce condensed Effectene-DNA complexes. The Effectene-DNA complexes were mixed with MEM and directly added to the HeLa cells.

Note, since the Effectene Reagent forms micelle structures that show no size or batch variation, as found with pre-formulated liposome reagents, excellent reproducibility of transfection complex formation was ensured. 


\subsubsection{Standard methodes involving DNA}

\subsubsection{Quantitation of DNA}

To quantitate DNA (or RNA) two types of methods were used. Mostly, the amount of the nucleic acid was determined by spectrophotometric measurement of the amount of ultraviolet irradiation absorbed by the bases. To only estimate the amount of DNA the intensity of fluorescence emitted by ethidium bromide was used (see also chapter 2.2.2.4). In more detail, for quantitating the amount of DNA by spectrophotometric measurement readings were taken at two wavelengths, $260 \mathrm{~nm}$ and $280 \mathrm{~nm}$. The measurement at $260 \mathrm{~nm}$ allowed the calculation of the concentration of DNA in the sample. In case of:

dsDNA: $\mathrm{OD}_{260}=1$ corresponds to approximately $50 \mu \mathrm{g} / \mathrm{ml}$

ssDNA: $\mathrm{OD}_{260}=1$ corresponds to approximately $40 \mu \mathrm{g} / \mathrm{ml}$

The ratio between the readings at $260 \mathrm{~nm}$ and $280 \mathrm{~nm}\left(\mathrm{OD}_{260} / \mathrm{OD}_{280}\right)$ was used to estimate the purity of DNA. Pure preparations of DNA have a $\left(\mathrm{OD}_{260} / \mathrm{OD}_{280}\right)$ ratio of 1.8 .

\subsubsection{Restriction enzyme digestion}

Restriction enzymes bind specifically to double-stranded (ds) DNA at specific sites within a particular sequence (recognition sequence). Usually, the recognition sequence consists of six nucleotides and displays a twofold symmetry (dyad symmetry). However, the location of the cleavage sites within the recognition sequence differs from enzyme to enzyme. Thus, cleaving both strands at the axis of symmetry generates blunt end fragments of DNA, whereas the cleaving on opposite sides of the axis of symmetry creates so called sticky end DNA fragments. Restriction digestions were performed by incubating dsDNA with an appropriate amount of restriction enzyme in its respective buffer as recommended by the supplier, and at the optimal temperature for that specific enzyme. Typical digestion included $1 \mathrm{U}$ of enzyme per $1 \mu \mathrm{g}$ of DNA. To ensure complete digestion, the reactions were usually incubated for 3 hours at the optimal temperature for enzyme activity (in general $37^{\circ} \mathrm{C}$ ). If necessary, more than one enzyme was included in the digest. However, double digestions were only possible if both enzymes were active in the same buffer and at the same incubation temperature.

\subsubsection{3 'Fill in' reactions governed by Klenow Polymerase and T4 DNA Polymerase}

Restriction enzyme digestion as described above often leads to the formation of sticky end fragments of DNA (either recessed or protruding 3' termini). However, for certain cloning purposes it is sometimes necessary to blunt (polish) these ends, which is generally achieved using Klenow DNA polymerase and/or T4 DNA polymerase. Both enzymes possess a 5' $\rightarrow 3$ ' 
polymerase activity and a 3 ' $\rightarrow$ ' ' exonuclease activity. However, the Klenow DNA polymerase is used to fill recessed 3' termini whereas the potent single-stranded 3' $\rightarrow$ ' ' exonuclease activity of T4 DNA polymerase is used to remove protruding 3' termini (degradation of 3' overhangs). The exonuclease activity of T4 DNA polymerase is more than 200 times that of the Klenow DNA polymerase.

In this study the 3 ' $\rightarrow$ ' exonuclease activity of T4 DNA polymerase (New England Biolabs) was used to blunt DNA fragments. A typical reaction was performed at $12^{\circ} \mathrm{C}$ for 20 minutes with 1-3 U of T4 DNA Polymerase per $1 \mu \mathrm{g}$ DNA fragment, the appropriate buffer, $50 \mu \mathrm{g} / \mathrm{ml} \mathrm{BSA}$ and $100 \mu \mathrm{M}$ dNTPs (final concentration). The reaction was carried out in the presence of high concentrations of dNTPs and at $12^{\circ} \mathrm{C}$ to balance further degradation of the dsDNA (template) by synthesis (at $12^{\circ} \mathrm{C}$ the ratio of polymerase activity to exonuclease activity is maximal).

\subsubsection{Agarose gel electrophoresis}

Agarose gel electrophoresis was employed to separate, identify, and purify DNA fragments. For example, it was used to determine the yield and purity of a DNA isolation or PCR reaction, to check the progression of a restriction enzyme digestion, and to size fractionated DNA molecules, which often were recovered from the gel and used for subsequent cloning purposes.

In general, $1 \%$ agarose in TBE buffer [90 mM Tris base (pH 8.3), $90 \mathrm{mM}$ boric acid, $2.5 \mathrm{mM}$ EDTA] was used; however, in cases where the size of the DNA molecules was smaller than $1 \mathrm{~kb}$, gels containing up to $3 \%$ agarose were prepared, depending on the expected fragment sizes. To load the sample wells the respective DNA was mixed with 1/4 volume of gel loading buffer (40\% (w/v) sucrose in water, $0.1 \%(\mathrm{w} / \mathrm{v})$ bromophenol blue, $0.1 \mathrm{M}$ EDTA). The gel loading buffer thereby served the purpose of increasing the density of the sample and contained a tracking dye (bromophenol blue) which also simplified the loading process. In addition, appropriate size markers were co-electrophoresed with the DNA samples. Usually, agarose gel electrophoresis was performed at $100 \mathrm{~mA}$ for 2 hours at room temperature, but of course the conditions chosen depended on the desired separation. DNA was visualized with the fluorescent dye ethidium bromide (Sharp et al., 1973). Ethidium bromide contains a planar group that inserts between the stacked bases of DNA. The fixed position of this group dramatically increases the fluorescent yield compared to that of unbound dye. Hence, the DNA gels were stained for 15 minutes in an ethidium bromide solution ( $3 \mu \mathrm{g}$ ethidium bromide in 11 water) and the DNA separation pattern was visualized under UV light (radiation at $302 \mathrm{~nm}$ ). In case where the DNA fragments should be recovered and subsequently be purified, the DNA was visualized under UV light with longer wave length (radiation at $366 \mathrm{~nm}$ ) to avoid damage of the DNA sample. 


\subsubsection{Elution of DNA fragments from agarose (QIAEX II)}

DNA fragments were eluted and purified from agarose gels using the QIAEX ${ }^{\circledR}$ II Agarose Gel Extraction Kit (Qiagen) according to the instructions given by the manufacturer. Briefly, the band of interest was excised with a sterile razor blade, placed in a microcentrifuge tube, three times the volume QX1 solution was added, and then the gel was melted at $50^{\circ} \mathrm{C}$. Once the gel was completely molten about $20 \mu 1$ of glass beads (matrix) were added to the solution and further incubated for at least 10 minutes at $50^{\circ} \mathrm{C}$. During the incubation time DNA binds more or less efficiently to the glass beads. Afterwards, the solution containing the glass beads was centrifuged for 1 minute at $13000 \mathrm{rpm}$. Finally, the beads were washed three times (firstly with QX1, then twice with buffer PE), air dried and subsequently eluted with $30 \mu 1$ Tris-HCl buffer (pH 8.5).

\subsubsection{Polymerase chain reaction (PCR)}

The polymerase chain reaction (PCR) was used to amplify defined DNA segments (generally, segments that lie between two regions of a known sequence). For that purpose, two different thermostable DNA polymerases (RedTaq ${ }^{\mathrm{TM}}$, Sigma and Vent $_{\mathrm{R}}{ }^{\circledR}$, New England BioLabs) which both have a 5 ' $\rightarrow 3$ ' polymerization-dependent exonuclease activity were used. Amplification was accomplished by using two oligonucleotide (oligo) primers complementary to the sequences that flanked the region of interest. Typically these oligo primers contained additional restriction enzyme recognition sequences located at the 5' end which allowed subsequent (sub)cloning of the purified PCR products. The PCR was performed in a thermocycler (Biometra, Perkin Elmer) and usually comprised a total volume of $100 \mu \mathrm{l}$ with about $100 \mathrm{ng}$ of template DNA, 100 pmol of both the forward and reverse primers, $5 \mu 1$ of $2 \mathrm{mM}$ dNTPs, 1/100 (v/v) DMSO, $2 \mathrm{U}$ of Vent polymerase (Biolabs) and 1/10 (v/v) of the Vent polymerase buffer (Biolabs). An ordinary cycling protocol consisted of 30 cycles of three temperatures: strand denaturation for 1 minute at $95^{\circ} \mathrm{C}$, primer annealing for 1 minute at $55^{\circ} \mathrm{C}$, and primer extension (elongation) at $72^{\circ} \mathrm{C}$ for 1 minute (the elongation time, however, depends on the size of the expected DNA fragment; usually 1 minute per $1000 \mathrm{bp}$ ). The last cycle included a final elongation step of 7 minutes at $72^{\circ} \mathrm{C}$. After PCR, aliquots of the mixture $(10 \mu \mathrm{l})$ were loaded onto an agarose gel and were electrophoresed to detect and to verify the amplified product. After verification the remaining PCR product was purified with the E.Z.N.A Cycle-Pure Kit (Peqlab) according to the manufacturer's instructions and then digested with the appropriate restriction enzymes (see chapter 2.2.2.2). 


\subsubsection{Alkaline phosphatase treatment (dephosphorylation of cut vectors)}

Alkaline phosphatase (either bacterial or calf intestinal) catalyzes the removal of 5'-phosphate residues from DNA and other substrates such as RNA, and dNTPs. Here, calf intestinal alkaline phosphatase (CIP) was used to remove 5'-phosphates from fragments of DNA (e.g. linearized vectors) to prevent self-ligation. In general, CIP has to be completely removed or inactivated if subsequent ligations are supposed to work efficiently; however, in most of the cases ligations worked out even in the presence of active CIP. To dephosphorylate, vector DNA was incubated with $10 \mathrm{U}$ CIP for 30 minutes at $37^{\circ} \mathrm{C}$. In case of heat inactivation of CIP the reaction mixture was kept at $75^{\circ} \mathrm{C}$ for 10 minutes in the presence of $5 \mathrm{mM}$ EDTA ( $\mathrm{pH}$ 8.0). Depending on the size of the DNA molecule that was previously cut out of the vector plasmid, the entire reaction mixture was purified using either the E.Z.N.A Cycle-Pure Kit (Peqlab) according to the manufacturer's instructions (spin-column purification, cut out DNA fragments smaller than $50 \mathrm{bp}$ ) or the agarose gel electrophoresis (DNA fragments larger than $50 \mathrm{bp}$; see chapter 2.2.2.4).

\subsubsection{DNA ligation}

Phosphodiester bonds between adjacent 3'-hydroxyl and 5'-phosphate termini in DNA can be joined (ligated). T4 DNA ligase catalyzes the formation of these bonds consuming ATP as energy source. Thus, DNA ligase was used to join linearized cloning vector and insert DNA (PCR product) carrying compatible cohesive termini (respectively blunt ends). Typical ligations were performed by incubating about $100 \mathrm{ng}$ of linearized cloning vector with a five times molar excess of insert DNA in the presence of ligase buffer and 0.5 U T4 DNA ligase (New England Biolabs) for 16 hours at $12^{\circ} \mathrm{C}$, respectively for 2 hours at $25^{\circ} \mathrm{C}$. In addition, control ligations were performed in the absence of insert DNA to determine the background clones arising from self-ligation of inefficiently dephosphorylated vector.

\subsubsection{Annealing of single stranded oligonucleotides}

Since only double stranded (ds) DNA fragments are integrated into vector DNA, single stranded (ss) oligonucleotides have to be annealed first. This is of particular interest if the DNA fragments that are supposed to be integrated are very small [e.g. affinity tags such as HA or additional restriction digestion sites or enzyme (thrombin) cleavage sites]. For that purpose, usually $5 \mu 1$ (500 pmol) of both complementary oligonucleotides (which include appropriate overhanging restriction digestion sites for efficient subcloning) were mixed with $40 \mu \mathrm{d}_{2} \mathrm{O}$ (or alternativly annealing buffer: $0.04 \mathrm{M}$ Tris/ $\mathrm{HCl}, \mathrm{pH} 7.6,0.06 \mathrm{M} \mathrm{NaCl}$ ) and heated in a large water bath for 5 minutes at about $95^{\circ} \mathrm{C}$. The water bath was allowed to slowly cool down to room temperature 
which finally led to the annealing/hybridization of the two complementary oligonucleotides. Eventually, $2 \mu \mathrm{l}$ of the $50 \mu \mathrm{l}$ mixture were further used for phosphorylation of the double stranded oligonucleotide (see next chapter).

\subsubsection{Kinase end-labeling of DNA (T4 polynucleotide kinase)}

T4 polynucleotide kinase (T4 PNK) catalyzes the transfer of the $\gamma$-phosphate of ATP to a 5'-hydroxyl terminus of DNA (or RNA). Thus, T4 PNK can be used to phosphorylate doublestranded oligonucleotides that are intended to be ligated in a dephosphorylated vector afterwards. A typical 5'-kinase labeling reaction included $50 \mathrm{pmol}$ double stranded oligonucleotide, $1 \mathrm{mM}$ ATP, $10 \mathrm{U}$ T4 polynucleotide kinase $(10 \mathrm{U} / \mu \mathrm{l})$, and the appropriate kinase buffer (10x PNK buffer: $0.07 \mathrm{M}$ Tris/ $\mathrm{HCl} \mathrm{pH}$ 7.6). The phosphorylation reaction was performed for 30 minutes at $37^{\circ} \mathrm{C}$. After incubation the reaction was usually heat inactivated by incubation at $70^{\circ} \mathrm{C}$ for 10 minutes. Portions of the reaction mixture were then used for the ligation reaction.

\subsubsection{Isolation of plasmid DNA (from single colonies) after transformation}

This so-called TELT method (the name is based on the buffer contents, see below) was generally applied to quickly isolate analytical amounts of plasmid DNA of single clones (colonies) after transformation, followed by restriction enzyme digestion analysis (chapter 2.2.2.2). Briefly, individual clones from a plate were picked with a sterile toothpick and were deposited into small glass tubes containing $3 \mathrm{ml}$ of LB media and the appropriate antibiotics. Toothpicks were kept in the media and the cells were allowed to grow for $12-16$ hours in the $37^{\circ} \mathrm{C}$ shaker/incubator at $200 \mathrm{rpm}$. Then, only $1.5 \mathrm{ml}$ of each culture was used to isolate plasmid DNA. Firstly, cells were collected by centrifugation ( $5000 \mathrm{rpm}$ for 5 minutes), the supernatant was discarded, the pellet was resuspended in $150 \mu 1$ TELT buffer [50 mM Tris-HCl, pH 7.5; 62.5 mM EDTA, $2.5 \mathrm{M}$ LiCl, $0.4 \%(\mathrm{w} / \mathrm{v})$ Triton X-100], $15 \mu \mathrm{l}$ lysozyme $\left(10 \mathrm{mg} / \mathrm{ml}\right.$ in $\left.\mathrm{dH}_{2} \mathrm{O}\right)$ were added and subsequently boiled for 2 minutes at $95^{\circ} \mathrm{C}$ (denaturing of bacterial proteins). For efficient precipitation the cells were cooled on ice for 5 minutes and then centrifuged at $14000 \mathrm{rpm}$ for 10 minutes at $4^{\circ} \mathrm{C}$. The resulting pellet containing denatured proteins and cell debris was removed with a sterile toothpick, and addition of $150 \mu 1$ isopropanol (see also chapter 2.2.2.12) resulted in precipitation of plasmid DNA. Again, the pellet was collected by centrifugation (14000 rpm, 15 minutes, and $4^{\circ} \mathrm{C}$ ), washed with $100 \mu 1$ of $70 \%$ ice-cold ethanol, quickly dried in a Speed Vac centrifuge, and

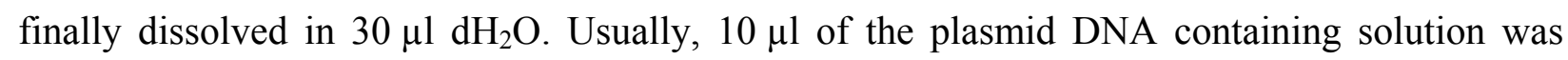
used for subsequent restriction enzyme digestions (to screen for positive clones). In case of expected small digestion products (DNA fragments containing less than $200 \mathrm{bp}$ ) the RNA 
present in the probe had to be additionally digested with $0.5 \mu 1 \mathrm{RNaseA}(10 \mathrm{mg} / \mathrm{ml})$ to be able to identify positive clones comparing digestion patterns after using agarose gel electrophoresis.

\subsubsection{Concentration of DNA by ethanol/isopropanol precipitation}

To precipitate DNA, usually 2.5 - 3 volumes of ethanol were added to the DNA sample in a microcentrifuge tube, which was subsequently placed on ice for at least 10 minutes. In case of isopropanol only $0.7-1$ volume of isopropanol had to be used to precipitate the DNA. Therefore, precipitation using isopropanol is recommended if large volumes of DNA need to be precipitated. To recover the precipitated DNA, the tube was centrifuged, the supernatant was discarded, and the DNA pellet was rinsed with a $70 \%$ ethanol solution. This subsequent wash is applied to remove residual salt and moisture. Afterwards the DNA sample was centrifuged again, the supernatant was discarded, and finally the DNA pellet was dried either on air or in a Speed Vac under vacuum.

\subsubsection{Plasmid purification}

The purification strategy (protocol) was the same for isolation of plasmid DNA in the range from analytical amounts to large scale quantities. Thus, only the aliquots of antibiotic containing liquid media inoculated with the plasmid-containing cell colonies varied (10 $\mathrm{ml}$ to $150 \mathrm{ml})$. Bacteria were incubated in a $37^{\circ} \mathrm{C}$ shaker for $12-16$ hours and the plasmid containing cells were subsequently collected by centrifugation for 10 minutes at $6000 \mathrm{rpm}$ (GSA rotor, Sorvall ${ }^{\circledR}$ ). The actual purification of plasmid DNA was then done using differently sized Qiagen purification kits (mini, midi, maxi) according to the manufacturer's instructions (Qiagen plasmid purification handbooks). Independent of the kit size, the plasmid purification was always based on a modified alkaline lysis, followed by binding of plasmid DNA to anion-exchange resin (QIAGEN-tips) under appropriate low-salt and $\mathrm{pH}$ conditions. Using these columns in combination with medium-salt wash steps guaranteed that RNA, proteins, dyes, and other lowmolecular-weight impurities were efficiently removed. The plasmid DNA was eluted in a highsalt buffer and then concentrated and desalted by isopropanol precipitation (see also chapter 2.2.2.12). Finally, the isolated/purified plasmid DNA was dissolved in either $\mathrm{dH}_{2} \mathrm{O}$ or TE buffer (10 mM Tris-HCl, $\mathrm{pH} 8.0 ; 1 \mathrm{mM}$ EDTA) and stored at $4^{\circ} \mathrm{C}$. 


\subsubsection{DNA sequencing}

For DNA sequencing the Sanger dideoxy method was used (Sanger et al., 1977). This method takes advantage of the requirement for a free 3' hydroxyl group to form the necessary phosphodiester bridge between two nucleotides during DNA polymerization.

Briefly, DNA sequencing reactions based on this method are performed on any given template DNA using a specific primer, DNA polymerase, and a mixture of 2'-deoxynucleotide 5'-triphosphates (dNTPs) and 2',3'-dideoxynucleotide 5'-triphospates (ddNTPs) as substrates. ddNTPs differ from dNTPs by having a hydrogen atom attached to the 3' carbon rather than a hydroxyl group. When dNTPs are incorporated into a growing DNA strand, chain elongation proceeds normally. However, when a ddNTP is incorporated the process of chain elongation is terminated because of the absence of the 3' hydroxyl group. Thus, DNA polymerization products are of different lengths depending on when a ddNTP was integrated into the growing strand. Theoretically, there should be a ddNTP incorporated at least once at each of the base positions for any particular DNA template. The four ddNTPs used (part of the Big Dye ${ }^{\mathrm{TM}}$ Terminator Sequencing Kit, Applied Biosystems) for DNA sequencing were tagged with four different fluorescent dyes so that the sequencing products could be detected subsequently by laser scanning in an ABI sequencer (model 373A, Applied Biosystems). Prior to that, however, the pool of fluorescently tagged DNA fragments had to be separated by size which was achieved by capillary electrophoresis. The high resolution of this electrophoresis method easily allows quick detection of differences in size resulting from a single nucleotide.

Typically, for a sequence reaction $200 \mathrm{ng}$ DNA, 100 pmol primer, $1 \mu 1$ sequence buffer $(10 \times)$, and 1-3 $\mu$ l Dye Terminator Ready Mix (Applied Biosystems) were used and $\mathrm{dH}_{2} \mathrm{O}$ was added to a final volume of $10 \mu \mathrm{l}$. The PCR protocol consisted of 25 cycles of three temperatures: strand denaturation for 30 seconds at $95^{\circ} \mathrm{C}$, primer annealing for 15 seconds at $50^{\circ} \mathrm{C}$, and chain elongation for 4 minutes at $60^{\circ} \mathrm{C}$. To purify the sequence products (fluorescently labeled DNA fragments), $1 \mu 13 \mathrm{M}$ sodium acetate, $1 \mu 1125 \mathrm{mM}$ EDTA, and $50 \mu 1$ ethanol were added to the PCR mixture to precipitate the DNA. After centrifugation $\left(15\right.$ minutes, $13000 \mathrm{rpm}$ at $\left.4^{\circ} \mathrm{C}\right)$ the supernatant was carefully removed and the DNA was washed with $70 \mu 1$ of $70 \%$ ethanol. Afterwards the DNA sample was centrifuged again, the supernatant was discarded, and the DNA pellet was dried for 5 minutes in the Speed Vac, and finally dissolved in $25 \mu 1 \mathrm{H}_{2} \mathrm{O}$ (HPLC quality). The subsequent analysis (sequence run) was done by Andreas Nolte and Marco Winkler (Department of Developmental Biochemistry, University of Göttingen) using the above mentioned ABI sequencer (Applied Biosystems). 


\subsubsection{Expression constructs}

The coding regions of the respective proteins were first amplified from plasmid DNA (the cDNA of the NF-Y subunits has been a kind gift from Roberto Mantovani, Dipartimento di Biologia Animale, Università di Modena e Reggio Emilia, Modena, Italy) using specific primer pairs with appropriate restriction sites (for a complete list of expression constructs, see appendix table II).

The various coding regions for the GST-fusion proteins were cloned as follows: murine NF-YA and mutant NF-YA (developed by site-directed mutagenesis (next chapter) and characterized in Fig.8A) as EcoRI-XhoI fragments into the EcoRI-XhoI sites of pGEX4T-1 (Amersham Biosciences; N-GST-tagged; pJK95), human NF-YB as a BamHI-SmaI fragment into the BamHI-SmaI sites of pGEX4T-1 (N-GST-tagged; pJK96); human NF-YC as a SpeI-SacI fragment into the SpeI-SacI sites of pET41a (Novagen; N-GST-tagged; pJK47), human H2A/d as an EcoRI-NotI fragment into the EcoRI-NotI sites of pET41a (N-GST-tagged; pJK61); human $\mathrm{H} 2 \mathrm{~B} / \mathrm{d}$ as an EcoRI-NotI fragment into the EcoRI-NotI sites of pET41a (N-GST-tagged; pJK63), and as a BamHI-SmaI fragment into the BamHI-SmaI sites of pGEX4T-1 (N-GST-tagged; pJK70).

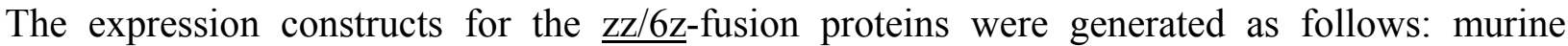
NF-YA as an EcoRV-SpeI fragment into the EcoRV-SpeI sites of p6z70 (pJK35). p6z70 is a modified pQE70 (Qiagen) vector in which 6 z-tags (IgG-binding domain from Staphylococcus aureus protein A) were inserted at the $\mathrm{N}$-terminus in addition to the $\mathrm{C}$-terminal His-tag. Human NF-YB as a SmaI-NheI fragment from the corresponding $\mathrm{pKS}$ vector of the transfection system (described in Schwamborn et al., 1998) into the EcoRV-SpeI sites of p2z70 (modified pQE70 in which 2 z-tags were inserted at the N-terminus in addition to the C-terminal His-tag; pJK1), human $\mathrm{H} 2 \mathrm{~A} / \mathrm{d}, \mathrm{H} 2 \mathrm{~B} / \mathrm{d}, \mathrm{H} 3 / \mathrm{k}$, and $\mathrm{H} 4 / \mathrm{j}$ (respectively fragments of these four core histones) as a SmaI-NheI fragment from the corresponding pKS vector into the EcoRV-SpeI sites of p2z70 (N-2z-tagged) and p6z70 (N-6z-tagged). It is important to note that the $\mathrm{z}$ domains alone neither bind to any of the transport receptors nor get imported by them.

The expression construct for the NF-YA-His-fusion protein was generated as follows: murine NF-YA as NdeI-SacI fragment into the NdeI-SacI sites of pJK45 (C-His-tagged construct, pJK45 is a modified pET21b in which the Pst I-NruI fragment of pACYC177 was inserted into the PstI-PshAI digested pET21b). 
The expression constructs for $\underline{6 m y c-f u s i o n}$ proteins were generated as follows: murine NF-YA (pJK143), NF-YA $\Delta 262-317$ (pJK154), the NF-YA point mutant KRR276-278NLG/RRK294296LGQ (pJK155, see also Fig.8A for more detail), human NF-YB (pJK19, construct pJK163 contains additional $6 \mathrm{z}$ tags at the N-terminus), and human NF-YC (pJK20, construct pJK164 contains additional $6 \mathrm{z}$ tags at the N-terminus) as EcoRI-StuI fragments into the EcoRI-StuI sites of pCS2+MT (Rupp et al., 1994; Turner and Weintraub, 1994) containing 6 myc-tags at the N-terminus.

The expression constructs for $\beta$-galactosidase-fusion proteins were generated as follows: the corresponding coding regions or gene fragments of the different NF-Y subunits were cloned via PCR into the modified pSV $\beta$ (Clontech) mammalian expression vector pKS10 (Baake et al., 2001b; Schwamborn et al., 1998) using the unique restriction sites BglII and NheI. Deletion constructs were generated by combined insertion of two PCR amplified gene fragments (PCR products) cloned into the BglII-NheI and NheI-NruI restrictions sites.

Hemagglutinin ( $\underline{\mathrm{HA}})-\mathrm{NF}-\mathrm{Y}$ fusion protein expression constructs were generated as follows: murine NF-YA as an EcoRI-HindIII fragment into the EcoRI-HindIII sites of pcHA2 (modified pcDNA3 (Invitrogen) with a pBKII SK (+/-) multi cloning site (BamHI-XhoI fragment) and Nterminal HA-tag, pJK114), human NF-YB (pJK115) and human NF-YC (pJK116) as BamHI-SmaI fragments into the BamHI-SmaI sites of pcHA2.

Enhanced green fluorescent protein (EGFP)-NF-Y fusion protein expression constructs were generated as follows: murine NF-YA (pJK108), human NF-YB (pJK132) and human NF-YC (pJK133) as XhoI-ApaI fragments into an XhoI-ApaI digested EGFP-LCA1-pcDNA3 (modified pcDNA3 with an EGFP-LCA1 inserted into the EcoRV-ApaI sites).

The expression constructs for the GST-EGFP-Pax6-fusion protein was generated as follows: Xenopus Pax6 as EcoRI-XhoI fragment into the EcoRI-XhoI sites of pGEX-EGFP (modified pGEX4T-1 in which EGFP was inserted as BamHI-EcoRI fragment).

All constructs were verified by DNA sequencing (see chapter 2.2.2.14). 


\subsubsection{Site-directed mutagenesis}

Nucleotide exchanges in pJK103, pJK104, pJK105, pJK106, pJK130 and pJK131 were done by site-directed mutagenesis as described by Ho et al. (Ho et al., 1989). Thereby, only polymerase chain reactions (PCRs, see chapter 2.2.2.6) were used to introduce the desired mutations in the NF-YA sequence. The following strategy was applied: Two independent PCRs were performed to amplify gene fragments carrying the desired mutation in a complementary region (overlapping ends). The mutated gene fragments from the first two reactions were combined in a subsequent 'fusion' reaction in which the overlapping ends (including the mutation) were annealed. This, however, allowed the 3' overlap of each strand to serve as a primer for the 3' extension of the complementary strand catalyzed by the polymerase. The resulting fusion product was amplified further by a third PCR. Note, for the first two PCRs the use of a proofreading polymerase (possessing a $3{ }^{\prime} \rightarrow 5^{\prime}$ proofreading exonuclease activity) was necessary to avoid protruding 3 ' termini (such as created by taq polymerase for instance) which would have caused a wrong reading frame in the subsequent third PCR. However, Vent ${ }_{R}{ }^{\circledR}$ DNA-Polymerase (New England BioLabs) was used for all three PCRs because in addition to creating desired blunt ends also the probability of additional (unwanted) mutations was drastically reduced. Possessing proofreading activity assures that each nucleotide during DNA synthesis is checked and that mismatched nucleotides in the 3 ' to $5^{\prime}$ direction are excised.

Taken together, for site-directed mutagenesis by overlap extension using the PCR according to Ho et al., 1989 four oligodeoxyribonucleotide (oligo) primers are necessary besides the template DNA. Two of the four oligo primers are complementary to each other and carry the desired mutation (incorporated nucleotide changes). The following oligo primer pairs were used to generate the various mutations: 5'-CTGTATGTGAATGCCGCACAGTATCACCGCATC-3' (sense) and 5'-GATGCGGTGATACTGTGCGGCATTCACATACAG-3' (antisense) for pJK103，5'-AAACAGTATCACCGCATCCTTAATCTGGGACAAGCACGGGCTAAGCTAG AG-3' (sense) and 5'-CTCTAGCTTAGCCCGTGCTTGTCCCAGATTAAGGATGCGGTGAT ACTGTTT-3' (antisense) for pJK104, 5'-GAAGGGAAAATCCCAAAGGAACTAGGGCAAT ACCTCCATGAGTCTCGGCATCGG-3' (sense) and 5'-CCGATGCCGAGACTCATGGAGGT ATTGCCCTAGTTCCTTTGGGATTTTCCCTTC-3' (antisense) for pJK105, 5'-CGGCATCGG CACGCCATGGCACTGGCGGGTGGGGAAGGGGGCCGCTTCTTC-3' (sense) 5'-GAAGAA GCGGCCCCCTTCCCCACCCGCCAGTGCCATGGCGTGCCGATGCCG-3' (antisense) for pJK106. In case of pJK130 and pJK131 the same primers were used as for pJK104 and pJK105, respectively as for pJK104 and pJK106. 


\subsubsection{Proteins}

\subsubsection{SDS-PAGE of proteins (separation of proteins under denaturing conditions)}

In sodium dodecyl sulfate (SDS) polyacrylamide gel electrophoresis (PAGE) separations, migration is determined not by intrinsic electric charge of polypeptides but by molecular weight (Weber and Osborn, 1969). SDS is a strongly anionic detergent that denatures proteins literally by wrapping the hydrophobic tail around the polypeptide backbone thereby conferring a net negative charge. For almost all proteins, the amount of SDS bound is proportional to the molecular weight of the polypeptide. Thus, the SDS-polypeptide complexes migrate through polyacrylamide gels in accordance with the size of the polypeptide. Using markers of known molecular weight therefore allows the estimation of the molecular weight of the polypeptide chain. In addition to SDS, reducing agents such as $\beta$-mercaptoethanol (BME, used in this study) or dithiothreitol (DTT) must be added to completely unfold the proteins (reducing agents cleave/reduce any disulfide bond between cysteine residues).

As in most cases, SDS-PAGE was carried out with a discontinuous buffer system using a stacking and resolving gel (Laemmli, 1970). In contrast to the continuous buffer system which uses only one buffer for reservoirs and gel, in the discontinuous buffer system (originally developed by Davis, 1964; Ornstein, 1964) the reservoir buffer has a different $\mathrm{pH}$ and ionic strength than the buffer used to prepare the gels. In more detail, buffers and gels were composed as follows: $\underline{3 \times \text { sample buffer: }} 9 \%(\mathrm{w} / \mathrm{v}) \mathrm{SDS}, 300 \mathrm{mM}$ Tris- $\mathrm{HCl} \mathrm{pH} \mathrm{6.8,} 22.5 \%(\mathrm{w} / \mathrm{v})$ glycerol, $0.1 \%(\mathrm{w} / \mathrm{v})$ bromophenol blue, $10 \%(\mathrm{v} / \mathrm{v}) \beta$-mercaptoethanol (BME); stacking gel: $5 \%(\mathrm{v} / \mathrm{v})$ acrylamide/bisacrylamide (30:0.8), $0.125 \mathrm{M}$ Tris- $\mathrm{HCl} \mathrm{pH}$ 6.8, $0.1 \%(\mathrm{w} / \mathrm{v}) \mathrm{SDS}, 0.15 \%$ (w/v) ammonium persulfate, $0.1 \%(\mathrm{v} / \mathrm{v})$ TEMED; resolving gel: 5\%-15\% (v/v) acrylamide/ bisacrylamide (30:0.8), 0.375 M Tris- $\mathrm{HCl} \mathrm{pH} 8.8,0.1 \%$ (w/v) SDS, 0.15\% (w/v) ammonium

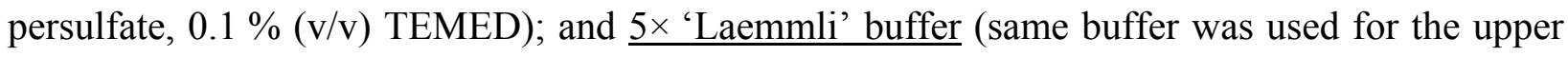
and lower reservoirs): $0.25 \mathrm{M}$ Tris, $1.9 \mathrm{M}$ glycine , $\mathrm{pH} 8.7,0.5 \%(\mathrm{w} / \mathrm{v})$ SDS. When electric current is applied the chloride ions in the sample and stacking gel (leading edge) and the glycine molecules in the 'Laemmli' buffer (trailing edge) form a moving boundary that carries the SDSpolypeptides (sample) across the stacking gel and loads them on the surface of the resolving gel. The higher $\mathrm{pH}$ of the resolving gel leads then to the ionization of glycine molecules which as a result of it no longer trail behind but move right behind the chloride ions through the resolving gel. Hence, SDS-polypeptides suddenly become the trailing ions and separate according to their size by sieving.

The sieving properties of (SDS)-PAGE gels are determined by the size of the pores which themselves are defined by the absolute concentration of acrylamide/bisacrylamide used for 
preparation. Generally, concentrations of acrylamide between $5 \%$ and $15 \%$ allow separations in a range from $250 \mathrm{kDa}$ to $12 \mathrm{kDa}$. Polymerization of acrylamide, however, only occurs in the presence of cross-linking agents such as bisacrylamide (N,N'-methylenebisacrylamide) which as the name indicates cross-links the polymerized acrylamide chain. The polymerization chain reaction occurs in the presence of free radicals, which are usually (also in this study) supplied by ammonium persulfate (radical formation was catalyzed and stabilized by TEMED).

Since the discontinuous buffer system was used, SDS-PAGE was carried out in vertical gels using mini-gel-chambers (Biometra). Usually an electric current of $15 \mathrm{~mA}$ was applied for migration through the stacking gel whereas the electric current was slightly increased to $20 \mathrm{~mA}$ for migration through the resolving gel. As soon as the bromophenol blue color marker had reached the end of the resolving gel (or shortly after the marker had run out of the gel) SDSPAGE was stopped.

Gels could then be fixed and stained with Coomassie Brilliant Blue or silver salts, fluorographed or autoradiographed, or used to establish a western blot. Non-radioactive gels, respectively nonradioactive polypeptides separated by SDS-polyacrylamide gels, were almost always simultaneously fixed with methanol/glacial acetic acid and stained with Coomassie Brilliant Blue (10\% glacial acetic acid, $50 \%$ methanol, $2.5 \mathrm{~g} / 1$ Coomassie Brilliant Blue R250). Gels were subsequently destained by soaking them in the methanol/acetic acid solution (Coomassie Brilliant Blue staining solution omitting the dye). SDS-polyacrylamide gels containing radioactively labeled amino acids $\left({ }^{35} \mathrm{~S}\right.$-labeled) had to be dried before autoradiography (phosphorimaging). To minimize shrinkage and distortion the gel was covered with a foil and sandwiched into Whatman paper before it was dehydrated on the vacuum gel dryer (BioRad) at $70^{\circ} \mathrm{C}$. (Care had to be taken not to remove the gel from the dryer respectively not to turn off the vacuum before the gel is completely dehydrated because it would crack). Prior to phophorimaging (Amersham Biosciences) the gel was exposed on a phosphorimager screen for 1 to 3 days.

\subsubsection{Bradford protein assay}

The Bradford assay is a very fast and accurate method to determine the total protein concentration of a sample (Bradford, 1976). The assay is based on the observation that the absorbance maximum for an acidic solution of Coomassie Brilliant Blue G-250 shifts from $465 \mathrm{~nm}$ to $595 \mathrm{~nm}$ when protein binding occurs. More specifically, protein binding stabilizes the anionic form of the dye through ionic and hydrophobic interactions and eventually causes a visible color change. The more protein is present, the more Coomassie binds, and the more the 
color changes. This of course can be easily detected with a photometer. As in this study, BSA $(1 \mathrm{mg} / \mathrm{ml})$ was generally used as protein standard. BSA standards contained a range of 0 to $20 \mu \mathrm{g} / \mathrm{ml}$ buffer (same buffer as for the protein samples). Also, the protein samples were diluted to an estimated concentration of up to $20 \mu \mathrm{g}$ per $\mathrm{ml}$. In each case $1 \mathrm{ml}$ of Bradford reagent (425 ml dH20, $15 \mathrm{ml} 95 \%$ ethanol, $30 \mathrm{ml} 88 \%$ phosphoric acid, and $30 \mathrm{ml}$ Bradford stock solution) was added and the absorbance (excitation) was measured at $595 \mathrm{~nm} 15$ minutes later. The Bradford stock solution consisted of $100 \mathrm{ml} 95 \%$ ethanol, $200 \mathrm{ml} 88 \%$ phosphoric acid, and $350 \mathrm{mg}$ Coomassie Brilliant Blue G-250.

\subsubsection{Recombinant protein expression and purification}

In general, expression of recombinant proteins is accomplished by starting with a plasmid that encodes the target protein, introducing the plasmid into the appropriate host cell, growing of the host cells, inducing expression, followed by cell lysis and protein purification.

\subsection{Induction by IPTG}

Expression of recombinant proteins was rapidly induced by the addition of isopropylthio- $\beta$-Dgalactoside (IPTG) which bound to the lac repressor protein inactivating it in that way. Once the lac repressor was inactivated, the host cell's RNA polymerase could transcribe the sequences downstream from the promoter. The transcripts produced were then translated into the recombinant protein (for a typical expression and purification, see below, chapter 2.2.3.3.3).

\subsection{Batch or column}

Affinity purification of proteins on nickel-NTA agarose (Qiagen) or glutathione-Sepharose 4B (Amersham Biosciences), respectively GST-Bind resin (Novagen), was in either a batch or a column procedure. The column procedure involved binding of the protein to the appropriate resin (matrix) in solution and subsequent packing of the protein-resin complex into a column for the washing and elution steps. In the batch procedure, the protein-resin complex was not packed into a column, but washing and elution steps occurred in the batch as well.

\subsection{Affinity chromatography (purification of proteins carrying an affinity tag)}

Affinity purification allows the quick and efficient purification of (bacterially expressed) proteins. Thus, the use of tagged proteins is widely used, and among the most commonly taken affinity tags are $6 \times$ His (six histidine residues) and GST (glutathione S-transferase). All recombinant proteins (except NTF2, see also chapter 2.2.3.3.6) used for this study carried at least 
one of these two affinity tags. The $6 \times$ His tag, on one side, is very small and allows affinity purification of recombinant proteins under denatured conditions. The GST-tag, on the other side, is relatively large $(\sim 27 \mathrm{kDa})$ but might increase the expression yield and the solubility of target proteins. While the $6 \times$ His tag facilitates binding to nickel-NTA (nitrilotriacetic acid), GSTtagged proteins efficiently bind to glutathione. NTA occupies four of the six ligand binding sites in the coordination sphere of the nickel ion, leaving two sites free to interact with the $6 \times \mathrm{His}$ tag. Since NTA binds metal ions (in this particular case nickel) more stably than other chelating resins (Hochuli et al., 1987) it can be applied under very different conditions (e.g. under stringent wash conditions). Further, the introduction of a protease cleavage site between the tag and protein of interest allows the removal of the tags when necessary. Usually thrombin cleavage sites are used and thrombin (a serine protease) can subsequently be inhibited by PMSF or removed for instance with the synthetic inhibitor p-amino-benzamidine (e.g. HiTrap ${ }^{\mathrm{TM}}$ Benzamidine FF column). Elution of His-tagged fusion proteins and of GST-tagged fusion proteins from the respective resin (matrix) was with imidazole $(250 \mathrm{mM})$ respectively reduced glutathione $(10 \mathrm{mM})$. As mentioned above, all proteins used in this study carried one of these two affinity tags, hence, the first protein purification step always involved affinity chromatography. Additional purification steps then depended on the obtained protein purity and usually included ion exchange chromatography and gel filtration (size exclusion chromatography).

Typically, a $50 \mathrm{ml}$ culture of the selected clone was grown at $37^{\circ} \mathrm{C}$ overnight. The $50 \mathrm{ml}$ culture was scaled up to $1000 \mathrm{ml}$ and allowed to grow until the OD reached a value of about 1.0 at $600 \mathrm{~nm}$. Then, the culture was induced with isopropyl-ß-D-thiogalactopyranoside (IPTG) at a final concentration of $0.2 \mathrm{mM}$ to $1.0 \mathrm{mM}$ and incubated at $17^{\circ} \mathrm{C}$ to $30^{\circ} \mathrm{C}$ for $2-4$ hours (always depending on the particular protein requirements). Cells were then collected by low speed centrifugation, resuspended in the appropriate buffer (generally containing at least $300 \mathrm{mM} \mathrm{NaCl}$ and a mix of protease inhibitors), frozen and thawed, and lysed by sonication for $2 \times 60$ seconds in an ice-water bath (always with 10 second breaks after 10 seconds of sonication; amplitude $43 \%$ ). The bacterial lysate was cleared by centrifugation at $15000 \mathrm{~g}$ (SS 34 rotor, Sorvall@) for 30 minutes at $4^{\circ} \mathrm{C}$. The resultant supernatant was then allowed to bind for 3 hours at $4^{\circ} \mathrm{C}$ to $500-1000 \mu \mathrm{l}$ of the corresponding affinity matrices (nickel NTA-agarose, glutathioneSepharose). After several repeated washing steps (in case of His-tagged fusion proteins endogenous bacterial proteins with histidine residues that interact with the nickel-NTA could be washed out of the matrix with stringent conditions achieved by adding imidazole at a $10-50 \mathrm{mM}$ concentration) recombinant fusion proteins were eluted with imidazole or reduced glutathione, 
respectively. Adding of imidazole in a concentration of $100-250 \mathrm{mM}$ resulted in dissociation of His-tagged proteins because they could no longer compete for binding sites on the nickel-NTA matrix. Similarly, the addition of $10 \mathrm{mM}$ reduced glutathione caused dissociation of GST-tagged proteins because the binding of immobilized glutathione (resin) to GST was competed by the unbound glutathione.

\subsection{Denaturing conditions}

In many cases, especially at high expression levels, recombinant proteins expressed in E. coli aggregate and form insoluble inclusion bodies. Inclusion bodies strongly limit the utility of standard purification procedures which rely on the protein's native soluble form (such as GST affinity chromatography). However, since the interaction between nickel-NTA and the His tag of recombinant proteins does not depend on tertiary structure, His-tagged fusion proteins can also be purified under denaturing conditions. Thus, nickel-NTA affinity chromatography is not affected by problems arising from protein insolubility. To solubilize proteins expressed in inclusion bodies, detergents or denaturants such as $8 \mathrm{M}$ urea or $6 \mathrm{M} \mathrm{GuHCl}$ are generally used before the actual purification steps are initiated. In this study $6-8 \mathrm{mM}$ urea was applied to solubilize His-tagged proteins (particularly core histone fusion proteins) expressed in inclusion bodies.

Briefly, cells collected by low speed centrifugation or insoluble cell pellets resulting from purification/resuspension under native conditions were resuspended in a urea buffer (6 M urea, $1 \mathrm{M} \mathrm{NaCl}, 50 \mathrm{mM}$ Tris-HCl pH 7.0, $10 \mathrm{mM} \mathrm{BME)}$ and incubated for 2 hours at room temperature. Then, the bacterial lysate was cleared by centrifugation at $15000 \mathrm{~g}$ (SS 34 rotor, Sorvall@) for 30 minutes at $4^{\circ} \mathrm{C}$ and the resultant supernatant was allowed to bind to nickel NTA-agarose for 2 hours at room temperature (or $4^{\circ} \mathrm{C}$ ). Note, under denaturing conditions the His tag on a protein is completely exposed; hence, binding to the matrix is improved. After several repeating washing steps with the urea buffer, His-tagged fusion proteins were eluted with urea buffer containing 100-250 mM imidazole. Finally, recombinant His-tagged fusion proteins purified under denaturing conditions had to be renatured and refolded. Protein renaturation and refolding was carried out via gradual dialysis against buffers with reduced urea concentrations (4-0 M urea, 1000-400 mM NaCl, $50 \mathrm{mM}$ Tris-HCl pH 7.4, $2 \mathrm{mM} \mathrm{BME)} \mathrm{at} 4^{\circ} \mathrm{C}$. Dialysis buffers were changed every 3 hours. 


\subsection{Expression and purification of nuclear transport cargoes}

\subsection{NF-Y subunits}

The NF-Y proteins were expressed in BL21 (DE3) strains (Novagen) as follows: cultures were grown at $37^{\circ} \mathrm{C}$ to an optical density at $600 \mathrm{~nm}$ of $\sim 1.0$ and then were shifted to $25^{\circ} \mathrm{C}$. After the temperature equilibrated, the cultures were induced with isopropylthio- $\beta$-D-galactoside (IPTG, $0.2 \mathrm{mM}$ for single NF-YA, NF-YB, and NF-YC cultures, $0.4 \mathrm{mM}$ for the NF-YB/NF-YC coexpression culture) and were grown for 3 hours, shaking at $230 \mathrm{rpm}$. Cells were then collected by low speed centrifugation, resuspended in buffer A $(50 \mathrm{mM}$ Tris- $\mathrm{HCl}, \mathrm{pH} 8.0,400 \mathrm{mM} \mathrm{NaCl}$, and $5 \mathrm{mM} \beta$-mercaptoethanol), and lysed by sonication $(12 \times 10$ seconds with 10 seconds break after each round). The recombinant proteins were purified on nickel NTA-agarose (Qiagen), and glutathione-Sepharose 4B (Amersham Biosciences) or GST-Bind resin (Novagen), respectively, according to the manufacturer's instructions.

To pre-assemble the trimeric NF-Y complex, recombinant NF-YA protein was immobilized on nickel NTA-agarose (Qiagen) and used as bait to fish out the NF-YB/NF-YC dimer from the soluble fraction recovered by high speed centrifugation of the co-expression lysate. After $3 \mathrm{~h}$ of incubation, the supernatant was removed and the resin was washed extensively with buffer A. The formed NF-Y trimer was eluted with imidazole and was applied onto a gel filtration column Hiload 16/60 Superdex 200 (Amersham Biosciences) equilibrated with buffer A. The purified complex was concentrated on Vivaspin (Viva Science) to a final concentration of $\sim 2 \mathrm{mg} / \mathrm{ml}$.

\subsection{Core histones}

The core histone fusion proteins were expressed in either E. coli JM109 (z-fusions) or BL21 (DE3) strains (GST-fusions). Overnight $50 \mathrm{ml}$ cultures of the selected clones were grown at $37^{\circ} \mathrm{C}$. The $50 \mathrm{ml}$ cultures were scaled up to $1000 \mathrm{ml}$ and allowed to grow until the $\mathrm{OD}_{600}$ reached a value of about 1 . Then, the cultures were induced with IPTG at a final concentration of 0.5 $1.0 \mathrm{mM}$ and incubated at $25-30^{\circ} \mathrm{C}$ for 3 hours. The bacterial lysates were prepared and the proteins were purified by affinity chromatography on nickel NTA-agarose (Qiagen) or GSTBind resin (Novagen), respectively, according to the manufacturer's instructions. In short, the bacterial pellets were resuspended in $20 \mathrm{ml}$ buffer A $(50 \mathrm{mM}$ Tris- $\mathrm{HCl} \mathrm{pH} 7.5,300-500 \mathrm{mM}$ $\mathrm{NaCl}, 5 \mathrm{mM} \beta$-mercaptoethanol), sonicated for $3 \times 60$ seconds (always with 10 second breaks after 10 seconds of sonication), and then the bacterial lysates were centrifuged at $15000 \mathrm{~g}$ (SS 34 rotor, Sorvall $\left.{ }^{\circledR}\right)$ for 30 minutes. The resultant supernatant was allowed to bind to $500 \mu 1$ of affinity matrix for 2 hours at $4^{\circ} \mathrm{C}$. The matrix was then washed several times with buffer $\mathrm{A}$, and loaded onto a column. The bound proteins were eluted in fractions of about $800 \mu 1$ with either 
$250 \mathrm{mM}$ imidazole (z-fusions) or $10 \mathrm{mM}$ reduced glutathione (GST-fusions) in buffer A. Purification under denatured conditions was done essentially as described above (chapter 2.2.3.3.4).

\subsection{Ribosomal protein L23a}

Ribosomal protein L23a was expressed in E.coli JM109 with a C-terminal His tag, and with four $\mathrm{N}$-terminal $\mathrm{z}$ tags. Expression and purification of rpL23a was similar to the expression and purification of the core histone z-fusion proteins, except for the fact that disruption of cells and purification on nickel-NTA agarose was in the presence of $1 \mathrm{M}$ lithium chloride (Jakel and Gorlich, 1998).

\subsection{Expression and purification of import receptors and supplementary factors}

The following import factors were expressed in E. coli BL21 (DE3) or E. coli JM109 as described in the literature indicated and were purified on nickel NTA-agarose, followed by chromatography on Superdex 200 (sometimes skipped, depending on the purity and activity of the import receptor): Xenopus importin $\alpha$ (Gorlich et al., 1994), human importin $\beta$ (Kutay et al., 1997b), transportin (Izaurralde et al., 1997), Xenopus importin 7, importin 5 (Jakel and Gorlich, 1998), and importin 13 (Mingot et al., 2001). Expression and purification (including affinity and ion exchange chromatography) of the following proteins was performed as described: NTF2 (Kutay et al., 1997b; Ribbeck et al., 1998), M9-GST (Pollard et al., 1996), Ran and RanQ69L(GTP) (Ribbeck et al., 1998). GST-nucleoplasmin was expressed in E. coli BL21 (DE3) for 4 hours at $25^{\circ} \mathrm{C}$ and purified on glutathione-Sepharose 4B essentially as described in chapter 2.2.3.3.3.

\subsection{Ran and NTF2}

N-terminally His-tagged Ran (wild-type and Q69L) was expressed (1 mM IPTG, 3 hours, $30^{\circ} \mathrm{C}$ ) in E. coli JM109 from pQE32 (cloned as a SphI-HindIII fragments) and purified essentially as described by Ribbeck et al. (Ribbeck et al., 1998).

Briefly, Ran (wild-type) bacterial pellets were resuspended in $50 \mathrm{mM}$ potassium phosphate $\mathrm{pH}$ 7.0, $200 \mathrm{mM} \mathrm{NaCl}, 5 \mathrm{mM} \mathrm{MgCl}$, sonicated, centrifuged, bound on nickel-NTA agarose, and eluted with $50 \mathrm{mM}$ potassium phosphate $\mathrm{pH} 7.0,5 \mathrm{mM} \mathrm{MgCl}_{2}, 250 \mathrm{mM}$ imidazole. The fractions containing Ran were pooled, diluted 5 -fold in $5 \mathrm{mM}$ potassium phosphate $\mathrm{pH} 7.0,0.5 \mathrm{mM}$ $\mathrm{MgCl}_{2}, 5 \%$ glycerol, and then applied to a $1 \mathrm{ml}$ Mono S column (Amersham Biosciences). Ran was eluted from Mono S with a linear gradient from A (20 mM potassium phosphate $\mathrm{pH} 7.0$, 
$0.5 \mathrm{mM} \mathrm{MgCl}_{2}, 5 \%$ glycerol $)$ to $\mathrm{B}\left(0.5 \mathrm{M}\right.$ potassium phosphate $\left.\mathrm{pH} 7.0,0.5 \mathrm{mM} \mathrm{MgCl}_{2}\right)$. RanGDP elutes earlier in this procedure than RanGTP.

In contrast, RanQ69L(GTP) bacterial pellets were resuspended in $50 \mathrm{mM}$ HEPES-KOH pH 7.0, $100 \mathrm{mM} \mathrm{NaCl}, 5 \mathrm{mM} \mathrm{MgCl}$, $5 \mathrm{mM} \beta$-mercaptoethanol (BME), sonicated, centrifuged, and bound on nickel-NTA-agarose. The pre-bound nucleotide was removed by washing for 1 hour with $50 \mathrm{mM}$ HEPES-KOH pH 7.0, $200 \mathrm{mM} \mathrm{NaCl}, 5 \mathrm{mM}$ BME (alternatively, EDTA could be added as well), and RanQ69L was then reloaded with GTP in the presence of $100 \mu \mathrm{M}$ GTP in $50 \mathrm{mM}$ HEPES-KOH pH 7.0, $200 \mathrm{mM} \mathrm{NaCl}, 5 \mathrm{mM} \mathrm{MgCl}_{2}, 5 \mathrm{mM} \mathrm{BME}$ (incubation again for 1 hour). After elution with $50 \mathrm{mM}$ HEPES-KOH pH 7.0, $200 \mathrm{mM} \mathrm{NaCl}, 5 \mathrm{mM} \mathrm{MgCl} 2,5 \mathrm{mM}$ BME, $250 \mathrm{mM}$ imidazole the RanQ69L(GTP) fractions were pooled, supplemented with $250 \mathrm{mM}$ sucrose and frozen in liquid nitrogen.

NTF2 was expressed and purified essentially as described by Ribbeck et al. (Ribbeck et al., 1998). In short, NTF2 was expressed in E. coli BL21 (DE3) pLysS (Novagen) (1 mM IPTG, 2 hours, $30^{\circ} \mathrm{C}$ ) untagged from a pET expression vector (Kent et al., 1996). NTF2 was precipitated from the bacterial lysate with ammonium sulfate (50\% saturation), the pellet was dissolved in $250 \mathrm{ml}$ buffer A $(50 \mathrm{mM}$ Tris $\mathrm{pH} 8.0,2 \mathrm{mM}$ DTT), NTF2 was then bound to Q Sepharose FF (HiLoad Q Sepharose Fast Flow column, Amersham Biosciences) equilibrated in buffer $\mathrm{A}$ and eluted in the $\mathrm{NaCl}$ gradient (buffer $\mathrm{B}$ : $50 \mathrm{mM}$ Tris $\mathrm{pH}$ 8.0, $500 \mathrm{mM} \mathrm{NaCl}, 2 \mathrm{mM}$ DTT) at about $230 \mathrm{mM} \mathrm{NaCl}$. Final purification was on Superdex 75 (Amersham Biosciences) where it eluted at a position expected for the homodimer. The purification of NTF2 had been done by Marc Bäuerle (previous PhD student in the Department of Molecular Biology), and this material was taken for the present work.

\subsection{Import factors}

\section{General notes}

Since expressed import factors are toxic to the cell, 'leaky' expression before induction may result in poor culture growth or in the selection of deletion mutants which grow faster than bacteria containing the correct plasmid. Thus, plasmids encoding import factors (usually pQE plasmids) were maintained in E. coli strains that either carried the pREP4 repressor plasmid or harbored the lacI ${ }^{\mathrm{q}}$ gene on the F-factor episome. To maximize repression $2 \%$ glucose was added in the recovery medium, in the transformation plates, and in the medium for overnight (pre) cultures. Further, the recombinant import receptors are only stable and active in Tris buffer (usually containing $50 \mathrm{mM}$ Tris- $\mathrm{HCl} \mathrm{pH}$ 7.5, $200 \mathrm{mM} \mathrm{NaCl}, 5 \mathrm{mM}$ magnesium chloride, and 
$5 \mathrm{mM} \mathrm{BME}$ ), and before freezing $250 \mathrm{mM}$ sucrose was generally added to the protein preparations.

\section{The importins in detail}

N-terminally His-tagged importin 13 was expressed in E. coli JM109 from pQE80 (cloned as a BamHI-HindIII fragment). For expression a pre-inoculum (starting from a single colony) was grown overnight in $50 \mathrm{ml} 2 \mathrm{YT}$ medium (or LB medium) containing $2 \%$ glucose and $100 \mu \mathrm{g} / \mathrm{ml}$ ampicillin. The $50 \mathrm{ml}$ culture was scaled up to $400 \mathrm{ml}$ of $2 \mathrm{YT}$ containing $2 \%$ glycerol, $30 \mathrm{mM}$ $\mathrm{K}_{2} \mathrm{HPO}_{4}$, and $100 \mu \mathrm{g} / \mathrm{ml}$ ampicillin and was allowed to grow at $37^{\circ} \mathrm{C}$ until the $\mathrm{OD}_{600}=1$. Then, $600 \mathrm{ml}$ of the same medium was added and the culture was transferred to $16^{\circ} \mathrm{C}$. After 2 hours growing under these conditions expression of the protein was induced with IPTG at a final concentration of $0.1 \mathrm{mM}$, and the culture was further incubated at $16^{\circ} \mathrm{C}$ for $12-14$ hours. The bacterial lysate was prepared (disruption of the cells by ultrasonic sound) and the protein was purified in two steps. First, by affinity chromatography on nickel NTA-agarose (equilibration buffer contained $50 \mathrm{mM}$ Tris- $\mathrm{HCl} \mathrm{pH} 7.5,500 \mathrm{mM} \mathrm{NaCl}, 10 \mathrm{mM}$ magnesium acetate, $5 \mathrm{mM}$ BME, and $5 \%$ glycerol), followed by chromatography (gel filtration) on Superdex 200 equilibrated with $50 \mathrm{mM}$ Tris- $\mathrm{HCl} \mathrm{pH}$ 7.5, $100 \mathrm{mM} \mathrm{NaCl}$, and $3 \mathrm{mM}$ magnesium acetate.

C-terminally His-tagged importin $\boldsymbol{\beta}$ and N-terminally His-tagged transportin were expressed in E. coli M15[pREP4] from pQE60 and from pQE32, respectively. For expression a pre-inoculum (starting from a single colony) was grown overnight in $200 \mathrm{ml}$ 2YT medium (or LB medium) containing $2 \%$ glucose, $100 \mu \mathrm{g} / \mathrm{ml}$ ampicillin, and $50 \mu \mathrm{g} / \mathrm{ml}$ kanamycin. Next morning the preinoculum $\left(\mathrm{OD}_{600} \sim 1.2\right)$ was used to inoculate $600 \mathrm{ml}$ of pre-warmed 2YT medium containing the appropriate antibiotics, and the bacteria were grown at $37^{\circ} \mathrm{C}$ until $\mathrm{OD}_{600}=1$ (1-2 hours). Then $800 \mathrm{ml}$ of ice-cold LB medium containing $4 \%$ ethanol and antibiotics was added, and the culture was shifted to $21^{\circ} \mathrm{C}$. After the temperature had equilibrated $0.5 \mathrm{mM}$ IPTG (maximum concentration) was added and the protein was expressed for 4 hours. Cells were then collected by low speed centrifugation, resuspended in sonication buffer $(50 \mathrm{mM}$ Tris- $\mathrm{HCl}, \mathrm{pH} 7.5,500 \mathrm{mM}$ $\mathrm{NaCl}, 5 \%$ glycerol, and $5 \mathrm{mM}$-mercaptoethanol), and freeze-thawed using liquid nitrogen. The bacterial lysate was prepared by sonication and the protein was purified in two steps. First, by affinity chromatography on nickel NTA-agarose (equilibrated in sonication buffer), followed by chromatography (gel filtration) on Superdex 200 equilibrated with $50 \mathrm{mM}$ Tris- $\mathrm{HCl} \mathrm{pH} 7.5$, $200 \mathrm{mM} \mathrm{NaCl}, 5 \mathrm{mM}$ magnesium chloride, and $5 \mathrm{mM}$ BME. 
C-terminally His-tagged importin $\boldsymbol{\alpha}$ was expressed in E. coli M15[pREP4] from pQE70 (cloned as a SphI-BamHI fragment). For expression a pre-inoculum (starting from a single colony) was grown overnight at $37^{\circ} \mathrm{C}$ in $50 \mathrm{ml} 2 \mathrm{YT}$ medium containing $100 \mu \mathrm{g} / \mathrm{ml}$ ampicillin and $50 \mu \mathrm{g} / \mathrm{ml}$ kanamycin. The $50 \mathrm{ml}$ culture was scaled up to $400 \mathrm{ml}$ of $2 \mathrm{YT}$ containing the appropriate antibiotics and the bacteria were grown at $26^{\circ} \mathrm{C}$ to an optical density of 0.9 (at $600 \mathrm{~nm}$ ). The expression of the protein was induced with $1 \mathrm{mM}$ IPTG at the same temperature for 3 hours. The bacterial cells were pelleted and resuspended in buffer A $(50 \mathrm{mM}$ Tris- $\mathrm{HCl}, \mathrm{pH} 7.5,500 \mathrm{mM}$ $\mathrm{NaCl}, 5 \%$ glycerol, and $5 \mathrm{mM} \beta$-mercaptoethanol). The suspension was freeze-thawed and the bacterial lysate was prepared by sonication (disruption of the cells by ultrasonic sound for $2 \times 60$ seconds). The bacterial lysate was clarified by centrifugation at $15000 \mathrm{~g}$ for 30 minutes. The resultant supernatant was allowed to bind to $500 \mu$ l of nickel-NTA agarose (equilibrated in buffer A) for 2 hours at $4^{\circ} \mathrm{C}$. The affinity matrix was then washed several times with buffer A, and loaded onto a column. Importin $\alpha$ was eluted in fractions of about $500 \mu 1$ with buffer B (50 mM Tris- $\mathrm{mCl}, \mathrm{pH} 7.5,200 \mathrm{mM} \mathrm{NaCl}, 5 \mathrm{mM}$ magnesium chloride, and $5 \mathrm{mM} 3-$ mercaptoethanol) containing $250 \mathrm{mM}$ imidazole. Finally, pooled fractions of importin $\alpha$ were dialyzed against several changes of buffer B, and as usually before freezing $250 \mathrm{mM}$ sucrose was added.

Purified importin $\alpha$ (see protocol above) was a kind gift from Sonja Neimanis (PhD student in the Department of Molecular Biology).

Expression and purification of N-terminally His tagged importin 7 and importin 5 from pQE9 respectively $\mathrm{pQE} 32$ was identical to importin $\beta$, except for the fact that expression of these import factors was induced at $17^{\circ} \mathrm{C}$ and not allowed to proceed for more than 3 hours.

\subsubsection{Binding assays}

\subsubsection{GST pulldowns}

Bacteria expressing the recombinant GST fusion proteins were lysed in buffer A $(50 \mathrm{mM}$ Tris$\mathrm{HCl}, \mathrm{pH} 8.0,400 \mathrm{mM} \mathrm{NaCl}, 5 \mathrm{mM}$ ß-mercaptoethanol), centrifuged and then the supernatant was incubated with glutathione-Sepharose 4B beads or GST-Bind resin, respectively. Bound proteins $(\sim 0,5 \mathrm{mg} / \mathrm{ml}$ of resin) were washed three times in binding buffer B (50 mM Tris- $\mathrm{HCl}$, $\mathrm{pH}$ 7.5, $300 \mathrm{mM} \mathrm{NaCl}, 5 \mathrm{mM} \mathrm{MgCl}, 5 \mathrm{mM}$ ß-mercaptoethanol) and used as affinity matrix for the binding experiments. For each binding experiment appropriate amounts of affinity matrix $(0,2 \mu \mathrm{M}$ final concentration of bound protein) were incubated with either pre-cleared bacterial lysates containing expressed import receptors $(\sim 200 \mu \mathrm{l})$ or the corresponding purified 
recombinant import factor in buffer $\mathrm{B}\left(0,2 \mu \mathrm{M}\right.$ final concentration) at $4^{\circ} \mathrm{C}$ for 3 hours in the presence or absence of $2 \mu \mathrm{M}$ RanQ69L(GTP). Supernatants were removed and the beads were washed four times with ice-cold buffer B. Proteins were eluted with SDS-PAGE sample buffer, boiled for $10 \mathrm{~min}$, and analyzed by SDS-PAGE.

\subsubsection{Binding studies on IgG Sepharose}

Bacteria expressing the recombinant $2 \mathrm{z} / 6 \mathrm{z}$-fusion proteins were lysed in binding buffer $(50 \mathrm{mM}$ Tris-Cl, $\mathrm{pH} 7.5,300 \mathrm{mM} \mathrm{NaCl}, 5 \mathrm{mM} \mathrm{MgCl} 2,5 \mathrm{mM} \beta$-mercaptoethanol), and the cleared bacterial lysate was incubated with IgG-Sepharose beads to a final concentration of $0.2-1.0 \mu \mathrm{g} / \mu \mathrm{l}$ of resin (matrix). The pre-loaded matrix was washed twice with binding buffer and was used as affinity matrix for the binding experiments. For each binding experiment about $20 \mu \mathrm{l}$ of the respective affinity matrix was rotated with either $200-600 \mu l$ of pre-cleared bacterial lysate containing expressed import receptors or $150 \mathrm{pmol}$ of the corresponding purified recombinant import factor in binding buffer for $3 \mathrm{~h}$ at $4^{\circ} \mathrm{C}$ in the presence or absence of $1.5 \mu \mathrm{M}$ RanQ69L(GTP). The beads were recovered by gentle centrifugation, and then washed four times with $1 \mathrm{ml}$ of binding buffer, resuspended in $20 \mu \mathrm{l}$ of SDS-PAGE sample buffer, boiled for 5 minutes, centrifuged, and the resultant supernatant was then analyzed by SDS-PAGE. Alternatively, the bound material was eluted with $1.5 \mathrm{M} \mathrm{MgCl}_{2}$ in $50 \mathrm{mM}$ Tris- $\mathrm{HCl} \mathrm{pH} 7.5$. Proteins were precipitated with $90 \%$ isopropanol (final concentration), dissolved in SDS-PAGE sample buffer and analyzed by SDS-PAGE.

\subsubsection{Preparation of fluorescence-labeled import substrates}

Cargo substrates that did not contain a GST tag were fluorescence-labeled in principle as previously described (Bauerle et al., 2002). Briefly, purified 6z-fusion proteins expressed in E. coli, except for the cores histones, were dialyzed against $20 \mathrm{mM} \mathrm{HEPES-KOH,} \mathrm{pH} \mathrm{7.4,}$ $110 \mathrm{mM}$ potassium acetate, $5 \mathrm{mM}$ magnesium acetate and incubated overnight at $4^{\circ} \mathrm{C}$ with a 10-fold molar excess of fluorescein isothiocyanate (Sigma) or alternatively with a 10-fold molar excess of FLUOS (carboxy fluorescein $N$-hydroxysuccinimide ester, dissolved in DMSO). Both of these acylating reagents are amine-reactive probes and form thioureas or rather carboxamides upon reaction with amines. However, amide bonds in carboxamides are as stable as peptide bonds; hence, succinimidyl esters (such as FLUOS) have become the preferred reagents for modification of amines. Core histone 6z-fusion proteins were dialyzed against $50 \mathrm{mM}$ HEPES-KOH pH 7.5, $500 \mathrm{mM} \mathrm{NaCl}$ and were also incubated over night at $4^{\circ} \mathrm{C}$ with a 10 -fold molar excess of fluorescein isothiocyanate. Labeling of 4z-rpL23a and linker histone H1 with 
fluorescein-5'-maleimide (Pierce) through their engineered cysteine residues was performed in $50 \mathrm{mM}$ HEPES-KOH, pH 7.5, $300 \mathrm{mM}$ potassium acetate, $5 \mathrm{mM}$ magnesium acetate. The advantage of labeling through sulfhydryl groups of the engineered cysteine residues is that both proteins are specifically labeled in contrast to the amine-reactive probes.

The labeled reaction mixtures were passed over NAPTM 5 columns (Amersham Biosciences) equilibrated in $20 \mathrm{mM}$ HEPES, $\mathrm{pH} 7.3,110 \mathrm{mM}$ potassium acetate, $2 \mathrm{mM}$ magnesium acetate, $1 \mathrm{mM}$ EGTA, $2 \mathrm{mM}$ dithiothreitol, and $250 \mathrm{mM}$ sucrose (respectively equilibrated in the buffer used to dialyze the cargoes against) to remove unincorporated fluorochrome.

\subsubsection{Import assays}

Import reactions were performed at $37^{\circ} \mathrm{C}$ essentially as described previously (Jakel and Gorlich, 1998) based on the method established by Adam et al. (Adam et al., 1990). This assay allows reconstitution of nuclear import events by using recombinant purified transport factors without the interference of endogenous importins.

Briefly, HeLa cells were grown on $10 \mathrm{~mm}$ glass coverslips to $40-80 \%$ confluence. Permeabilization of the plasma membrane was done with $40 \mu \mathrm{g} / \mathrm{ml}$ digitonin (Calbiochem) for 10 min on ice. The treatment with the non-ionic detergent digitonin selectively permeabilizes the cholesterol-rich plasma membranes but leaves intact the nuclear membrane. Permeabilization allows soluble cytoplasmic proteins to leak out of the cell and to be washed away. The cells were kept on ice for efficient cytosol depletion and were washed three times with ice-cold transport buffer (20 mM HEPES-KOH, pH 7.4, $110 \mathrm{mM}$ potassium acetate, $5 \mathrm{mM}$ magnesium acetate, 0,5 mM EGTA, $2 \mathrm{mM}$ dithiothreitol, and $250 \mathrm{mM}$ sucrose). The amount of remaining soluble proteins in the cytoplasm depends on the digitonin concentration and incubation time applied, generally ranging between $20-30$ percent. The permeabilized cells were incubated in a humidity chamber for 25 min with $20 \mu \mathrm{l}$ of a transport reaction mix consisting of cargo $(2 \mu \mathrm{M})$ and either recombinant importin $(2 \mu \mathrm{M})$ or $10 \mu \mathrm{l}$ of reticulocyte lysate (Promega) as general source of import receptors in transport buffer along with an energy-regenerating system (0.5 mM ATP, $0.5 \mathrm{mM}$ GTP, $10 \mathrm{mM}$ creatine phosphate, and $50 \mu \mathrm{g} / \mathrm{ml}$ creatine kinase). In case of reconstitution experiments with recombinant transport factors $3 \mu \mathrm{M} \operatorname{Ran}(\mathrm{GDP})$ and $0.5 \mu \mathrm{M}$ NTF2 (called Ran mix) were added to the transport reaction mix to create a RanGTP gradient across the nuclear membrane. Recombinant purified proteins gain intracellular access when overlaid on the digitonin-permeabilized cells. Cells were fixed with $3 \%$ paraformaldehyde for 15 min and were mounted by using Vectashield with DAPI (Vector Laboratories). For the negative controls the assay was done in the absence of any transport factors, i.e. without 
reticulocyte lysate and without recombinant transport factors. Import reactions were visualized by either direct (fluorescently labeled cargoes) or indirect (non-fluorescent transport substrates, detected with antibodies) fluorescence microscopy with a Zeiss microscope (Axioskop 20) using a $40 \times$ objective lens (Plan Neofluar) as extensively described in chapter 2.2.7.

\section{Inhibition experiments}

The in vitro nuclear import inhibition experiments were done as follows. For energy depletion, the energy-regenerating system was omitted from the transport mix. Instead, the permeabilized cells as well as the reticulocyte lysate were pre-treated with $100 \mathrm{U} / \mathrm{ml}$ apyrase (Sigma) for 15 minutes at room temperature. Blocking of the nuclear transport was achieved by treatment (pre-incubation) of the permeabilized cells for $15 \mathrm{~min}$ at room temperature with $100 \mu \mathrm{g} / \mathrm{ml}$ wheat germ agglutinin (WGA). This lectin binds specifically to glycosylated residues of several nucleoporins, thereby inhibiting the translocation of cargo molecules through the nuclear pore complexes (Finlay et al., 1987). It is not known whether the blocked nuclear transport is caused by competition of WGA with import receptors or by steric interference. WGA treatment prevents receptor-mediated active nuclear transport but still allows passive diffusion (Palacios et al., 1997). Further inhibition experiments included the incubation of the import mixture at $4^{\circ} \mathrm{C}$ and addition of RanQ69L-GTP ( $15 \mu \mathrm{M}$ final concentration), a Ran mutant that is not able to hydrolyze GTP and therefore is arrested in the GTP-bound state (Bischoff et al., 2002). For competition experiments 10-times the concentration of the actual cargo of non-fluorescent competitor protein was added to the import reaction ( $\sim 5 \mu \mathrm{M}$ final concentration).

\subsubsection{Immunodetection/Immunofluorescence}

The subcellular localization of the gene products, endogenous NF-Y subunits, and non-fluorescent transport substrates was detected by indirect immunofluorescence either after 24 hours or 48 hours of incubation at $37^{\circ} \mathrm{C}$. For that, the cells were briefly washed in phosphatebuffered saline (PBS, $140 \mathrm{mM} \mathrm{NaCl}, 2.7 \mathrm{mM} \mathrm{KCl}, 1.5 \mathrm{mM} \mathrm{KH}_{2} \mathrm{PO}_{4}$, and $9 \mathrm{mM} \mathrm{Na}_{2} \mathrm{HPO}_{4} \mathrm{pH}$ 7.4) and fixed with $3 \%$ paraformaldehyde in PBS for 15 minutes at room temperature. Before and after permeabilization with $0.5 \%(\mathrm{v} / \mathrm{v})$ Triton X-100 in PBS for 10 minutes, cells were washed three times with PBS. Afterwards unspecific binding was blocked with blocking buffer [3\% bovine serum albumin (BSA) in PBS] for 15 minutes at room temperature. The cells were then incubated with an appropriate dilution of primary antibody in blocking buffer for one hour at $37^{\circ} \mathrm{C}$. For visualization cells were washed with PBS and incubated for another hour at $37^{\circ} \mathrm{C}$ with a fluorescently labeled secondary antibody diluted in blocking buffer. Monoclonal mouse 
anti-NF-YA (BD Pharmingen), polyclonal rabbit anti-CBF-A (Santa Cruz), polyclonal rabbit anti-CBF-C (Santa Cruz), monoclonal mouse anti- $\beta$-galactosidase (Sigma), monoclonal mouse anti-HA (Santa Cruz), monoclonal mouse anti-Myc (Invitrogen), and monoclonal mouse antiGST (Santa Cruz) antibodies were used as primary antibodies. Cy3-labeled goat anti-mouse (Dianova), AlexaFluor488-labeled goat anti-mouse (Molecular Probes), AlexaFluor555-labeled goat anti-mouse (Molecular Probes), AlexaFluor488-labeled goat anti-rabbit (Molecular Probes), and AlexaFluor555-labeled goat anti-rabbit (Molecular Probes) antibodies were used as secondary antibodies. The nuclei were visualized with the DNA-binding substrate diamino-2phenylindol (DAPI, Sigma) that was part of the embedding medium (90\% glycerol, $10 \%$ PBS, $1 \mathrm{mg} / \mathrm{ml}$ phenylenediamin, $1 \mu \mathrm{g} / \mathrm{ml}$ DAPI). Immunofluorescence was evaluated with a Zeiss microscope (Axioskop 20) using a 40 x objective lens (Plan Neofluar). Pictures were obtained by digital imaging (the program SPOT was used as software).

\subsubsection{In vitro transcription and translation}

In vitro transcription-translation of the different NF-YA, NF-YB, NF-YC constructs (pJK19, pJK20, pJK143, pJK154, pJK155, pJK163, and pJK164), TFIIIA, and GST was performed from the corresponding SP6 promoter constructs $(\mathrm{pCS} 2+\mathrm{MT})$. Using the $\mathrm{T}_{\mathrm{N}} \mathrm{T}$ coupled reticulocyte lysate system (Promega) according to the manufacturer's instructions the proteins were labeled with $2 \mu \mathrm{l}\left[{ }^{35} \mathrm{~S}\right]$ methionine $(10 \mu \mathrm{Ci} / \mathrm{ml})$ (Amersham Biosciences). Reactions were performed at $30^{\circ} \mathrm{C}$ for 2 hours in a $25 \mu 1$ volume and the samples were then either frozen or directly used for oocyte microinjections. To control the efficiency of in vitro transcription and translation $1 \mu 1$ of each translation mix was analyzed by SDS-PAGE and subsequent autoradiography/ phosphorimaging (see also chapter 2.2.3.1).

\subsubsection{Oocyte microinjections and analysis}

Preparation of oocytes, microinjection and immunoprecipitation were performed as described before (Gurdon, 1977; Wischnewski et al., 2004).

\subsubsection{Preparation and collagenase treatment of Xenopus oocytes}

The preparation of the oocytes was done by Susanne Loop and Katja Horvay (Department of Developmental Biochemistry), essentially as described by Terns and Goldfarb (Terns and Goldfarb, 1998).

The isolation of the single oocytes for the microinjection experiments can be achieved by collagenase treatment, which eventually results in the separation of individual oocytes from the 
ovarian tissue. Treatment was with $1 \mathrm{mg} / \mathrm{ml}$ collagenase (Sigma) in $5 \mathrm{mM}$ HEPES pH 7.5, $82.5 \mathrm{mM} \mathrm{NaCl}, 2.5 \mathrm{mM} \mathrm{KCl}$, and $1 \mathrm{mM} \mathrm{MgCl}_{2}$ for 2 hours at room temperature (rotating). To completely remove the collagenase after treatment, oocytes were washed three times with MBSH buffer (10 mM HEPES pH 7.6, 88 mM NaCl, 2.4 mM NaHCO $3,1 \mathrm{mM} \mathrm{KCl,} 0.8 \mathrm{mM} \mathrm{MgSO}_{4}$, $0.4 \mathrm{mM} \mathrm{CaCl}_{2}$, and $0.6 \mathrm{mM} \mathrm{KNO}_{3}$ ). For recovery, occytes were kept overnight at $18^{\circ} \mathrm{C}$ in MBSH, a buffer in which they can be stored for several days.

\subsubsection{Microinjection in Xenopus oocytes}

Only healthy stage V and stage VI oocytes (for definition of the different stages, see Dumont, 1972) were selected for injection. Healthy oocytes are homogeneously pigmented on their animal (dark) hemisphere, whereas dying oocytes exhibit mottled pigmentation. For injection, the closed tip of the glass needle (previously prepared with the needle puller) was broken, loaded, and samples of 10-20 $\mathrm{nl}$ of the in vitro translation reaction (see chapter 2.2.7) were injected into either cytoplasm (vegetal hemisphere; white) or nucleus (animal hemisphere; dark) of oocytes. Immediately after injection and at the indicated time points, nucleus and cytoplasm of 10-15 oocytes were dissected manually and homogenized (by pipetting up and down) in NET-2 buffer (50 mM Tris-HCl, pH 7.4, 150 mM NaCl, $0.05 \%$ NP-40).

\subsubsection{Immunoprecipitation}

Proteins of the nuclear and cytoplasmic fractions of the injected oocytes were precipitated using antibodies against the myc-tag (Santa Cruz) and finally analyzed by SDS-PAGE and autoradiography/phosphorimaging (Amersham Biosciences). Briefly, after homogenization in NET-2 buffer the probes were centrifuged for 5 minutes at $14000 \mathrm{rpm}$ and the cleared supernatant was transferred to a GammaBind ${ }^{\mathrm{TM}}$ Plus Sepharose ${ }^{\mathrm{TM}}$ (Amersham Biosciences) pellet pre-loaded with monoclonal mouse anti-Myc (Invitrogen) antibodies. The tubes were sealed with parafilm and incubated at $4^{\circ} \mathrm{C}$ for 1 hour on a rotating wheel. Then, the pellet was washed three times with ice-cold NET-2 buffer, the buffer was removed, the myc-tagged fusion proteins were eluted with SDS-PAGE sample buffer, boiled for 5 minutes, and finally analyzed by SDS-PAGE. Prior to phosphorimaging, the gel was first dried for 2 hours on the gel dryer and then exposed on a phosphorscreen for up to 3 days. 


\section{Results}

\subsection{Nuclear transport of the transcription factor NF-Y}

Transcription factors are among the classes of proteins whose role is clearly nuclear. Regarding the nuclear transport of transcription factors an oversimplified picture would look as follows. Nuclear import of a transcription factor triggers transcriptional response while nuclear export (restriction to the cytoplasm) would prevent transcriptional response. In case of the CCAATspecific transcription factor NF-Y the functional complex consists of three subunits NF-YA, NF-YB, and NF-YC. Only the heterotrimeric complex has a high affinity and sequence specificity for the CCAAT boxes (Bi et al., 1997; Dorn et al., 1987; Kim and Sheffery, 1990), hence, the presence of all three subunits in the nucleus is a prerequisite for transcriptional activation by NF-Y. To investigate the nuclear transport of the heterotrimeric transcription factor it was therefore reasonable and fundamental to characterize the nuclear import of the single subunits first, and the initial question concerned the subcellular localization of NF-YA, NF-YB, and NF-YC.

\subsubsection{In vivo studies}

\subsubsection{NF-Y subunits are predominantly nuclear at steady state}

The subcellular localization of endogenous NF-Y subunits and differently tagged, overexpressed versions of the three subunits was examined in HeLa cells. While the subcellular localization of endogenous NF-Y subunits was detected with appropriate antibodies, HeLa cells were also transiently transfected (chapter 2.2.1.2.4) with plasmid DNA encoding the three differently tagged subunits. Besides $\beta$-galactosidase (ß-gal), the genes encoding enhanced green fluorescent protein (EGFP), hemagglutinin (HA), and the so-called myc-tag were used as reporter genes (chapter 2.2.2.15) and the subcellular localization of the gene products (tagged fusion proteins) was examined 24 hours after transient transfection by either direct (EGFP) or indirect ( $ß$-gal, HA, myc) fluorescence microscopy (chapter 2.2.7). The $\beta$-galactosidase-tagged fusion constructs were under control of a SV40 promoter that caused a moderate over-expression of the ß-gal-fusion proteins. In contrast, EGFP-, HA-, and myc-tagged fusion proteins were strongly over-expressed in HeLa cells because their genes were under control of a strong CMV promoter. Regarding the subcellular localization, dominant nuclear localization patterns were evident for the endogenous and $\beta$-galactosidase-tagged NF-YA, NF-YB and NF-YC subunits (Fig.4A). In contrast, strong over-expression of the NF-Y subunits influenced the distribution of NF-YB and significantly of NF-YC, while the dominant nuclear localization pattern of NF-YA remained unchanged (Fig.4B). 


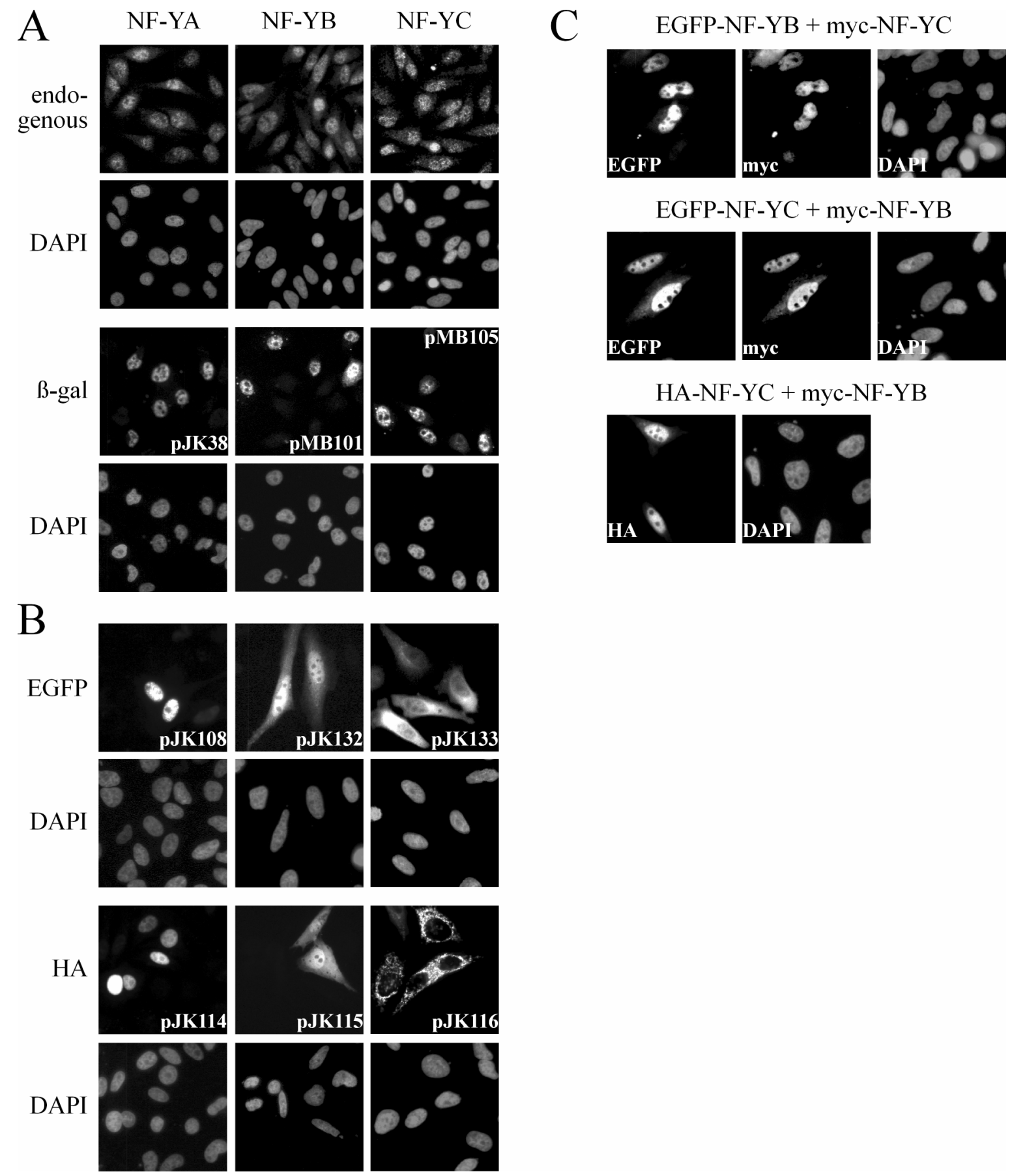

FIG. 4. Subcellular localization of NF-Y subunits in HeLa cells. (A) The endogenous NF-Y subunits stained with anti-NF-YA, anti NF-YB, and anti NF-YC antibodies and moderately over-expressed (SV40 promotor) B-galactosidase-tagged NF-Y subunits are predominantly nuclear at steady state in HeLa cells. (B) The subcellular localization of strongly over-expressed (CMV promotor) EGFP-tagged and HA-tagged NF-Y subunits differs in HeLa cells. (C) EGFP-NF-YB, EGFP-NF-YC and HA-NF-YC were co-transfected with the corresponding myctagged histone fold partner and co-stained as indicated. In all the cases the subcellular localization of the tagged fusion proteins was examined 24 hours after transient transfection, either by direct (EGFP) or indirect (ß-gal, HA, myc) fluorescence microscopy. Nuclei were stained with DAPI. 
The fluorescent staining of EGFP-tagged NF-YB (EGFP-NF-YB) and HA-tagged NF-YB (HA-NF-YB) became more cytoplasmic compared to the ß-galactosidase fusion protein and EGFP-tagged NF-YC (EGFP-NF-YC) and HA-tagged NF-YC (HA-NF-YC) remained essentially in the cytoplasm (Fig.4B).

However, when EGFP-NF-YB was over-expressed together with myc-tagged NF-YC (Fig.4C, upper panels) and EGFP-NF-YC was co-expressed with myc-tagged NF-YB (Fig.4C, middle panels), the subcellular localization changed, becoming largely nuclear again (Fig.4C; note the co-localization of the co-transfected myc-tagged subunits). This behavior could also be observed co-expressing HA-NF-YC and myc-tagged NF-YB (Fig.4C lowest panel, co-staining was not possible because of the same secondary antibody) which further indicates that the nuclear localization of NF-YC depends on the presence of its histone fold partner NF-YB, as previously shown by Frontini et al. (Frontini et al., 2004). Taken together, these results point toward NF-YA being imported into the nucleus independently of the two HFM containing subunits, whereas NF-YB and NF-YC mediate the nuclear localization of its corresponding histone fold partner. In particular the nuclear localization of NF-YC seems to be completely restricted to the presence of NF-YB. Co-expression of NF-YB shifted the subcellular localization of strongly over-expressed NF-YC fusion proteins again to predominantly nuclear at steady state as observed for the endogenous and the moderately over-expressed monomeric NF-YC subunit.

\subsubsection{Explanations for the cytoplasmic localization of over-expressed NF-YC}

What is the reason for the cytoplasmic localization of strongly over-expressed NF-YC? In principle, two explanations could be suggested: Firstly, NF-YC is devoid of a nuclear localization signal (NLS) and needs the histone fold partner NF-YB to be imported into the nucleus. Secondly, NF-YC can enter (passively or receptor mediated) the nucleus, but active nuclear export due to a nuclear export signal (NES) re-localizes the subunit to the cytoplasm, when in excess to the heterodimerization capacity of NF-YB as suggested by Frontini et al. (Frontini et al., 2004). To address the question whether active nuclear export or the lack of a NLS was responsible for the cytoplasmic localization of strongly over-expressed NF-YC, two independent experiments were performed. On one hand, we examined the effects of the nuclear export inhibitor leptomycin B (LMB) on the subcellular localization of HA-NF-YC (Fig.5C), and on the other hand we studied nuclear export of NF-YC labeled with ${ }^{35}$ S-methionine by in vitro transcription/translation (see chapter 2.2.8) (along with NF-YB) by microinjections into the nuclei of Xenopus oocytes (Fig.5A). 
After microinjection, nuclear and cytoplasmic fractions of 15 injected oocytes were separated manually at the indicated time points, were homogenized by pipetting up and down, the injected myc-tagged proteins were immunoprecipitated using matrix-bound antibodies against the myctag, and were finally analyzed by SDS-PAGE with subsequent phosphorimaging (chapter 2.2.9). As shown in Fig.5A, 6 hours after microinjection the majority of the nucleoplasmically injected HFM containing subunits were still detected in the nuclear fractions. Note that the amount of

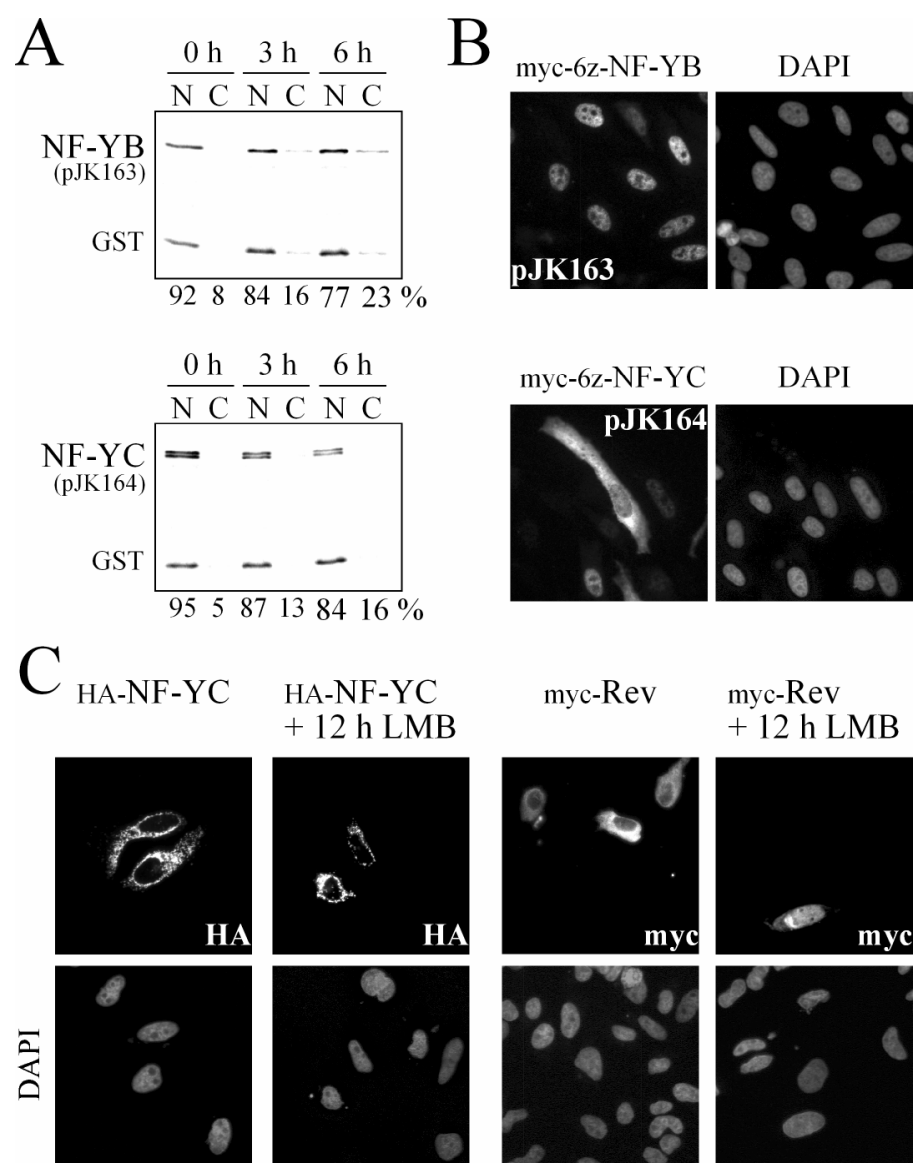

FIG. 5. Nuclear export does not cause the cytoplasmic localization of over-expressed NF-YC. (A)

${ }^{35}$ S-labeled 6z-NF-YB, 6z-NF-YC myc-tagged fusion proteins were injected into the nucleus of Xenopus oocytes. Nuclear $(\mathrm{N})$ and cytoplasmic (C) fractions were separated immediately $(0 \mathrm{~h})$ and after three $(3 \mathrm{~h})$ and six hours $(6 \mathrm{~h})$ of incubation. To control the injection/separation of nuclear and cytoplasmic fractions, GST protein was co-injected into the nucleus. The nucleocytoplasmic distribution of the injected proteins was quantified with the program ImageQuant 5.2 after phosphorimaging (in percent, written below the gels). NF-YB and NF-YC were not significantly exported from the nucleus but the majority of the injected HFM containing subunits were still detected in the nuclear fractions six hours later. (B) Subcellular localization of strongly over-expressed (CMV promotor) myc-6z-NF-YB and myc-6z-NF-YC in HeLa cells. (C) In transiently transfected HeLa cells, HA-NF-YC showed cytoplasmic staining. Inactivation of CRM1 (exportin1) by $10 \mathrm{ng} / \mathrm{ml}$ leptomycin B (LMB) did not change this localization, even after 12 hours of treatment. In contrast, LMB treatment altered the localization of the CRM1 cargo HIV-1 Rev (myc-tagged) from exclusively cytoplasmic to mainly nuclear. 
proteins found in the cytoplasmic fractions roughly corresponded to the amount of co-injected GST found in the cytoplasm. This, however, indicates that the material found in the cytoplasm was rather leaking out of the nucleus (perhaps due to some kind of nuclear membrane damage) than being actively exported. Since NF-YB and NF-YC were not significantly exported from the nucleus we can conclude that these subunits probably do not contain a nuclear export signal (NES). For these microinjections into the nuclei of oocytes the NF-YB/NF-YC fusion proteins were additionally tagged with six $z$ domains besides the six myc-tags (chapter 2.2.2.15). This was necessary because, in contrast to the cytoplasmic injections (see chapter 3.1.1.5 and Fig.9), HFM containing subunits that were injected into the nucleus without this size enlargement passively diffused out of the nuclei (data not shown). To see whether this additional 6z-tag had an influence on the overall behavior of the proteins, the constructs used for in vitro transcription/translation of the subsequently injected proteins were also transiently transfected in HeLa cells. As shown in Fig.5B, the fluorescent staining of NF-YB was nuclear whereas NF-YC was mainly found in the cytoplasm. However, myc-6z-NF-YC was also localized to the nucleus when less strongly over-expressed which is in accordance to the data previously obtained using differently tagged fusion constructs (for comparison, see again Fig.4). Hence, the addition of $6 z$ tags did not influence the nuclear import of the two HFM containing subunits which implies that also nuclear export should not be negatively influenced by the additional tag.

Besides the microinjection experiments LMB treatment was applied because several stretches of amino acid residues similar to the leucine-rich NES consensus sequence $\Phi \mathrm{X}_{2-3} \Phi \mathrm{X}_{2-3} \Phi \mathrm{X} \Phi$ $(\Phi=\mathrm{L}, \mathrm{I}, \mathrm{V}, \mathrm{F}, \mathrm{M})$ (Bogerd et al., 1996; Kim et al., 1996a; Zhang and Dayton, 1998) can be found in NF-YB and NF-YC. However, in contrast to classical NLSs the functional characterization of many putative NESs has made clear that extreme care should be taken in assigning a nuclear export function to leucine-rich sequence morifs. In other words, a sequence based prediction of a functional leucine-rich NES is impossible. CRM1/exportin-1 is the export receptor for leucine-rich NES (Fornerod et al., 1997a; Fukuda et al., 1997; Ossareh-Nazari et al., 1997). LMB directly binds (covalently) to CRM1 and thereby interferes with the formation of the trimeric RanGTP/CRM1/NES export complex (Fornerod et al., 1997b) (see also chapter 1.6.2). As shown in Fig.5C, inactivation of CRM1 by $10 \mathrm{ng} / \mathrm{ml} \mathrm{LMB}$ did not even after 12 hours of treatment change the cytoplasmic localization of HA-NF-YC which had been transiently expressed in HeLa cells. On the other hand, LMB treatment changed the localization of the known CRM1 cargo HIV-1 Rev (in which the prototype leucine-rich NES was discovered first) from exclusively cytoplasmic to mainly nuclear (Fig.5C, right panels). These results strongly indicate that the cytoplasmic localization of NF-YC was not due to CRM1-mediated nuclear 
export. Contrary to our observations, Frontini et al. reported that transiently transfected NF-YC-GFP accumulates in the nucleus of LMB treated NIH3T3 cells (Frontini et al., 2004). However, since LMB treatment was not used as sole assessment but along with microinjection experiments in Xenopus oocytes, we conclude that not active nuclear export causes the cytoplasmic localization of strongly over-expressed NF-YC, but most likely the lack of an independent NLS.

Taken together, the foregoing results suggested that NF-YC despite being devoid of a NLS could somehow mediate the nuclear localization of NF-YB while nuclear accumulation of NF-YC entirely depended on the presence of its corresponding histone fold partner NF-YB. In contrast, nuclear import of NF-YA was independent of the two HFM containing subunits and other endogenous proteins because strong over-expression of NF-YA did not result in a reduced nuclear accumulation. Thus, the next question concerned the protein regions or sequence motifs that are involved in nuclear targeting of the individual NF-Y subunits.

\subsubsection{Mapping of nuclear localization signals (NLSs)}

To identify the regions or sequence motifs that are essential for the nuclear localization of the three NF-Y subunits, we constructed a series of proteins which contained fragments of the individual NF-Y subunits fused to bacterial $\beta$-galactosidase as reporter protein and expressed the gene constructs in HeLa cells. As previously shown, dominant nuclear localization patterns were evident for the full length B-galactosidase-tagged NF-YA, NF-YB and NF-YC subunits which were moderately over-expressed in HeLa cells (see again Fig.4A). The results of these in vivo transfection experiments, summarized in Fig.6 and Fig.7, revealed that the nuclear targeting signal of each subunit corresponded to its evolutionarily conserved domain.

Precisely, the region required for nuclear localization of NF-YB and NF-YC consists of their known protein-protein interaction domain termed histone fold motif (HFM). Constructs containing the amino- or carboxy-terminus of the protein or only parts of the HFM were not imported into the nucleus (except for the partial nuclear staining observed for pMB117, Fig.6B), but remained in the cytoplasm (Fig.6A and 6B). Thus, in the two HFM containing subunits NF-YB and NF-YC the regions necessary for nuclear localization correspond to their HFMs. Since NF-YB and NF-YC dimerize tightly head to tail via these structural motifs and no additional regions involved in nuclear targeting could be identified in these two NF-Y subunits, another question arose. How can either NF-YB or NF-YC then mediate nuclear accumulation of its corresponding histone fold partner? Further experiments in this study were directed towards answering this question. 
A

NF-YB- $ß$-galactosidase-fusions

$\begin{array}{ll}\begin{array}{ll}\text { name of } \\ \text { construct }\end{array} & \text { aa } \\ \text { pMB102 } & 1-55 \\ \text { pMB104 } & 140-204 \\ \text { pMB114 } & 56-101 \\ \text { pMB116 } & 1-101 \\ \text { pMB113 } & 89-139 \\ \text { pMB115 } & 89-204 \\ \text { pMB101 } & 1-204 \\ \text { pMB109 } & 1-139 \\ \text { pMB110 } & 56-204 \\ \text { pMB103 } & 56-139\end{array}$
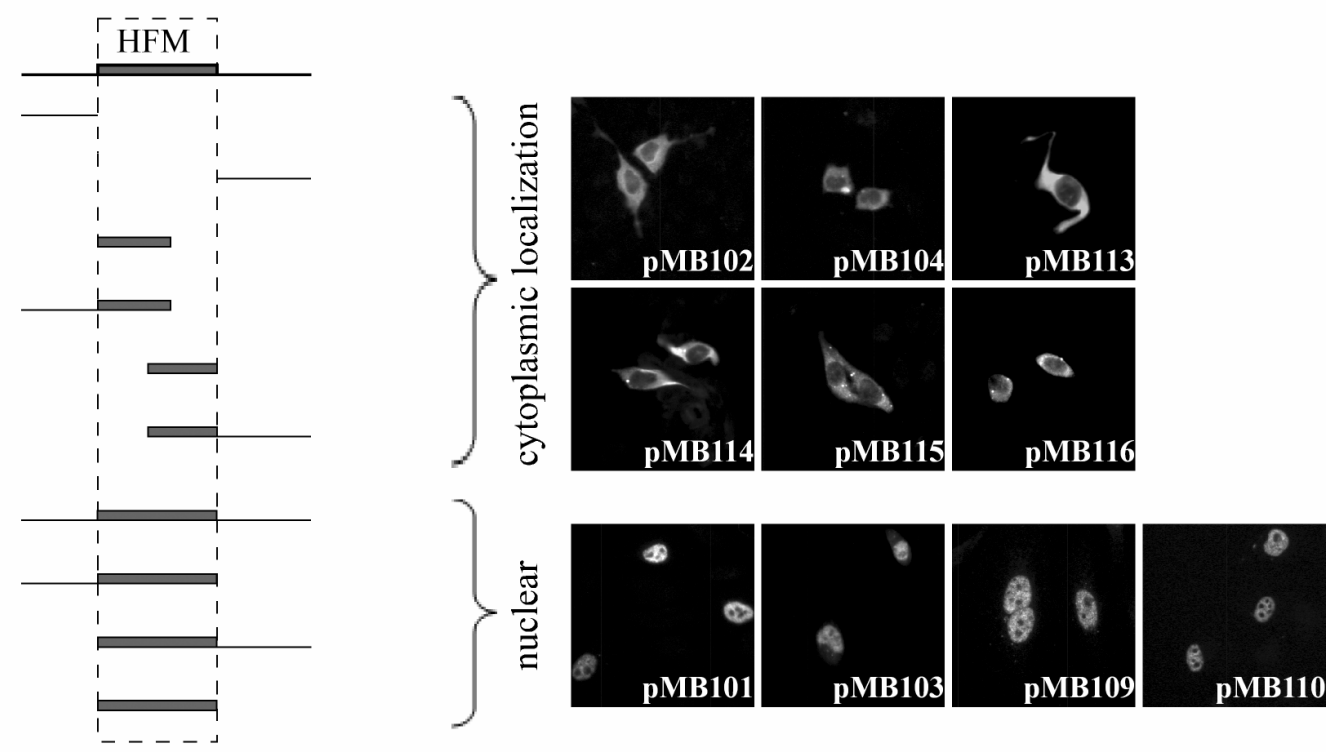

\section{B}

NF-YC-ß-galactosidase-fusions
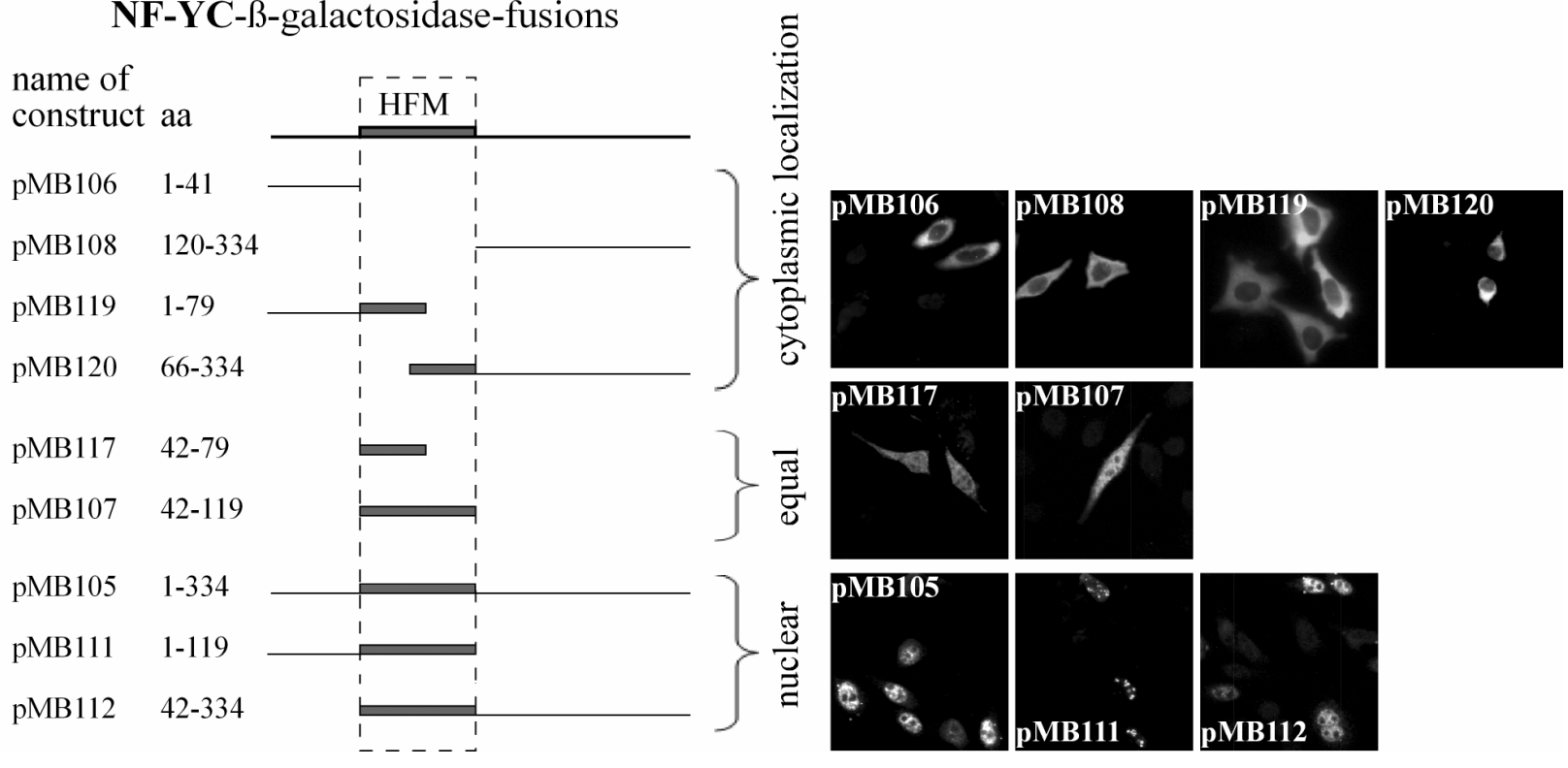

FIG. 6. Nuclear transport of NF-YB and NF-YC fragments fused to B-galactosidase. The regions or sequence motifs that are essential for the nuclear localization of the histone fold motif (HFM) containing subunits were determined by over-expressing fragments of NF-YB (A) and NF-YC (B). HeLa cells were transiently transfected with gene fragments of the subunits fused to bacterial $\beta$-galactosidase as reporter gene and the subcellular localization of the gene products was examined by indirect immunofluorescence 24 hours later. Nuclei were stained with DAPI. On the left, the names of the expression constructs used in this study and the amino acids contained in the constructs are listed. Lines indicate the protein regions and gray bars represent the histone fold motif (HFM) or parts of it. On the right, representative photographs of the subcellular localization of the constructs. The regions required for nuclear localization of NF-YB and NF-YC correspond to the evolutionarily conserved HFM. 
Regarding the regions essential for the nuclear localization of NF-YA, the expression of protein fragments in $\mathrm{HeLa}$ cells revealed that two overlapping regions within the evolutionarily conserved part of NF-YA conferred nuclear import when fused to bacterial $\beta$-galactosidase (Fig.7 lower panel).

NF-YA- $\beta$-galactosidase-fusions

$\begin{array}{ll}\text { name of } & \\ \text { construct } & \text { aa } \\ \text { pJK51 } & 1-181 \\ \text { pJK53 } & 1-261 \\ \text { pJK86 } & \triangle 262-317 \\ \text { pJK79 } & 1-282 \\ \text { pJK88 } & \triangle 282-317 \\ \text { pJK100 } & 1-296 \\ \text { pJK85 } & \triangle 262-297 \\ \text { pJK84 } & \triangle 262-282 \\ \text { pJK56 } & 262-282 \\ \text { pJK57 } & 273-296 \\ \text { pJK67 } & 297-317 \\ \text { pJK78 } & 283-296 \\ & \\ \text { pJK38 } & 1-346 \\ \text { pJK52 } & 182-346 \\ \text { pJK54 } & 262-346 \\ \text { pJK55 } & 262-317 \\ \text { pJK68 } & 262-296 \\ \text { pJK102 } & 283-346 \\ \text { pJK69 } & 283-317 \\ \text { pJK87 } & \triangle 282-297 \\ & \end{array}$
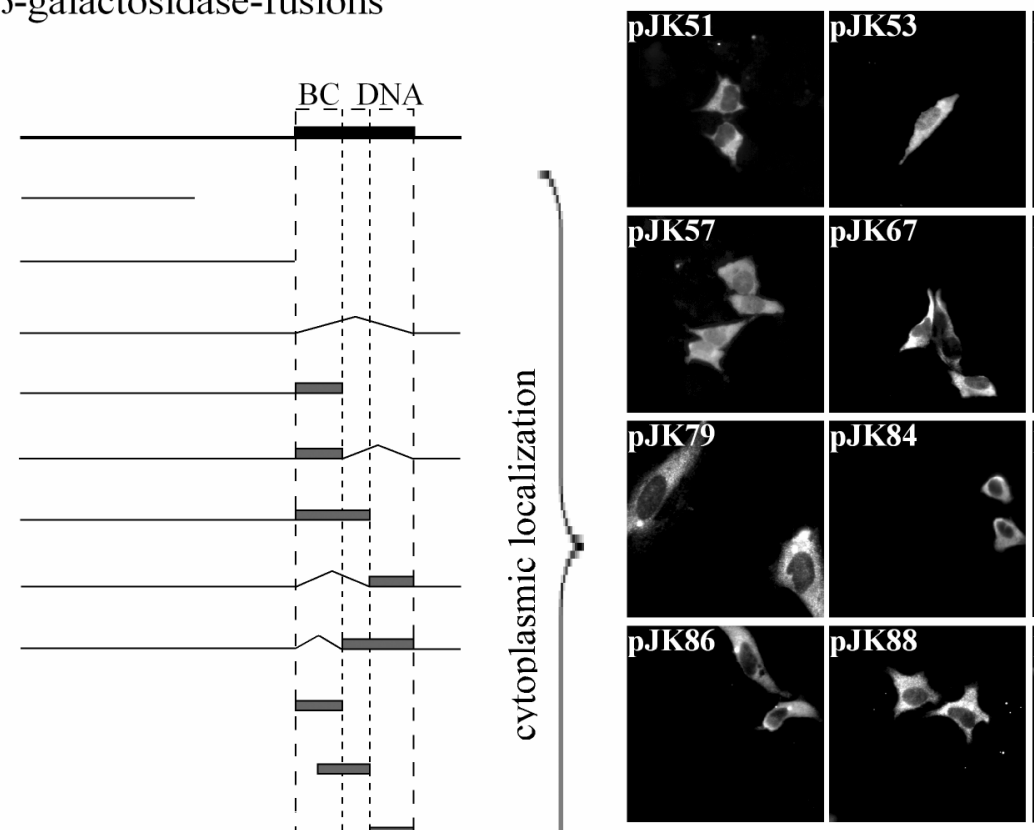

pJK56

pJK51
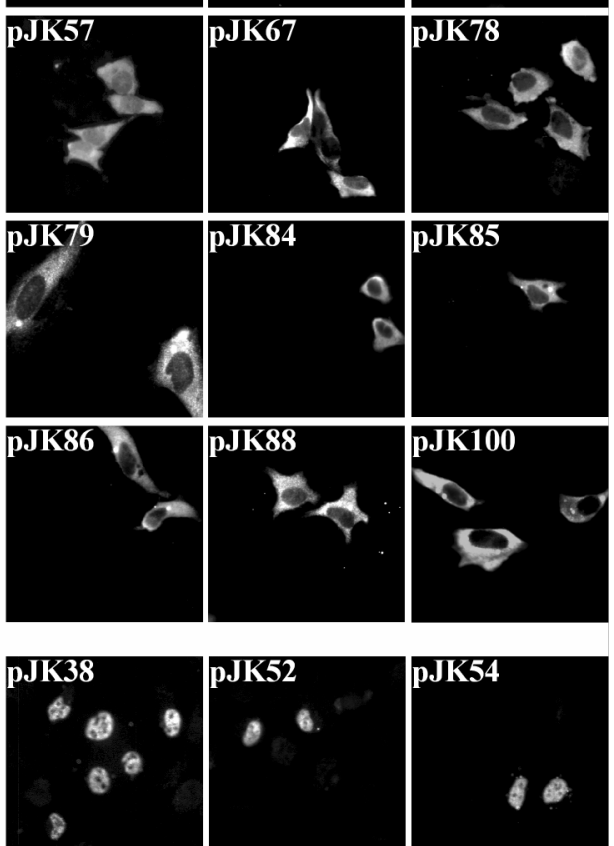

pJK52

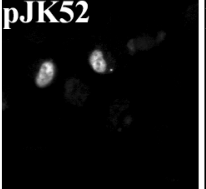

pJK54
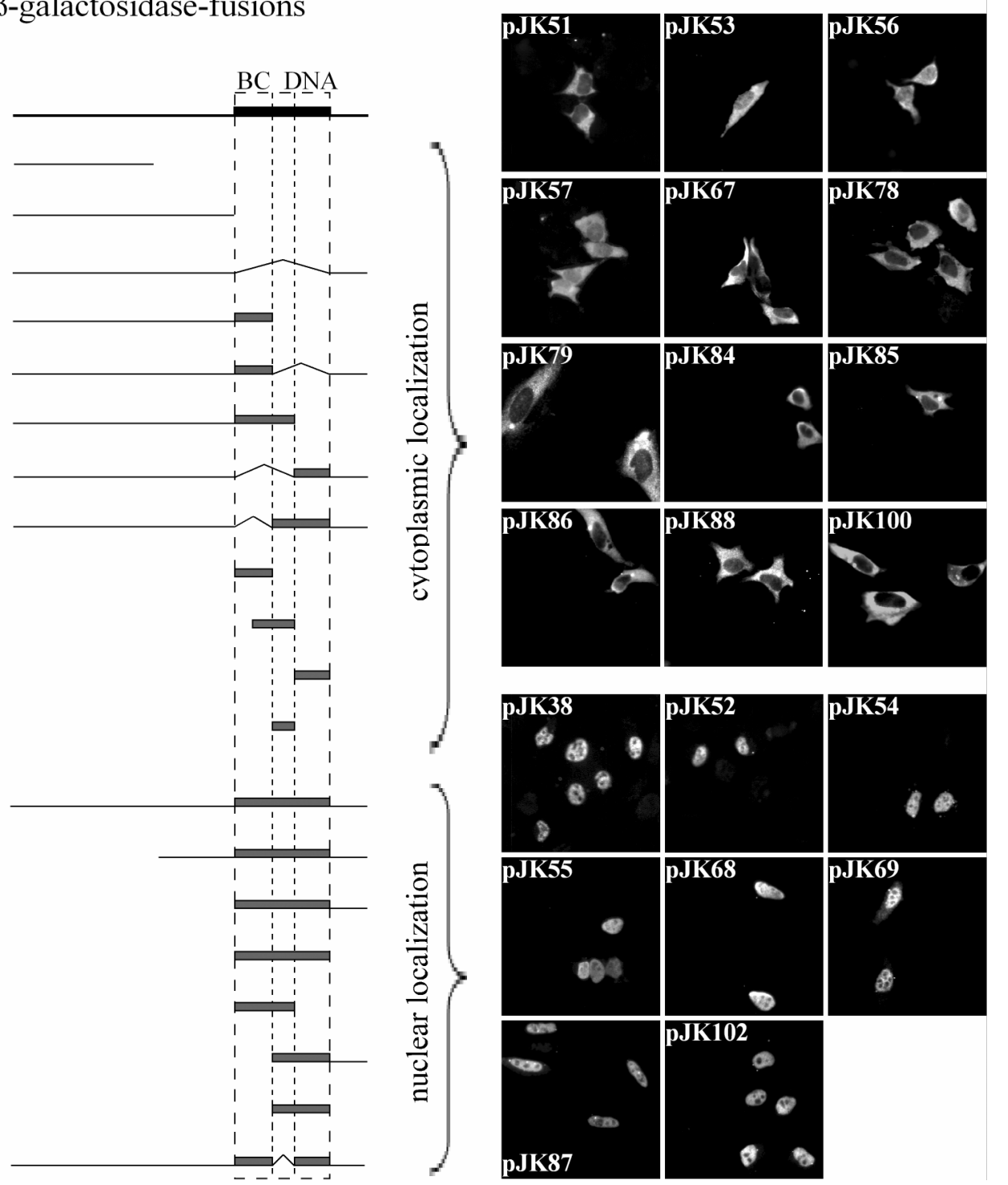

FIG. 7. NF-YA contains a NLS in the evolutionarily conserved domain. The NLS was mapped essentially as described in the legend to FIG. 6. On the left, the names of the expression constructs used in this study and the amino acids contained (or deleted) in the constructs are listed. Lines indicate the protein regions, bent lines indicate deleted regions, and gray bars represent the conserved domain including subunit interaction domain (BC) and DNA binding domain (DNA) or parts of it. On the right, representative photographs of the subcellular localization of the constructs. The 56 amino acid long conserved region at the C-terminus of NF-YA contains a nonclassical NLS. 
In detail, nuclear localization patterns were observed for the linker region attached to either subunit interaction domain (labeled BC) or DNA-binding domain (labeled DNA) when expressed in HeLa cells as $\beta$-galactosidase fusion (Fig.7 lower panel, pJK68 and pJK69). The linker region alone, however, did not confer nuclear localization. Surprisingly, deletion constructs bearing either of these two minimal regions targeting $\beta$-galactosidase into the nucleus were deficient in mediating nuclear uptake (Fig.7 upper panel, pJK84 and pJK100). These results showed that the two minimal regions were not sufficient to confer nuclear import in the context of (nearly) the entire protein.

In conclusion, NF-YA contains a NLS in the 56 amino acid long evolutionarily conserved region at the C-terminus of the protein (amino acids 262-317). This region includes the subunit interaction domain and the DNA-binding domain of NF-YA. The latter is not unexpected since NLS and DNA-binding domain overlap in $90 \%$ of the proteins for which both the NLS and DNA-binding regions are known (Cokol et al., 2000). Since the NLS of NF-YA consists of up to 56 amino acids it does not resemble a short classical NLS (cNLS), but has to be considered as nonclassical NLS (ncNLS) (see also chapter 1.6.1). As a consequence, import receptors probably bind to NF-YA in an importin $\alpha$ (adaptor) independent manner.

\subsubsection{Positively charged amino acids are required for nuclear targeting of NF-YA}

The mapped NLS of NF-YA comprising 11 arginine and seven lysine residues is very similar regarding the number of positively charged amino acids to the BIB domain of rpL23a that contains nine arginine and eight lysine residues (Jakel and Gorlich, 1998). The BIB domain, which might be considered as an archetypical import signal, specifically binds to importin $\beta$, importin 5, importin 7, and transportin (chapter 1.6.1). To analyze the role of the basic amino acid residues of the ncNLS of NF-YA in more detail and to confirm the presence of the NLS in general, we generated mutants randomly substituting basic clusters within the region of interest (Fig.8A) by site-directed mutagenesis using PCR (chapter 2.2.2.16). These mutant constructs along with wild-type NF-YA, all of them fused again to ß-galactosidase as reporter protein, were expressed in HeLa cells and the subcellular localization was detected by indirect immunofluorescence. Based on the fluorescence intensity, the nucleo-cytoplasmic distribution of the over-expressed NF-YA mutants was quantified (using the program ImageJ), and the results of the in vivo transfection studies are summarized in Fig.8B. Regarding the quantitative analysis it should be mentioned that the digital pictures used for quantification are not confocal images and therefore the quantification results include a small (but unavoidable) error due to the cell (cytoplasmic) layer above and beneath the nucleus. 
name of fusion construct ß-gal $\mid$ GST pJK38 pJK95 pJK103 pJK197 pJK104 pJK198 pJK105 pJK199 pJK106 pJK200 pJK130 pJK201 pJK131 pJK202

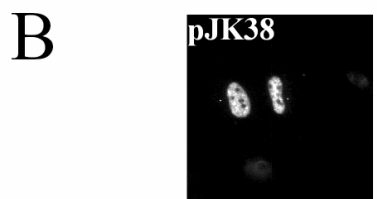

nuclear localization

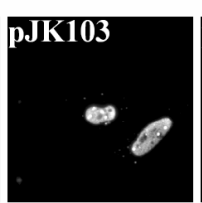

\section{5\%} ( $\mathrm{RSD}=3 \%$ $\mathrm{n}=25$ )

EPLYVNAKQYHRILKRRQARAKLEAEGKIPKERRKYLHESRHRHAMARKRGEGGRF EPLYVNAAQYHRILKRRQARAKLEAEGKIPKERRKYLHESRHRHAMARKRGEGGRF EP LYVNAK QYHR I LNLGQARAKLEAEGKIPKERRKYLHESRHRHAMARKRGEGGRF E P L YVNAK QYHR I LKRRQARAKLEAEGK I PKELGQYLHESRHRHAMARKRGEGGRF EPLYVNAK $Q Y H R I L K R$ RQARAKLEAEGK I PKERRKYLHESRHRHAMALAGGEGGRF E P L YVNAKQYHR I LNLGQARAKLEAEGKI PKELGQYLHESRHRHAMARKRGEGGRF E P L YVNAK QYHR I LNLGQARAKLEAEGK I PKERRKYLHESRHRHAMALAGGEGGRF

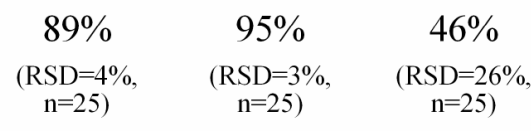

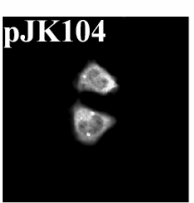

$46 \%$ $\mathrm{n}=25$ )

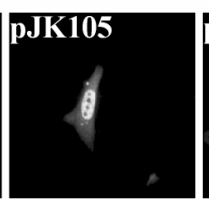

$75 \%$

$(\mathrm{RSD}=19 \%$, $\mathrm{n}=25$ )

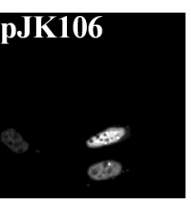

$88 \%$ $(\mathrm{RSD}=7 \%$, $\mathrm{n}=25$ )

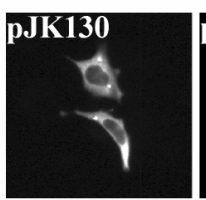

$21 \%$ $(\mathrm{RSD}=29 \%$, $\mathrm{n}=25$ )

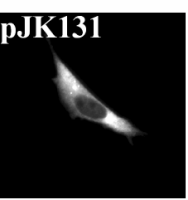

$17 \%$

( $\mathrm{RSD}=24 \%$,

Con beads (pJK): $95 \quad 197198199200201202203$

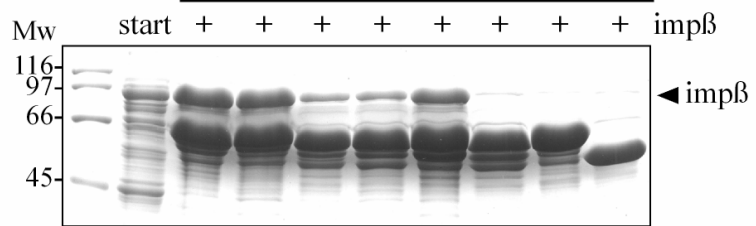

FIG. 8. Characterization of the ncNLS of NF-YA by mutagenesis of basic clusters; positive charge is required for nuclear targeting. (A) Wild-type and mutant NF-YA were fused to either $\beta$-galactosidase or GST (construct names listed on the left). (B) The $ß$-galactosidase-fusion constructs were over-expressed in HeLa cells and the subcellular localization of the NLS mutants was detected by indirect immunofluorescence. The nucleocytoplasmic distribution of the over-expressed NF-YA mutants was quantified with the program ImageJ (nuclear uptake in percent, written below the representative photographs; RSD, relative standard deviation). The results indicate that nuclear import of NF-YA was reduced when the basic cluster (KRR) in the subunit interaction domain (BC) was mutated (pJK104). Mutation of six out of 18 basic amino acids inside the conserved domain (ncNLS) almost completely blocked nuclear uptake (pJK130, pJK131). (C) In addition, binding studies with importin $\beta$ were done using GST fusion constructs carrying the same mutations in the ncNLS of NF-YA as used in the in vivo import experiments described in panel B. The names of these constructs (pJK197-202) are included in panel A. The GSTfusion constructs were expressed in E. coli, immobilized on glutathione-Sepharose, and incubated with importin $\beta$, from bacterial lysate. The bound fractions were analyzed by SDS-PAGE followed by Coomassie staining. The basic cluster (KRR) in the subunit interaction domain proved to be crucial for importin ( $\beta$ ) binding and the additional mutation of three more positively charged amino acid residues caused a complete loss of importin $\beta$ binding (pJK201, pJK202). The gene product pJK203 (1-269aa) which resembles a C-terminal NF-YA-deletion construct lacking nearly the entire conserved domain (harboring the ncNLS) was used as negative binding control (DNA, DNA binding domain; $Q$, glutamine-rich trans-activation domain). The importin $\beta$ binding data are discussed in detail below (chapter 3.1.2.3). 
Nuclear localization of NF-YA is strongly reduced when the basic cluster (KRR) in the subunit interaction domain (BC) was mutated (pJK104, $46 \%$ nuclear uptake corresponding to an equal distribution of fluorescence in nucleus and cytoplasm). In contrast, the mutation of other positively charged amino acid residues and basic clusters did not (pJK103, pJK106) or just moderately (pJK105) reduce the nuclear localization of (mutant) NF-YA (Fig.8B). However, mutation of the three basic amino acid residues (KRR) in the subunit interaction domain in addition to the mutations as described in pJK105 or pJK106, respectively (six out of the 18 positively charged amino acid residues mutated in total), led to a significant loss of nuclear accumulation (Fig.8B, pJK130: $21 \%$ and pJK131: $17 \%$ nuclear localization).

Two conclusions can be drawn from these observations: Firstly, a certain number of positively charged amino acid residues in the NLS sequence of NF-YA is required for nuclear uptake. Secondly, some of the basic amino acid residues in the region essential for subunit interactions (Mantovani et al., 1994) are also crucial for nuclear localization (for example amino acids mutated in pJK104, Fig.4A). Because of the dual function of these amino acid residues (nuclear localization and subunit interaction) we later addressed the question whether the ncNLS in NF-YA can be further recognized by import factors once the subunit is assembled into the NF-Y complex.

Additionally, the mutant NF-YA constructs were expressed as GST-fusion proteins in E. coli and tested for binding to importin $\beta$ (Fig.8C). The results of these in vitro binding studies are described and discussed below in detail (in the context of the identification of importin $\beta$ as import receptor for NF-YA, see chapter 3.1.2.3).

\subsubsection{Active nuclear transport of NF-Y subunits in Xenopus oocytes}

After identification of the minimal nuclear targeting signals in NF-YA, NF-YB, and NF-YC we next wanted to characterize and compare the nuclear transport of the three different NF-Y subunits. For that reason, myc-tagged fusion constructs of the individual NF-Y subunits were in vitro transcribed and translated using the $\mathrm{T}_{\mathrm{N}} \mathrm{T}$ coupled reticulocyte lysate system (chapter 2.2.8). Using this method, the fusion proteins were also ${ }^{35} \mathrm{~S}$-labeled, which allowed detection of the different proteins by phosphorimaging. The in vitro transcribed and translated NF-Y subunits were then injected into the cytoplasm of Xenopus oocytes. After microinjection, nuclear and cytoplasmic fractions of the injected oocytes were separated manually at the indicated time points and homogenized. The fusion proteins were then immunoprecipitated using antibodies against the myc-tag and analyzed by SDS-PAGE with subsequent phosphorimaging (see also chapter 2.2.9). 
As shown in Fig.9A, 24 hours after microinjection the majority (about $80 \%$ ) of the cytoplasmically injected NF-Y subunits were detected in the nuclear fractions. However, the kinetics of nuclear translocation differed among the three NF-Y subunits. While NF-YA was imported rapidly into the nucleus of Xenopus oocytes, NF-YB and NF-YC were imported much slower (for better illustration see graphic in Fig.9B). One explanation for these different import kinetics would be that the interaction with the endogenous histone fold partner, which is abundant in Xenopus oocytes ( $\mathrm{Li}$ et al., 1998), is a prerequisite for nuclear uptake. When the temperature was lowered to $4^{\circ} \mathrm{C}$ the nuclear import of the three subunits was nearly blocked (Fig.9C). This strong temperature dependence points to an active (energy-dependent) nuclear transport of the NF-Y subunits rather than passive diffusion. This is due to the fact that diffusion processes depend on the absolute (but not relative) temperature. Hence, when passive diffusion would have caused the nuclear accumulation of NF-Y subunits a temperature drop of $14^{\circ}$ Kelvin should have had almost no effect on the nucleocytoplasmic distribution pattern. This however was not the case since nuclear uptake of NF-Y subunits was strongly diminished at $4^{\circ} \mathrm{C}$. It should be mentioned that, for the microinjection experiments presented in Fig.9 (panel A and C) the same batch of Xenopus oocytes was used. Since the nuclei of the oocytes allowed nuclear uptake of the three different in vitro transcribed and translated NF-Y fusion proteins, but restricted GST (injection and separation control) to the cytoplasm, in addition to the fact that lower temperature is very unlikely to damage the cell nucleus, no further control for nuclear integrity was used.

Furthermore, while nuclear uptake of microinjected NF-YA was independent of the quantity of injected protein, the relative amount of nuclear import of NF-YB and NF-YC depended on the quantity of injected protein. When twice the amount of NF-YB and NF-YC was injected compared to the conditions applied in Fig.9A (40 nl instead of $20 \mathrm{nl}$ ) the percentage of nuclear uptake of NF-YB and NF-YC already dropped significantly (Fig.9F). In general, it could be observed that the more protein was injected the lower was the percentage of nuclear uptake. Although receptor-mediated transport processes are saturable in relation to the cargo, in this case it rather suggests that the amount of endogenous corresponding histone fold partner limits the nuclear transport capacity. These results are in accordance with the differences observed between strong and moderate over-expression of NF-YB and NF-YC in HeLa cells (chapter 3.1.1.1) and further indicate that nuclear accumulation presumably relies on interaction/dimerization of the two HFM containing subunits. 
A
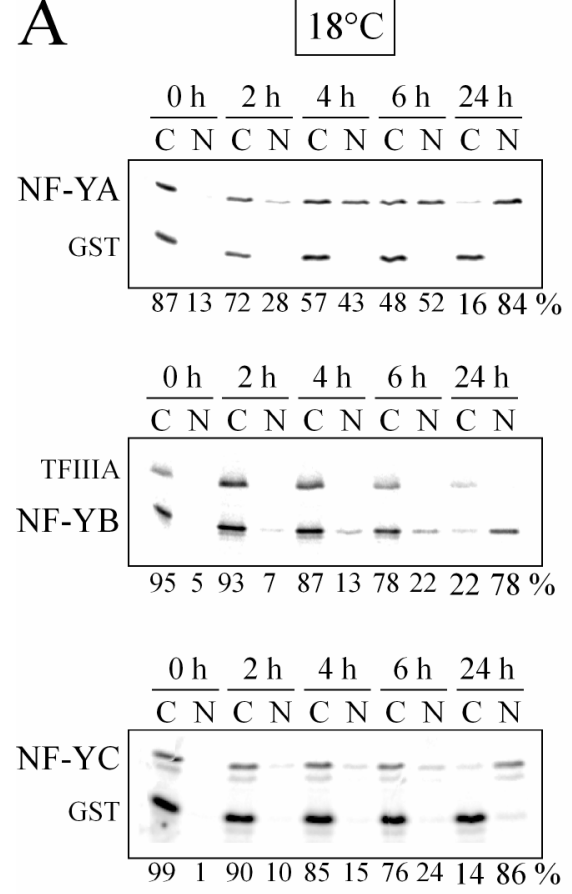

$\mathrm{D}$

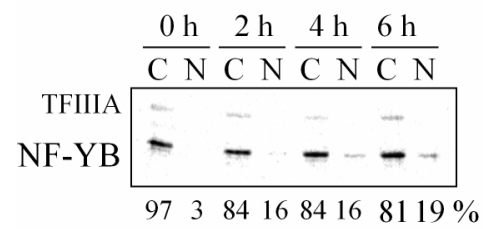

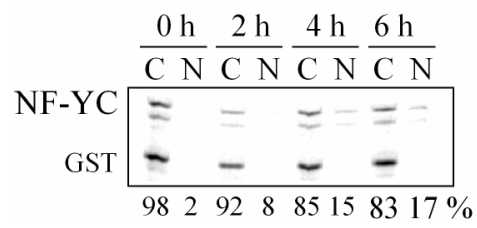

B

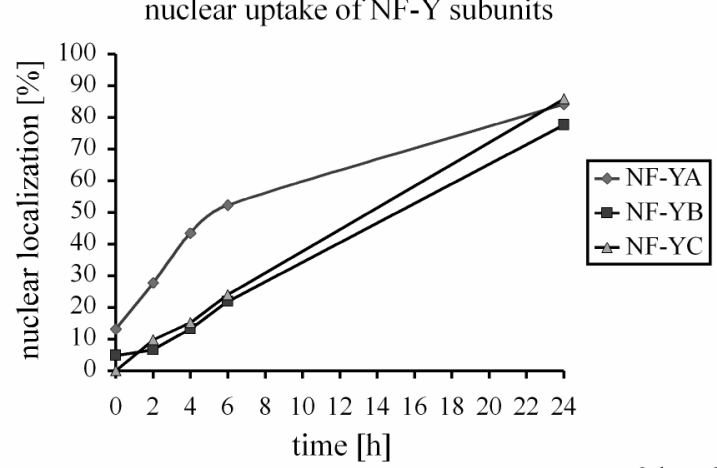

C time $[\mathrm{h}]$
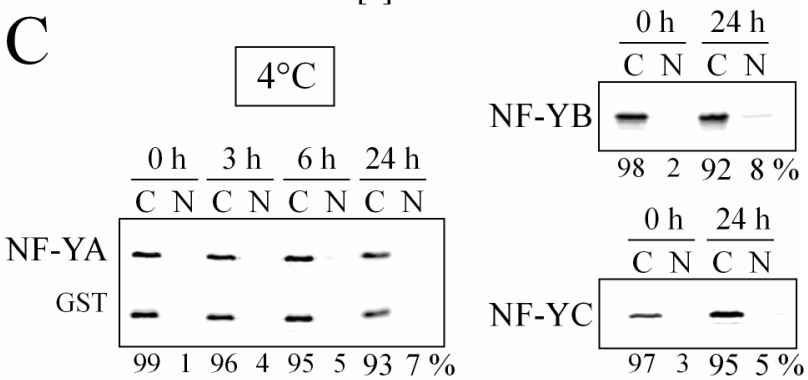

$\mathrm{F}$

double amount injected $(40 \mathrm{nl})$

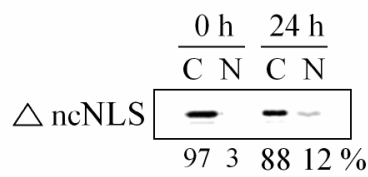

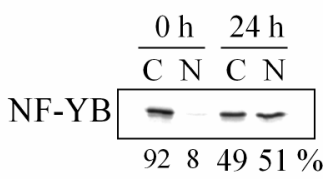

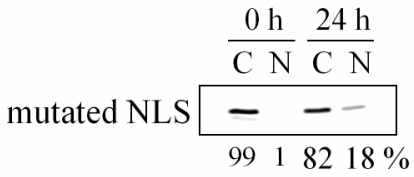

FIG. 9. Active nuclear transport of NF-Y subunits in Xenopus laevis oocytes. ${ }^{35} \mathrm{~S}$-labeled NF-YA, NF-YB, NF-YC myc-tagged fusion proteins were injected into the cytoplasm of Xenopus oocytes; nuclear (N) and cytoplasmic (C) fractions were separated manually, either immediately $(0 \mathrm{~h})$ or at the indicated time points, and were analyzed for their nucleocytoplasmic distribution by SDS-PAGE. To control the injection/separation of nuclear and cytoplasmic fractions either transcription factor IIIA (TFIIIA) or GST protein were co-injected. The nucleocytoplasmic distribution of the injected proteins was quantified (in percent, written below the gels). (A) 24 hours after microinjection the majority of the cytoplasmic injected NF-Y subunits were detected in the nuclear fractions. (B) The kinetics of nuclear translocation differ among the three subunits, NF-YA was imported rapidly while the histone fold motif (HFM) subunits NF-YB and NF-YC in comparison were imported much slower. (C) When the temperature was lowered to $4^{\circ} \mathrm{C}$ the nuclear import of the subunits was blocked. (D) Co-injection of antiNF-YA antibodies had no significant influence on the nucleocytoplasmic distribution of the HFM containing subunits (compare with panel A) indicating that the nuclear import of NF-YB and NF-YC is NF-YA independent. (E) 24 hours after microinjection the majority of the cytoplasmically injected NF-YA mutants [ $\triangle$ ncNLS represents myc-tagged pJK86 (FIG. 7); mutated NLS resembles myc-tagged pJK130 (FIG. 8A)] were detected in the 
cytoplasmic fractions. (F) When twice the amount ( $40 \mathrm{nl})$ of NF-YB and NF-YC were injected (in comparison to the conditions applied in panel A) the import rates of NF-YB and NF-YC dropped significantly.

To investigate a possible role of NF-YA regarding the nuclear import of NF-YB and NF-YC, anti-NF-YA antibodies were co-injected into the cytoplasm of oocytes. As shown in Fig.9D (for comparison see Fig.9A again), co-injection of anti-NF-YA antibodies had no significant influence on the nucleocytoplasmic distribution of the two HFM containing subunits. Six hours after microinjection the amount of NF-YB and NF-YC in the nuclear fractions were only slightly lower than without co-injecting anti-NF-YA antibodies indicating that nuclear import of NF-YB and NF-YC is NF-YA independent. These results are again in line with the previous observations that co-expression of the corresponding histone fold partner was sufficient to mediate nuclear localization of strongly over-expressed HFM containing subunits in HeLa cells. In those experiments the additional co-expression of NF-YA was not necessary to achieve nuclear accumulation.

Finally, also the previously characterized NF-YA mutants ( $\triangle$ ncNLS resembles pJK86 from Fig.7 and mutated NLS resembles pJK130 from Fig.8A, but myc- instead of $\beta$-gal-tagged) were cytoplasmically injected to see whether the results of the in vivo transfection experiments could be reproduced. As shown in Fig.9E, 24 hours after microinjection the majority of the injected dominant negative NF-YA mutants were still detected in the cytoplasmic fractions. This further confirmed that NF-YA contains a NLS in the 56 amino acid long evolutionarily conserved region at the $\mathrm{C}$-terminus of the protein, and that a certain number of positively charged amino acid residues in the NLS sequence of NF-YA is required for nuclear uptake.

\subsubsection{In vitro studies}

\subsubsection{Characteristics of a receptor-mediated transport process}

So far, the subcellular localization of NF-Y subunits was analyzed, the minimal regions essential for the nuclear localization were mapped, and the nuclear import mechanism was kinetically characterized. Next, transport receptors involved in the nuclear import of NF-YA, NF-YB, and NF-YC had to be identified by in vitro binding studies and in vitro nuclear import assays. The in vitro import assay based on the method established by Adam et al. (Adam et al., 1990) eventually allows the reconstitution of nuclear import events by using recombinant purified transport factors without the interference of endogenous import factors. Using this method, we first tried to further characterize the nuclear transport process of the three NF-Y subunits in vitro (without the endogenous background). For that purpose, HeLa cells were grown on glass 
coverslips, plasma membranes were selectively permeabilized with digitonin, and either rabbit reticulocyte lysate as general source of exogenous import receptors or purified recombinant import factors were used to reconstitute nuclear import events. The permeabilized cells were incubated in a humidity chamber with fluorescently labeled NF-Y subunits (cargo proteins) and reticulocyte lysate along with an energy-regenerating system (see also chapter 2.2.6). Recombinant purified proteins gain intracellular access when overlaid on the digitoninpermeabilized cells and subsequent nuclear import causes nuclear staining. The nuclei were visualized with the DNA-binding substrate DAPI. The cargo proteins were tagged (6z, EGFP, or GST) which increased their molecular size to avoid passive diffusion. The exclusion size of the nuclear pore complexes in permeabilized HeLa cells is about $50 \mathrm{kDa}$. For the initial in vitro experiments to characterize the nuclear transport processes of NF-YA, NF-YB, and NF-YC the three NF-Y subunits carried a $6 \mathrm{z}(\sim 42 \mathrm{kDa})$ tag (chapter 2.2.2.15) and were fluorescently labeled (chapter 2.2.5). The results of these in vitro nuclear transport studies are summarized in Fig.10.

Although nuclear staining of fluorescently labeled NF-YA could already be observed in the absence of transport receptors, import of NF-YA was strongly stimulated by the addition of reticulocyte lysate (Fig.10A). In contrast, the presence of import receptors in the form of reticulocyte lysate did not support nuclear accumulation of the monomeric HFM containing subunits NF-YB and NF-YC (Fig.10C). Instead, these two subunits remained in the cytoplasm in close vicinity to the nuclear membranes as indicated by the rim staining. In general, rim staining only occurs when importin-cargo complexes are not dissociated, respectively are not released from nucleoporins at the nuclear basket. However, in this particular case it presumably resembles an artificial behavior (perhaps caused by the fluorescence labeling) rather than a physiologically relevant feature of NF-YB and NF-YC. The foregoing in vivo studies had strongly suggested that NF-YB and NF-YC mediate the nuclear localization of its corresponding histone fold partner, or rather the nuclear accumulation of NF-YB and NF-YC appeared to rely on this interaction. Thus, the cytoplasmic localization of the monomeric HFM containing subunits in these in vitro import assays (even in the presence of import receptors) was not surprising, but rather expected. The endogenous amounts of NF-YB and NF-YC in reticulocyte lysate (not determined) obviously were insufficient for mediating the nuclear uptake of recombinant, purified, 6z-tagged and fluorescently labeled NF-YB and NF-YC.

In contrast to the HFM containing subunits NF-YB and NF-YC, the in vitro studies regarding the nuclear accumulation of NF-YA in the presence of reticulocyte lysate were further extended. 
A import of fluorescent 6z-NF-YA with energy, reticulocyte lysate and:

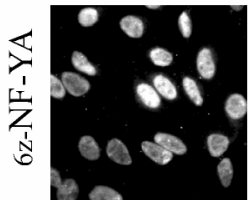

w/o retic

$4^{\circ} \mathrm{C}$

w/o energy

WGA RanQ69LGTP
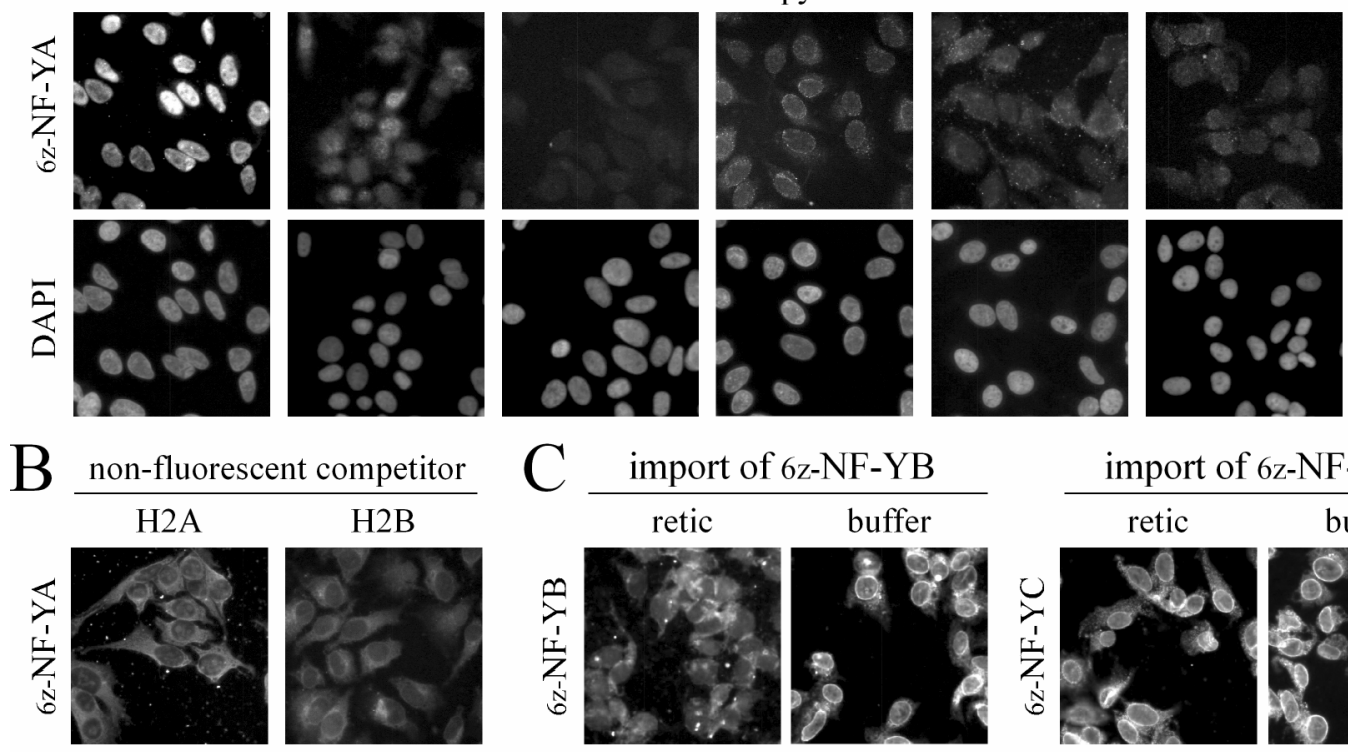

$C \frac{\text { import of } 6 z-N F-Y B}{\text { retic } \quad \text { buffer }}$

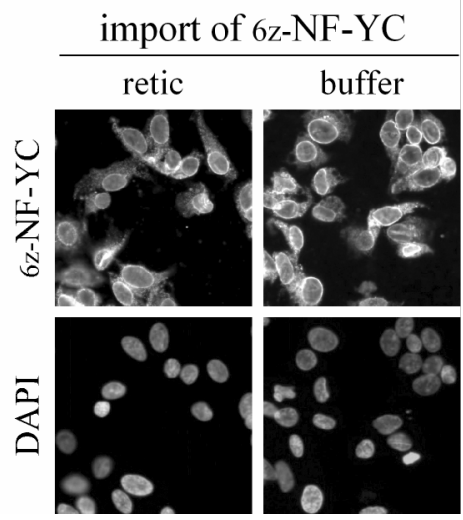

FIG. 10. Nuclear import of NF-YA shows characteristics of a receptor-mediated process while monomeric HFM containing subunits are not transported into the nuclei of permeabilized cells.

Digitonin-permeabilized HeLa cells were incubated with about $1 \mu \mathrm{M}$ of cargo substrate, rabbit reticulocyte lysate (retic) and an energy regenerating system (energy) for $25 \mathrm{~min}$ at $37^{\circ} \mathrm{C}$ (see also materials and methods). For a negative control reticulocyte lysate was replaced by transport buffer (buffer or w/o retic). Substrates were labeled with a fluorescein derivative and were detected by direct immunofluorescence after fixation. (A) In vitro nuclear import reactions with 6z-NF-YA were carried out in the presence and absence of reticulocyte lysate at $37^{\circ} \mathrm{C}$, at $4{ }^{\circ} \mathrm{C}$, in the presence of $100 \mathrm{U} / \mathrm{ml}$ apyrase (omitting the energy), WGA (100 $\mu \mathrm{g} / \mathrm{ml})$, and RanQ69L-GTP (15 $\mu \mathrm{M})$. Transport analysis under these different inhibitory conditions showed that the nuclear transport of 6z-NF-YA is temperature-, energy- and Ran-dependent, and requires access to nucleoporins. Note, that the 6z-NF-YA fusion protein tended to passively diffuse into the nucleus of permeabilized cells since nuclear fluorescence in the absence of import receptors was observed (w/o retic). (B) NF-YA import is competed by core histones (e.g. H2A, H2B). Nuclear import reactions were performed in the presence of retic and energy and were supplemented with either $10 \mu \mathrm{M} \mathrm{H} 2 \mathrm{~A}$ or $\mathrm{H} 2 \mathrm{~B}$ as indicated. Both core histones completely inhibit the nuclear uptake of NF-YA. (C) 6z-NF-YB and 6z-NF-YC were not imported into nuclei of digitonin-permeabilized cells in the presence of retic and energy, but remained in the cytoplasm in close vicinity to the nuclear membrane.

Firstly, the effects of known inhibitors of importin-mediated nuclear transport processes were analyzed. In detail, in vitro nuclear import reactions with NF-YA were carried out at lower temperature $\left(4^{\circ} \mathrm{C}\right)$, and in the presence of apyrase, wheat germ agglutinin (WGA), and 
RanQ69L-GTP (Fig.10A). Import of NF-YA was indeed sensitive to all of the applied inhibitors as indicated by reduction of nuclear fluorescence. Keep in mind, when NF-YA (or a transport cargo in general) was not imported in the nucleus and then was washed out from the cytoplasm neither nuclear nor cytoplasmic staining could be observed. In detail, nuclear localization of NF-YA was blocked both at low temperature and by apyrase treatment (depletion of endogenous ATP) indicating that NF-YA accumulates in the nuclei of permeabilized cells by a signalmediated import rather than by passive diffusion. Nuclear fluorescence was also abolished when the permeabilized cells were incubated with WGA. This lectin binds specifically to glycosylated residues of several nucleoporins and therefore inhibits the receptor-mediated translocation of cargo molecules through the nuclear pore complexes (Finlay et al., 1987), but still allows passive diffusion (Palacios et al., 1997). Thereby it is not known whether the blocked nuclear transport is caused by competition of WGA with import receptors or by steric interference. Since addition of WGA markedly inhibited the nuclear uptake of NF-YA, access to the nucleoporins must be a prerequisite for its nuclear accumulation. Finally, if the interaction between NF-YA and importins was required for nuclear entry, in vitro import reactions should also be RanGTPsensitive. Again nuclear uptake of NF-YA into the nuclei of permeabilized cells was sensitive to the addition of RanQ69L(GTP), a Ran point mutant which is unable to hydrolyze GTP, suggesting that members of the importin $\beta$ family mediate NF-YA import. Taking the results of the inhibition experiments together, it can be concluded that the nuclear import of NF-YA meets the criteria of a signal-dependent, energy-dependent, receptor-mediated process.

To characterize the pathway involved in the nuclear import of NF-YA even more specifically, we performed the in vitro import assay in the presence of rabbit reticulocyte lysate and nonfluorescent core histones as potential import competitors. The addition of individual nonfluorescent core histones to the import mix completely blocked the nuclear import of NF-YA (shown for H2A and H2B in Fig.10B). The competition by core histones indicates that NF-YA uses the same pathway for nuclear entry as the core histones. In other words, the nuclear import of NF-YA depends entirely on at least one of the import receptors that mediate nuclear transport of the core histones, namely importin $\beta$, importin 5 , importin 7 , importin 9 or transportin (Baake et al., 2001a; Muhlhausser et al., 2001).

\subsubsection{NF-Y subunits interact with different import receptors}

The results described above suggested that NF-Y subunits are actively imported into the nucleus. As a consequence, nuclear transport of NF-YA, NF-YB, and NF-YC was most likely to be mediated by transport receptors of the importin $\beta$ superfamily (see also chapter 1.4). The 
identification of the mediators of nuclear uptake of the different subunits was then done by in vitro binding studies using recombinant importins. As mentioned above, the mapped NLS of NF-YA resembles rather a nonclassical (importin $\alpha$-independent) arginine rich type of signal similar to the BIB domain of the ribosomal protein L23a. The BIB domain confers import by importin $\beta$, importin 5, importin 7, and transportin. Therefore it was rational to investigate first the role of these four import receptors regarding the nuclear transport of NF-YA. In addition, importin 13 was included since binding of the NF-YB like protein YBL1 (Bolognese et al., 2000) to immobilized importin 13 had been reported by Mingot et al. (Mingot et al., 2001). YBL1 shows a strong NF-YB homology covering the histone fold motif, but despite their similarity YBL1 differs from NF-YB in respect to specific functions (Bolognese et al., 2000). In order to identify potential import receptors, GST pulldown assays (chapter 2.2.4.1) were performed with the different NF-Y subunits (Fig.11).

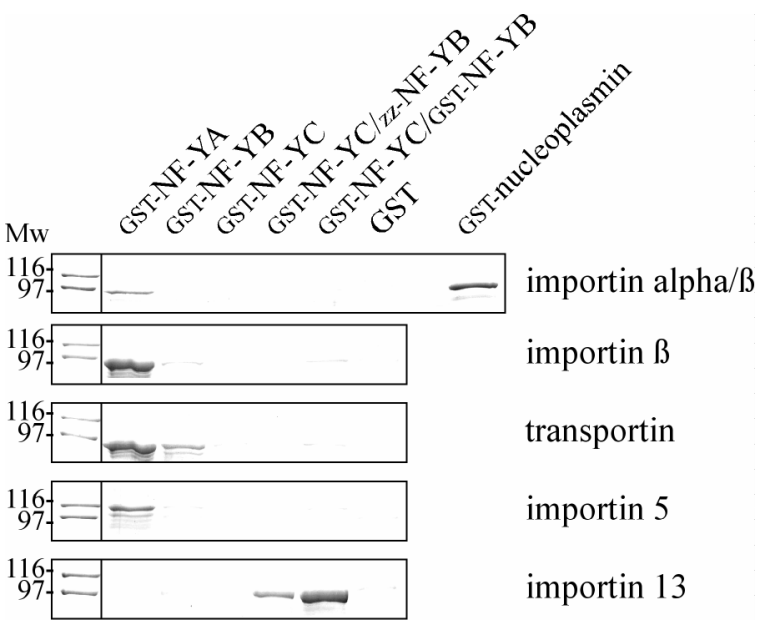

FIG. 11. Interaction of NF-Y subunits with different import receptors. The three NF-Y subunits were expressed as GST fusions in E. coli and immobilized on glutathione-Sepharose. In addition, the HFM containing subunits were co-expressed in E. coli as GST-NF-YB/GST-NF-YC complex and zZ-NF-YB/GST-NF-YC complex and used as bait after immobilization on glutathione-Sepharose. The immobilized fusion proteins were incubated with either importin $\alpha / \beta$, importin $\beta$, transportin, importin 5 or importin 13, all from bacterial lysates. The bound fractions were analyzed by SDS-PAGE followed by Coomassie staining. Note, importin $\beta$, transportin, and importin 5 were bound to NF-YA whereas under the same conditions none of the used import factors bound efficiently to single HFM containing subunits NF-YB and NF-YC. Surprisingly, importin 13 only bound the dimerized NF-YB/NF-YC complex while binding of other transport receptors to the pre-formed HMF-dimer was insignificant. Further, the importin $\alpha / \beta$ heterodimer just bound efficiently to the control cargo nucleoplasmin and importins did not bind to GST alone. 
In detail, the three NF-Y subunits were expressed as GST fusions in E. coli and immobilized on glutathione-Sepharose beads. In addition, the HFM containing subunits were co-expressed in E. coli as either GST-NF-YB/GST-NF-YC or zz-NF-YB/GST-NF-YC complex and also used as bait after immobilization on glutathione-Sepharose beads (for expression and purification of NF-Y subunits, see also chapter 2.2.3.3.5.1). It had to be kept in mind that GST can dimerize and thus unintended interactions between two GST fusion proteins can take place. Therefore, the HFM containing subunits were additionally co-expressed as zz/GST fusion proteins to entirely exclude this possibility and to assure dimerization of NF-YB and NF-YC via their histone fold motifs.

The pre-loaded beads were then incubated with an importin $\alpha / \beta$ heterodimer, importin $\beta$, transportin, importin 5, and importin 13, all from bacterial lysates (chapter 2.2.3.3.6.2), and were subsequently washed. The retained proteins were analyzed by SDS-PAGE followed by Coomassie staining. Importin $\beta$, transportin, and importin 5 showed binding to NF-YA whereas under the same conditions none of the used import factors bound efficiently to the monomeric NF-YB and NF-YC subunits. Surprisingly, importin 13 only bound to the NF-YB/YC dimer while binding of other transport receptors to the preformed complex was insignificant (Fig.11).

\subsubsection{Importins bind specifically to NF-Y subunits}

In order to verify that binding of the different members of the importin $\beta$-family to NF-YA and binding of importin 13 to the NF-YB/YC complex is specific, binding studies were done in the presence and absence of RanGTP which has been used to simulate nuclear Ran conditions. As previously mentioned (chapter 1.5), importins load cargoes in the absence of RanGTP in the cytoplasm and release their cargo upon RanGTP binding in the nucleus (Gorlich et al., 1996b; Izaurralde et al., 1997; Rexach and Blobel, 1995). Hence, specific binding of cargo proteins to import receptors is strongly reduced in the presence of RanGTP.

Immobilized GST-NF-YA was incubated with purified recombinant importin $\beta$, importin 7 , transportin, and importin 5 (Fig.12A). All the import factors used were bound specifically to NF-YA and this binding was reduced by the GTP-bound form of RanQ69L. Among the four import factors that bound specifically to NF-YA importin $\beta$ showed the highest binding competence (Fig.12B). On the other hand the immobilized NF-YB/NF-YC complex (again as either GST-NF-YB/GST-NF-YC or zz-NF-YB/GST-NF-YC) showed binding of recombinant importin 13 from a bacterial lysate. The retained proteins were analyzed by SDS-PAGE and indeed importin 13 was bound specifically to the histone fold dimer and this binding was abolished by RanGTP, as expected for an import cargo (Fig.12C). 


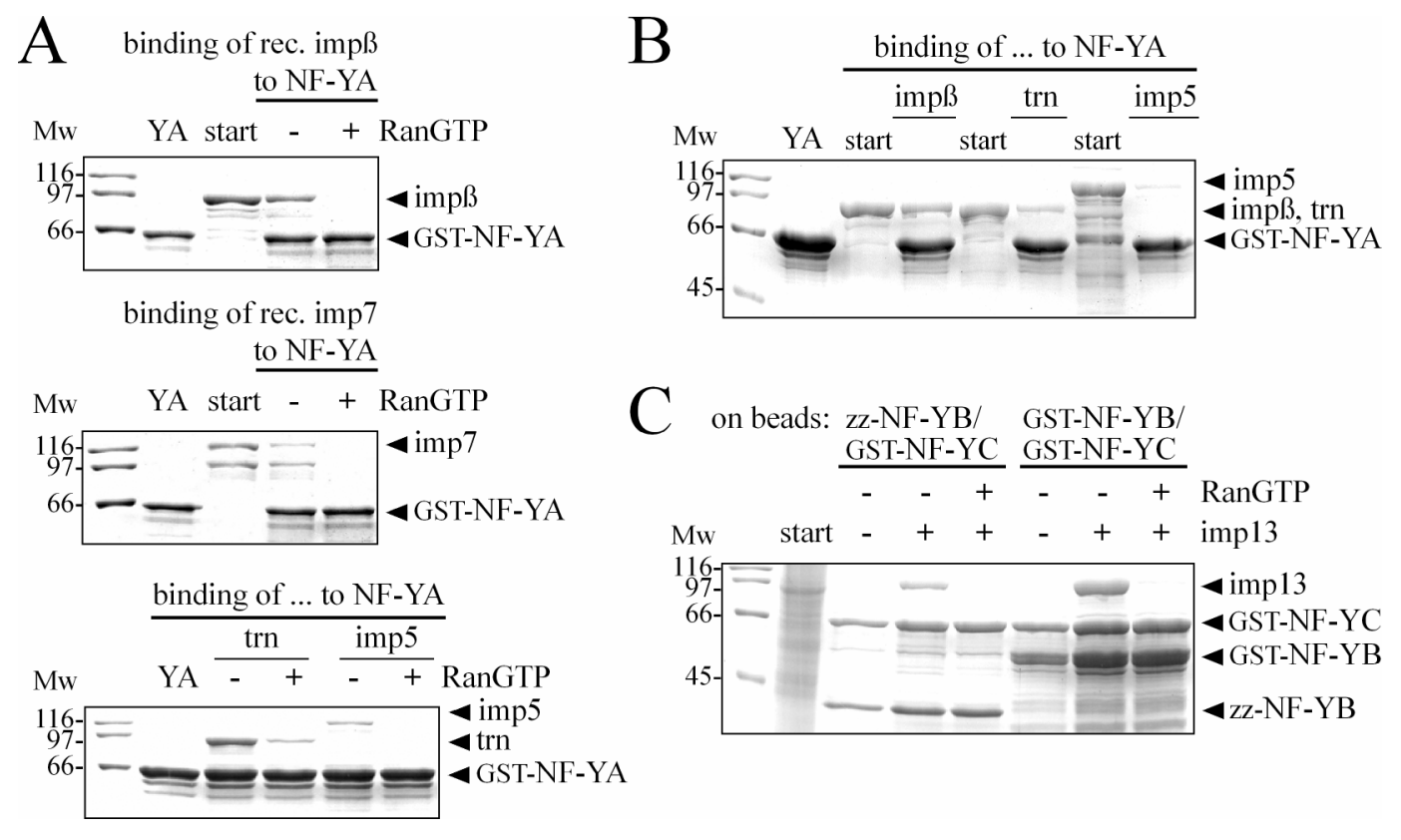

FIG. 12. Specific binding of importins to NF-Y subunits. GST pulldown assays were performed as described in the legend to FIG. 11. (A) Immobilized GST-NF-YA was incubated with purified recombinant importin $\beta$, importin 7 , transportin, and importin 5 . Starting material (10\% of the importins that were used) and bound fractions were analyzed by SDS-PAGE followed by Coomassie staining. As shown in panel A the import receptors were bound specifically to immobilized NF-YA and this binding was reduced by the GTP-bound form of RanQ69L $(2 \mu \mathrm{M})$ which was used to simulate nuclear Ran conditions. (B) Among the transport factors binding to NF-YA importin $\beta$ showed the highest binding competence. (C) Immobilized zz-NF-YB/GST-NF-YC complex and GST-NF-YB/GST-NF-YC complex (see also arrows on the right) were used to bind recombinant importin 13 from a bacterial lysate. Importin 13 was bound specifically to the immobilized NF-YB/NF-YC dimer and this binding was abolished by RanGTP. Hence, the NF-YB/NF-YC dimer behaves as an importin 13-specific import substrate. GST alone did not bind to import receptors (see again FIG. 11).

Since importin $\beta$ had shown the highest binding competence to GST-NF-YA these in vitro binding studies were extended to the different NLS mutants characterized in Fig.8A and Fig.8B (chapter 3.1.1.4) The mutant NF-YA GST-fusion constructs were expressed in E. coli, immobilized on glutathione-Sepharose, incubated with importin $\beta$ (from bacterial lysate), and bound fractions were analyzed by SDS-PAGE followed by Coomassie staining. These data are included in Fig.8C (chapter 3.1.1.4). The results of the in vitro binding assays indicate that the basic clusters in the subunit interaction domain (KRR, pJK198) and linker region (RRK, pJK199) are crucial for importin $\beta$ binding. The additional mutation of three more positively charged amino acid residues caused a complete loss of importin $\beta$ binding (pJK201, pJK202). These binding data fit well with the results from the in vivo transfection studies (Fig.8B) and 
strongly support our conclusion that a certain number of positively charged amino acid residues in the NLS sequence of NF-YA are required for nuclear targeting.

In conclusion, NF-YA contains an ncNLS corresponding to the evolutionarily conserved domain at the $\mathrm{C}$-terminus of the protein which confers binding of importin $\beta$, importin 5 , importin 7 , and transportin. Further, nuclear uptake of NF-YA into the nucleus of digitonin-permeabilized cells could be completely blocked by an excess of core histones. This competition indicated that at least one of the five import factors that mediate nuclear import of the core histones is required for nuclear accumulation of NF-YA. While four of them showed specific binding to NF-YA the possible role of importin 9 (not in hands) has still to be elucidated. Regarding the nuclear transport of NF-YB and NF-YC in vivo experiments indicated that the HFM containing subunits mediate the nuclear localization of each other. Binding of importin 13 to the NF-YB/NF-YC heterodimer, but not to the monomeric components, fully explains the previous observation.

\subsubsection{Binding sites in the NF-YB/NF-YC dimer for NF-YA and importin 13 overlap}

Since the minimal regions essential for nuclear localization of NF-YB and NF-YC correspond to the histone fold motifs (as shown by the in vivo transfection experiments, see chapter 3.1.1.3) and only the preformed NF-YB/NF-YC dimer was able to bind to importin 13 (see above) it is reasonable to assume that the binding site in the NF-YB/NF-YC complex for importin 13 consists of the dimerized histone fold motifs. To further prove this assumption, binding of recombinant importin 13 to an immobilized zz-NF-YB/GST-NF-YC complex was performed in the presence of an excess of NF-YA. Similar to importin 13, NF-YA is also not able to interact with either NF-YB or NF-YC alone (Kim et al., 1996b; Sinha et al., 1996; Sinha et al., 1995) but associates specifically with the preformed NF-YB/NF-YC dimer. Furthermore, NF-YA presumably binds to a groove formed by the dimerized histone fold motifs (Romier et al., 2003). Hence, if the binding site in the NF-YB/NF-YC dimer for importin 13 also consists of the dimerized histone fold motifs, binding of importin 13 should be reduced in the presence of NF-YA.

The results of this competition approach indeed showed that importin 13 interacts with the NF-YB/NF-YC complex in a NF-YA sensitive manner. Importin 13 was bound specifically to the histone fold dimer and this binding was abolished by an excess of NF-YA (Fig.13A, note the binding of NF-YA instead of importin 13). To test the specificity of the competition, the GST-NF-YB/GST-NF-YC complex on beads was exposed to purified recombinant importin 13 and increasing amounts of NF-YA. Again, the results show that importin 13 competes with 
NF-YA for binding to the NF-YB/NF-YC dimer (Fig.13B). Addition of equimolar concentrations of importin 13 and NF-YA to the NF-YB/NF-YC complex results in weaker binding of importin 13 (Fig.8B, lane 7) indicating a higher affinity of NF-YA than importin 13 for the NF-YB/NF-YC dimer.
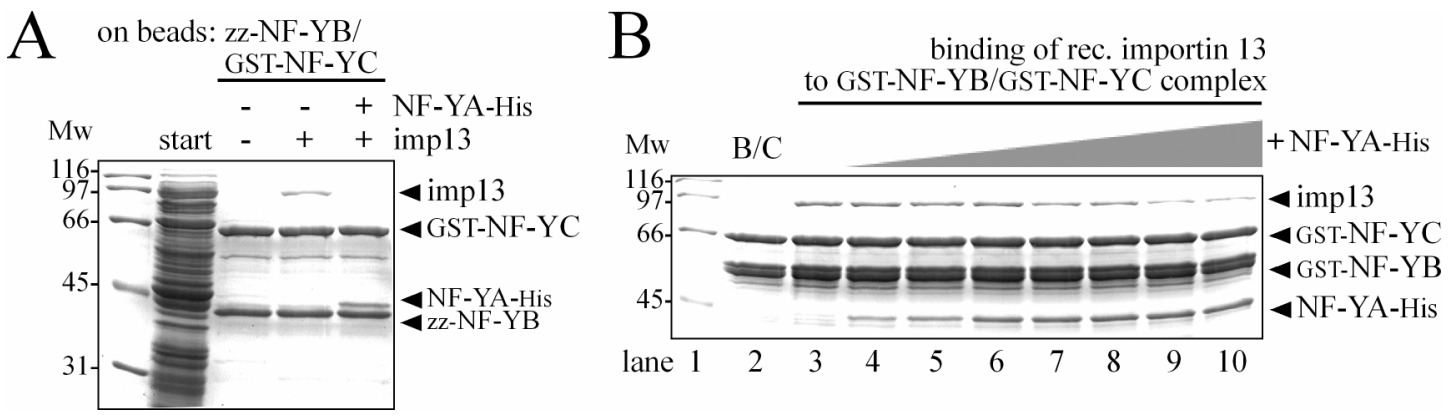

FIG. 13. Binding sites in the NF-YB/NF-YC complex for NF-YA and importin 13 overlap. Importin 13 interacts with the NF-YB/NF-YC complex in a NF-YA sensitive manner. (A) Importin 13 was bound specifically to the immobilized zz-NF-YB/GST-NF-YC complex and this binding was abolished by a 10-fold molar excess of NF-YA. As can be seen in the last lane, NF-YA was bound to the histone fold dimer instead of importin 13. (B) GST-NF-YB/ GST-NF-YC complex on beads (also labeled B/C) was exposed to purified recombinant importin $13(1 \mu \mathrm{M}$, lane 3 to 10$)$ and increasing amounts of His-tagged NF-YA (lane 4: $0.2 \mu \mathrm{M}$, lane 5: $0.4 \mu \mathrm{M}$, lane 6: $0.8 \mu \mathrm{M}$, lane 7: $1 \mu \mathrm{M}$, lane 8: $1.25 \mu \mathrm{M}$, lane 9: $2.5 \mu \mathrm{M}$, lane 10: $5 \mu \mathrm{M}$ ). Again, the results show that importin 13 competes with NF-YA for binding to the NF-YB/NF-YC dimer. Addition of equimolar concentrations of importin 13 and NF-YA to the NF-YB/NF-YC complex (lane 7) results in weaker binding of importin 13 indicating a higher affinity of NF-YA than importin 13 for the NF-YB/NF-YC dimer.

In conclusion, binding sites in the NF-YB/NF-YC dimer for NF-YA and importin 13 overlap. Our data further suggest that the binding site consists of the dimerized histone fold motifs for which NF-YA has the higher affinity.

\subsubsection{Importin $\beta$ binding is restricted to the monomeric, uncomplexed NF-YA subunit}

Since the identified ncNLS of NF-YA also contains the known subunit interaction domain we wanted to find out whether NF-YA can still bind import receptors once it is assembled into the NF-Y complex. To address this question, two kinds of pulldown experiments were performed. First, a GST-NF-YB/GST-NF-YC complex was used as bait and was exposed to a 10-fold excess of both, recombinant importin $\beta$ and/or NF-YA (Fig.14A). NF-YA and importin $\beta$ were added either at the same time (Fig.14A, lane 7) or stepwise, NF-YA first and importin $\beta$ one hour later (Fig.14A, lane 8). Again, bound fractions were analyzed by SDS-PAGE followed by Coomassie staining. When NF-YA was pre-bound to the NF-YB/NF-YC complex no binding of importin $\beta$ 
could be observed, showing that NF-YA was unable to mediate the binding of the entire heterotrimeric NF-Y complex to importin $\beta$. In the presence of an importin $\beta$ excess NF-YA showed reduced binding to the histone fold dimer (data not shown) also indicating that the importin $\beta / \mathrm{NF}-\mathrm{YA}$ complex can not bind to the NF-YB/NF-YC dimer.
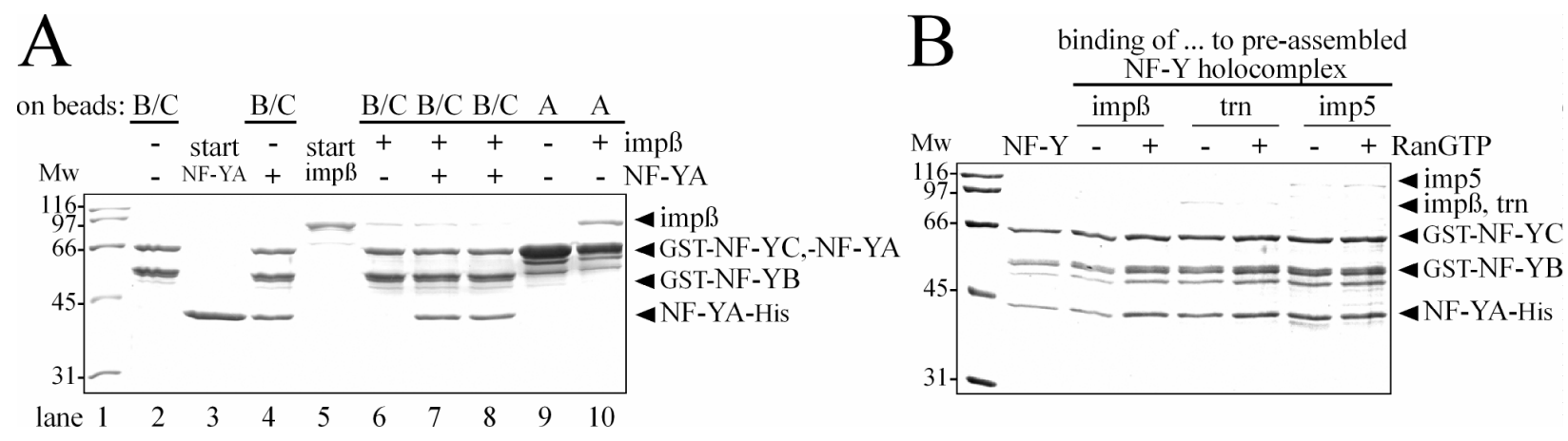

FIG. 14. NLS in NF-YA can not be further recognized by importins once the subunit is assembled into the NF-Y complex. (A) GST-NF-YB/GST-NF-YC (B/C) on beads was exposed to recombinant importin $\beta$ with and without addition of NF-YA to see whether NF-YA can bridge binding of importin $\beta$ to the trimeric NF-Y complex. NF-YA and importin $\beta$ were added either at the same time (lane 7) or stepwise, NF-YA first and importin $\beta$ one hour later (lane 8). Last lane shows the control binding of importin $\beta$ to GST-NF-YA (labeled A) alone. (B) Pre-assembled NF-Y holocomplex (see materials and methods, chapter 2.2.3.3.5.1) consisting of NF-YA-His, GST-NF-YB, and GST-NF-YC was immobilized on glutathione-sepharose beads and incubated with recombinant importin $\beta$, transportin and importin 5. None of the importins that bound to NF-YA alone (see FIG. 12B) were bound to the pre-assembled NF-Y holocomplex. Starting material (20\% of the recombinant importin $\beta$ and NF-YA that were used; panel A) and bound fractions were analyzed by SDS-PAGE followed by Coomassie staining.

Secondly, a pre-assembled NF-Y holocomplex (for preparation, see chapter 2.2.3.3.5.1) was immobilized on glutathione-Sepharose beads and incubated with recombinant importin $\beta$, transportin, and importin 5 (Fig.14B). None of the import factors that specifically bound to the monomeric NF-YA subunit (Fig.12A) showed efficient binding to the pre-assembled NF-Y holocomplex.

In conclusion, the NLS of NF-YA can not be recognized by import factors once the trimeric NF-Y complex is formed which is most likely due to the fact that subunit interaction domain and NLS overlap. Actually the entire subunit interaction domain is part of the mapped NLS, and basic amino acid residues within the subunit interaction domain were shown to be crucial for the nuclear localization of the single NF-YA subunit (see Fig.8; chapter 3.1.1.4). Obviously, these 
amino acid residues can not fulfill their dual function (subunit interaction and nuclear targeting) at the same time.

\subsubsection{NF-Y subunits are imported into nuclei of permeabilized cells by distinct pathways}

Regarding the nuclear transport of the NF-Y subunits, we finally wanted to examine whether the differentially interacting importins represent functional import receptors for NF-YA and the NF-YB/NF-YC dimer. For that purpose, the in vitro nuclear import assay was applied again. As described above, HeLa cells were grown on glass coverslips and the plasma membranes were selectively permeabilized with digitonin to reconstitute nuclear import (Adam et al., 1990). The permeabilized cells were incubated with non-fluorescent GST-tagged NF-Y subunits (respectively control cargoes) and recombinant import factors along with an energy-regenerating system and a Ran mix (Ran) consisting of Ran(GDP) and NTF2 (see also chapter 2.2.6). Import reactions of the non-fluorescent NF-Y subunits were visualized by indirect fluorescence microscopy (see also chapter 2.2.7). The activity/functionality of recombinant import factors was controlled with diverse reference cargoes (Fig.15 panels C, D, and E).

Using this in vitro system before, we observed that the import of fluorescently labeled 6z-NF-YA in the presence of rabbit reticulocyte lysate as source of import factors was abolished by addition of non-fluorescent core histones (as competitor) to the import mix (Fig.10B, chapter 3.1.2.1). These results indicated that the nuclear transport of NF-YA depends entirely on (at least one of) the import receptors that mediate the nuclear transport of the core histones, namely importin $\beta$, importin 5, importin 7, importin 9 and transportin (Baake et al., 2001a; Muhlhausser et al., 2001). Despite the fact that four of the import factors (importin $\beta$, importin 5, importin 7 , and transportin) bound specifically to GST-NF-YA (Fig.12A, chapter 3.1.2.3), GST-NF-YA was only imported into the nuclei of permeabilized cells when importin $\beta$ together with Ran and an energy-regenerating system was present (Fig.15A). Nevertheless, we do not exclude that the other three import factors also mediate nuclear transport of NF-YA in vivo which perhaps could not be reconstituted because of the lower affinity towards the cargo and the experimental conditions chosen.

While the monomeric HFM containing subunits NF-YB and NF-YC (as 6z-fusion proteins) were not imported into nuclei of permeabilized cells (Fig.10C), nuclear accumulation of the GST-NF-YB/GST-NF-YC complex was strictly importin 13-dependent and was stimulated by Ran and an energy regenerating system (Fig.15B). Other importins, such as importin $\beta$ had no significant effect as anticipated from the binding studies. Addition of a NF-YA excess to the import mix led to a reduced nuclear import of the pre-formed NF-YB/NF-YC complex in the 


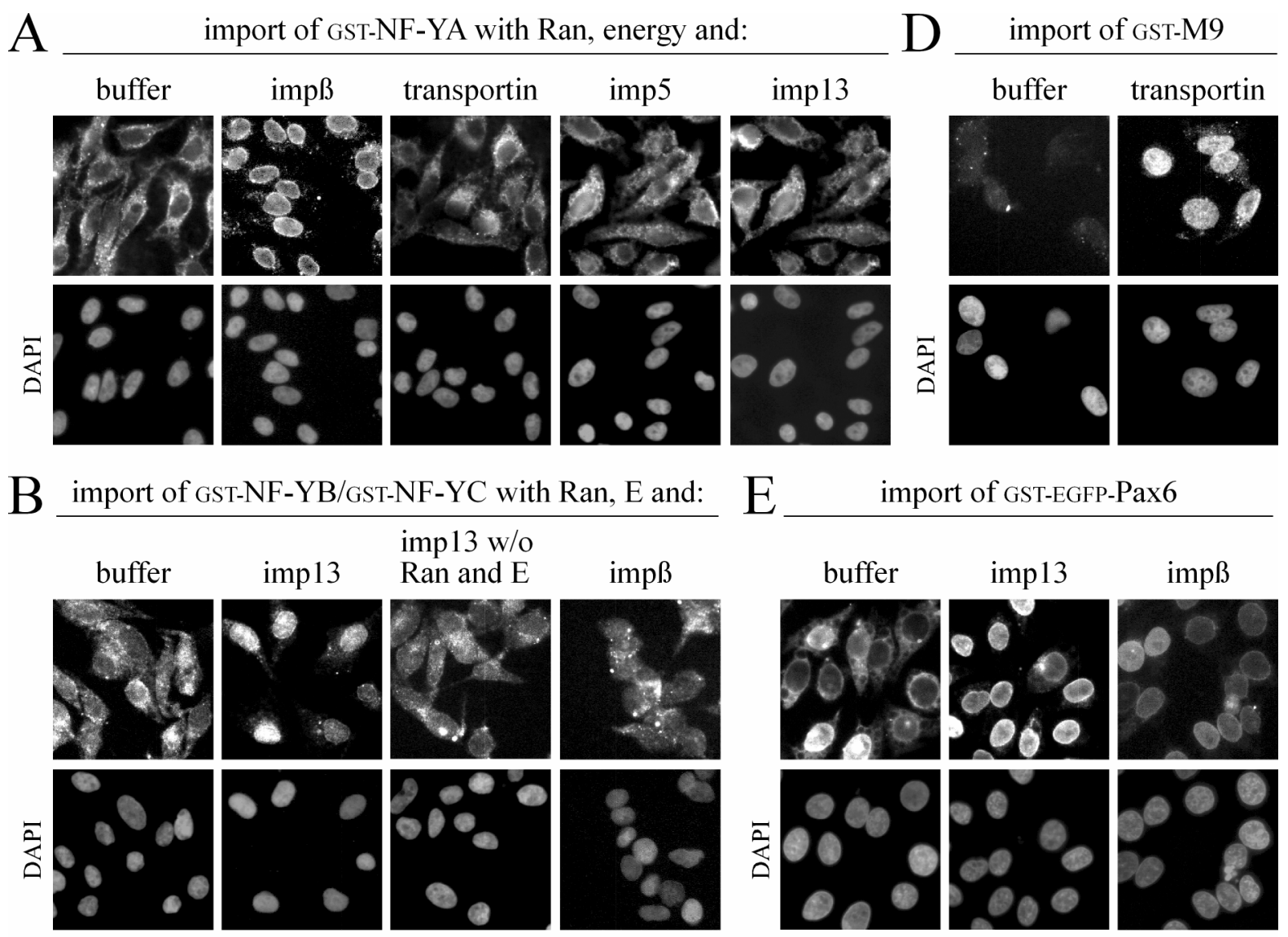

C import of fluorescent 4z-rpL23a with Ran, E and:

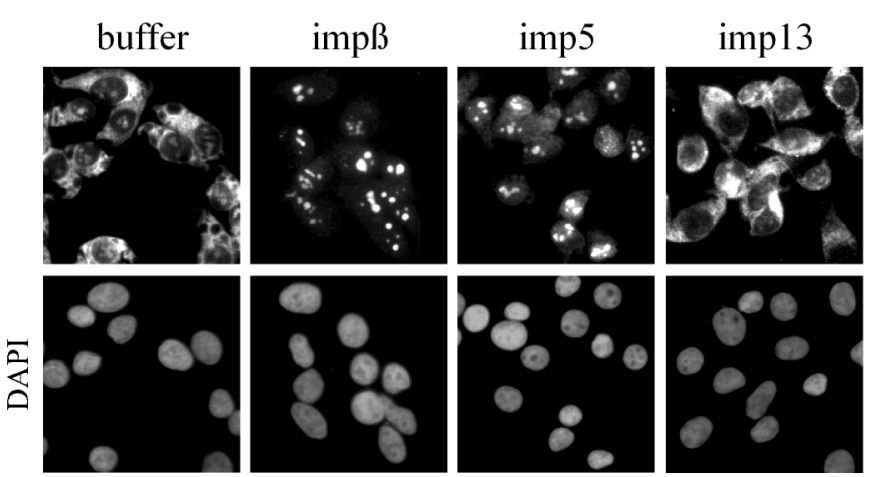

FIG. 15. NF-Y subunits are imported into nuclei of permeabilized cells by distinct pathways. Importin $\beta$ mediates import of NF-YA, and importin 13 confers nuclear uptake of a pre-formed NF-YB/NF-YC dimer. Digitonin-permeabilized cells were incubated with $1 \mu \mathrm{M}$ of each substrate, the indicated combinations of nuclear transport receptors ( $2 \mu \mathrm{M}$ each), a Ran mix (including RanGDP and NTF2) and an energy regenerating system for $25 \mathrm{~min}$ at $37^{\circ} \mathrm{C}$. For a negative control the recombinant import receptor was replaced by transport buffer (buffer). Panels show the cargo distribution after import and fixation. Substrates were detected by indirect immunofluorescence except for the fluorescently labeled rpL23a and GST-EGFP-Pax6. (A) Nuclear accumulation of NF-YA was dependent on the presence of importin $\beta$, Ran mix and an energy-regenerating system. (B) The GST-NF-YB/GST-NF-YC complex was imported into nuclei of permeabilized cells in the presence of importin 13, Ran mix, and an energy regenerating system, but was not imported in the absence of importin 13 or in the absence of Ran and the energy regenerating system. (C, D and E) In vitro import assays of the reference substrates 
4z-rpL23a, GST-M9, and GST-EGFP-Pax6 in the presence and absence of the appropriate nuclear transport receptor.

presence of importin 13 (data not shown).This reduced nuclear import further indicates that only the histone fold dimer can be recognized by importin 13, and that NF-YA competes with importin 13 for binding to the NF-YB/NF-YC dimer (Fig.13). It should be mentioned that neither the HFM containing subunits NF-YB and NF-YC alone nor in combination with each other were tested with transportin in an in vitro import assay. Despite the weak binding of transportin to immobilized GST-NF-YB (Fig.11) no nuclear accumulation of fluorescently labeled 6z-NF-YB was observed in in vitro import assays using rabbit reticulocyte lysate (Fig.10C). Hence, there was no obvious reason to test transportin. The same applies to the NF-YB/NF-YC dimer which apparently could also be not recognized by transportin in vitro (Fig.11).

Taken together, we can conclude that importin $\beta$ functions as import receptor for NF-YA, whereas nuclear transport of the NF-YB/NF-YC dimer indeed can be mediated by importin 13 . Hence, the subunits of the heterotrimeric transcription factor NF-Y are imported into the nucleus by distinct pathways.

\subsection{Nuclear transport of core histones}

Although histones are potentially able to diffuse into nuclei, it was shown that histone H1 is translocated by a receptor-mediated process that excludes diffusion through the nuclear pore complex (Breeuwer and Goldfarb, 1990; Kurz et al., 1997; Schwamborn et al., 1998). In addition, core histones were also found to be actively imported into the nucleus by a receptormediated, energy-dependent pathway. In vitro binding and reconstitution experiments with recombinant import factors indicated that multiple pathways contribute to the nuclear import of the four core histones. In mammalian cells, at least five members of the importin $\beta$ superfamily (namely importin $\beta$, importin 5, importin 7, importin 9, and transportin) mediate the nuclear uptake of monomeric core histones (Baake et al., 2001a; Muhlhausser et al., 2001). While the question of whether the formation of histone heterodimers takes place in the cytoplasm or in the nucleus still has not been completely answered, these previous studies clearly showed that single core histones can be imported into the nucleus of permeabilized cells in vitro. Furthermore, Muhlhausser et al. had reported previously (cited as unpublished data in the publication) that the binding of import receptors to $\mathrm{H} 2 \mathrm{~A}$ and $\mathrm{H} 2 \mathrm{~B}$ monomers was as efficient as the binding to the H2A/H2B heterodimer (Muhlhausser et al., 2001). Since the HFM containing subunits NF-YB 
and NF-YC belong to the $\mathrm{H} 2 \mathrm{~A}-\mathrm{H} 2 \mathrm{~B}$ subfamily (sharing low amino acid sequence identity but high structural resemblance regarding the HFM) the initial question concerned whether the H2A-H2B dimer can also be recognized by importin 13 .

\subsubsection{Importin 13 does not bind to the $\mathrm{H} 2 \mathrm{~A} / \mathrm{H} 2 \mathrm{~B}$ heterodimer}

As mentioned above, the histone fold pair of NF-Y (NF-YB-NF-YC) is closely related to other members of the histone fold family. After the two subunits of the TBP-binding repressor NC2 (NC2 $\alpha-\mathrm{NC} 2 \beta), \mathrm{H} 2 \mathrm{~A}$ and $\mathrm{H} 2 \mathrm{~B}$ represent the second closest relatives and sequence comparison suggests that NF-YB belongs to H2B and NF-YC to the H2A families (Baxevanis et al., 1995). In terms of identity, NF-YB is $30 \%$ identical to $\mathrm{H} 2 \mathrm{~B}$ and NF-YC is $21 \%$ to H2A. As the HFM containing subunits of NF-Y, H2A and H2B dimerize tightly head to tail via their HFMs. Based on the previous result that importin 13 bound specifically to the NF-YB/NF-YC dimer, the obvious next question was directed towards whether dimerization of $\mathrm{H} 2 \mathrm{~A}$ and $\mathrm{H} 2 \mathrm{~B}$ (dimerization of histone fold partners in general) creates a binding interface for importin 13 association.

Thus, a GST-H2A/GST-H2B complex immobilized on glutathione-Sepharose beads was exposed to recombinant importin $\beta$, transportin, and importin 13, all from bacterial lysates. Importin $\beta$ and transportin, but not importin 13 were bound specifically to the immobilized $\mathrm{H} 2 \mathrm{~A}-\mathrm{H} 2 \mathrm{~B}$ complex and this binding was strongly reduced in the presence of RanGTP, which was used again to simulate nuclear Ran conditions (Fig.16A). In contrast to the report of Muhlhausser et al. (Muhlhausser et al., 2001) binding of importin $\beta$ and transportin to the $\mathrm{H} 2 \mathrm{~A} / \mathrm{H} 2 \mathrm{~B}$ dimer was less efficient compared to the H2A and H2B monomers (data not shown). This suggests that the histone import signals (amino terminal tails) were only partially accessible to importin binding when $\mathrm{H} 2 \mathrm{~A}$ and $\mathrm{H} 2 \mathrm{~B}$ were dimerized. However, the pre-assembled H2A-H2B complex consisted of subunits carrying N-terminal GST-tags that might have interfered with subsequent importin binding. Thus, it can not be excluded that these affinity tags caused the lower efficiency.

To reduce the possible interference of GST tags with importin binding also a GST-H2A/zz-H2B complex (Fig.16B) and vice versa (zz-H2A/GST-H2B, data not shown) was used to bind recombinant importin 13 from a bacterial lysate, besides the GST-H2A/GST-H2B complex. However, in contrast to the closely related NF-YB/NF-YC histone fold dimer (Fig.16B, lane 8 and 9) the H2A/H2B complex (Fig.16B, lane 4 and 5) did not behave as an importin 13-specific import substrate suggesting that importin 13 does not bind to histone fold pairs in general. 

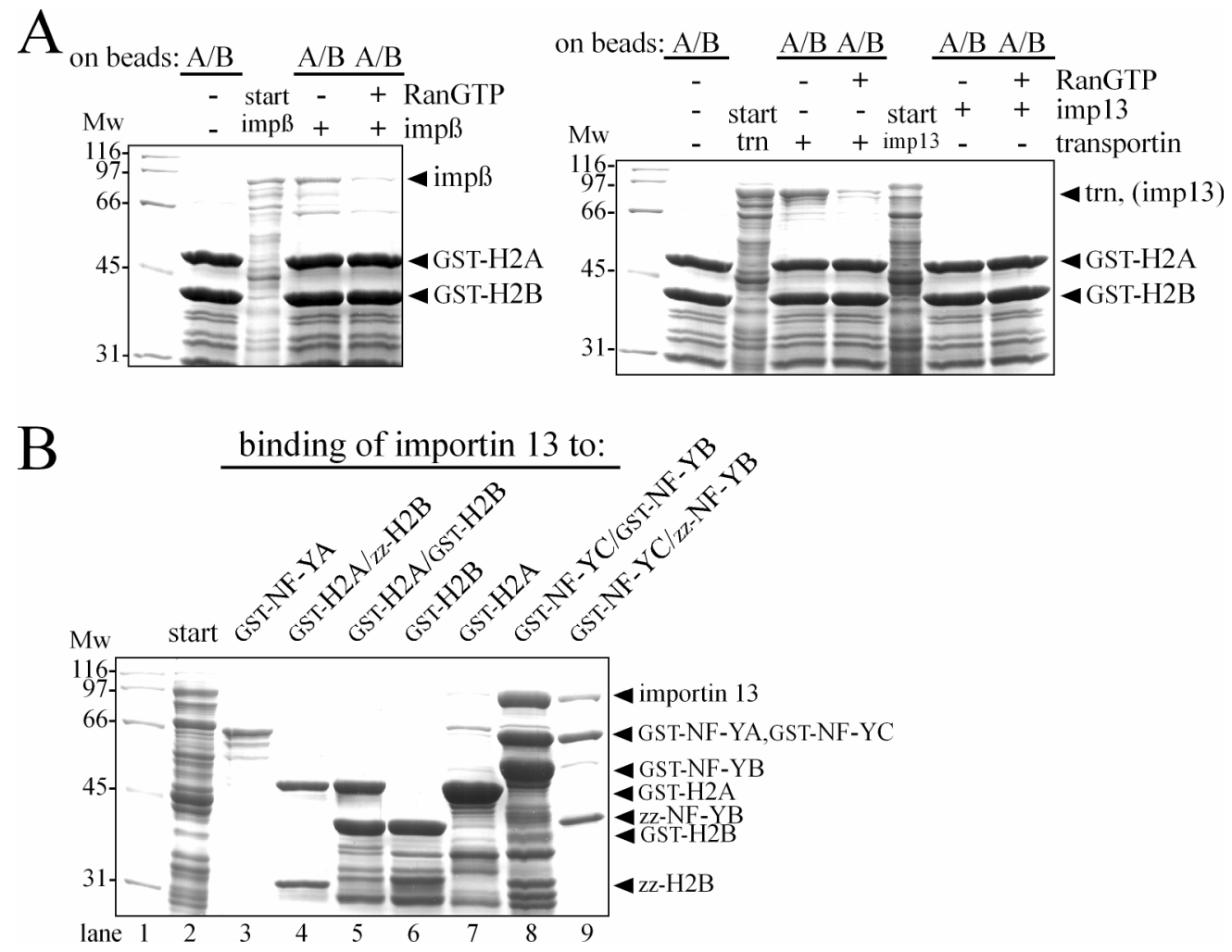

FIG. 16. Importin 13 does not bind to the H2A/H2B heterodimer. GST pull down assays were performed as described in legend to FIG. 11. (A) GST-H2A/GST-H2B (A/B) on beads was exposed to recombinant importin $\beta$, transportin, and importin 13 from bacterial lysates. Importin $\beta$ (left panel) and transportin, but not importin 13 (right panel) were bound specifically to the immobilized H2A-H2B complex and this binding was strongly reduced by RanGTP. (B) Immobilized H2A-H2B and NF-YB-NF-YC (either as zz/GST-fusion or GST/GST-fusion complex; see also arrows on the right) were used to bind recombinant importin 13 from a bacterial lysate. In contrast to the closely related NF-YB/NF-YC histone fold dimer (lane 8 and 9), the H2A/H2B complex (lane 4 and 5) did not behave as an importin 13-specific import substrate.

\subsubsection{Interactions of import receptors with different core histone domains}

As mentioned several times, five members of the importin $\beta$ superfamily (importin $\beta$, importin 5 , importin 7, importin 9, and transportin) were identified to interact directly with single core histones and all of these factors supported core histone import in vitro (Baake et al., 2001a; Muhlhausser et al., 2001). In addition, comprehensive analysis of the nuclear import of the core histones in yeast demonstrated that the import also occurs along redundant pathways in vivo (Mosammaparast et al., 2002b; Mosammaparast et al., 2001). With regard to their structure the four core histones show an overall similarity, consisting of unstructured, basic amino and carboxy-termini and a hydrophobic, central globular domain forming the histone fold motif. Analysis of sequence motifs involved in nuclear targeting of core histones (in vivo transfection 
experiments) demonstrated that both the basic amino-terminal domains and the central globular domains of all core histones can serve as nuclear targeting signals (Baake et al., 2001b).

To investigate whether both domains represent independent NLSs in vitro binding studies were performed (see also chapter 2.2.4.2). The amino-terminus, central globular domain (consisting of the histone fold motif, but also including the C-terminus), and the full length protein of each core histone ( $\mathrm{H} 2 \mathrm{~A}, \mathrm{H} 2 \mathrm{~B}, \mathrm{H} 3$, and $\mathrm{H} 4)$ were expressed as $6 \mathrm{z}$ fusion proteins in E. coli, purified on nickel-NTA-agarose, and then immobilized on IgG-Sepharose beads. The immobilized fusion proteins were incubated with either importin $\beta$, transportin or importin 5, all from bacterial lysates and were subsequently washed. The bound fractions were analyzed by SDS-PAGE followed by Coomassie staining (Fig.17).

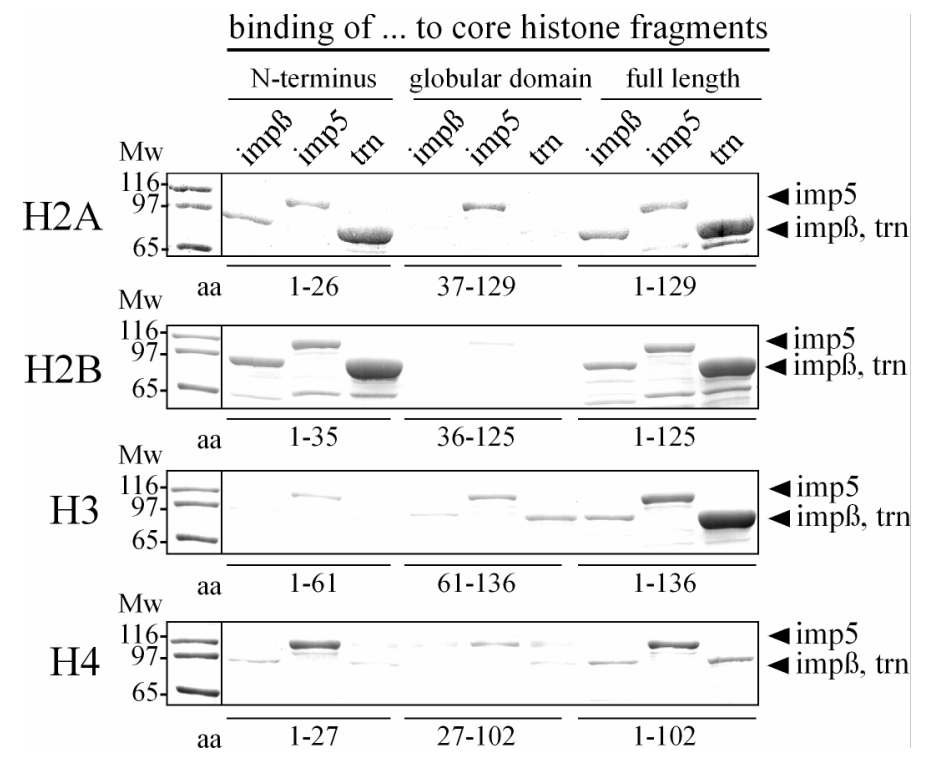

FIG. 17. Interactions of import receptors with different core histone domains. Amino-terminal tail, globular domain (consisting of the histone fold motif) including the C-terminus and full length protein of each of the four core histones (H2A, H2B, H3, and H4) were expressed as $6 \mathrm{z}$ fusions in E. coli, purified on nickel-NTA agarose and subsequently immobilized on IgG-sepharose. The immobilized fusion proteins were incubated with either importin $\beta$, transportin or importin 5 , all from bacterial lysates. The bound fractions were analyzed by SDS-PAGE followed by Coomassie staining. Not surprising, importin $\beta$, transportin, and importin 5 bound to all the full length core histones. Also binding of these transport receptors to the N-terminus of H2A, H2B and H4 (less strong) was observed whereas under the same conditions only weak binding was detectable to the globular domains of the four core histones and the amino-terminal tail of $\mathrm{H} 3$ (in which case much less protein was immobilized compared to the other amino-terminal fusion proteins).

As expected, importin $\beta$, transportin, and importin 5 bound to all of the full length core histones (Fig.17, right side). Also binding of the selected importins to the amino-terminal tails of H2A, 
H2B and H4 (less strong) was observed (Fig.17, left side), whereas under the same conditions only weak binding (mainly of importin 5) was detectable to the globular domains of the core histones (Fig.17, middle sections) and to the amino-terminal tail of H3. It has to be mentioned that especially in the case of the amino-terminal tail of $\mathrm{H} 3$ much less protein was immobilized on the beads compared with the other amino-terminal fusion proteins, which presumably caused the only weak binding. In general, it was rather difficult to immobilize equal amounts of the different core histone domains. These problems occurred because many of the proteins were expressed in inclusion bodies and therefore had to be purified under denaturing conditions (chapter 2.2.3.3.4), more or less successfully. Thus, from the amount of retained import receptors no conclusion can be drawn regarding the binding competence (or strength of affinity).

However, the results of these in vitro binding studies strongly indicated that import factors only interact directly with the amino-terminal tails of core histones, whereas the globular domains were not significantly recognized. This further suggests that the histone fold motif represents rather a histone-histone (protein-protein) interaction domain than an independent NLS. These results are in accordance with the data collected from the two HFM containing subunits of NF-Y. Import factors did also not bind efficiently to the monomeric NF-YB and NF-YC subunits (Fig.11, chapter 3.1.2.2).

\subsubsection{Amino-terminal tails of core histones contain the functional NLSs}

In order to verify that binding of the different members of the importin $\beta$-family to the aminoterminal tails of the core histones is specific, in vitro binding assays were done in the presence and absence of RanGTP which was used to simulate nuclear Ran conditions, as described above. Immobilized zz-H2A, zz-H2B (Fig.18) and zz-H3, zz-H4 (Fig.19) (amino-terminal tails and full length proteins) were used to bind importin $\beta$, transportin, and importin 5 from bacterial lysates. In each case the import receptors were bound specifically to the pre-loaded matrices and this binding was reduced by the GTP-bound form of RanQ69L. Further, the specific binding of the import factors to the amino-terminal tails of $\mathrm{H} 2 \mathrm{~A}, \mathrm{H} 2 \mathrm{~B}, \mathrm{H} 3$, and $\mathrm{H} 4$ was as strong as the binding of importins to the entire (full length) core histones. These results strongly suggest that core histones contain a non-classical NLS (ncNLS) in the amino-terminal tails which is also in accordance with the observations made in lower eukaryotes. In yeast, the amino-terminal tails were previously found to be essential and sufficient for nuclear accumulation of the core histones (Mosammaparast et al., 2002b; Mosammaparast et al., 2001). 
A binding of ... to $\mathrm{N}$-terminus of $\mathrm{H} 2 \mathrm{~A}$

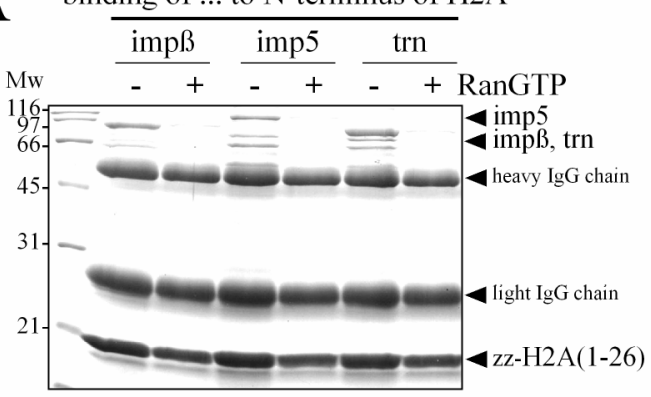

$\mathrm{C}$

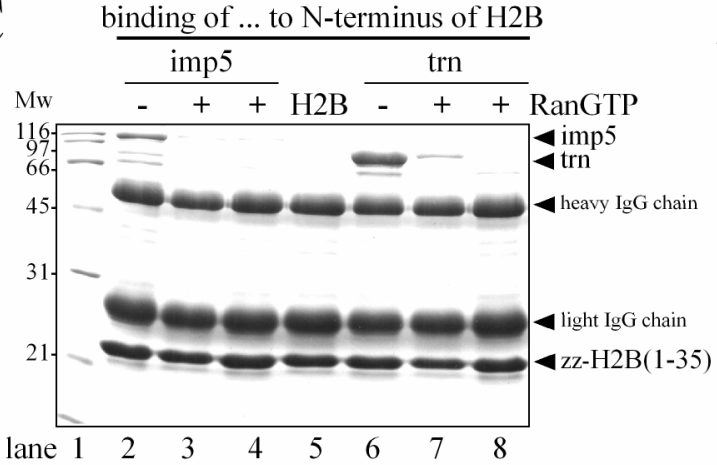

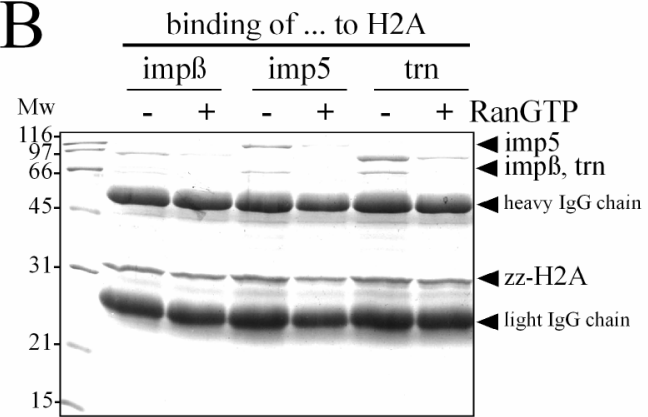

$\mathrm{D}$

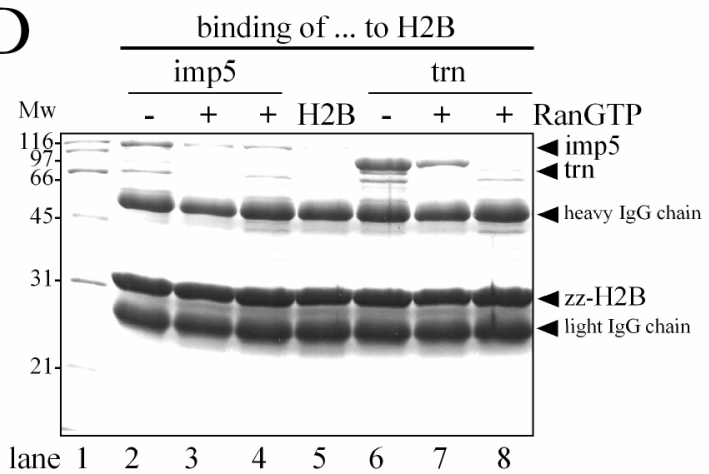

E

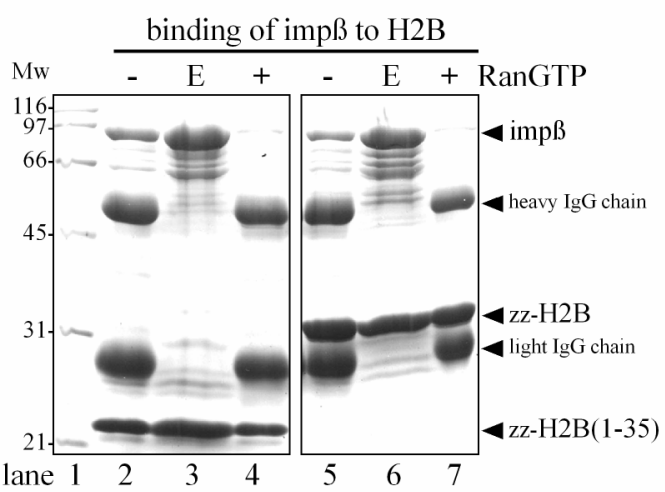

FIG. 18. Importins bind specifically to the amino-terminal tails of $\mathrm{H} 2 \mathrm{~A}$ and H2B. In vitro binding studies were performed essentially as described in the legend to FIG. 17. Immobilized zz-H2A (A, B) and zz-H2B $(C, D, E)$ (either the amino-terminus or the full length protein) were incubated with importin $\beta$, transportin and importin 5, all from bacterial lysates. In each case the import receptors were bound specifically to the pre-loaded matrix and this binding was reduced by the GTP-bound form of RanQ69L $(10 \mu \mathrm{M})$ which was used again to simulate nuclear Ran conditions. To analyze the specificity of the binding, twice the amount of RanGTP $(20 \mu \mathrm{M})$ was added in case of $\mathrm{H} 2 \mathrm{~B}$ (lane 4, 8 in panel $\mathrm{C}$ and $\mathrm{D}$ ). Note, the binding of the importins to the amino-terminal domains of $\mathrm{H} 2 \mathrm{~A}$ and $\mathrm{H} 2 \mathrm{~B}$ was as strong as the binding to the full length core histones indicating that in both histones the amino-terminal tails contain the functional NLS. (E) Matrix-bound material was eluted with $1.5 \mathrm{M}$ $\mathrm{MgCl}_{2}$, was precipitated with isopropanol, and was subsequently analyzed by SDS-PAGE followed by Coomassie staining $(\mathrm{E}=$ eluate $)$. 

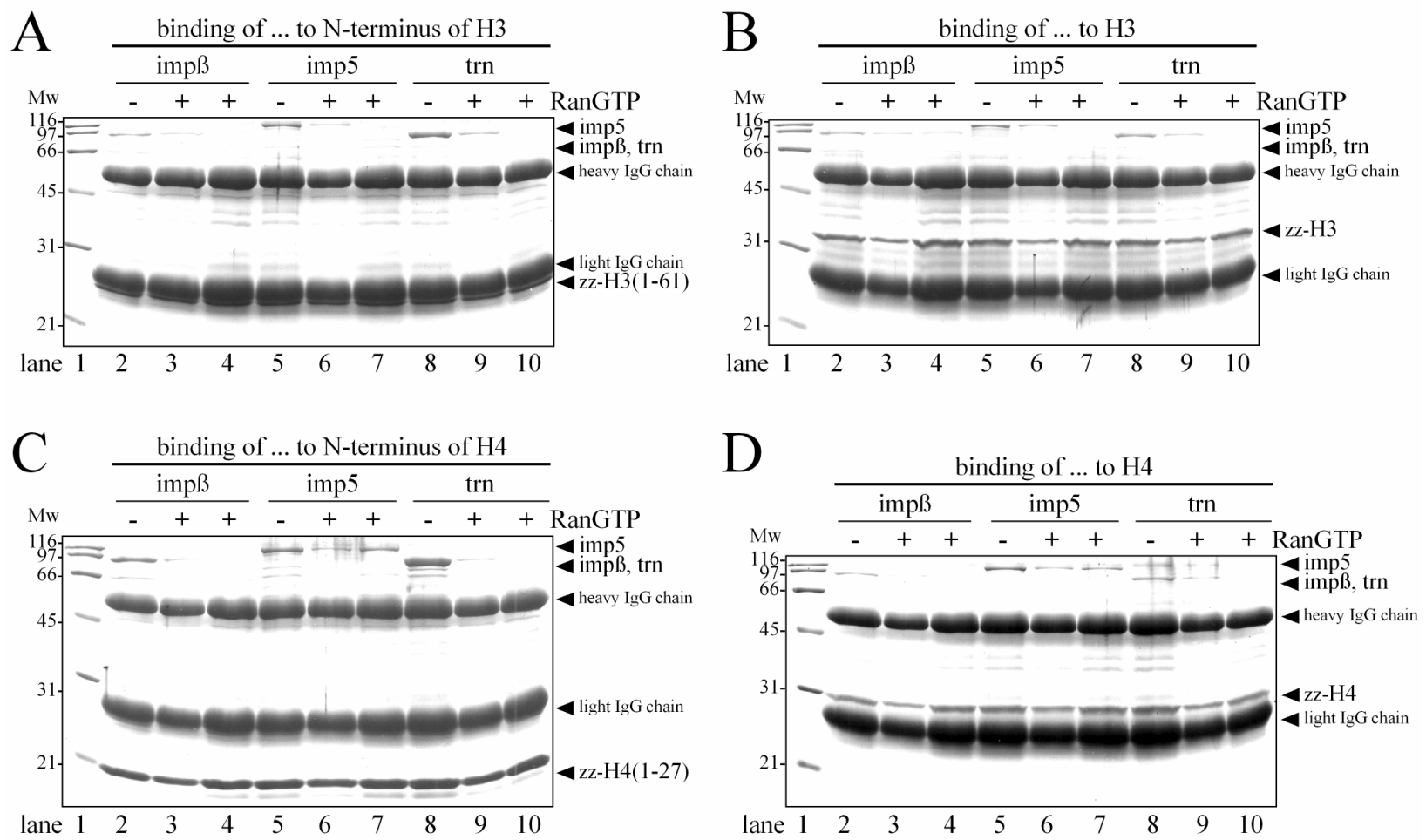

FIG. 19. Importins bind specifically to the amino-terminal tails of $\mathbf{H 3}$ and H4. As in FIG. 18 , immobilized zz-H3 (A, B) and zz-H4 (C, D) (amino-terminal domains and full length proteins) were used to bind importin $\beta$, transportin and importin 5 from bacterial lysates. Binding of these importins to $\mathrm{H} 3$ and $\mathrm{H} 4$ occurred in a RanGTP-sensitive manner, and as for $\mathrm{H} 2 \mathrm{~A}$ and $\mathrm{H} 2 \mathrm{~B}$, both core histones contain a non-classical NLS in the amino-terminal tails (twice the amount of RanGTP was used again in the lanes 4, 7, and 10).

\subsubsection{Globular histone domains are not sufficient to confer nuclear import}

Although the foregoing binding studies had already strongly suggested that the central globular domains of the core histones do not represent an independent NLS, we wanted to examine whether these single domains can be imported into the nuclei of permeabilized cells. For that reason, digitonin-permeabilized HeLa cells were incubated with either the fluorescently labeled, 6z-tag enlarged amino-terminal tails or the globular domains of the different core histones in the presence and absence of rabbit reticulocyte lysate (as general source of import receptors) and an energy regenerating system (Fig.20).

Thereby, we observed strong passive diffusion of the amino-terminal tails of H2A, H2B and H4 into the nuclei of permeabilized cells in the absence of reticulocyte lysate (Fig.20, top panels). Thus, despite of the size enlargement with six z-tags fused to each amino-terminal tail, we could not further analyze the nuclear transport capacity of these domains. As anticipated from the weak binding of import factors to the central globular domains of the four core histones, these domains 
import of the N-terminal domains and the central globular domains of all four core histones

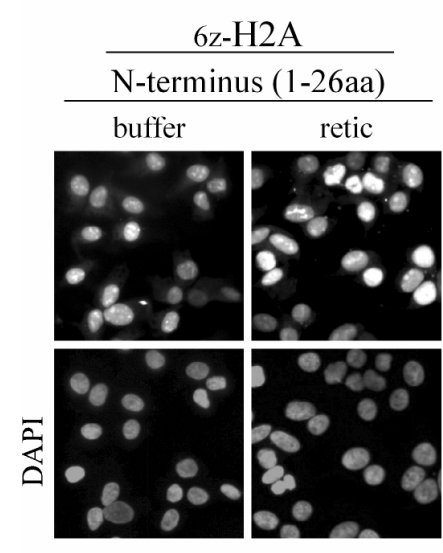

$6 \mathrm{z}-\mathrm{H} 2 \mathrm{~A}$

globular domain (26-129aa)

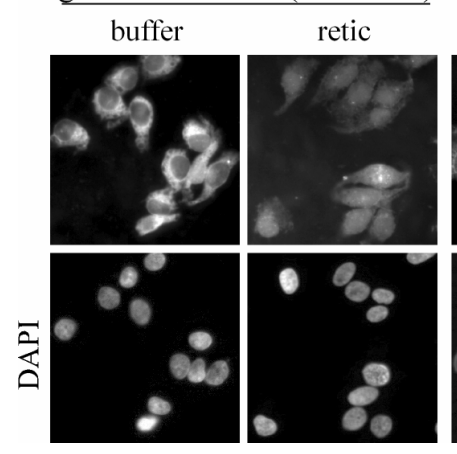

$\frac{\frac{6 z-H 2 B}{\text { N-terminus (1-35aa) }}}{\text { buffer retic }}$
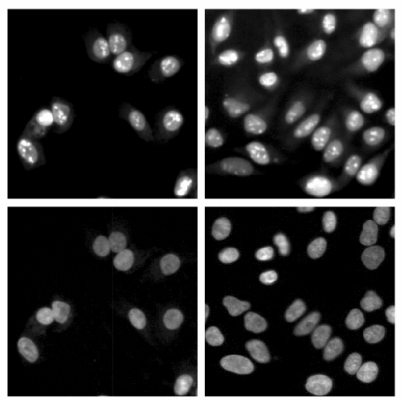

6z-H2B

globular domain (36-125aa)
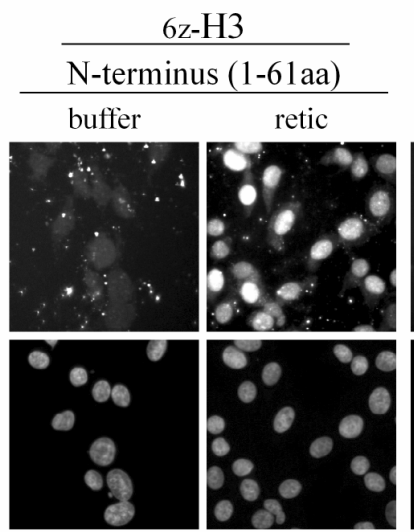

6z-H3

globular domain (61-135aa)

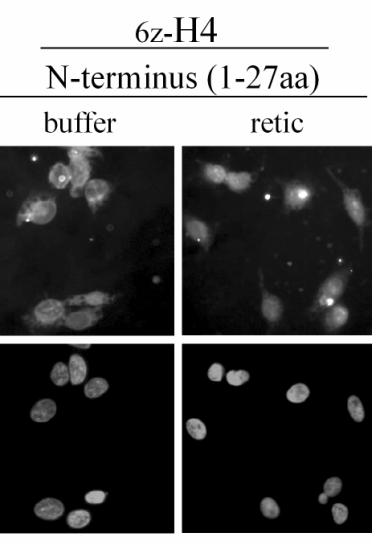

6z-H4
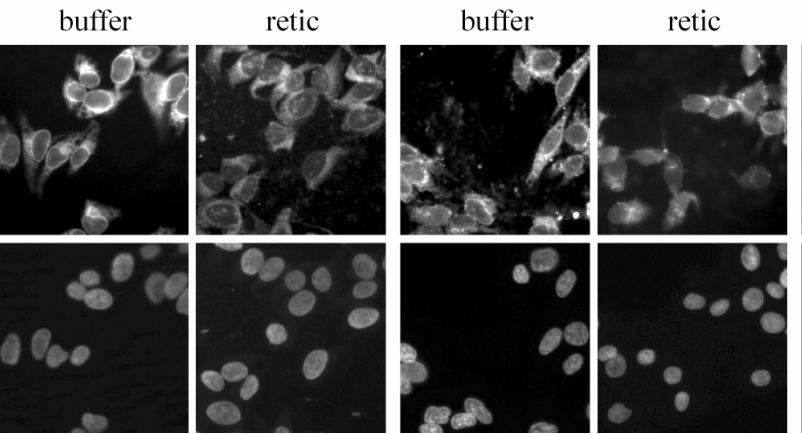

globular domain (27-102aa) buffer
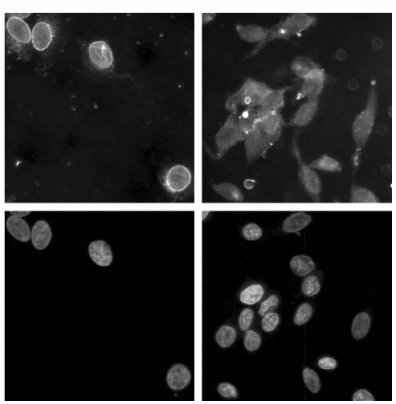

FIG. 20. Globular domains of the core histones $\mathrm{H} 2 \mathrm{~A}, \mathrm{H} 2 \mathrm{~B}, \mathrm{H3}$, and $\mathrm{H} 4$ are not sufficient to confer nuclear accumulation in the nuclei of permeabilized cells. In vitro import assays were performed essentially as described in the legend to FIG. 15. Digitonin-permeabilized HeLa cells were incubated with about $1 \mu \mathrm{M}$ of each fluorescently labeled core histone domain (amino-terminal domain, central globular domain) in the presence (retic) and absence (buffer) of rabbit reticulocyte lysate and an energy regenerating system for 25 min at $37^{\circ} \mathrm{C}$ (for $15 \mathrm{~min}$ at $25^{\circ} \mathrm{C}$ in case of the amino-terminal domains). Reactions were stopped by fixation and the distribution of the fluorescent samples was analyzed with a Zeiss microscope. Note that the amino-terminal tails of $\mathrm{H} 2 \mathrm{~A}, \mathrm{H} 2 \mathrm{~B}$ and $\mathrm{H} 4$ despite of the size enlargement of six z-tags passively diffused into the nuclei of permeabilized cells in the absence of import receptors (buffer).

did also not accumulate in the nuclei of permeabilized cells in the presence of reticulocyte lysate (Fig.20, lower panels). The only notable exception was the globular domain of H2A which at least partially was translocated into the nucleus (Fig.20, lower panel, left side). In that case, however, the globular domain (as tentatively defined) contained parts of the amino-terminal tail (clusters of basic amino acids) that were essential for the nuclear localization of $\mathrm{H} 2 \mathrm{~A}$ in yeast (Mosammaparast et al., 2001). Therefore, it can not be excluded that these basic clusters caused the partial nuclear staining of the globular domain of $\mathrm{H} 2 \mathrm{~A}$. 
Anyway, based on our previous transfection experiments (Baake et al., 2001b) we assumed that the central globular domains of the core histones containing the histone fold motif as main structural feature represent a second NLS in core histones (besides the amino-terminal tails). Since this type of signal would have been defined by its secondary/tertiary structure rather than by its primary amino acid sequence the central globular domains of all four core histones should have been recognized and imported by transport receptors. However, this was neither the case regarding the core histones nor for the histone fold motif containing subunits NF-YB and NF-YC (chapter 3.1.2). Thus, the histone fold motif represents a protein-protein interaction domain but definitely not a NLS per se.

\subsubsection{Nuclear transport competence of the globular domains of core histones}

If central globular domains of core histones do not contain or by themselves represent an independent NLS the nuclear transport competence of these domains (as demonstrated by Baake et al., 2001b in transfection experiments) should entirely depend on the formation of histone dimers (oligomers). To test this theory, in vitro nuclear import assays were performed with the fluorescently labeled globular domain of H2B in the presence and absence of the corresponding full length partner histone H2A (Fig.21). This artificial dimeric complex consisting of the fluorescently labeled, 6z-tagged globular domain of H2B and non-fluorescent GST-H2A was pre-assembled. In detail, both fusion proteins were independently expressed in E. coli, affinity purified, and in the case of H2B fluorescently labeled. GST-H2A was immobilized on glutathione-Sepharose beads and used as bait to bind the fluorescently labeled, 6z-tagged globular domain of H2B. The artificial histone dimer was then eluted with glutathione, dialyzed against transport buffer (supplemented with $300 \mathrm{mM} \mathrm{NaCl}$ to avoid precipitation of the core histones; see also chapter 2.2.3.3.5.2), and finally used as transport cargo in the in vitro nuclear import assay.

In contrast to the single (monomeric) globular domain of H2B (Fig.21, left panels) the preassembled complex consisting of the fluorescently labeled globular domain of $\mathrm{H} 2 \mathrm{~B}$ and full length H2A was imported into the nucleus in the presence of reticulocyte lysate and an energy regenerating system (Fig.21, right panels). Thus, using the in vitro import assay we showed that the globular domain of H2B was piggyback transported into the nucleus via the ncNLS in the amino-terminal tail of the corresponding partner histone H2A. These results further suggest that the NLSs in the amino-terminal tails of core histones are still accessible for import factors even when histones are dimerized (oligomerized). The nuclear transport competence of the globular domains, however, depends entirely on the formation of histone oligomers. 


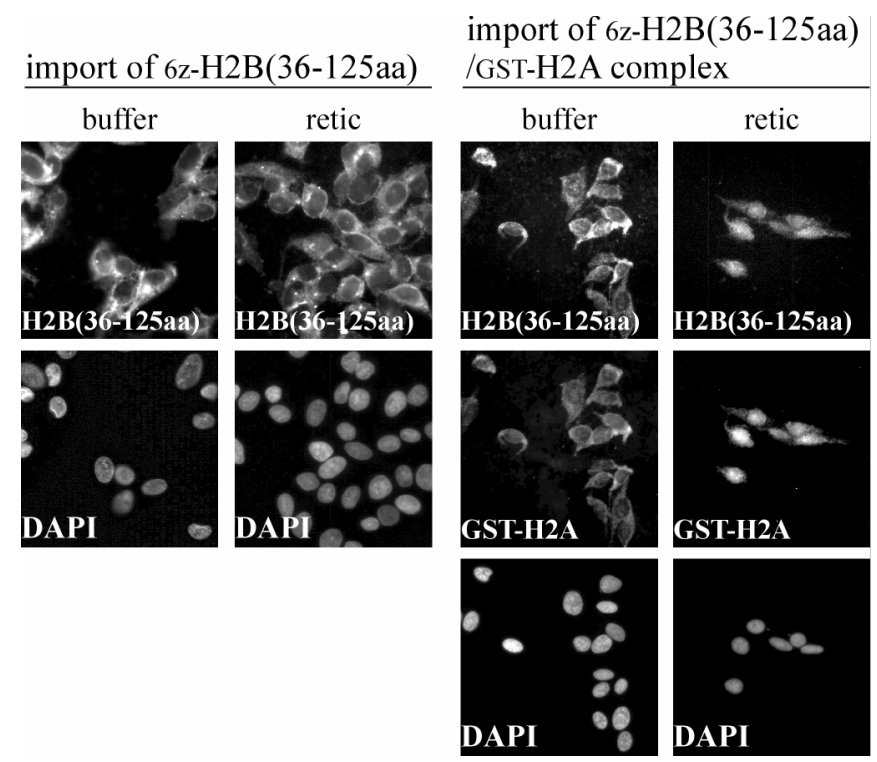

FIG. 21. Piggyback transport of the globular domain of H2B. The nuclear transport competence of globular domains of core histones depends on the formation of histone oligomers. Nuclear import of about $1 \mu \mathrm{M}$ of fluorescently labeled globular domain of $\mathrm{H} 2 \mathrm{~B}$ was performed in the presence (retic) and absence (buffer) of rabbit reticulocyte lysate, an energy regenerating system, and (where indicated) the corresponding full length partner histone $\mathrm{H} 2 \mathrm{~A}$ for $25 \mathrm{~min}$ at $37^{\circ} \mathrm{C}$. In contrast to the globular domain of $\mathrm{H} 2 \mathrm{~B}(6 \mathrm{z}-\mathrm{H} 2 \mathrm{~B}(36-125 \mathrm{aa})$, left panels) the preassembled 6z-H2B(36-125aa)/GST-H2A complex was imported in the nuclei of permeabilized cells in the presence of reticulocyte lysate and an energy regenerating system (right panels). This indicates that the NLS in the aminoterminal tail of H2A was still accessible to importin binding when $\mathrm{H} 2 \mathrm{~A}$ and the globular domain of $\mathrm{H} 2 \mathrm{~B}$ were dimerized.

\subsubsection{Importin $\beta$ uses the same binding site to recognize IBB (BIB) domain and H2B}

After identification of the functional NLS (importin binding site) in core histones, we finally wanted to identify the binding sites in transport receptors for the core histones. To address this question, we focused on mapping the binding site in importin $\beta$ for the core histone H2B. In an oversimplified picture two cargo binding sites exist in importin $\beta$ (see also chapter 1.4). The cargo binding site I (or IBB binding domain) consists of the HEAT repeats 7 to 19 (Cingolani et al., 1999) while the second cargo binding site in importin $\beta$ includes the HEAT repeats 1 to 11 (Cingolani et al., 2002). It should be mentioned that the second cargo binding site was revealed by the crystal structure of an importin $\beta$ fragment bound to the ncNLS of the parathyroid hormone-related protein (PTHrP) (Cingolani et al., 2002). Since core histones and the PTHrP are translocated through the NPC directly bound to importin $\beta$, we wanted to examine whether H2B uses the second cargo binding site in importin $\beta$ as well. 
For that purpose, in vitro binding studies were performed using the BIB (beta-like import receptor binding) domain of rpL23a and the IBB (importin $\beta$ binding) domain of importin $\alpha$ as substrates that bind to the cargo binding site I of importin $\beta$. In detail, GST-H2B immobilized on glutathione-Sepharose beads was exposed to either recombinant importin $\beta$ from a bacterial lysate (lanes 3-5) or purified recombinant importin $\beta$ (lanes 6-8) with and without addition of the BIB or IBB domain (Fig.22).

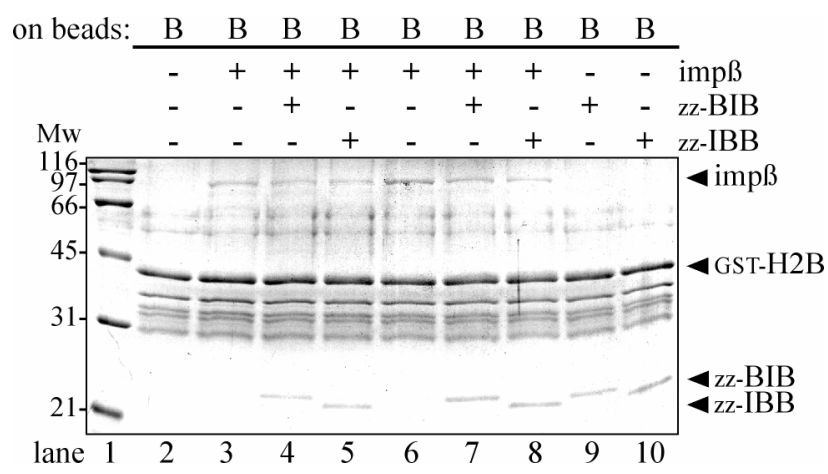

FIG. 22. Core histones presumably bind to the cargo binding site I on importin $\beta$. GST pulldown assays were performed as described in the legend to FIG. 11. GST-H2B (B) on beads was exposed to either recombinant importin $\beta$ from a bacterial lysate (lanes 3-5) or purified recombinant importin $\beta$ (lanes 6-8) with and without addition of zz-BIB (BIB domain of rpL23a) and zz-IBB (IBB domain of importin $\alpha$ ) where indicated. zz-IBB and zz-BIB were used as potential second cargo to investigate whether simultaneous binding of $\mathrm{H} 2 \mathrm{~B}$ and IBB domain (H2B and BIB domain) can occur. However, instead of simultaneous binding the binding of importin $\beta$ to $\mathrm{H} 2 \mathrm{~B}$ was reduced in the presence of zz-IBB and zz-BIB indicating that H2B and IBB (BIB) binding sites in importin $\beta$ overlap. Lanes 9 and 10 show the unspecific binding of zz-BIB and zz-IBB (importin $\beta$ cargoes) to immobilized GST-H2B.

The BIB and IBB domain were used as potential second cargo of importin $\beta$ to investigate whether simultaneous binding of $\mathrm{H} 2 \mathrm{~B}$ and $\mathrm{IBB}$ (H2B and $\mathrm{BIB}$ ) to importin $\beta$ can occur. Cooperative binding would have been a strong hint that these proteins (signals) use different binding sites in importin $\beta$, or rather that $\mathrm{H} 2 \mathrm{~B}$ would have used the second cargo binding site. Unfortunately, because of strong unspecific binding of BIB and IBB domain to immobilized GST-H2B (Fig.22, lane 9 and 10) simultaneous binding could not be investigated unambiguously. However, the binding of importin $\beta$ to GST-H2B was reduced in the presence of these additional cargoes (Fig.22, lane 7 and 8, compared to lane 6) which indicates that H2B and IBB (BIB) binding sites on importin $\beta$ overlap. In accordance to these results, Muhlhausser et al. had reported that the in vitro nuclear uptake of core histones could be completely inhibited by addition of an excess of BIB domain (Muhlhausser et al., 2001). 
Altogether, these results suggest that the ncNLSs (amino-terminal tails) of the core histones most likely bind to the cargo binding site $\mathrm{I}$ in importin $\beta$. Thus, the parathyroid hormone-related protein remains the only substrate which is known to bind to the second cargo binding site on importin $\beta$.

The obtained results regarding the characterization of the mechanisms underlying the nuclear transport of the core histones and the transcriptional activator NF-Y can be briefly summarized as follows.

In vivo transfection experiments demonstrated that both the amino-terminal tails and the central globular domains of all core histones can serve as regions involved in nuclear targeting. However, in vitro binding studies and nuclear import assays revealed that import receptors only interact directly with the amino-terminal tails of core histones. The globular histone domains containing a histone fold motif (HFM) are not recognized by importins which suggests that the HFM represents a protein-protein interaction domain, but not a NLS per se.

Regarding the heterotrimeric transcriptional activator NF-Y, in vitro binding studies and nuclear import assays revealed two different nuclear transport mechanisms for its subunits: (i) NF-YA is imported by an importin $\beta$ mediated pathway, and (ii) the NF-YB/NF-YC heterodimer is translocated into the nucleus in an importin 13 dependent manner. NF-YA contains a nonclassical nuclear localization signal (ncNLS) in the conserved region at the C-terminus of the protein, and mutational analysis indicates that basic amino acid residues in the ncNLS of NF-YA are required for nuclear targeting. Only the NF-YB/NF-YC dimer, but not the monomeric components, are recognized by importin 13, and a distinct binding interface derived from the HFMs of both subunits presumably mediates the interaction with importin 13 . 


\section{Discussion}

A major effort in the field of nucleocytoplasmic transport is the identification of cargoes for the individual importin $\beta$ family members that mediate virtually all of the transport events between nucleus and cytoplasm. Indeed, more than $17 \%$ of all eukaryotic proteins are nuclear (Cokol et al., 2000) and therefore must be transported into the nucleus at some stage of their life. Among the proteins whose role is clearly nuclear are histones accompanied by the numerous factors associated with the chromatin assembly machinery and proteins mediating the gene expression programs such as transcriptional activators and repressors. While nuclear import of (core) histones relies on a network of import receptors which seem to have a certain preference for their cargoes little was known regarding the mechanism by which the ubiquitous CCAAT-specific transcription factor NF-Y is translocated into the nucleus. Thus, the main aim in the present work was to examine and to characterize the nuclear transport pathway of the heterotrimeric transcription factor NF-Y. Since the two NF-Y subunits NF-YB and NF-YC belong to the $\mathrm{H} 2 \mathrm{~A} / \mathrm{H} 2 \mathrm{~B}$ subfamily containing a histone fold motif(HFM) as main structural feature the role of this element for the nuclear import of both, the HFM containing NF-Y subunits and the four different core histones, was the second major goal of this study. The aim to characterize the mechanisms underlying the nuclear transport of NF-Y basically had emerged from the first results regarding the role of the HFM for the nuclear transport of the core histones. Therefore, these data are discussed first.

\subsection{Nuclear transport of core histones: abundant cargoes, redundant pathways}

The group of histones, the major structural proteins of chromatin, comprise the four core histones H2A, H2B, H3, H4 and the H1 linker histones (Allan et al., 1986; Pruss et al., 1996; Wolffe, 1998). Histones are among the most abundant cargoes for nuclear import during the S-phase of the cell cycle because the formation of (new) nucleosomes on replicated DNA requires a huge amount of these proteins. As all proteins encoded in nuclear DNA histones are synthesized in the cytoplasm. Therefore, histones need to cross the nuclear envelope to fulfill their nuclear function and an efficient nuclear transport system must assure an adequate supply of histone proteins during the S-phase. Since histones are smaller than the exclusion size of nuclear pore complexes (NPCs), nuclear transport of histones based on diffusion might in principle be possible. However, diffusion would not suffice to provide (in a regulated way) the S-phase nucleus with the high amount of histones needed during the short period of DNA replication. Hence, it was shown that histones are transported into the nucleus in a signal dependent, energy dependent, receptor-mediated process through the NPC (Breeuwer and 
Goldfarb, 1990; Kurz et al., 1997; Schwamborn et al., 1998). Further, it was revealed that a number of different transport factors are involved in the nuclear import of histones (Baake et al., 2001a; Bauerle et al., 2002; Greiner et al., 2004; Jakel et al., 1999; Mosammaparast et al., 2002b; Mosammaparast et al., 2001; Muhlhausser et al., 2001). With regard to the core histones redundant transport pathways exist and specific importins serve as primary or predominant receptors, at least in yeast (Greiner et al., 2004; Mosammaparast et al., 2002b; Mosammaparast et al., 2001). The biochemical mechanisms that determine the histone preference of importins remain unclear; however, intrinsic differences in structure and the involvement of other factors might play a role in this specificity. In mammalian cells, at least five members of the importin $\beta$ superfamily (importin $\beta$, importin 5 , importin 7 , importin 9, and transportin) mediate the nuclear accumulation of core histones (Baake et al., 2001a; Muhlhausser et al., 2001). In yeast, histones H2A and H2B utilize Kap114p as their primary transport receptor (Mosammaparast et al., 2001), while either Kap123p according to (Mosammaparast et al., 2002b) or Kap121p according to (Greiner et al., 2004) fulfills this function for H3 and H4. Because neither Kap114p nor Kap123p are essential, additional pathways of transport must exist for the import of core histones. In line with that, Kap121p, Kap123p, Kap95p, Kap104p were found to serve as secondary receptors for H2A/H2B, whereas Kap119p and Kap108p most likely back up nuclear import of H3 and H4 (Greiner et al., 2004; Mosammaparast et al., 2002b; Mosammaparast et al., 2001). The somewhat divergent data about the requirements for individual importins is probably due to the fact that in these studies (mentioned above) different GFP reporter proteins were used. The question whether also in higher eukaryotes import receptors have a certain preference for the different core histones (as observed in yeast) was not addressed in this study. The reason for this difficulty is on one hand that the purified recombinant transport factors are not equally active. Hence, it is almost impossible to identify primary or predominant receptors in vitro (nuclear import assays) because the importins do not allow detailed comparison regarding their ability to translocate a given cargo. On the other hand, it was also difficult to immobilize equal amounts of the different core histone (domains) for in vitro binding studies. These problems occurred because many of the proteins were expressed in inclusion bodies and therefore had to be purified under denaturing conditions, more or less successfully. Thus, from the amount of retained import receptors also no conclusion could be drawn regarding the binding competence of the import receptors to the immobilized histones.

In addition to the import receptors (mentioned above) which are capable to mediate nuclear transport of monomeric core histones, we also asked whether there are import receptors that 
recognize only dimerized (oligomerized) histones. This question arose on the basis of our results regarding the nuclear transport of the two HFM containing subunits NF-YB and NF-YC (see chapter 4.2.1). The results clearly showed that only the NF-YB/NF-YC dimer, but not the monomeric components, are recognized by importin 13. Since NF-YB and NF-YC belong to the $\mathrm{H} 2 \mathrm{~A}$ and $\mathrm{H} 2 \mathrm{~B}$ families (NF-YB is $30 \%$ identical to $\mathrm{H} 2 \mathrm{~B}$ and $\mathrm{NF}-\mathrm{YC}$ is $21 \%$ identical to H2A) (Baxevanis et al., 1995) we asked whether the H2A-H2B dimer can also be recognized by importin 13. Like the two HFM containing subunits NF-YB and NF-YC, H2A and H2B dimerize tightly head to tail via their HFMs. However, in contrast to the closely related NF-YB/NF-YC dimer the $\mathrm{H} 2 \mathrm{~A} / \mathrm{H} 2 \mathrm{~B}$ complex did not behave as an importin 13-specific import substrate suggesting that importin 13 presumably does not bind to histone fold pairs in general (Fig.16).

\subsubsection{Globular histone domains versus amino-terminal tails}

The structure of the four core histones shows an overall similarity, consisting of unstructured, basic amino-terminal and carboxy-terminal domains and a hydrophobic, central globular domain that forms the histone fold motif (HFM) (Arents and Moudrianakis, 1995; Baxevanis et al., 1995; Luger et al., 1997). The HFM is necessary to form H2A-H2B dimers or (H3-H4) 2 tetramers (Freeman et al., 1996; Luger et al., 1997). Previous studies (in vivo transfection experiments) in our group had revealed that both the basic amino-terminal tails and the central globular domains of all core histones (fused to $\beta$-galactosidase as reporter protein) serve as regions involved in nuclear targeting (Baake et al., 2001b). To extend these studies and to elucidate whether both regions contain functional import signals (NLSs) we performed in vitro binding studies and in vitro import assays (reconstitution experiments). The results of these in vitro studies demonstrated that import factors only interact directly with the amino-terminal tails of core histones, whereas the globular domains were neither significantly recognized by importins nor translocated in the nucleus in the presence of reticulocyte lysate (general source of import receptors) and an energy-regenerating system. These results were in line with the data obtained investigating the nuclear transport of the two HFM containing subunits NF-YB and NF-YC (chapter 4.2.1) and strongly suggest that the HFM represents a histone-histone (protein-protein) interaction domain, but not an independent NLS. Thus, the nuclear localization (transport competence) of a globular histone domain in vivo is most likely due to the interaction with the

corresponding endogenous (newly synthesized) partner core histone in the cytoplasm of transfected cells. This assumption was confirmed by in vitro nuclear import assays showing that the fluorescently labeled globular domain of H2B (without amino-terminal tail upon) was imported into the nucleus in the presence of the corresponding full length partner histone $\mathrm{H} 2 \mathrm{~A}$ 
(described and discussed in the chapter below). Further, we proved the specificity of importin binding to the amino-terminal tails of core histones by addition of RanQ69L(GTP). This point mutant of Ran only occurs in the GTP-bound form, as it can not hydrolyze GTP at a significant rate (Marshallsay et al., 1996; Stewart et al., 1998b). The most important diagnostic feature importins have in common is the amino-terminal RanGTP binding motif and binding of RanGTP to the import receptor triggers dissociation of the importin-cargo complex. The strong reduction of importin binding to the pre-loaded matrices in the presence of RanQ69L(GTP) confirmed the specificity of the interactions. Further, (specific) binding of import factors to the amino-terminal tails of $\mathrm{H} 2 \mathrm{~A}, \mathrm{H} 2 \mathrm{~B}, \mathrm{H} 3$, and $\mathrm{H} 4$ was as strong as binding of importins to the full length core histones. Taken together, these results imply that core histones contain a non-classical NLS (ncNLS) in the amino-terminal tails which is in perfect agreement to the observations made in lower eukaryotes (Mosammaparast et al., 2002b; Mosammaparast et al., 2001).

\subsubsection{Piggyback transport of globular histone domains}

If central globular domains of core histones do not contain or by themselves represent an independent NLS (which would have been defined by its secondary/tertiary structure rather than by its primary amino acid sequence) nuclear transport competence of these domains should depend on the formation of histone oligomers. This possibility was supported by the results of Kolodrubetz et al. and Schuster et al. who studied the viability of yeast mutants with deletions in histones H2A and H2B in vivo (Kolodrubetz et al., 1982; Schuster et al., 1986). Briefly, deletions of the amino-terminal tails of either $\mathrm{H} 2 \mathrm{~A}$ or $\mathrm{H} 2 \mathrm{~B}$ did not affect the viability of the yeast cells. Additionally, the respective regions were interchangeable between $\mathrm{H} 2 \mathrm{~A}$ and $\mathrm{H} 2 \mathrm{~B}$ whereas simultaneous deletions of the amino-terminal tails of both core histones were lethal. Based on these results the authors discussed the possibility that the amino-terminal tails might be involved in nuclear targeting of $\mathrm{H} 2 \mathrm{~A}-\mathrm{H} 2 \mathrm{~B}$ dimers. To test this hypothesis, we performed in vitro nuclear import assays with a pre-assembled complex consisting of the fluorescently labeled globular domain of $\mathrm{H} 2 \mathrm{~B}$ and the corresponding full length partner histone $\mathrm{H} 2 \mathrm{~A}$. In contrast to the monomeric globular domain of $\mathrm{H} 2 \mathrm{~B}$ (which was retained in the cytoplasm) the globular domain of $\mathrm{H} 2 \mathrm{~B}$ in the pre-assembled complex was imported into the nucleus in the presence of reticulocyte lysate and an energy-regenerating system (Fig.21). This in vitro experiment clearly illustrated that the globular domain of $\mathrm{H} 2 \mathrm{~B}$ can be piggyback transported into the nucleus via the ncNLS located in the amino-terminal tail of the partner histone H2A. Thus, this observation also confirmed (i) that the nuclear transport competence of globular domains of core histones depends on the formation of histone oligomers and (ii) that the NLSs in the amino-terminal tails of core 
histones are still accessible to importin binding when histones are dimerized (presumably even when oligomerized). In this context, Muhlhausser et al., 2001 had reported previously (cited as unpublished data in the publication) that the binding of import receptors to $\mathrm{H} 2 \mathrm{~A}$ and $\mathrm{H} 2 \mathrm{~B}$ monomers was as efficient as the binding to the H2A/H2B heterodimer (Muhlhausser et al., 2001). Given the fact that the import signals are still accessible, dimerized $\mathrm{H} 2 \mathrm{~A} / \mathrm{H} 2 \mathrm{~B}$ can presumably interact with two (different) transport receptors, and therefore might use either both import factors simultaneously or only one or the other to translocate through the nuclear pore complex into the nucleus. However, nuclear transport of (possibly) dimerized core histones can and should not be compared with the real piggyback transport, often used by transcriptional regulators lacking functional targeting signals (for details see also chapter 1.7.1). In contrast to the nuclear transport of core histones, a piggyback transport mechanism provides the cell with a supplementary regulation level because both the transport signal and the interaction between cargo and targeting signal carrying protein can be modulated.

\subsubsection{Role of histone chaperones in the nuclear transport of histones}

Although the nuclear transport competence of globular domains of core histones most likely depends on formation of histone heterodimers we can not exclude that interactions of the globular domains with other NLS-containing proteins mediate nuclear transport of these domains (and perhaps nuclear transport of core histones in vivo). Based on the fact that a huge amount of core histones during the S-phase of the cell cycle has to be transported, such a potential histonebinding protein would require a high transport capacity and should be able to efficiently shuttle between nucleus and cytoplasm. The study of histone chaperones (factors involved in chromatin assembly) revealed several proteins which bind directly to histones and carry them to chromatin. Among them, the nucleosome assembly factors N1/N2 (Laskey and Dingwall, 1993) and CAF-1 (Verreault et al., 1996) which have a strong preference for $\mathrm{H} 3$ and $\mathrm{H} 4$, while nucleoplasmin (Earnshaw et al., 1980) and the histone chaperone Nap1 (Ito et al., 1996; Mosammaparast et al., 2001) favor binding to $\mathrm{H} 2 \mathrm{~A}$ and $\mathrm{H} 2 \mathrm{~B}$. For the latter, a role in the nuclear transport of the core histones H2A and H2B was meanwhile established (Mosammaparast et al., 2005; Mosammaparast et al., 2002a). Since Nap1 binds to the amino-terminal tails of the core histones $\mathrm{H} 2 \mathrm{~A}$ and $\mathrm{H} 2 \mathrm{~B}$, an involvement in the nuclear accumulation of globular domains can certainly be excluded. However, the recent studies convincingly demonstrate that a complex consisting of the import receptor Kap114p, H2A and H2B, and Nap1 is present in the nucleus. Moreover, the presence of this complex is specifically promoted by Nap1, whose function (histone deposition) can be modulated by Kap114p (Mosammaparast et al, 2005). 


\subsubsection{Tales about tails: amino-terminal domains as import signals}

In accordance with the studies in yeast (Mosammaparast et al., 2002b; Mosammaparast et al., 2001) we showed that also in mammals the amino-terminal tails of the core histones contain ncNLSs (Fig.23). Although histones are generally highly conserved among eukaryotes, yeast histones and mammalian histones are different.

\author{
H 2 A / d human \\ H2A. 1_yeast \\ H 2 A / d human \\ H2 A. 1_yeast \\ H 2 A / d human \\ H 2 A. 1_yeast
}

H 2 B / d_human H 2 B. 1_yeast

H 2 B / d human H 2 B . 1_yeast

H 2 B / d human H 2 B . 1_yeast

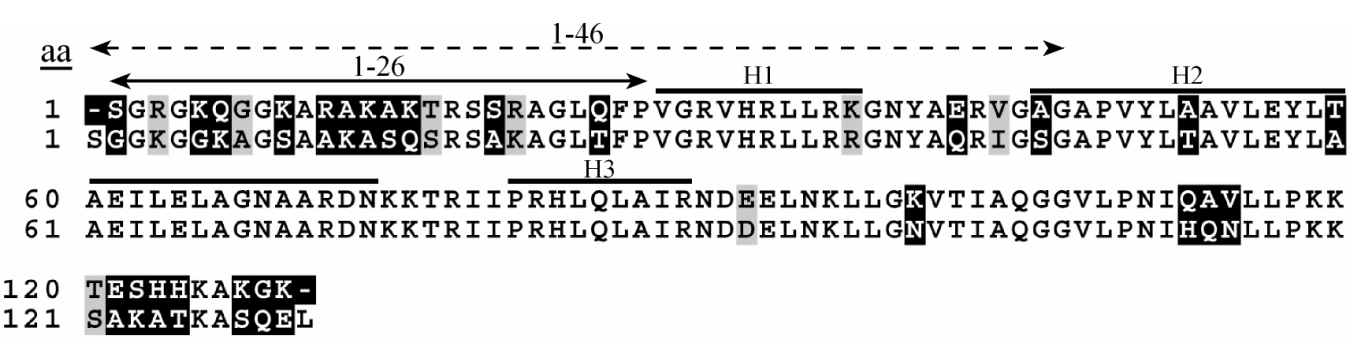

H3/k_human H3_yēast

H3 / k human H3_yēast

H 3 / k human H3_yeast

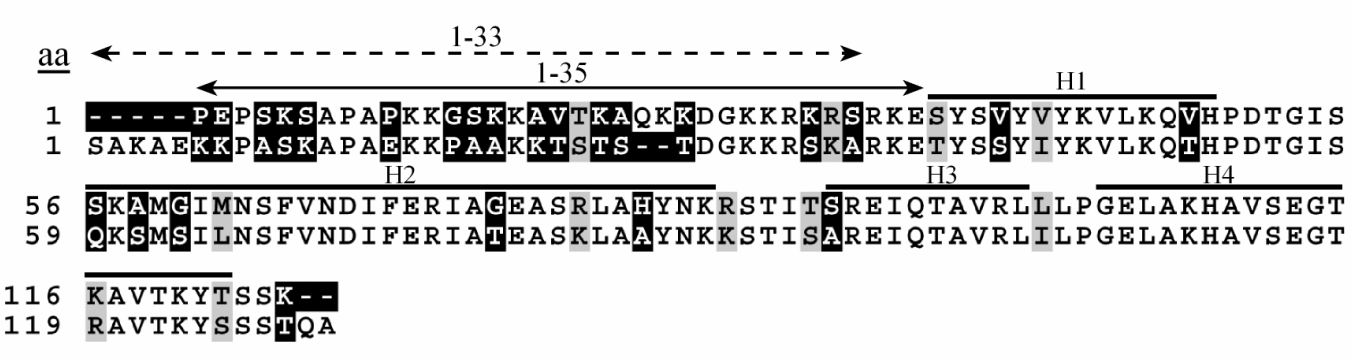

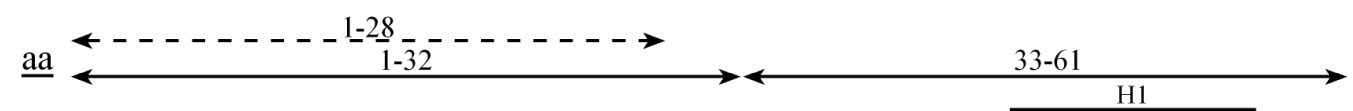

1 ARTKQTARKSTGGKAPRKQLATKAARKSAPATGGVKKPHRYRPGTVALREIRRYQKSTEL 1 ARTKQTARKSTGGKAPRKQLASKAARKSAPSTGGVKKPHRYKPGTVALREIRRFQKSTEL 61 LIRKLPFQRLVREIAQDFK TDLRFQS SAVMALQEACEAYLVGLFEDTNLCAIHAKRVTIM 61 LIRKLPFQRLVREIAQDFKTDLRFQSSAIGALQESVEAYLVSLFEDTNLAAIHAKRVTIQ H4

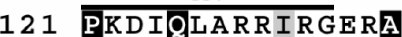

121 KKDIKLARRLRGERS

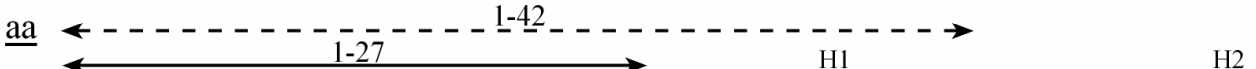

1 SGRGKGGKGLGKGGAKRHRKVLRDNIQGITKPAIRRLARRGGVKRISGIIYEETRGVLKV

1 SGRGKGGKGLGKGGAKRHRKILRDNIQGITKPAIRRLARRGGVKRISGLIYEEVRAVLKS $\mathrm{H} 3$

61 FLENVIRDAVTYTEHAKRKTVTAMDVVYALKRQGRTLYGFGG

61 FLESVIRDSVTYTEHAKRKTVTSLDVVYALKRQGRTLYGFGG

FIG. 23. Sequence alignment of core histones in lower and higher eukaryotes. Sequences of yeast and human core histones are aligned. Although histones are highly conserved among eukaryotes, yeast histones are most divergent from mammalian histones. Non-conserved amino acid residues are indicated by a black background, whereas similar amino acid residues are shaded in gray (aa, position of the amino acid in the respective organism). The solid (human) and dashed (yeast) arrows on top of the sequence alignments correspond to the regions essential for nuclear accumulation of the different core histones as previously mapped (Baake et al., 2001b; Mosammaparast et al., 2002b; Mosammaparast et al., 2001). The black bars on top of the sequence alignments represent the different $\alpha$-helices in the secondary structure (histone fold motif) as observed in the X-ray structure (Luger et al., 1997). 
In particular, strong differences are obvious in the amino-terminal tails of the core histones $\mathrm{H} 2 \mathrm{~A}$ and H2B (Fig.23). Despite their evolutionary distance, however, in both species the aminoterminal tails were unambiguously identified as regions which are necessary and sufficient to confer nuclear accumulation. Furthermore, different signals are recognized by similar import receptors in yeast and in mammals. The nuclear import of core histones occurs along redundant pathways, which means that the identified import receptors to some extent can substitute for each other. Hence, the question arises what characterizes the NLSs in the amino-terminal tails of the core histones? Comparing the ncNLSs in the amino-terminal tails of the core histones with each other and with other ncNLSs such as the IBB domain (Gorlich et al., 1996a; Weis et al., 1996b) and the BIB domain (Jakel and Gorlich, 1998) no obvious sequence similarity can be observed (Fig.24). However, one feature all the different import signals have in common is the large number of basic amino acid residues (lysine and arginine) in the sequence (H2A: 8/26aa (31\%); H2B: 15/35aa (43\%); H3: 18/61aa (30\%); H4: 9/27aa (33\%); BIB: 17/43aa (40\%); IBB: 16/44aa (36\%)). Thus, it appears that a certain number of positively charged amino acids per total number of amino acids contributing to the nuclear targeting function may be required to be recognized by the rather acidic import receptors.
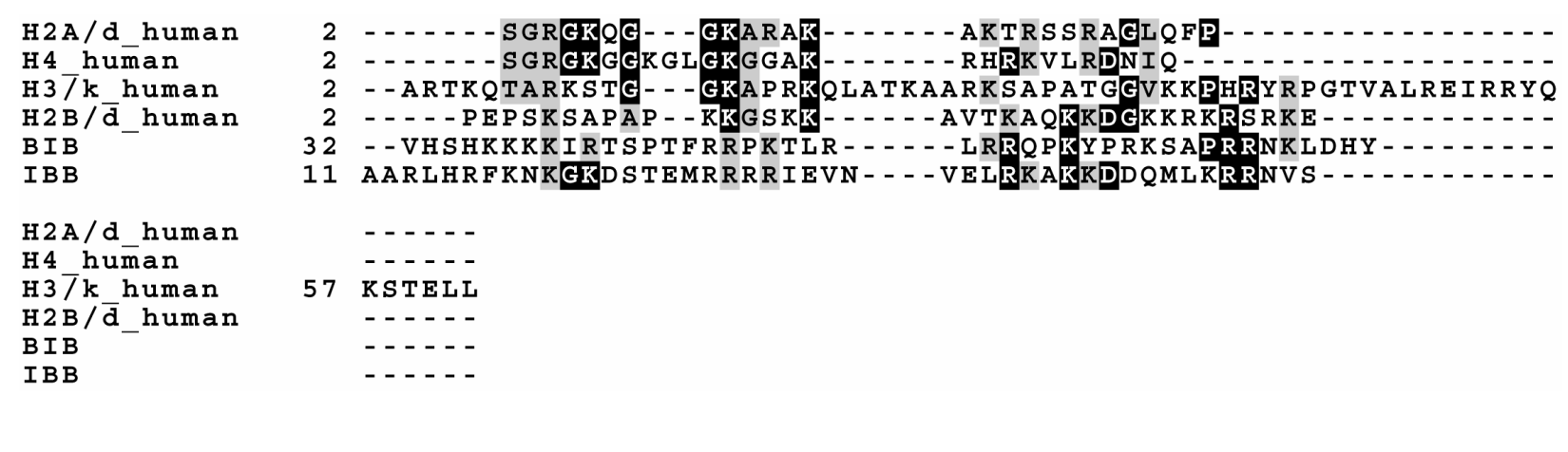

FIG. 24. Sequence alignment of non-classical NLSs (part one). Sequences of the amino-terminal tails of the human core histones $\mathrm{H} 2 \mathrm{~A}, \mathrm{H} 2 \mathrm{~B}, \mathrm{H} 3$, and $\mathrm{H} 4$, the IBB domain of importin $\alpha$, and the BIB domain of the ribosomal protein L23 are aligned. Apart from the high amount of positively charged amino acid residues the different non-classical NLSs lack a strong homology. Identical amino acids are indicated by a black background whereas similar amino acid residues are shaded in gray (positions of the amino acid residues in the respective sequences are indicated by the numbers).

Common features of importin $\beta$ family members include (i) an amino-terminal RanGTP-binding domain (ii) a similar large size, and (iii) an acidic isoelectric point (4.6-5.9) (Gorlich et al., 1997). From the crystal structure of importin $\beta$ bound to the IBB domain of importin $\alpha$ (Cingolani et al., 1999), it is known that several positively charged amino acid residues of the 
(NLS) sequence are engaged in ionic interactions with aspartic and glutamic acid residues that line the inner $B$ helices of importin $\beta$. In addition to the electrostatic interactions on the surface of the molecule also hydrophobic interactions (e.g. aliphatic portions of the side chains of positively charged amino acids are involved in hydrophobic interactions with tryptophan residues of the importin) are involved and result in the binding of positively charged amino acids with high affinity and specificity. The positions of these structural determinants on the surface of the importin determine the required spacing of essential lysine and arginine residues in the sequence of the transport cargo. Hence, besides the fact that cargo binding involves the specific (chemical) recognition of key residues, a large number of positively charged amino acid residues in the sequence of potential cargo molecules certainly increases the probability to meet the requirements given by the transport receptors.

The ncNLSs of the core histones (amino-terminal tails) and the ribosomal protein L23a (BIB domain) are recognized by essentially the same import receptors (importin $\beta$, importin 5, importin 7, and transportin). Comparison of the amino acid sequences of these import signals reveals the following consensus motif: $(K / R) X_{3-4}(K) X_{3}(K) X_{3-4}(K / R) X_{2}(K / R)$. However, the amino-terminal tails of the histones $\mathrm{H} 2 \mathrm{~A}$ and $\mathrm{H} 2 \mathrm{~B}$ in yeast do not contain such a motif because of the evolutionary divergence. In addition, a shorter fragment of the BIB domain (amino acids 52 to 74), which still contains the identified consensus motif, almost completely lacks an importin binding capacity (Jakel and Gorlich, 1998). Hence, the identified motif does not represent a consensus for ncNLSs which can be recognized by several importins but most likely originated accidentally due to the high abundance of positively charged amino acid residues in the signal regions. The apparent lack of a unique consensus for importin $\alpha$-independent cargoes (ncNLSs) perhaps can be explained with the existence of numerous interactions between importin side chains and the main chain of the different signal sequences, as observed for the binding of the ncNLS of the parathyroid hormone-related protein (PTHrP) to importin $\beta$ (Cingolani et al., 2002). This would explain why several different signal sequences can be accommodated in the binding groove of import factors with high affinity but without strict conservation of sequence.

\subsubsection{Binding sites in importin $\beta$}

Having shown that the amino-terminal tails of the core histones contain ncNLSs which are recognized by several import receptors, we finally asked, what are the binding sites in the transport receptors for the core histone import signals? To address this question, we wanted to characterize the binding sites in importin $\beta$ for the core histone H2B. In an oversimplified picture 
two cargo binding sites exist on the inner concave surface of importin $\beta$. The cargo binding site I (or IBB binding domain) consists of the HEAT repeats 7 to 19 (Cingolani et al., 1999), while the second cargo binding site in importin $\beta$ includes the HEAT repeats 1 to 11 (Cingolani et al., 2002) which entirely overlaps with the RanGTP binding domain (HEAT 1-10) (Vetter et al., 1999). This of course implies that cargoes binding to the amino-terminal cargo binding site II must be released by RanGTP via a direct competition for the same binding site. In contrast, cargoes binding to the cargo binding site I of importin $\beta$ are released by RanGTP due to a conformational change in importin $\beta$ (Cingolani et al., 2000). However, we wanted to examine which binding site $\mathrm{H} 2 \mathrm{~B}$ uses in importin $\beta$ and therefore performed in vitro binding assays. In addition to immobilized H2B, either the BIB domain of rpL23a or the IBB domain of importin $\alpha$ were used as potential second cargo of importin $\beta$ to investigate whether simultaneous binding of $\mathrm{H} 2 \mathrm{~B}$ and IBB (H2B and BIB) to importin $\beta$ can occur. Since it is known that BIB domain and IBB domain bind to the cargo binding site I, simultaneous binding would have indicated that H2B uses the cargo binding site II on importin $\beta$. The results of these preliminary studies demonstrated that binding of importin $\beta$ to $\mathrm{H} 2 \mathrm{~B}$ was competed by addition of either BIB or IBB domain suggesting that the amino-terminal tails of core histones bind also to the cargo binding site I on importin $\beta$. This is in agreement with Muhlhausser et al. who had reported that in vitro nuclear uptake of core histones could be completely inhibited by addition of an excess of BIB domain (Muhlhausser et al., 2001). Thus, the parathyroid hormone-related protein most likely remains the only substrate which is known to bind to the second cargo binding site on importin $\beta$.

\subsection{Nuclear transport of NF-Y: one complex, dual mechanism}

In eukaryotes, protein encoding genes are transcribed by RNA polymerase II under the control of short DNA elements located in promoters and enhancers which are recognized by the general transcription machinery and transacting factors (for review, see Lemon and Tjian, 2000; Workman and Kingston, 1998). Statistical analysis revealed that the CCAAT box is one of the most ubiquitous promoter elements, being present in about $30 \%-65 \%$ of eukaryotic promoters, ranging from yeast to mammals (Bucher, 1990; Suzuki et al., 2001). The key role of the CCAAT-specific transcription factor NF-Y is to act synergistically with other factors for activation (reviewed in Maity and de Crombrugghe, 1998). In mammals, genes relying on CCAAT elements and therefore controlled by NF-Y include tissue specific (Berry et al., 1992; Ronchi et al., 1996), inducible (Marziali et al., 1997; Roy and Lee, 1995), and cell cycle regulated genes (Caretti et al., 2003) whereas housekeeping genes generally do not rely on 
CCAAT boxes (Mantovani, 1998; Mantovani, 1999). Due to the important functional roles of NF-Y in cell cycle regulation and aging, characterization of the underlying nuclear transport mechanisms of NF-Y was important and potentially significant.

NF-Y is a heterotrimeric complex composed of three subunits, NF-YA, NF-YB, and NF-YC which are all required for CCAAT binding (McNabb et al., 1995; Sinha et al., 1995). Here, we report that in higher eukaryotes the subunits of NF-Y are imported into the nucleus by distinct pathways. Importin $\beta$ mediates nuclear import of NF-YA, whereas dimerization of the two HFM containing subunits NF-YB and NF-YC is a prerequisite for nuclear accumulation conferred by importin 13.

\subsubsection{Nuclear import of NF-YB and NF-YC depends on dimerization}

In contrast to the so far known import and export cargoes of importin 13, i.e. the SUMOconjugating enzyme hUBC9, RBM8 (Y14) (either alone or with MGN), translation initiation factor eIF1A (Mingot et al., 2001) and paired-type homeodomain transcription factors Pax6, Pax3, and Crx (Ploski et al., 2004), the NF-YB/NF-YC dimer represents, to our knowledge, the first example of an importin 13 specific substrate the recognition of which solely depends on complex formation. Indeed it has been previously reported that the NF-YB like protein (YBL1) binds to immobilized importin 13 (Mingot et al., 2001), however, it is unknown whether YBL1 was bound to importin 13 as monomeric subunit or as dimeric complex with its histone fold partner YCL1 (Bolognese et al., 2000). By mass spectrometry, Mingot et al. identified only a few interacting proteins fished from the cytosolic HeLa cell extract, from which hUBC9 and RBM8 (Y14) have been verified as import cargoes and eIF1A as export cargo (Mingot et al., 2001). Despite their close relation to NF-YB and NF-YC, YBL1 and YCL1 are divergent with respect to several functions. For instance, the YBL1/YCL1 dimer is incapable of interacting with NF-YA since essential binding sites in the HFM dimer are obviously not conserved (Bolognese et al., 2000). Furthermore, no cross-heterodimerization with NF-YB and NF-YC (NF-YB-YCL1 and NF-YC-YBL1 interactions) could be observed, and unlike NF-YB and NF-YC, which can interact with TBP (Bellorini et al., 1997), the YBL1/YCL1 dimer shows a lack of TBP-TATA interaction. However, despite clear divergence in functional terms, NF-YB-NF-YC and YBL1-YCL1 have coevolved the capacity to interact with nucleosomal structures (Bolognese et al., 2000).

Binding of importin 13 to the NF-YB/NF-YC dimer is controlled by the RanGTPase system in the typical importin-like fashion, i.e. the NF-YB/NF-YC complex can bind importin 13 at low 
RanGTP levels in the cytoplasm and becomes displaced upon RanGTP binding in the nucleus. Despite the specific binding of importin 13 to the NF-YB/NF-YC dimer, the data in Fig.4B showing that strong over-expression of NF-YB did not significantly alter its subcellular distribution as it was observed for NF-YC also allow another interpretation. This alternative model would suggest that NF-YB contains a NLS which is exposed upon NF-YC binding and enhances the binding of importin 13 to NF-YB. Can this model be excluded? In fact, our in vitro binding studies (Fig.11) and in vitro nuclear import assays (Fig.10C) clearly show that only the NF-YB/NF-YC dimer, but not the monomeric components, are recognized by importin 13 (or any other importin) and are imported into the nucleus of permeabilized cells. Hence, we conclude that neither NF-YB nor NF-YC does contain a functional NLS and suppose that a distinct binding platform for importin 13 interactions derives from dimerization of the two HFM containing subunits NF-YB and NF-YC. In this context, binding of importin 13 to the NF-YB-NF-YC dimer mimics NF-YA which is also not able to interact with either NF-YB or NF-YC alone (Kim et al., 1996b; Sinha et al., 1996; Sinha et al., 1995).

Further, nuclear transport of the three NF-Y subunits was characterized and compared in Xenopus oocytes. The microinjection experiments clearly showed that the kinetics of nuclear translocation differ among the subunits. While NF-YA was imported rapidly into the nucleus of Xenopus oocytes, NF-YB and NF-YC were imported much slower. Again, we explain the different import kinetics with the requirement for NF-YB and NF-YC to interact with the endogenous histone fold partner to be translocated into the nucleus mediated by importin 13 . A prerequisite for this assumption was that an orthologue of importin 13 can also be found in Xenopus laevis. Therefore, we used the human importin 13 sequence to search the sequence database of Xenopus laevis (http://www.sanger.ac.uk/cgi-bin/blast/submitblast/x_tropicalis). We found an EST (cDNA clone image: 6634964) with high homology (sequence identity: $74 \%$, protein identity: $83 \%$ ) to the human importin 13 suggesting that an orthologue of importin 13 exists in Xenopus laevis. Also in this context, Mingot et al. reported about putative orthologues of importin 13 in representative branches of eukaryotes, such as plants, insects, nematodes and fungi, which suggests that importin 13 evolved quite early in eukaryotic phylogenesis (Mingot et al., 2001).

The subcellular localization of endogenous NF-Y subunits and differently tagged, overexpressed versions of the three subunits was examined in HeLa cells. While the subcellular localization of endogenous NF-Y subunits was detected with appropriate antibodies, HeLa cells were also transiently transfected with plasmid DNA encoding the three differently tagged subunits. Besides $ß$-galactosidase (ß-gal), the enhanced green fluorescent protein (EGFP), 
hemagglutinin (HA), and the myc-tag was used as reporter gene. The observed differences regarding the subcellular localization of the differently tagged NF-YB and NF-YC fusion constructs (proteins) reflects abundance. The $\beta$-gal tagged fusion constructs were under control of a SV40 promoter causing moderate over-expression of the $\beta$ - gal fusion proteins, whereas the EGFP-, HA-, and myc-tagged fusion proteins were strongly over-expressed in HeLa cells because their genes were under control of a CMV promoter. In view of that, to identify the regions essential for the nuclear localization of NF-YB and NF-YC, fragments of each HFM containing subunit were only moderately ( $\beta$-gal fusion protein) over-expressed in HeLa cells (in the presence of corresponding endogenous histone fold partner). Thereby it was shown that the minimal sequence regions essential for the nuclear localization of NF-YB and NF-YC correspond to their histone fold motifs. Since only the NF-YB/NF-YC dimer, but not the monomeric components, are translocated into the nucleus in an importin 13 dependent manner, the in vivo transfection experiments can also be considered as first attempt to map the binding sites in the NF-YB/NF-YC complex for importin 13. Hence, the results showing that the histone fold motifs match the sequence region necessary for the nuclear localization of NF-YB and NF-YC also indicate that the binding site in the NF-YB/NF-YC dimer for importin 13 is located within the dimerized histone fold motifs. This assumption is further supported by the fact that importin 13 binding to the NF-YB/NF-YC complex was competed by NF-YA. The addition of increasing amounts of NF-YA caused a steady reduction of importin 13 binding, whereas binding of importin 13 to the NF-YB/NF-YC dimer was completely abolished by an excess of NF-YA. Further, the results clearly showed that NF-YA has a higher affinity than importin 13 for the NF-YB/NF-YC dimer. Thus, NF-YA may contribute to the release of the HFM containing subunits from importin 13 in the nucleus. Consistent with other importins, the dissociation of the NF-YB/NF-YC dimer from importin 13 is dependent on RanGTP. However, we could show that NF-YA directly competes with importin 13 for binding to the NF-YB/NF-YC dimer. In other words, NF-YA stimulates this dissociation of NF-YB/NF-YC from importin 13 and therefore may be necessary at lower RanGTP concentrations.

It is known that in NF-YB two conserved amino acids in helix $\alpha 2$ (E90 and S97) and in NF-YC several residues in both helices $\alpha 1$ and $\alpha \mathrm{C}$ are required for NF-YA association (Kim et al., 1996b; Romier et al., 2003; Sinha et al., 1996; Xing et al., 1993). Whether these three elements of secondary structure that form a groove where NF-YA is supposed to bind are also recognized by importin 13 is not known, but further studies will surely address this question. Additionally, clusters of basic amino acid residues created by heterodimerization of the histone fold motifs may promote the binding of importin 13. However, like H2A/H2B (Luger et al., 1997) and 
NC2 $\alpha / \mathrm{NC} 2 \beta$ (Kamada et al., 2001) the calculated electrostatic potential of the upper surface of the histone-like portion (HFM dimer) of the NF-YB/NF-YC complex is highly basic. Thus, this region of positive electrostatic potential certainly permits favorable polar interactions with the negatively charged nucleic acid backbones and conceivably also with negatively charged portions of importin 13.

\subsubsection{Nuclear import of NF-YA: monomeric, but regulated}

Initially, to roughly characterize the pathway involved in the nuclear import of NF-YA, we performed in vitro import assays in the presence of non-fluorescent core histones as potential import competitors. The addition of an excess of unlabeled core histones completely blocked the nuclear import of NF-YA which indicated that at least one of the transport receptors mediating nuclear import of core histones (importin $\beta$, importin 5, importin 7, importin 9, and transportin) was also required for the nuclear uptake of NF-YA. In contrast, nuclear import of the NF-YB/NF-YC dimer mediated by importin 13 was not challenged by the addition of core histones, but by NF-YA. Addition of NF-YA led to a reduced nuclear import of the NF-YB/NF-YC complex in the presence of importin 13 and proved again that only the histone fold dimer can be recognized by importin 13 and not the heterotrimeric NF-Y holocomplex, or in other words that NF-YA competes with importin 13 for binding to the NF-YB/NF-YC dimer.

Regarding nuclear targeting signals, the functional NLS of NF-YA is located in the evolutionarily conserved domain at the C-terminus of the protein including the subunit interaction domain and DNA binding domain. Overlap of DNA binding region and NLS may help to release of NF-YA from its import receptor (importin $\beta$ ) after nuclear translocation. However, specific DNA binding has not been observed for NF-YA but only for the trimeric NF-Y complex (Mantovani, 1999). The NLS in NF-YA can by clearly considered as nonclassical type because: (i) the sequence is much to long for a classical signal which consists of short stretches of positively charged amino acids, and (ii) members of the importin $\beta$ family directly bind (importin $\alpha$-independent) to NF-YA. The non-classical NLS (ncNLS) of NF-YA consists of up to 56 amino acids (not further characterized by $\mathrm{N}$ - or C-terminal deletions) including 11 arginine and seven lysine residues. Mutation of six out of the 18 basic amino acid residues within that stretch caused a nearly complete loss of nuclear accumulation (in vivo transfection experiments) indicating that a certain number of positively charged amino acid residues in the NLS region of NF-YA are required for nuclear targeting. The results of the mutagenesis screen were confirmed by in vitro binding studies with mutant NF-YA fusion proteins, and further supported our conclusion. Instead of applying a more comprehensive 
mutagenesis study, in the presented random mutagenesis screen we focused on just altering basic amino acid residues for three reasons: (i) to generally confirm the presence of the ncNLS in this sequence region, (ii) to elucidate and to show the importance of the high number of basic amino acid residues for the functionality of the mapped ncNLS, and (iii) to clearly discriminate between an arginine/lysine rich ncNLS type and the M9 domain (Michael et al., 1995) type, which is not enriched in basic amino acid residues but instead comprises many aromatic residues and is rich in glycine.

If we compare the NLS of NF-YA to other ncNLSs such as the IBB domain, BIB domain, and the amino-terminal tails of core histones, no obvious sequence similarity can be observed (Fig.25; see also Fig.24 again). Strikingly similar between these different import signals, however, is the large number of basic amino acid residues in the sequence (lysine and arginine residues comprise more than $30 \%$ ).

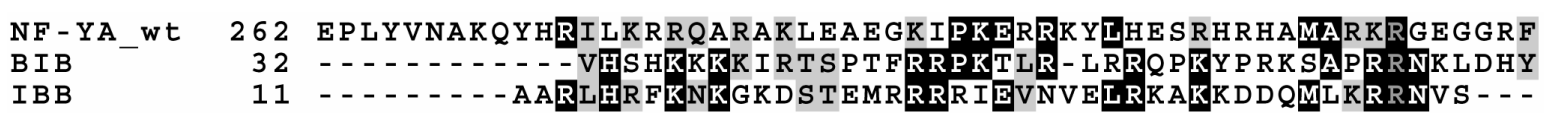

FIG. 25. Sequence alignment of non-classical NLSs (part two). Sequences of the ncNLS of NF-YA, the IBB domain of importin $\alpha$, and the BIB domain of the ribosomal protein L23 are aligned. Similarity between these different import signals is the large number of positively charged amino acid residues (lysine and arginine) in the sequence. Identical amino acids are indicated by a black background while similar amino acid residues are shaded in gray (positions of the amino acid residues in the respective sequences are indicated by the numbers).

Hence, in the cases mentioned above a specific number of positively charged amino acids per total number of amino acids contributing to the nuclear targeting function may be required to be recognized by the rather acidic import receptors (see also chapter 4.1.4). The fact that random mutagenesis of six basic amino acid residues in the NLS region of NF-YA (Fig.8B, pJK130, pJK131) nearly blocks nuclear uptake, but deletion of the linker region in the middle of the ncNLS (16aa including also six basic amino acid residues) did not change the nuclear localization of the $\beta$-galactosidase fusion protein (Fig.7, pJK87), supports this assumption. Why? Mutagenesis left the total number of amino acids unchanged (56aa), but significantly reduced the positive charge of the sequence region (12 basic aa/56aa correspond to only $21 \%$ instead of 18 basic aa/56aa corresponding to $32 \%$ ). In contrast, deletion of the linker region localized between the subunit interaction domain (BC) and the DNA binding domain (DNA) reduced the total number of amino acids (from 56aa to 40aa), but left the overall positive charge of the sequence region basically unchanged (12 basic aa/40aa corresponds to about $30 \%$ ), and perhaps therefore 
the NLS can still be recognized by import receptors. As said before, a large number of positively charged amino acids in the sequence of potential cargo molecules certainly increase the probability to meet the requirements given by the transport receptors, eventually leading to cargo binding.

As mentioned, the greater complexity compared with classical NLSs and very basic nature of the ncNLS of NF-YA reminds one of the BIB domain of rpL23a that consists of 43 amino acids including nine arginine and eight lysine residues (Jakel and Gorlich, 1998). The BIB domain, which might be considered as an archetypical import signal, specifically binds to importin $\beta$, importin 5, importin 7 or transportin, respectively. We observed the same for NF-YA. However, in contrast to the BIB domain (and the amino-terminal tails of the core histones), binding of the last three import factors to NF-YA was not sufficient to confer nuclear uptake in vitro. NF-YA was only imported into the nuclei of permeabilized cells when importin $\beta$ together with a Ran mix and an energy-regenerating system was present. Nevertheless, we do not exclude that the other three import factors also mediate nuclear transport of NF-YA in vivo which perhaps could not be reconstituted because of lower affinity towards the cargo and the experimental conditions chosen. Two additional aspects should be considered: Firstly, a weaker affinity of a NLS for other importins probably allows transport receptors to back up each other, which for instance might help cells to respond to particularly high transport demands. Secondly, to further help evaluating the fact that importin $\beta$ has the highest NF-YA binding competence, affinity measurements should be definitely performed. Whether the ncNLS of NF-YA located in the evolutionarily conserved C-terminal region of the protein mimics the BIB domain to enter the nucleus and whether core histones use a similar strategy can not be answered and are questions for future studies. For instance, the binding site in importin $\beta$ for the ncNLS of NF-YA could be mapped and also competition binding studies (as for $\mathrm{H} 2 \mathrm{~B}$, see chapter 4.1.5) could be put forward to identify substrates utilizing the same binding sites in importin $\beta$.

One way to control interactions between cargoes and transport receptors is to mask the transport signal. Sequence masking/unmasking can be achieved by posttranslational modification of the transport cargo, it can involve the degradation or dissociation of an inhibitory protein or it can be due to association with another protein. Numerous studies showed that the most frequent posttranslational modification to regulate interactions between targeting sequences in cargoes and transport receptors is phosphorylation/dephosphorylation (see also chapter 1.7). The phosphorylation sites are often found in close vicinity to the targeting signal and phosphorylation itself then either stimulates or inhibits its function. However, based on our studies and in contrast to the nuclear transport of many specific transcription factors, nuclear uptake of NF-YA appears 
not to be additionally regulated by posttranslational modifications (and therefore rather resembles the behavior of general transcription factors). Anyway, the greater complexity of large non-classical NLSs (as identified in NF-YA) compared to short classical NLSs suggests that classical signals will be much easier to modulate by posttranslational modifications. In other words, functional modifications of non-classical signals by phosphorylation are rather unlikely.

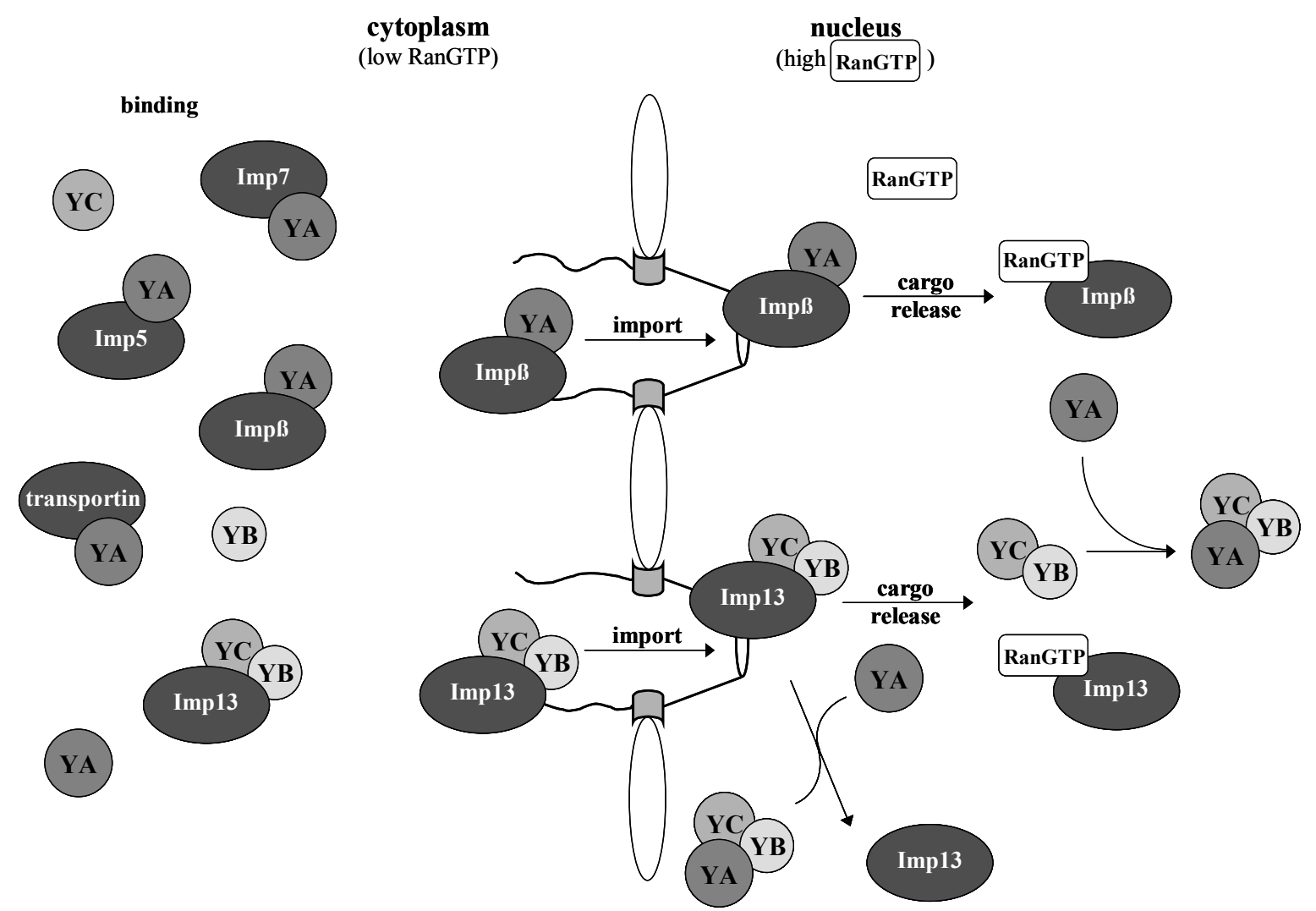

FIG. 26. Proposed model for the nuclear transport of NF-Y subunits in higher eukaryotes. In higher eukaryotes the subunits of NF-Y are imported into the nucleus by distinct pathways. Importin $\beta$ mediates nuclear import of NF-YA, whereas dimerization of the two HFM containing subunits NF-YB and NF-YC is a prerequisite for nuclear accumulation conferred by importin 13. NF-YA competes with importin 13 for binding to the NF-YB/NF-YC dimer and therefore may aid the release of the HFM containing subunits from importin 13 in the nucleus. See discussion (chapter 4.2) for details.

Since the identified NLS of NF-YA also contains its subunit interaction domain, indicating that the respective amino acid residues are of equal importance for subunit interaction and importin binding, it was of interest to find out whether NF-YA can still bind import receptors (particularly importin $\beta$ ) once it is assembled into the NF-Y holocomplex. The results of GST pulldown experiments showed that NF-YA does not suffice to mediate the interaction of importin $\beta$ with the functional NF-Y holocomplex. Hence, our data suggest that binding and subsequent nuclear 
transport mediated by importin $\beta$ is restricted to the single NF-YA subunit and can not be applied to the trimeric NF-Y complex. This, however, implies that in the assembled trimeric complex the NLSs are apparently inaccessible so that the complex can not be imported by the transport receptors that import either NF-YA or the NF-YB/NF-YC dimer (remember NF-YA competes with importin 13 for binding to the NF-YB/NF-YC dimer). Hence, trimeric complexes which most likely also assemble in the cytoplasm would remain trapped there, unable to fulfill their function of transcriptional activators. Whether this (possible) cytoplasmic retention serves some physiological purpose or not, we don't know. However, the in vitro pre-assembled NF-Y complex consisted of subunits carrying GST-tags that might have interfered with importin binding. Thus, it can not be completely excluded that NF-YA can mediate the interaction of importin $\beta$ with an untagged NF-Y holocomplex in vivo. On the other hand, we have shown that ncNLS and subunit interaction domain of NF-YA overlap.

\subsubsection{Different kingdoms, different rules}

The nuclear import of Aspergillus nidulans CCAAT binding factor AnCF, the fungal homologue of the evolutionarily conserved heterotrimeric NF-Y complex, recently has been studied in this filamentous fungus. AnCF consists of the three subunits HapB (NF-YA homologue), HapC (NF-YB homologue), and HapE (NF-YC homologue). The mode of protein interactions between the three NF-Y subunits appears to be similar in higher and lower eukaryotes. In detail, the HFM containing subunits (NF-YB and NF-YC) dimerize tightly head to tail via their HFMs, which offers a complex surface required for association of the third subunit (NF-YA). In HapB (NF-YA homologue) a functional NLS in the C-terminus of the protein was identified outside of the evolutionarily conserved domain (Steidl et al., 2004). In contrast, we showed that in mammals NF-YA contains an ncNLS in the 56 amino acid long evolutionarily conserved region (Fig.7). In analogy to our findings in the human homologues NF-YB and NF-YC, no NLSs were identified in the HFM containing subunits HapC and HapE. Based on the data that $\Delta \mathrm{HapB}$ strains are viable but the subcellular localization of HapC and HapE is cytoplasmic, and based on the fact that neither HapC-EGFP (in the absence of HapE or HapB) nor HapE-EGFP (in the absence of HapC or HapB) entered the nucleus, the authors concluded that the trimeric AnCF complex is transported to the nucleus via the NLS in HapB (Steidl et al., 2004). Since the identified NLS in HapB (amino acid 326-KRK) is not present in higher eukaryotes (Fig.27) the proposed piggyback transport of the two HFM containing subunits via HapB as pre-assembled trimeric complex can not be generally applied but appears to be rather unique for the Aspergillus nidulans CCAAT-binding complex AnCF. 


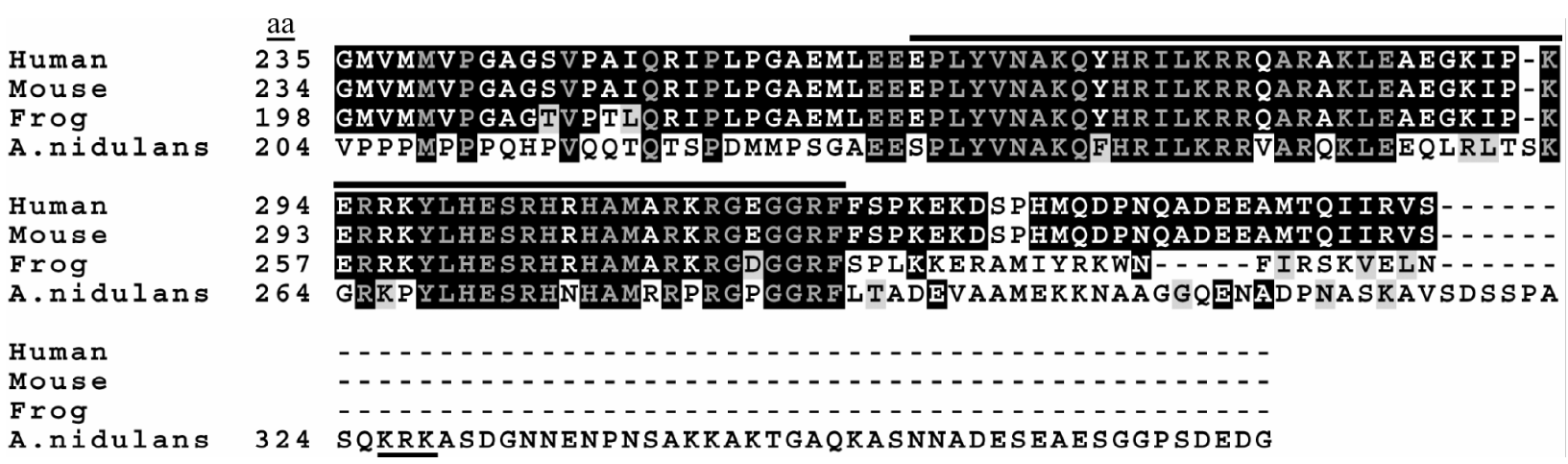

FIG. 27. Partial sequence alignment of NF-YA. Sequence alignment of the C-terminal end of NF-YA from human, Mus musculus, Xenopus laevis, and Aspergillus nidulans. Identical amino acids are indicated by a black background, whereas conserved amino acids (identical residues in all sequences) are shaded in gray additionally (aa, position of the amino acid in the respective organism). The bar on top of the sequence alignment represents the ncNLS of NF-YA which corresponds to the evolutionarily conserved region (for mapping and characterization of the ncNLS of NF-YA, see Fig.7 and Fig.8). The basic amino acid residues 326-328 (KRK, additionally underlined) are essential for the nuclear localization of HapB (NF-YA homologue) in Aspergillus nidulans (Steidl et al., 2004).

Further, it is worth to mention that we were unable to retrieve bona fide importin 13 genes from Aspergillus nidulans ESTs which strongly indicates that a putative importin 13 orthologue is not present in Aspergillus nidulans. This fact, however, may additionally explain why in this filamentous fungus nuclear transport of the two HFM containing subunits HapC and HapE (NF-YB and NF-YC homologues) entirely depends on the third subunit HapB (NF-YA homologue). In addition, the physiological significance of NF-Y seems to be different in higher and lower eukaryotes. Aspergillus nidulans lacking HapB ( $\Delta$ hapB strain) showed a phenotype of slow growth, poor conidiation, and reduced expression of genes containing a CCAAT box (Steidl et al., 1999; Steidl et al., 2004), but was viable, whereas in higher eukaryotes NF-Y mediated transcription is essential for cell proliferation and viability. This was for instance demonstrated by a conditional deletion of both NF-YA alleles in primary cultures of mouse embryonic fibroblast cells which led to a complete block in cell cycle progression and subsequent initiation of apoptosis (Bhattacharya et al., 2003).

\subsubsection{NF-YB and NF-YC fulfill nuclear functions independent of NF-YA}

In general, NF-Y is required to organize the chromatin in proximity of transcriptional start sites thereby enabling recruitment of co-activators (Li et al., 1998; Mantovani, 1999). In the literature there are contradictory statements regarding the nature of NF-Y interactions with cell cycle (regulated) promoters, varying from highly dynamic (Caretti et al., 2003) to totally constant 
through the cell cycle (Tommasi and Pfeifer, 1995; Zhu et al., 2004). In this context, Frontini et al. recently showed that the nuclear localization of NF-YC is cell cycle regulated and depends on the presence of its histone fold partner NF-YB (Frontini et al., 2004). The results of our co-expression studies are in accordance with these observations and indicate that NF-YB and NF-YC mediate nuclear localization of its corresponding histone fold partner (Fig.4C). Frontini et al. interpreted the cytoplasmic localization of strongly over-expressed NF-YC by a mechanism in which NF-YC first enters the nucleus and is then (when in excess with respect to NF-YB heterodimerization capacity) exported to the cytoplasm via a CRM1-dependent pathway (Frontini et al., 2004). However, our data did not confirm this. The results of microinjection experiments in Xenopus oocytes (export studies, Fig.5A) and the results of transient transfections of HA-NF-YC in HeLa cells (LMB treatment, Fig.5C) rather indicate that the cytoplasmic localization of strongly over-expressed NF-YC was not due to nuclear export but due to the lack of an independent NLS in NF-YC. Hence, our results point to a scenario where NF-YC has to dimerize with NF-YB (and vice versa) to be imported into the nucleus. Since dimerization of the HFM containing subunits depends on a reduced form of NF-YB (Nakshatri et al., 1996) the cellular redox environment of mammalian cells might regulate the nuclear levels of NF-YC.

Additionally, to support their standpoint, Frontini et al. presented data showing that the overexpressed NF-YC splicing isoform, named NF-YCb (CBF-Cb), was not restricted to the cytoplasmic compartment but localized in the nucleus. In comparison to NF-YC the splicing isoform NF-YCb lacks the amino acid residues 36 to 97 coding for the helices $\alpha 1$ and $\alpha 2$ of the HFM (Chen et al., 2002; Romier et al., 2003). Based on these results, Frontini et al. argue that the amino acid residues 36-97 of NF-YC (which are essential for NF-YB interactions) contain a leucine-rich NES (Frontini et al., 2004). However, looking at the amino acid sequence of NF-YC there are only two stretches (26-MEIRNLTT $\underline{V}, 107-\underline{F D Q F D F L I D I})$ that match the leucine-rich NES consensus sequence: $\Phi X_{2-3} \Phi X_{2-3} \Phi X \Phi(\Phi=\mathrm{L}, \mathrm{I}, \mathrm{V}, \mathrm{F}, \mathrm{M}, \mathrm{W}$, and C) (Bogerd et al., 1996; Kim et al., 1996a; Zhang and Dayton, 1998). Both of the putative NESs which can theoretically confer CRM1-mediated export are located outside of the sequence region that is missing in the short splicing isoform NF-YCb (amino acid residues 36-97). Hence, if one of these two putative export signals was functional also the over-expressed short splicing isoform NF-YCb would have been localized in the cytoplasm. This, however, was not observed by Frontini et al. (Frontini et al., 2004), making it very unlikely that the cytoplasmic localization of strongly overexpressed NF-YC is caused by nuclear export.

If nuclear import of NF-YB and NF-YC entirely depends on dimer formation and subsequent nuclear translocation mediated by importin 13, one would expect that both HFM containing 
subunits behave analogously if strongly over-expressed. However, strong over-expression of NF-YB did not as dramatically alter nuclear localization as observed for NF-YC (Fig.4B). How can that be explained? Since the monomeric NF-YB subunit was neither bound to importin 13 nor imported into the nucleus of permeabilized cells it is rather unlikely that NF-YB contains an independent NLS which is exposed by NF-YC and enhances binding of importin 13 to NF-YB. Therefore, we assume that NF-YB in contrast to NF-YC is able to interact (in the cytoplasm) with another nuclear protein and then is piggyback transported into the nucleus. Because of the strong over-expression, the potential interacting protein has to be abundant as well. Thus, good candidates for such a scenario could be core histones. During the S-phase of the cell a vast amount core histones have to be imported into the nucleus. In vitro binding studies showed that YBL1 directly interacts with H3 whereas YCL1 does not interact with any of the core histones (Bolognese et al., 2000). Further it was demonstrated that immobilized NF-YB (more specifically the HFM of NF-YB) binds to H3-H4 (Caretti et al., 1999). Taken together, while H2A-like proteins such as YCL1 and NF-YC are incapable of interacting directly with core histones, H2B-like proteins such as YBL1 and NF-YB are able to interact with $\mathrm{H} 3$ or H4, respectively (Bolognese et al., 2000; Caretti et al., 1999). Thus, piggyback transport of NF-YB into the nucleus could be achieved via the ncNLSs in the amino-terminal tails of the core histones H3/H4. In a situation of high abundance as caused by strong over-expression, nuclear localization of NF-YB might therefore not change as dramatically as it does for NF-YC because of binding to $\mathrm{H} 3 / \mathrm{H} 4$ and subsequent piggy-back transport into the nucleus. Whether or not this actually occurs, especially in vivo under physiological conditions, we don't know.

Further, it was shown that the HFM containing subunits NF-YB and NF-YC can obviously act independently from NF-YA and fulfill functions in the absence of the regulatory subunit NF-YA. For instance, NF-YB/NF-YC dimers can efficiently associate with DNA during nucleosome formation and thereby have an intrinsic affinity for H3-H4 (Caretti et al., 1999). These associations, however, are not incompatible with (subsequent) binding of NF-YA and suggest that NF-Y can intervene at different steps during nucleosome formation. In addition, activation assays with HFM containing subunits NF-YB and NF-YC fused to GAL4 also indicated that they are sufficient to activate transcription robustly, two- to fourfold better than the NF-Y trimer (de Silvio et al., 1999), and biochemical evidence suggests that NF-YB and NF-YC are associated with different complexes of high molecular weight in the absence of NF-YA (Bellorini et al., 1997). Moreover, NF-YB and NF-YC, both separately and heterodimerized, bind to the TATA-Box binding protein (TBP) in vitro, and in line with that an association of NF-YB/NF-YC with holo-TFIID was observed (Bellorini et al., 1997). 
The NC2 (NC2 $\alpha / \mathrm{NC} 2 \beta)$ heterodimer (Inostroza et al., 1992; Meisterernst and Roeder, 1991) blocks preinitiation complex (PIC) assembly (inhibition of TATA-dependent transcription) via direct interaction with the TBP-DNA binary complex inhibiting incorporation of TFIIB and TFIIA (Goppelt et al., 1996; Kamada et al., 2001; Kim et al., 1996c). Therefore, an additional function of the NF-YB/NF-YC dimer in the absence of NF-YA could include direct competition with NC2 for the TBP-DNA binary complex. In this case, transcriptional activation and repression would be balanced by the intra-nuclear concentration of NF-YB/NF-YC and $\mathrm{NC} 2 \alpha / \mathrm{NC} 2 \beta$. Whether these two closely related HFM dimers, despite their functional divergence, use the same import mechanism to translocate through the nuclear pore complex (via importin 13), remains to be investigated.

Our findings that the HFM containing subunits NF-YB and NF-YC were imported into the nucleus as a heterodimeric complex by a pathway distinct from NF-YA are completely in line with the observation that the HFM containing subunits can fulfill different nuclear functions independent of NF-YA. Regulated traffic of cargoes between nucleus and cytoplasm is rather the rule than the exception and the efficiency of nuclear transport of a protein depends on its interaction with the components of the transport machinery. In case of the NF-Y subunits this is obviously controlled by complex formation of NF-YB and NF-YC. Transport of the dimerized complex mediated by importin 13 ensures proper nuclear localization of equimolar concentrations of NF-YB and NF-YC.

\subsection{Outlook}

Transcription is the key step at which gene activity is controlled and regulated. Effectors of transcription include RNA polymerases, basal transcription factors (TBP and TAFs), sequencespecific DNA-binding factors that interact with promoters and enhancers (such as NF-Y), chromatin remodeling factors, transcriptional mediators that promote interactions between enhancer-binding factors and the basal transcriptional machinery, and last but not least enzymes catalyzing acetylation, deacetylation, phosphorylation, ubiquitinylation, and methylation of nuclear proteins (reviewed in Burley and Roeder, 1996; Lemon and Tjian, 2000; Orphanides et al., 1996; Orphanides and Reinberg, 2002; Strahl and Allis, 2000). Since active nuclear transport of transcriptional effectors resembles one of the main regulatory processes during signal transduction cascades, further studies should continue to characterize nuclear transport pathways of transcriptional effectors. 
Regarding the preinitiation complex (PIC) assembly it was demonstrated that Kap114p is the transport receptor of TBP (Morehouse et al., 1999; Pemberton et al., 1999) while nuclear import of TFIIA is mediated by Kap122p (Titov and Blobel, 1999) in yeast. However, nuclear import of these two essential proteins mediated by non-essential transport receptors implied that alternative import pathways must exist, which later was confirmed by the interaction of TBP with three other importins (Pemberton et al., 1999). The question whether multiple import pathways exist also for the NF-Y subunits could be addressed as follows: In vitro transcribed and translated, ${ }^{35}$ S-labeled NF-Y subunits have to be co-injected with non-labeled substrates specific for either importin $\beta$ (such as HIV-1 Rev or importin $\alpha /$ IBB domain to characterize uptake of NF-YA) or importin 13 (such as Pax6 or hUBC9 to elucidate the import of the NF-YB/NF-YC dimer) into the cytoplasm of Xenopus oocytes. In both cases, co-injection should reduce the nuclear uptake of the microinjected NF-Y subunits unless redundant nuclear import pathways exist.

With regard to the ncNLS of NF-YA a more comprehensive mutagenesis strategy coupled with quantitative analysis would provide essential information about which amino acid residues besides the characterized positively charged amino acid residues are necessary (and sufficient) to confer importin $\beta$ binding and subsequent nuclear translocation.

To identify the binding sites in importin $\beta$ for NF-YA and in importin 13 for the NF-YB/NF-YC dimer, in vitro binding assays should be performed with deletion constructs of the respective importins (as done for instance by Jakel and Gorlich who mapped the binding site in importin $\beta$ for the BIB domain (Jakel and Gorlich, 1998), or by Bauerle et al. who characterized the importin $\beta$ binding site in importin 7 (Bauerle et al., 2002).

Further, the results of in vitro nuclear import assays clearly demonstrated that nuclear uptake of NF-YA was competed by core histones indicating that at least one of the transport receptors that mediate nuclear import of the four different core histones (importin $\beta$, importin 5 , importin 7 , transportin, and importin 9) was also required for nuclear accumulation of NF-YA. In this work all importins except importin 9 were used to reconstitute nuclear import of NF-YA, therefore further studies should also be directed towards illuminating the possible role of importin 9 for the nuclear transport of NF-YA. 
It might also be interesting to address the question whether importin $\beta$ and importin 13 also play a role in the intranuclear targeting of the NF-Y subunits. For instance, it was demonstrated that double stranded, TATA-containing DNA was necessary to stimulate dissociation of the TATAbox binding protein (TBP) from its transport receptor (Kap114) at lower RanGTP concentrations (Pemberton et al., 1999). Perhaps a similar mechanism does exist for the preferential release of NF-Y subunits from their import receptors at the promoter of genes to be transcribed?

Finally, the histone fold pair of NF-Y (NF-YB-NF-YC) is closest related to the TBP-binding repressor $\mathrm{NC} 2(\mathrm{NC} 2 \alpha-\mathrm{NC} 2 \beta)$ which raises the question whether $\mathrm{NC} 2 \alpha-\mathrm{NC} 2 \beta$ can be recognized by importin 13 . Further studies should definitely address this question. 


\section{Summary}

The CCAAT-specific transcription factor NF-Y is a heterotrimeric complex composed of three subunits, NF-YA, NF-YB, and NF-YC. Each subunit contains a core region that has been highly conserved throughout evolution. Here, we report that in higher eukaryotes the subunits of NF-Y are imported into the nucleus by distinct, signal-dependent, energy-dependent, receptor-mediated pathways. Importin $\beta$ mediates nuclear import of NF-YA whereas heterodimerization of the two histone fold motif (HFM) containing subunits NF-YB and NF-YC is a prerequisite for their nuclear accumulation conferred by importin 13. To our knowledge, the NF-YB/NF-YC dimer represents the first example of an importin 13 specific substrate the recognition of which solely depends on complex formation. The monomeric HFM containing subunits NF-YB and NF-YC lack a functional NLS but the minimal sequence regions necessary for nuclear localization basically correspond to their HFMs. This indicates that in the NF-YB/NF-YC complex a distinct binding platform derived from the HFM of both subunits, NF-YB and NF-YC, mediates the interactions with importin 13. Indeed, this assumption was supported by the fact that binding of importin 13 to NF-YB/NF-YC was competed by NF-YA which also only binds to the dimerized HFM. Thus, the binding sites in the NF-YB/NF-YC dimer for importin 13 and NF-YA overlap, whereas NF-YA has a higher affinity than importin 13 towards the histone fold dimer. In contrast to the NF-YB/NF-YC complex, the H2A/H2B dimer did not behave as an importin 13specific import substrate suggesting that importin 13 does not bind to histone fold pairs in general. NF-YA contains a nonclassical NLS (ncNLS) in the 56 amino acid long evolutionarily conserved region at the $\mathrm{C}$-terminus of the protein, and mutational analysis indicates that a certain number of positively charged amino acid residues in the NLS sequence of NF-YA is required for nuclear accumulation. Importin $\beta$ binding is restricted to the monomeric, uncomplexed NF-YA subunit. Thus NF-YA can not mediate the interaction of importin $\beta$ with the functional NF-Y holocomplex.

Redundant receptor-mediated and energy-dependent pathways exist for the nuclear import of core histones. While specific importins serve as primary receptors in yeast, at least five members of the importin $\beta$ superfamily (importin $\beta$, importin 5 , importin 7 , importin 9 , and transportin) mediate nuclear import of core histones in mammals (in vitro). Regarding their structure the core histones show an overall similarity, consisting of unstructured, basic amino- and carboxyterminal domains and hydrophobic, central globular domains that form histone fold motifs. Previously, in vivo transfection experiments demonstrated that both the amino-terminal tails and the central globular domains of all core histones can serve as regions involved in nuclear 
targeting. However, here we report that import receptors only interact directly with the aminoterminal tails of core histones (in vitro binding and nuclear import assays). The result that globular histone domains (containing a HFM) are not recognized by importins is in line with the data obtained from the HFM containing subunits NF-YB and NF-YC and strongly suggests that the HFM represents a protein-protein interaction domain but not a NLS per se. Hence, the nuclear transport competence of globular domains of core histones in vivo depends most likely on the formation of histone heterodimers (oligomers). The results of in vitro nuclear import assays showed that the globular domain of H2B can be piggyback transported into the nucleus via the ncNLS in the amino-terminal tail of the corresponding partner histone H2A. These results further indicate that histone import signals are still accessible to importin binding when $\mathrm{H} 2 \mathrm{~A}$ and H2B are dimerized. Further, preliminary data demonstrated that binding of importin $\beta$ to H2B can be competed by addition of either BIB or IBB domain suggesting that core histones (aminoterminal tails) bind to the IBB-like cargo binding site I on importin $\beta$. 


\section{Zusammenfassung}

Der CCAAT-Box spezifische Transkriptionsfaktor NF-Y ist ein trimerer Komplex, der aus den drei Untereinheiten NF-YA, NF-YB und NF-YC besteht. Jede dieser Untereinheiten besitzt einen evolutionär konservierten Bereich, der sowohl für die Bildung des trimeren Komplexes als auch für die DNA-Bindung wichtig ist. Die konservierten Bereiche von NF-YB und NF-YC enthalten das so genannte "histone-fold-motif" (HFM) als wesentliches Strukturelement und die beiden Proteine sind Mitglieder der H2A/H2B-Familie. In dieser Arbeit konnte gezeigt werden, dass die Untereinheiten von NF-Y in höheren Eukaryoten auf verschiedenen, signalabhängigen, energieabhängigen, rezeptorvermittelten Wegen in den Zellkern importiert werden. Importin $\beta$ vermittelt den Kerntransport von NF-YA. Die Dimerisierung der beiden HFM-Untereinheiten NF-YB und NF-YC ist dagegen die Voraussetzung für einen über Importin13 vermittelten Kerntransport. Nach unserer Kenntnis ist der NF-YB/NF-YC-Dimer das erste Beispiel für ein Importin13-Substrat, dessen Kerntransport ausschließlich von der Komplexbildung abhängt. Die NF-YB- und NF-YC-Monomere besitzen keine funktionellen Kernlokalisierungssignale (NLS), allerdings stimmen die für eine Kernlokalisierung (des Dimers) notwendigen minimalen Sequenzabschnitte mit dem jeweiligen HFM überein. Dies deutet darauf hin, dass die Basis für die Interaktion mit Importin13 durch eine Dimerisierung der HFM beider Untereinheiten geschaffen wird. Diese Annahme wurde dadurch bekräftigt, dass NF-YA mit Importin13 um die Bindung an den NF-YB/NF-YC-Komplex konkurriert. Dabei ist bekannt, dass NF-YA ebenfalls nur an die dimerisierten Untereinheiten bindet. Daraus ist zu schließen, dass die Bindungsstellen für Importin13 und NF-YA überlappen, wobei NF-YA mit einer höheren Affinität an den NF-YB/NF-YC-Dimer bindet als Importin13. Im Gegensatz zu dem NF-YB/NF-YC-Komplex erwies sich der H2A/H2B-Dimer nicht als Importin13-Substrat. Dies lässt vermuten, dass Importin13 nicht grundsätzlich HFM-Paare erkennt und transportiert. Im Fall von NF-YA wurde ein NLS in dem (56 Aminosäuren langen) evolutionär konservierten Bereich am C-Terminus des Proteins identifiziert. Mutationen verschiedener Codons für basische Aminosäurereste innerhalb dieses NLS führten zu einer deutlichen Verminderung der Kernlokalisierung von NF-YA. Dieses Ergebnis legt die Vermutung nahe, dass eine gewisse Anzahl an basischen Aminosäuren innerhalb des NLS für dessen Funktion nötig ist. Die Bindung von Importin $\beta$ an NF-YA ist auf die monomere Untereinheit beschränkt. Eine Interaktion zwischen Importin $\beta$ und dem funktionellen NF-Y-Komplex über NF-YA kann vermutlich nicht geschehen, da Bereiche des NLS in NF-YA ebenfalls zur Bindung an den NF-YB/NF-YC-Dimer benötigt werden. 
Der Kerntransport der Core-Histone erfolgt über redundante, rezeptorvermittelte und energieabhängige Transportwege. Während in Hefe spezielle Importfaktoren als primäre Rezeptoren fungieren, können in Säugetieren wenigstens fünf Mitglieder der Importin $\beta$-Familie (Importin $\beta$, Importin7, Importin5, Importin9 und Transportin) den Transport der Core-Histone in den Zellkern vermitteln. Die verschiedenen Core-Histone weisen eine allgemeine strukturelle Ähnlichkeit auf. Sie bestehen aus unstrukturierten, basischen Amino- und Carboxy-terminalen Abschnitten sowie einer hydrophoben, zentralen globulären Domäne, dessen Grundelement das HFM ist. Frühere in vivo-Transfektionsexperimente in HeLa-Zellen haben offenbart, dass sowohl die Amino-terminalen Bereiche als auch die globulären Domänen der Core-Histone in der Lage sind, Fusionsproteine in den Kern zu dirigieren. In dieser Arbeit konnten wir allerdings durch in vitro-Bindungsstudien und in vitro-Import-Experimente zeigen, dass die Importfaktoren nur mit den Amino-terminalen Bereichen der Core-Histone interagieren. Die HFM-enthaltenden globulären Domänen der Core-Histone werden wie die HFM-Untereinheiten NF-YB und NF-YC nicht von Tranportfaktoren erkannt. Dies lässt darauf schließen, dass das HFM ein ProteinProtein-Interaktionsmotiv darstellt, aber kein NLS per se. Die in den in vivo-Transfektionsexperimenten gezeigte Transport-Kompetenz der globulären Domänen der Core-Histone beruht demzufolge wahrscheinlich auf der Bildung von Histon-Dimeren (oder -Oligomeren). Die Ergebnisse unserer in vitro-Import-Experimente zeigen, dass die globuläre Domäne von H2B über das NLS im Amino-terminalen Bereich des entsprechenden Partner-Histons H2A in den Zellkern transportiert werden kann. Die Ergebnisse verdeutlichen ferner, dass die Importsignale der Core-Histone auch noch nach Dimerisierung von H2A und H2B für die Importfaktoren zugänglich sind. Darüber hinaus konnte in ersten Experimenten gezeigt werden, dass die Bindung von Importin $\beta$ an H2B durch die Zugabe der BIB- oder der IBB-Domäne reduziert wird. Dies lässt darauf schließen, dass die in den Amino-terminalen Bereichen enthaltenen NLS der Core-Histone an die Bindungsstelle I (IBB-ähnliche Bindungsstelle) in Importin $\beta$ binden. 


\section{References}

Adam, S.A., Marr, R.S. and Gerace, L. (1990) Nuclear protein import in permeabilized mammalian cells requires soluble cytoplasmic factors. J Cell Biol, 111, 807-816.

Adams, C.R. and Kamakaka, R.T. (1999) Chromatin assembly: biochemical identities and genetic redundancy. Curr Opin Genet Dev, 9, 185-190.

Akey, C.W. and Radermacher, M. (1993) Architecture of the Xenopus nuclear pore complex revealed by three-dimensional cryo-electron microscopy. J Cell Biol, 122, 1-19.

Allan, J., Mitchell, T., Harborne, N., Bohm, L. and Crane-Robinson, C. (1986) Roles of H1 domains in determining higher order chromatin structure and H1 location. J Mol Biol, 187, 591-601.

Andrade, M.A. and Bork, P. (1995) HEAT repeats in the Huntington's disease protein. Nat Genet, 11, 115-116.

Arents, G. and Moudrianakis, E.N. (1995) The histone fold: a ubiquitous architectural motif utilized in DNA compaction and protein dimerization. Proc Natl Acad Sci U S A, 92, 11170-11174.

Arts, G.J., Fornerod, M. and Mattaj, I.W. (1998) Identification of a nuclear export receptor for tRNA. Curr Biol, 8, 305-314.

Baake, M., Bauerle, M., Doenecke, D. and Albig, W. (2001a) Core histones and linker histones are imported into the nucleus by different pathways. Eur J Cell Biol, 80, 669-677.

Baake, M., Doenecke, D. and Albig, W. (2001b) Characterisation of nuclear localisation signals of the four human core histones. J Cell Biochem, 81, 333-346.

Bauerle, M., Doenecke, D. and Albig, W. (2002) The requirement of H1 histones for a heterodimeric nuclear import receptor. J Biol Chem, 277, 32480-32489.

Baxevanis, A.D., Arents, G., Moudrianakis, E.N. and Landsman, D. (1995) A variety of DNA-binding and multimeric proteins contain the histone fold motif. Nucleic Acids Res, 23, 2685-2691.

Bayliss, R., Littlewood, T. and Stewart, M. (2000) Structural basis for the interaction between FxFG nucleoporin repeats and importin-beta in nuclear trafficking. Cell, 102, 99-108.

Bellorini, M., Lee, D.K., Dantonel, J.C., Zemzoumi, K., Roeder, R.G., Tora, L. and Mantovani, R. (1997) CCAAT binding NF-Y-TBP interactions: NF-YB and NF-YC require short domains adjacent to their histone fold motifs for association with TBP basic residues. Nucleic Acids Res, 25, 21742181.

Berry, M., Grosveld, F. and Dillon, N. (1992) A single point mutation is the cause of the Greek form of hereditary persistence of fetal haemoglobin. Nature, 358, 499-502.

Bhattacharya, A., Deng, J.M., Zhang, Z., Behringer, R., de Crombrugghe, B. and Maity, S.N. (2003) The $\mathrm{B}$ subunit of the CCAAT box binding transcription factor complex (CBF/NF-Y) is essential for early mouse development and cell proliferation. Cancer Res, 63, 8167-8172.

Bi, W., Wu, L., Coustry, F., de Crombrugghe, B. and Maity, S.N. (1997) DNA binding specificity of the CCAAT-binding factor CBF/NF-Y. J Biol Chem, 272, 26562-26572. 
Bischoff, F.R. and Gorlich, D. (1997) RanBP1 is crucial for the release of RanGTP from importin betarelated nuclear transport factors. FEBS Lett, 419, 249-254.

Bischoff, F.R., Scheffzek, K. and Ponstingl, H. (2002) How Ran is regulated. Results Probl Cell Differ, 35, 49-66.

Bogerd, H.P., Benson, R.E., Truant, R., Herold, A., Phingbodhipakkiya, M. and Cullen, B.R. (1999) Definition of a consensus transportin-specific nucleocytoplasmic transport signal. J Biol Chem, 274, 9771-9777.

Bogerd, H.P., Fridell, R.A., Benson, R.E., Hua, J. and Cullen, B.R. (1996) Protein sequence requirements for function of the human T-cell leukemia virus type 1 Rex nuclear export signal delineated by a novel in vivo randomization-selection assay. Mol Cell Biol, 16, 4207-4214.

Bohnsack, M.T., Regener, K., Schwappach, B., Saffrich, R., Paraskeva, E., Hartmann, E. and Gorlich, D. (2002) Exp5 exports eEF1A via tRNA from nuclei and synergizes with other transport pathways to confine translation to the cytoplasm. EMBO J, 21, 6205-6215.

Bolognese, F., Imbriano, C., Caretti, G. and Mantovani, R. (2000) Cloning and characterization of the histone-fold proteins YBL1 and YCL1. Nucleic Acids Res, 28, 3830-3838.

Bradford, M.M. (1976) A rapid and sensitive method for the quantitation of microgram quantities of protein utilizing the principle of protein-dye binding. Anal Biochem, 72, 248-254.

Braun, I.C., Herold, A., Rode, M., Conti, E. and Izaurralde, E. (2001) Overexpression of TAP/p15 heterodimers bypasses nuclear retention and stimulates nuclear mRNA export. J Biol Chem, 276, 20536-20543.

Braun, I.C., Herold, A., Rode, M. and Izaurralde, E. (2002) Nuclear export of mRNA by TAP/NXF1 requires two nucleoporin-binding sites but not p15. Mol Cell Biol, 22, 5405-5418.

Breeuwer, M. and Goldfarb, D.S. (1990) Facilitated nuclear transport of histone H1 and other small nucleophilic proteins. Cell, 60, 999-1008.

Bucher, P. (1990) Weight matrix descriptions of four eukaryotic RNA polymerase II promoter elements derived from 502 unrelated promoter sequences. J Mol Biol, 212, 563-578.

Burley, S.K. and Roeder, R.G. (1996) Biochemistry and structural biology of transcription factor IID (TFIID). Annu Rev Biochem, 65, 769-799.

Calado, A., Treichel, N., Muller, E.C., Otto, A. and Kutay, U. (2002) Exportin-5-mediated nuclear export of eukaryotic elongation factor 1A and tRNA. EMBO J, 21, 6216-6224.

Caretti, G., Motta, M.C. and Mantovani, R. (1999) NF-Y associates with H3-H4 tetramers and octamers by multiple mechanisms. Mol Cell Biol, 19, 8591-8603.

Caretti, G., Salsi, V., Vecchi, C., Imbriano, C. and Mantovani, R. (2003) Dynamic recruitment of NF-Y and histone acetyltransferases on cell-cycle promoters. J Biol Chem, 278, 30435-30440.

Chen, F., Ogawa, K., Liu, X., Stringfield, T.M. and Chen, Y. (2002) Repression of Smad2 and Smad3 transactivating activity by association with a novel splice variant of CCAAT-binding factor $\mathrm{C}$ subunit. Biochem J, 364, 571-577. 
Chook, Y.M. and Blobel, G. (2001) Karyopherins and nuclear import. Curr Opin Struct Biol, 11, 703715 .

Christophe, D., Christophe-Hobertus, C. and Pichon, B. (2000) Nuclear targeting of proteins: how many different signals? Cell Signal, 12, 337-341.

Cingolani, G., Bednenko, J., Gillespie, M.T. and Gerace, L. (2002) Molecular basis for the recognition of a nonclassical nuclear localization signal by importin beta. Mol Cell, 10, 1345-1353.

Cingolani, G., Lashuel, H.A., Gerace, L. and Muller, C.W. (2000) Nuclear import factors importin alpha and importin beta undergo mutually induced conformational changes upon association. FEBS Lett, 484, 291-298.

Cingolani, G., Petosa, C., Weis, K. and Muller, C.W. (1999) Structure of importin-beta bound to the IBB domain of importin-alpha. Nature, 399, 221-229.

Cokol, M., Nair, R. and Rost, B. (2000) Finding nuclear localization signals. EMBO Rep, 1, 411-415.

Conti, E. (2002) Structures of importins. Results Probl Cell Differ, 35, 93-113.

Conti, E. and Izaurralde, E. (2001) Nucleocytoplasmic transport enters the atomic age. Curr Opin Cell Biol, 13, 310-319.

Conti, E., Uy, M., Leighton, L., Blobel, G. and Kuriyan, J. (1998) Crystallographic analysis of the recognition of a nuclear localization signal by the nuclear import factor karyopherin alpha. Cell, 94, 193-204.

Cronshaw, J.M., Krutchinsky, A.N., Zhang, W., Chait, B.T. and Matunis, M.J. (2002) Proteomic analysis of the mammalian nuclear pore complex. J Cell Biol, 158, 915-927.

Davis, B.J. (1964) Disc Electrophoresis. Ii. Method and Application to Human Serum Proteins. Ann N Y Acad Sci, 121, 404-427.

de Silvio, A., Imbriano, C. and Mantovani, R. (1999) Dissection of the NF-Y transcriptional activation potential. Nucleic Acids Res, 27, 2578-2584.

Dingwall, C. and Laskey, R.A. (1991) Nuclear targeting sequences--a consensus? Trends Biochem Sci, 16, 478-481.

Dorn, A., Bollekens, J., Staub, A., Benoist, C. and Mathis, D. (1987) A multiplicity of CCAAT boxbinding proteins. Cell, $\mathbf{5 0}, 863-872$.

Dumont, J.N. (1972) Oogenesis in Xenopus laevis (Daudin). I. Stages of oocyte development in laboratory maintained animals. J Morphol, 136, 153-179.

Earnshaw, W.C., Honda, B.M., Laskey, R.A. and Thomas, J.O. (1980) Assembly of nucleosomes: the reaction involving X. laevis nucleoplasmin. Cell, 21, 373-383.

Englmeier, L., Olivo, J.C. and Mattaj, I.W. (1999) Receptor-mediated substrate translocation through the nuclear pore complex without nucleotide triphosphate hydrolysis. Curr Biol, 9, 30-41.

Fahrenkrog, B. and Aebi, U. (2003) The nuclear pore complex: nucleocytoplasmic transport and beyond. Nat Rev Mol Cell Biol, 4, 757-766. 
Fahrenkrog, B., Koser, J. and Aebi, U. (2004) The nuclear pore complex: a jack of all trades? Trends Biochem Sci, 29, 175-182.

Fahrenkrog, B., Stoffler, D. and Aebi, U. (2001) Nuclear pore complex architecture and functional dynamics. Curr Top Microbiol Immunol, 259, 95-117.

Farabaugh, P.J. (1978) Sequence of the lacI gene. Nature, 274, 765-769.

Finlay, D.R., Newmeyer, D.D., Price, T.M. and Forbes, D.J. (1987) Inhibition of in vitro nuclear transport by a lectin that binds to nuclear pores. $J$ Cell Biol, 104, 189-200.

Fischer, U., Huber, J., Boelens, W.C., Mattaj, I.W. and Luhrmann, R. (1995) The HIV-1 Rev activation domain is a nuclear export signal that accesses an export pathway used by specific cellular RNAs. Cell, 82, 475-483.

Fornerod, M. and Ohno, M. (2002) Exportin-mediated nuclear export of proteins and ribonucleoproteins. Results Probl Cell Differ, 35, 67-91.

Fornerod, M., Ohno, M., Yoshida, M. and Mattaj, I.W. (1997a) CRM1 is an export receptor for leucinerich nuclear export signals. Cell, 90, 1051-1060.

Fornerod, M., van Deursen, J., van Baal, S., Reynolds, A., Davis, D., Murti, K.G., Fransen, J. and Grosveld, G. (1997b) The human homologue of yeast CRM1 is in a dynamic subcomplex with CAN/Nup214 and a novel nuclear pore component Nup88. EMBO J, 16, 807-816.

Forwood, J.K., Lam, M.H. and Jans, D.A. (2001) Nuclear import of Creb and AP-1 transcription factors requires importin-beta 1 and Ran but is independent of importin-alpha. Biochemistry, 40, 52085217.

Freeman, L., Kurumizaka, H. and Wolffe, A.P. (1996) Functional domains for assembly of histones H3 and H4 into the chromatin of Xenopus embryos. Proc Natl Acad Sci U S A, 93, 12780-12785.

Fried, H. and Kutay, U. (2003) Nucleocytoplasmic transport: taking an inventory. Cell Mol Life Sci, 60, 1659-1688.

Frontini, M., Imbriano, C., Manni, I. and Mantovani, R. (2004) Cell cycle regulation of NF-YC nuclear localization. Cell Cycle, 3, 217-222.

Fukuda, M., Asano, S., Nakamura, T., Adachi, M., Yoshida, M., Yanagida, M. and Nishida, E. (1997) CRM1 is responsible for intracellular transport mediated by the nuclear export signal. Nature, 390, 308-311.

Goppelt, A., Stelzer, G., Lottspeich, F. and Meisterernst, M. (1996) A mechanism for repression of class II gene transcription through specific binding of NC2 to TBP-promoter complexes via heterodimeric histone fold domains. EMBO J, 15, 3105-3116.

Gorlich, D., Dabrowski, M., Bischoff, F.R., Kutay, U., Bork, P., Hartmann, E., Prehn, S. and Izaurralde, E. (1997) A novel class of RanGTP binding proteins. J Cell Biol, 138, 65-80.

Gorlich, D., Henklein, P., Laskey, R.A. and Hartmann, E. (1996a) A 41 amino acid motif in importinalpha confers binding to importin-beta and hence transit into the nucleus. $E M B O J, \mathbf{1 5}, 1810$ 1817. 
Gorlich, D. and Kutay, U. (1999) Transport between the cell nucleus and the cytoplasm. Annu Rev Cell Dev Biol, 15, 607-660.

Gorlich, D. and Mattaj, I.W. (1996) Nucleocytoplasmic transport. Science, 271, 1513-1518.

Gorlich, D., Pante, N., Kutay, U., Aebi, U. and Bischoff, F.R. (1996b) Identification of different roles for RanGDP and RanGTP in nuclear protein import. EMBO J, 15, 5584-5594.

Gorlich, D., Prehn, S., Laskey, R.A. and Hartmann, E. (1994) Isolation of a protein that is essential for the first step of nuclear protein import. Cell, 79, 767-778.

Gorlich, D., Seewald, M.J. and Ribbeck, K. (2003) Characterization of Ran-driven cargo transport and the RanGTPase system by kinetic measurements and computer simulation. EMBO J, 22, 1088-1100.

Gorlich, D., Vogel, F., Mills, A.D., Hartmann, E. and Laskey, R.A. (1995) Distinct functions for the two importin subunits in nuclear protein import. Nature, 377, 246-248.

Greiner, M., Caesar, S. and Schlenstedt, G. (2004) The histones H2A/H2B and H3/H4 are imported into the yeast nucleus by different mechanisms. Eur J Cell Biol, 83, 511-520.

Gurdon, J.B. (1977) Methods for nuclear transplantation in amphibia. Methods Cell Biol, 16, 125-139.

Harel, A. and Forbes, D.J. (2004) Importin beta: conducting a much larger cellular symphony. Mol Cell, 16, 319-330.

Henderson, B.R. and Eleftheriou, A. (2000) A comparison of the activity, sequence specificity, and CRM1-dependence of different nuclear export signals. Exp Cell Res, 256, 213-224.

Henderson, B.R. and Percipalle, P. (1997) Interactions between HIV Rev and nuclear import and export factors: the Rev nuclear localisation signal mediates specific binding to human importin-beta. $J$ Mol Biol, 274, 693-707.

Hernandez, N. (1993) TBP, a universal eukaryotic transcription factor? Genes Dev, 7, 1291-1308.

Hinshaw, J.E., Carragher, B.O. and Milligan, R.A. (1992) Architecture and design of the nuclear pore complex. Cell, 69, 1133-1141.

Ho, S.N., Hunt, H.D., Horton, R.M., Pullen, J.K. and Pease, L.R. (1989) Site-directed mutagenesis by overlap extension using the polymerase chain reaction. Gene, 77, 51-59.

Hochuli, E., Dobeli, H. and Schacher, A. (1987) New metal chelate adsorbent selective for proteins and peptides containing neighbouring histidine residues. J Chromatogr, 411, 177-184.

Imamoto, N. (2000) Diversity in nucleocytoplasmic transport pathways. Cell Struct Funct, 25, 207-216.

Inostroza, J.A., Mermelstein, F.H., Ha, I., Lane, W.S. and Reinberg, D. (1992) Dr1, a TATA-binding protein-associated phosphoprotein and inhibitor of class II gene transcription. Cell, 70, 477-489.

Ito, T., Bulger, M., Kobayashi, R. and Kadonaga, J.T. (1996) Drosophila NAP-1 is a core histone chaperone that functions in ATP-facilitated assembly of regularly spaced nucleosomal arrays. Mol Cell Biol, 16, 3112-3124.

Ito, T., Tyler, J.K. and Kadonaga, J.T. (1997) Chromatin assembly factors: a dual function in nucleosome formation and mobilization? Genes Cells, 2, 593-600.

Izaurralde, E. (2002) Nuclear export of messenger RNA. Results Probl Cell Differ, 35, 133-150. 
Izaurralde, E., Kutay, U., von Kobbe, C., Mattaj, I.W. and Gorlich, D. (1997) The asymmetric distribution of the constituents of the Ran system is essential for transport into and out of the nucleus. EMBO $J, \mathbf{1 6}, 6535-6547$.

Jakel, S., Albig, W., Kutay, U., Bischoff, F.R., Schwamborn, K., Doenecke, D. and Gorlich, D. (1999) The importin beta/importin 7 heterodimer is a functional nuclear import receptor for histone H1. EMBO J, 18, 2411-2423.

Jakel, S. and Gorlich, D. (1998) Importin beta, transportin, RanBP5 and RanBP7 mediate nuclear import of ribosomal proteins in mammalian cells. EMBO J, 17, 4491-4502.

Jakel, S., Mingot, J.M., Schwarzmaier, P., Hartmann, E. and Gorlich, D. (2002) Importins fulfil a dual function as nuclear import receptors and cytoplasmic chaperones for exposed basic domains. EMBO J, 21, 377-386.

Jans, D.A., Xiao, C.Y. and Lam, M.H. (2000) Nuclear targeting signal recognition: a key control point in nuclear transport? Bioessays, 22, 532-544.

Johnson, A.W., Lund, E. and Dahlberg, J. (2002) Nuclear export of ribosomal subunits. Trends Biochem Sci, 27, 580-585.

Kaffman, A. and O'Shea, E.K. (1999) Regulation of nuclear localization: a key to a door. Annu Rev Cell Dev Biol, 15, 291-339.

Kaffman, A., Rank, N.M., O'Neill, E.M., Huang, L.S. and O'Shea, E.K. (1998a) The receptor Msn5 exports the phosphorylated transcription factor Pho4 out of the nucleus. Nature, 396, 482-486.

Kaffman, A., Rank, N.M. and O'Shea, E.K. (1998b) Phosphorylation regulates association of the transcription factor Pho4 with its import receptor Pse1/Kap121. Genes Dev, 12, 2673-2683.

Kalab, P., Weis, K. and Heald, R. (2002) Visualization of a Ran-GTP gradient in interphase and mitotic Xenopus egg extracts. Science, 295, 2452-2456.

Kalderon, D., Roberts, B.L., Richardson, W.D. and Smith, A.E. (1984) A short amino acid sequence able to specify nuclear location. Cell, 39, 499-509.

Kamada, K., Shu, F., Chen, H., Malik, S., Stelzer, G., Roeder, R.G., Meisterernst, M. and Burley, S.K. (2001) Crystal structure of negative cofactor 2 recognizing the TBP-DNA transcription complex. Cell, 106, 71-81.

Kent, H.M., Clarkson, W.D., Bullock, T.L. and Stewart, M. (1996) Crystallization and preliminary X-ray diffraction analysis of nuclear transport factor 2. J Struct Biol, 116, 326-329.

Kim, C.G. and Sheffery, M. (1990) Physical characterization of the purified CCAAT transcription factor, alpha-CP1. J Biol Chem, 265, 13362-13369.

Kim, F.J., Beeche, A.A., Hunter, J.J., Chin, D.J. and Hope, T.J. (1996a) Characterization of the nuclear export signal of human T-cell lymphotropic virus type 1 Rex reveals that nuclear export is mediated by position-variable hydrophobic interactions. Mol Cell Biol, 16, 5147-5155.

Kim, I.S., Sinha, S., de Crombrugghe, B. and Maity, S.N. (1996b) Determination of functional domains in the $\mathrm{C}$ subunit of the CCAAT-binding factor (CBF) necessary for formation of a CBF-DNA 
complex: CBF-B interacts simultaneously with both the CBF-A and CBF-C subunits to form a heterotrimeric CBF molecule. Mol Cell Biol, 16, 4003-4013.

Kim, J., Parvin, J.D., Shykind, B.M. and Sharp, P.A. (1996c) A negative cofactor containing Dr1/p19 modulates transcription with TFIIA in a promoter-specific fashion. J Biol Chem, 271, 1840518412.

Kim, V.N. (2004) MicroRNA precursors in motion: exportin-5 mediates their nuclear export. Trends Cell Biol, 14, 156-159.

Kobe, B. (1999) Autoinhibition by an internal nuclear localization signal revealed by the crystal structure of mammalian importin alpha. Nat Struct Biol, 6, 388-397.

Koessler, H., Kahle, J., Bode, C., Doenecke, D. and Albig, W. (2004) Human replication dependent histone $\mathrm{H} 3$ genes are activated by a tandemly arranged pair of two CCAAT-boxes. Biochem J, Pt.

Kolodrubetz, D., Rykowski, M.C. and Grunstein, M. (1982) Histone H2A subtypes associate interchangeably in vivo with histone H2B subtypes. Proc Natl Acad Sci U S A, 79, 7814-7818.

Komeili, A. and O'Shea, E.K. (1999) Roles of phosphorylation sites in regulating activity of the transcription factor Pho4. Science, 284, 977-980.

Kornberg, R.D. and Lorch, Y. (1999) Twenty-five years of the nucleosome, fundamental particle of the eukaryote chromosome. Cell, 98, 285-294.

Kose, S., Imamoto, N., Tachibana, T., Shimamoto, T. and Yoneda, Y. (1997) Ran-unassisted nuclear migration of a 97-kD component of nuclear pore-targeting complex. J Cell Biol, 139, 841-849.

Kurz, M., Doenecke, D. and Albig, W. (1997) Nuclear transport of H1 histones meets the criteria of a nuclear localization signal-mediated process. J Cell Biochem, 64, 573-578.

Kutay, U., Bischoff, F.R., Kostka, S., Kraft, R. and Gorlich, D. (1997a) Export of importin alpha from the nucleus is mediated by a specific nuclear transport factor. Cell, 90, 1061-1071.

Kutay, U., Izaurralde, E., Bischoff, F.R., Mattaj, I.W. and Gorlich, D. (1997b) Dominant-negative mutants of importin-beta block multiple pathways of import and export through the nuclear pore complex. EMBO J, 16, 1153-1163.

Kutay, U., Lipowsky, G., Izaurralde, E., Bischoff, F.R., Schwarzmaier, P., Hartmann, E. and Gorlich, D. (1998) Identification of a tRNA-specific nuclear export receptor. Mol Cell, 1, 359-369.

Laemmli, U.K. (1970) Cleavage of structural proteins during the assembly of the head of bacteriophage T4. Nature, 227, 680-685.

Lam, M.H., Briggs, L.J., Hu, W., Martin, T.J., Gillespie, M.T. and Jans, D.A. (1999) Importin beta recognizes parathyroid hormone-related protein with high affinity and mediates its nuclear import in the absence of importin alpha. J Biol Chem, 274, 7391-7398.

Lanford, R.E. and Butel, J.S. (1984) Construction and characterization of an SV40 mutant defective in nuclear transport of T antigen. Cell, 37, 801-813.

Laskey, R.A. and Dingwall, C. (1993) Nuclear shuttling: the default pathway for nuclear proteins? Cell, 74, 585-586. 
Lee, S.J., Imamoto, N., Sakai, H., Nakagawa, A., Kose, S., Koike, M., Yamamoto, M., Kumasaka, T., Yoneda, Y. and Tsukihara, T. (2000) The adoption of a twisted structure of importin-beta is essential for the protein-protein interaction required for nuclear transport. $J$ Mol Biol, 302, 251264.

Lei, E.P. and Silver, P.A. (2002) Protein and RNA export from the nucleus. Dev Cell, 2, 261-272.

Lemon, B. and Tjian, R. (2000) Orchestrated response: a symphony of transcription factors for gene control. Genes Dev, 14, 2551-2569.

Li, Q., Herrler, M., Landsberger, N., Kaludov, N., Ogryzko, V.V., Nakatani, Y. and Wolffe, A.P. (1998) Xenopus NF-Y pre-sets chromatin to potentiate p300 and acetylation-responsive transcription from the Xenopus hsp70 promoter in vivo. EMBO J, 17, 6300-6315.

Lipowsky, G., Bischoff, F.R., Schwarzmaier, P., Kraft, R., Kostka, S., Hartmann, E., Kutay, U. and Gorlich, D. (2000) Exportin 4: a mediator of a novel nuclear export pathway in higher eukaryotes. EMBO J, 19, 4362-4371.

Luger, K., Mader, A.W., Richmond, R.K., Sargent, D.F. and Richmond, T.J. (1997) Crystal structure of the nucleosome core particle at 2.8 A resolution. Nature, 389, 251-260.

Lund, E. and Dahlberg, J.E. (1998) Proofreading and aminoacylation of tRNAs before export from the nucleus. Science, 282, 2082-2085.

Lund, E., Guttinger, S., Calado, A., Dahlberg, J.E. and Kutay, U. (2004) Nuclear export of microRNA precursors. Science, 303, 95-98.

Macara, I.G. (2001) Transport into and out of the nucleus. Microbiol Mol Biol Rev, 65, 570-594, table of contents.

Mahajan, R., Delphin, C., Guan, T., Gerace, L. and Melchior, F. (1997) A small ubiquitin-related polypeptide involved in targeting RanGAP1 to nuclear pore complex protein RanBP2. Cell, 88, 97-107.

Maity, S.N. and de Crombrugghe, B. (1998) Role of the CCAAT-binding protein CBF/NF-Y in transcription. Trends Biochem Sci, 23, 174-178.

Malik, H.S., Eickbush, T.H. and Goldfarb, D.S. (1997) Evolutionary specialization of the nuclear targeting apparatus. Proc Natl Acad Sci U S A, 94, 13738-13742.

Mantovani, R. (1998) A survey of 178 NF-Y binding CCAAT boxes. Nucleic Acids Res, 26, 1135-1143.

Mantovani, R. (1999) The molecular biology of the CCAAT-binding factor NF-Y. Gene, 239, 15-27.

Mantovani, R., Li, X.Y., Pessara, U., Hooft van Huisjduijnen, R., Benoist, C. and Mathis, D. (1994) Dominant negative analogs of NF-YA. J Biol Chem, 269, 20340-20346.

Marshallsay, C., Dickmanns, A., Bischoff, F.R., Ponstingl, H., Fanning, E. and Luhrmann, R. (1996) In vitro and in vivo evidence that protein and U1 snRNP nuclear import in somatic cells differ in their requirement for GTP-hydrolysis, Ran/TC4 and RCC1. Nucleic Acids Res, 24, 1829-1836.

Marziali, G., Perrotti, E., Ilari, R., Testa, U., Coccia, E.M. and Battistini, A. (1997) Transcriptional regulation of the ferritin heavy-chain gene: the activity of the CCAAT binding factor NF-Y is 
modulated in heme-treated Friend leukemia cells and during monocyte-to-macrophage differentiation. Mol Cell Biol, 17, 1387-1395.

Matsuura, Y. and Stewart, M. (2004) Structural basis for the assembly of a nuclear export complex. Nature, 432, 872-877.

Mattaj, I.W. and Conti, E. (1999) Cell biology. Snail mail to the nucleus. Nature, 399, 208-210.

Mattaj, I.W. and Englmeier, L. (1998) Nucleocytoplasmic transport: the soluble phase. Annu Rev Biochem, 67, 265-306.

Matunis, M.J., Coutavas, E. and Blobel, G. (1996) A novel ubiquitin-like modification modulates the partitioning of the Ran-GTPase-activating protein RanGAP1 between the cytosol and the nuclear pore complex. J Cell Biol, 135, 1457-1470.

McBryant, S.J., Park, Y.J., Abernathy, S.M., Laybourn, P.J., Nyborg, J.K. and Luger, K. (2003) Preferential binding of the histone (H3-H4)2 tetramer by NAP1 is mediated by the aminoterminal histone tails. J Biol Chem, 278, 44574-44583.

McNabb, D.S., Xing, Y. and Guarente, L. (1995) Cloning of yeast HAP5: a novel subunit of a heterotrimeric complex required for CCAAT binding. Genes Dev, 9, 47-58.

Meisterernst, M. and Roeder, R.G. (1991) Family of proteins that interact with TFIID and regulate promoter activity. Cell, 67, 557-567.

Melchior, F. and Gerace, L. (1995) Mechanisms of nuclear protein import. Curr Opin Cell Biol, 7, 310318.

Michael, W.M., Choi, M. and Dreyfuss, G. (1995) A nuclear export signal in hnRNP A1: a signalmediated, temperature-dependent nuclear protein export pathway. Cell, 83, 415-422.

Mingot, J.M., Bohnsack, M.T., Jakle, U. and Gorlich, D. (2004) Exportin 7 defines a novel general nuclear export pathway. EMBO J, 23, 3227-3236.

Mingot, J.M., Kostka, S., Kraft, R., Hartmann, E. and Gorlich, D. (2001) Importin 13: a novel mediator of nuclear import and export. EMBO J, 20, 3685-3694.

Moore, M.S. and Blobel, G. (1992) The two steps of nuclear import, targeting to the nuclear envelope and translocation through the nuclear pore, require different cytosolic factors. Cell, 69, 939-950.

Moore, M.S. and Blobel, G. (1993) The GTP-binding protein Ran/TC4 is required for protein import into the nucleus. Nature, 365, 661-663.

Moore, M.S. and Blobel, G. (1994) Purification of a Ran-interacting protein that is required for protein import into the nucleus. Proc Natl Acad Sci U S A, 91, 10212-10216.

Morehouse, H., Buratowski, R.M., Silver, P.A. and Buratowski, S. (1999) The importin/karyopherin Kap1 14 mediates the nuclear import of TATA-binding protein. Proc Natl Acad Sci U S A, 96, 12542-12547.

Mosammaparast, N., Del Rosario, B.C. and Pemberton, L.F. (2005) Modulation of histone deposition by the karyopherin kap114. Mol Cell Biol, 25, 1764-1778. 
Mosammaparast, N., Ewart, C.S. and Pemberton, L.F. (2002a) A role for nucleosome assembly protein 1 in the nuclear transport of histones $\mathrm{H} 2 \mathrm{~A}$ and H2B. EMBO J, 21, 6527-6538.

Mosammaparast, N., Guo, Y., Shabanowitz, J., Hunt, D.F. and Pemberton, L.F. (2002b) Pathways mediating the nuclear import of histones $\mathrm{H} 3$ and $\mathrm{H} 4$ in yeast. $J$ Biol Chem, 277, 862-868.

Mosammaparast, N., Jackson, K.R., Guo, Y., Brame, C.J., Shabanowitz, J., Hunt, D.F. and Pemberton, L.F. (2001) Nuclear import of histone H2A and H2B is mediated by a network of karyopherins. $J$ Cell Biol, 153, 251-262.

Mosammaparast, N. and Pemberton, L.F. (2004) Karyopherins: from nuclear-transport mediators to nuclear-function regulators. Trends Cell Biol, 14, 547-556.

Muhlhausser, P., Muller, E.C., Otto, A. and Kutay, U. (2001) Multiple pathways contribute to nuclear import of core histones. EMBO Rep, 2, 690-696.

Nakielny, S. and Dreyfuss, G. (1999) Transport of proteins and RNAs in and out of the nucleus. Cell, 99, 677-690.

Nakshatri, H., Bhat-Nakshatri, P. and Currie, R.A. (1996) Subunit association and DNA binding activity of the heterotrimeric transcription factor NF-Y is regulated by cellular redox. J Biol Chem, 271, 28784-28791.

Nigg, E.A. (1997) Nucleocytoplasmic transport: signals, mechanisms and regulation. Nature, 386, 779787.

Ornstein, L. (1964) Disc Electrophoresis. I. Background and Theory. Ann N Y Acad Sci, 121, 321-349.

Orphanides, G., Lagrange, T. and Reinberg, D. (1996) The general transcription factors of RNA polymerase II. Genes Dev, 10, 2657-2683.

Orphanides, G. and Reinberg, D. (2002) A unified theory of gene expression. Cell, 108, 439-451.

Ossareh-Nazari, B., Bachelerie, F. and Dargemont, C. (1997) Evidence for a role of CRM1 in signalmediated nuclear protein export. Science, 278, 141-144.

Palacios, I., Hetzer, M., Adam, S.A. and Mattaj, I.W. (1997) Nuclear import of U snRNPs requires importin beta. EMBO J, 16, 6783-6792.

Palmeri, D. and Malim, M.H. (1999) Importin beta can mediate the nuclear import of an arginine-rich nuclear localization signal in the absence of importin alpha. Mol Cell Biol, 19, 1218-1225.

Pante, N. and Kann, M. (2002) Nuclear pore complex is able to transport macromolecules with diameters of about $39 \mathrm{~nm}$. Mol Biol Cell, 13, 425-434.

Paraskeva, E., Izaurralde, E., Bischoff, F.R., Huber, J., Kutay, U., Hartmann, E., Luhrmann, R. and Gorlich, D. (1999) CRM1-mediated recycling of snurportin 1 to the cytoplasm. J Cell Biol, 145, 255-264.

Paschal, B.M. and Gerace, L. (1995) Identification of NTF2, a cytosolic factor for nuclear import that interacts with nuclear pore complex protein p62. J Cell Biol, 129, 925-937. 
Pemberton, L.F., Rosenblum, J.S. and Blobel, G. (1999) Nuclear import of the TATA-binding protein: mediation by the karyopherin Kap114p and a possible mechanism for intranuclear targeting. $J$ Cell Biol, 145, 1407-1417.

Petosa, C., Schoehn, G., Askjaer, P., Bauer, U., Moulin, M., Steuerwald, U., Soler-Lopez, M., Baudin, F., Mattaj, I.W. and Muller, C.W. (2004) Architecture of CRM1/Exportin1 suggests how cooperativity is achieved during formation of a nuclear export complex. Mol Cell, 16, 761-775.

Ploski, J.E., Shamsher, M.K. and Radu, A. (2004) Paired-type homeodomain transcription factors are imported into the nucleus by karyopherin 13. Mol Cell Biol, 24, 4824-4834.

Pollard, V.W., Michael, W.M., Nakielny, S., Siomi, M.C., Wang, F. and Dreyfuss, G. (1996) A novel receptor-mediated nuclear protein import pathway. Cell, 86, 985-994.

Pruss, D., Bartholomew, B., Persinger, J., Hayes, J., Arents, G., Moudrianakis, E.N. and Wolffe, A.P. (1996) An asymmetric model for the nucleosome: a binding site for linker histones inside the DNA gyres. Science, 274, 614-617.

Rexach, M. and Blobel, G. (1995) Protein import into nuclei: association and dissociation reactions involving transport substrate, transport factors, and nucleoporins. Cell, 83, 683-692.

Ribbeck, K. and Gorlich, D. (2001) Kinetic analysis of translocation through nuclear pore complexes. EMBO J, 20, 1320-1330.

Ribbeck, K. and Gorlich, D. (2002) The permeability barrier of nuclear pore complexes appears to operate via hydrophobic exclusion. EMBO J, 21, 2664-2671.

Ribbeck, K., Kutay, U., Paraskeva, E. and Gorlich, D. (1999) The translocation of transportin-cargo complexes through nuclear pores is independent of both Ran and energy. Curr Biol, 9, 47-50.

Ribbeck, K., Lipowsky, G., Kent, H.M., Stewart, M. and Gorlich, D. (1998) NTF2 mediates nuclear import of Ran. EMBO J, 17, 6587-6598.

Richards, S.A., Lounsbury, K.M., Carey, K.L. and Macara, I.G. (1996) A nuclear export signal is essential for the cytosolic localization of the Ran binding protein, RanBP1. J Cell Biol, 134, $1157-1168$.

Robbins, J., Dilworth, S.M., Laskey, R.A. and Dingwall, C. (1991) Two interdependent basic domains in nucleoplasmin nuclear targeting sequence: identification of a class of bipartite nuclear targeting sequence. Cell, 64, 615-623.

Romier, C., Cocchiarella, F., Mantovani, R. and Moras, D. (2003) The NF-YB/NF-YC structure gives insight into DNA binding and transcription regulation by CCAAT factor NF-Y. J Biol Chem, 278, 1336-1345.

Ronchi, A., Berry, M., Raguz, S., Imam, A., Yannoutsos, N., Ottolenghi, S., Grosveld, F. and Dillon, N. (1996) Role of the duplicated CCAAT box region in gamma-globin gene regulation and hereditary persistence of fetal haemoglobin. EMBO J, 15, 143-149.

Rout, M.P. and Aitchison, J.D. (2001) The nuclear pore complex as a transport machine. J Biol Chem, 276, 16593-16596. 
Rout, M.P., Aitchison, J.D., Suprapto, A., Hjertaas, K., Zhao, Y. and Chait, B.T. (2000) The yeast nuclear pore complex: composition, architecture, and transport mechanism. J Cell Biol, 148, 635-651.

Rout, M.P., Blobel, G. and Aitchison, J.D. (1997) A distinct nuclear import pathway used by ribosomal proteins. Cell, 89, 715-725.

Roy, B. and Lee, A.S. (1995) Transduction of calcium stress through interaction of the human transcription factor $\mathrm{CBF}$ with the proximal CCAAT regulatory element of the grp78/BiP promoter. Mol Cell Biol, 15, 2263-2274.

Rupp, R.A., Snider, L. and Weintraub, H. (1994) Xenopus embryos regulate the nuclear localization of XMyoD. Genes Dev, 8, 1311-1323.

Sambrook, J., Fritsch, E.F. and Maniatis, T. (1989) Molecular Cloning: A Laboratory Manual. Cold Spring Harbor Laboratory Press, New York.

Sanger, F., Nicklen, S. and Coulson, A.R. (1977) DNA sequencing with chain-terminating inhibitors. Proc Natl Acad Sci U S A, 74, 5463-5467.

Schlenstedt, G., Smirnova, E., Deane, R., Solsbacher, J., Kutay, U., Gorlich, D., Ponstingl, H. and Bischoff, F.R. (1997) Yrb4p, a yeast ran-GTP-binding protein involved in import of ribosomal protein $\mathrm{L} 25$ into the nucleus. EMBO J, 16, 6237-6249.

Schuller, C. and Ruis, H. (2002) Regulated nuclear transport. Results Probl Cell Differ, 35, 169-189.

Schuster, T., Han, M. and Grunstein, M. (1986) Yeast histone H2A and H2B amino termini have interchangeable functions. Cell, 45, 445-451.

Schwamborn, K., Albig, W. and Doenecke, D. (1998) The histone H1(0) contains multiple sequence elements for nuclear targeting. Exp Cell Res, 244, 206-217.

Schwoebel, E.D., Talcott, B., Cushman, I. and Moore, M.S. (1998) Ran-dependent signal-mediated nuclear import does not require GTP hydrolysis by Ran. J Biol Chem, 273, 35170-35175.

Sharp, P.A., Sugden, B. and Sambrook, J. (1973) Detection of two restriction endonuclease activities in Haemophilus parainfluenzae using analytical agarose--ethidium bromide electrophoresis. Biochemistry, 12, 3055-3063.

Sinha, S., Kim, I.S., Sohn, K.Y., de Crombrugghe, B. and Maity, S.N. (1996) Three classes of mutations in the A subunit of the CCAAT-binding factor CBF delineate functional domains involved in the three-step assembly of the CBF-DNA complex. Mol Cell Biol, 16, 328-337.

Sinha, S., Maity, S.N., Lu, J. and de Crombrugghe, B. (1995) Recombinant rat CBF-C, the third subunit of CBF/NFY, allows formation of a protein-DNA complex with CBF-A and CBF-B and with yeast HAP2 and HAP3. Proc Natl Acad Sci U S A, 92, 1624-1628.

Smith, A., Brownawell, A. and Macara, I.G. (1998) Nuclear import of Ran is mediated by the transport factor NTF2. Curr Biol, 8, 1403-1406.

Stade, K., Ford, C.S., Guthrie, C. and Weis, K. (1997) Exportin 1 (Crm1p) is an essential nuclear export factor. Cell, 90, 1041-1050. 
Steidl, S., Papagiannopoulos, P., Litzka, O., Andrianopoulos, A., Davis, M.A., Brakhage, A.A. and Hynes, M.J. (1999) AnCF, the CCAAT binding complex of Aspergillus nidulans, contains products of the hapB, hapC, and hapE genes and is required for activation by the pathwayspecific regulatory gene amdR. Mol Cell Biol, 19, 99-106.

Steidl, S., Tuncher, A., Goda, H., Guder, C., Papadopoulou, N., Kobayashi, T., Tsukagoshi, N., Kato, M. and Brakhage, A.A. (2004) A Single Subunit of a Heterotrimeric CCAAT-binding Complex Carries a Nuclear Localization Signal: Piggy Back Transport of the Pre-assembled Complex to the Nucleus. J Mol Biol, 342, 515-524.

Stewart, M. (2003) Structural biology. Nuclear trafficking. Science, 302, 1513-1514.

Stewart, M., Kent, H.M. and McCoy, A.J. (1998a) Structural basis for molecular recognition between nuclear transport factor 2 (NTF2) and the GDP-bound form of the Ras-family GTPase Ran. $J$ Mol Biol, 277, 635-646.

Stewart, M., Kent, H.M. and McCoy, A.J. (1998b) The structure of the Q69L mutant of GDP-Ran shows a major conformational change in the switch II loop that accounts for its failure to bind nuclear transport factor 2 (NTF2). J Mol Biol, 284, 1517-1527.

Stoffler, D., Feja, B., Fahrenkrog, B., Walz, J., Typke, D. and Aebi, U. (2003) Cryo-electron tomography provides novel insights into nuclear pore architecture: implications for nucleocytoplasmic transport. J Mol Biol, 328, 119-130.

Stommel, J.M., Marchenko, N.D., Jimenez, G.S., Moll, U.M., Hope, T.J. and Wahl, G.M. (1999) A leucine-rich nuclear export signal in the 553 tetramerization domain: regulation of subcellular localization and p53 activity by NES masking. EMBO J, 18, 1660-1672.

Strahl, B.D. and Allis, C.D. (2000) The language of covalent histone modifications. Nature, 403, 41-45.

Strasser, K., Bassler, J. and Hurt, E. (2000) Binding of the Mex67p/Mtr2p heterodimer to FXFG, GLFG, and FG repeat nucleoporins is essential for nuclear mRNA export. $J$ Cell Biol, 150, 695-706.

Strom, A.C. and Weis, K. (2001) Importin-beta-like nuclear transport receptors. Genome Biol, 2, reviews 3008.

Stuven, T., Hartmann, E. and Gorlich, D. (2003) Exportin 6: a novel nuclear export receptor that is specific for profilin.actin complexes. EMBO J, 22, 5928-5940.

Suzuki, Y., Tsunoda, T., Sese, J., Taira, H., Mizushima-Sugano, J., Hata, H., Ota, T., Isogai, T., Tanaka, T., Nakamura, Y., Suyama, A., Sakaki, Y., Morishita, S., Okubo, K. and Sugano, S. (2001) Identification and characterization of the potential promoter regions of 1031 kinds of human genes. Genome Res, 11, 677-684.

Terns, M.P. and Goldfarb, D.S. (1998) Nuclear transport of RNAs in microinjected Xenopus oocytes. Methods Cell Biol, 53, 559-589.

Titov, A.A. and Blobel, G. (1999) The karyopherin Kap122p/Pdr6p imports both subunits of the transcription factor IIA into the nucleus. J Cell Biol, 147, 235-246. 
Tommasi, S. and Pfeifer, G.P. (1995) In vivo structure of the human cdc2 promoter: release of a p130E2F-4 complex from sequences immediately upstream of the transcription initiation site coincides with induction of cdc2 expression. Mol Cell Biol, 15, 6901-6913.

Truant, R. and Cullen, B.R. (1999) The arginine-rich domains present in human immunodeficiency virus type 1 Tat and Rev function as direct importin beta-dependent nuclear localization signals. Mol Cell Biol, 19, 1210-1217.

Turner, D.L. and Weintraub, H. (1994) Expression of achaete-scute homolog 3 in Xenopus embryos converts ectodermal cells to a neural fate. Genes Dev, 8, 1434-1447.

Verreault, A. (2000) De novo nucleosome assembly: new pieces in an old puzzle. Genes Dev, 14, 14301438.

Verreault, A., Kaufman, P.D., Kobayashi, R. and Stillman, B. (1996) Nucleosome assembly by a complex of CAF-1 and acetylated histones H3/H4. Cell, 87, 95-104.

Vetter, I.R., Arndt, A., Kutay, U., Gorlich, D. and Wittinghofer, A. (1999) Structural view of the RanImportin beta interaction at 2.3 A resolution. Cell, 97, 635-646.

Weber, K. and Osborn, M. (1969) The reliability of molecular weight determinations by dodecyl sulfatepolyacrylamide gel electrophoresis. J Biol Chem, 244, 4406-4412.

Weis, K. (2002) Nucleocytoplasmic transport: cargo trafficking across the border. Curr Opin Cell Biol, 14, 328-335.

Weis, K. (2003) Regulating access to the genome: nucleocytoplasmic transport throughout the cell cycle. Cell, 112, 441-451.

Weis, K., Dingwall, C. and Lamond, A.I. (1996a) Characterization of the nuclear protein import mechanism using Ran mutants with altered nucleotide binding specificities. EMBO J, 15, 71207128.

Weis, K., Ryder, U. and Lamond, A.I. (1996b) The conserved amino-terminal domain of hSRP1 alpha is essential for nuclear protein import. EMBO J, 15, 1818-1825.

Wen, W., Meinkoth, J.L., Tsien, R.Y. and Taylor, S.S. (1995) Identification of a signal for rapid export of proteins from the nucleus. Cell, 82, 463-473.

Wischnewski, J., Rudt, F. and Pieler, T. (2004) Signals and receptors for the nuclear transport of TFIIIA in Xenopus oocytes. Eur J Cell Biol, 83, 55-66.

Wolffe, A. (1998) Chromatin, structure and function. Academic Press, London, UK.

Workman, J.L. and Kingston, R.E. (1998) Alteration of nucleosome structure as a mechanism of transcriptional regulation. Annu Rev Biochem, 67, 545-579.

Wozniak, R.W., Rout, M.P. and Aitchison, J.D. (1998) Karyopherins and kissing cousins. Trends Cell Biol, 8, 184-188.

Xiao, Z., Liu, X. and Lodish, H.F. (2000) Importin beta mediates nuclear translocation of Smad 3. J Biol Chem, 275, 23425-23428. 
Xing, Y., Fikes, J.D. and Guarente, L. (1993) Mutations in yeast HAP2/HAP3 define a hybrid CCAAT box binding domain. EMBO J, 12, 4647-4655.

Yang, Q., Rout, M.P. and Akey, C.W. (1998) Three-dimensional architecture of the isolated yeast nuclear pore complex: functional and evolutionary implications. Mol Cell, 1, 223-234.

Yoshida, K. and Blobel, G. (2001) The karyopherin Kap142p/Msn5p mediates nuclear import and nuclear export of different cargo proteins. J Cell Biol, 152, 729-740.

Zhang, M.J. and Dayton, A.I. (1998) Tolerance of diverse amino acid substitutions at conserved positions in the nuclear export signal (NES) of HIV-1 Rev. Biochem Biophys Res Commun, 243, 113-116.

Zhu, J. and McKeon, F. (1999) NF-AT activation requires suppression of Crm1-dependent export by calcineurin. Nature, 398, 256-260.

Zhu, W., Giangrande, P.H. and Nevins, J.R. (2004) E2Fs link the control of G1/S and G2/M transcription. EMBO J. 


\section{Appendix}

Table I. Importin $\beta$ family members ${ }^{\text {a }}$

\begin{tabular}{|c|c|c|}
\hline mammal/vertebrate & yeast homologs $^{b}$ & known cargoes $^{c}$ \\
\hline Importin $\beta$ & Kap95, Кap $\beta 1 *$ & $\begin{array}{l}\text { ribosomal proteins, core histones, HIV Rev, HIV } \\
\text { Tat, HTLV Rex, SREBP-2, PTHrP, cyclin B1, Smad } \\
\text { proteins, T-cell protein tyrosine phosphatase and } \\
\text { other proteins }\end{array}$ \\
\hline Importin $\beta /$ Importin $\alpha$ & Kap95/Kap60 (SRP1) & $\begin{array}{l}\text { Classical NLS-containing proteins (e.g. SV40 large } \\
\text { T antigen, nucleoplasmin, cyclin E, c-myc, BRCA1, } \\
\text { STAT1) }\end{array}$ \\
\hline Importin $\beta /$ snurportin 1 & Not determined & $m_{3} G$ capped $U$ snRNPs (U snRNA) \\
\hline Importin $\beta / X R I P \alpha$ & & Replication protein A (RPA) \\
\hline Importin $\beta /$ Importin 7 & Not determined & Histone H1 \\
\hline Importin $\beta /$ RanBP8 & Not determined & \\
\hline transportin & Kap104 & $\begin{array}{l}m R N A \text {-binding proteins ( } h n R N P-A 1,-A 2,-F), \\
\text { ribosomal proteins, core histones, TAP, Nab2, Hrp1 }\end{array}$ \\
\hline Transportin2 & & $H u R$ \\
\hline TransportinSR & Kap111, Mtr10 & $\begin{array}{l}\text { Proteins with SR domains, mRNA-binding proteins, } \\
\text { Npl3, Hrp1 }\end{array}$ \\
\hline Importin 4 & Kap123, Yrb4 & Core histones, ribosomal proteins, SRP proteins \\
\hline Importin 5 & Kap121, Pse1 * & $\begin{array}{l}\text { Core histones, ribosomal proteins, Pho4, Ste12, } \\
\text { Spo12, Pdr1, Yap1, Aft1, SRP proteins, core } \\
\text { histones, ribosomal proteins }\end{array}$ \\
\hline Importin 7 & Kap119, Nmd5 & $\begin{array}{l}\text { HIV RTC, Histone H1 (importin } \beta / 7 \text { dimer), GR, } \\
\text { core histones, ribosomal proteins, TFIIS, Hog1, } \\
\text { MAPK, Crz1, Ssa4 }\end{array}$ \\
\hline Importin 8 & & SRP19 \\
\hline Importin 9 & Kap114 & $\begin{array}{l}\text { Core histones, ribosomal proteins, TBP, core } \\
\text { histones H2A and H2B, Nap1 }\end{array}$ \\
\hline Importin 11 & (Lhp2) Kap120 & UbcM2, rpL12 \\
\hline Importin 13 & & $\begin{array}{l}\text { Import: RBM8 (MGN), Ubc9, Pax6, Pax3, Crx } \\
\text { export: eIF-1A }\end{array}$ \\
\hline Crm1/exportin1 & Crm1/Xpo1/Kap124* & $\begin{array}{l}\text { Leucine-rich NES containing proteins (e.g. HIV-1 } \\
\text { Rev, HTLV-1 Rex, PKI, MAPKK, cyclin B1, I B } \alpha \text {, } \\
\text { p53, HDM2, Yap1, Pap1), Snurportin1, } m_{7} G\end{array}$ \\
\hline
\end{tabular}




\begin{tabular}{|c|c|c|}
\hline & & $\begin{array}{l}\text { capped U snRNAs, viral mRNAs, } 5 S \text { rRNA (possibly } \\
\text { mediated by TFIIIA or rpL5)) }\end{array}$ \\
\hline$C A S$ & Cse1/Kap109* & Importin $\alpha$ \\
\hline exportin- $t$ & Los1/Kap127 & $t R N A$ \\
\hline exportin 4 & Not determined & $e I F-5 A$ \\
\hline exportin 5 & Kap142, Msn5 & $\begin{array}{l}I L F, \text { eEF-1A, precursors of miRNA, } t R N A, \\
\text { minihelix RNA } \\
\text { Export: phosphorylated proteins: Pho4, Far1, Mig1, } \\
\text { Cdh1, Rtg1, Rtg3, Crz1, Msn2, Msn4, Swi6 } \\
\text { import: Rpa complex }\end{array}$ \\
\hline exportin 6 & & Profilin/actin \\
\hline \multirow[t]{3}{*}{ exportin 7} & & p50RhoGAP, 14-3-38, eIF1 and other proteins \\
\hline & Kap108, Sxm1 & Probably ribosomal proteins, Lhp1 \\
\hline & Kap122, Pdr6 & TFIIA (Toa1, Toa2) \\
\hline \multicolumn{3}{|l|}{ RanBP6 } \\
\hline RanBP17 & & \\
\hline
\end{tabular}

${ }^{a}$ Derived from (Chook and Blobel, 2001; Gorlich and Kutay, 1999; Harel and Forbes, 2004; Jans et al., 2000; Kim, 2004; Lund et al., 2004; Mingot et al., 2004; Mosammaparast and Pemberton, 2004; Nakielny and Dreyfuss, 1999; Ploski et al., 2004; Strom and Weis, 2001)

$\mathrm{b}$ sequence, not functional, homologs

${ }^{\mathrm{c}}$ cargoes identified in vertebrates are shown in italics (some of them have been also confirmed as cargoes in yeast) Alternative names for each transport receptor (karyopherin) are publicized and asterisks $(*)$ indicate karyopherins encoded by essential genes in yeast. 
Table II. List of expression constructs used in this study

\begin{tabular}{|c|c|c|c|c|}
\hline name & protein (aa) & primer / DNA fragment (template) & vector & restriction sites \\
\hline pJK1 & NF-YB (1-204) & pMB101 Sma-I / Nhe-I & pQE70_zz & EcoR-V / Spe-I \\
\hline pJK2 & NF-YC (1-334) & pMB105 Sma-I / Nhe-I & pQE70_zz & EcoR-V / Spe-I \\
\hline pJK3 & H2A-d (1-26) & pMB10 Sma-I / Nhe-I & pQE70_6z_fl & EcoR-V / Spe-I \\
\hline pJK4 & H2A-d (26-129) & pMB48 Sma-I / Nhe-I & pQE70_6z_fl & EcoR-V / Spe-I \\
\hline pJK5 & H2A-d (1-26) & pMB10 Sma-I / Nhe-I & pQE70_zz_fl & EcoR-V / Spe-I \\
\hline pJK6 & H2A-d (26-129) & pMB48 Sma-I / Nhe-I & pQE70_zz_fl & EcoR-V / Spe-I \\
\hline pJK7 & H2B-d (1-35) & pWA364 Sma-I / Nhe-I & pQE70_zz_fl & EcoR-V / Spe-I \\
\hline pJK8 & H2B-d (36-125) & pWA365 Sma-I / Nhe-I & pQE70_zz_fl & EcoR-V / Spe-I \\
\hline pJK9 & H2B-d (1-35) & pWA364 Sma-I / Nhe-I & pQE70 6z fl & EcoR-V / Spe-I \\
\hline pJK10 & H2B-d (36-125) & pWA365 Sma-I / Nhe-I & pQE70_6z_fl & EcoR-V / Spe-I \\
\hline pJK11 & NF-YB (1-204) & pMB101 Sma-I / Nhe-I & pQE70_6z_fl & EcoR-V / Spe-I \\
\hline pJK12 & NF-YC (1-334) & pMB105 Sma-I / Nhe-I & pQE70_6z_fl & EcoR-V / Spe-I \\
\hline pJK13 & NF-YB (56-139) & pMB103 Sma-I / Nhe-I & pQE70_6z_fl & EcoR-V / Spe-I \\
\hline pJK14 & NF-YC (42-119) & pMB107 Sma-I / Nhe-I & pQE70_6z_fl & EcoR-V / Spe-I \\
\hline pJK15 & H3-k (1-61) & OJK1 / OJK2 & pKS10 & Bgl-II / Nhe-I \\
\hline pJK16 & H3-k (61-136) & OJK3 / OJK4 & pKS10 & Bgl-II / Nhe-I \\
\hline pJK17 & H4-j (1-27) & OJK5 / OJK6 & pKS10 & Bgl-II / Nhe-I \\
\hline pJK18 & H4-j (27-104) & OJK7 / OJK8 & pKS10 & Bgl-II / Nhe-I \\
\hline pJK19 & NF-YB (1-204) & OJK11 / OJK12 & $\mathrm{pCS} 2+\mathrm{MT}$ & EcoR-I / Stu-I \\
\hline pJK20 & NF-YC (1-334) & OJK13 / OJK14 & $\mathrm{pCS} 2+\mathrm{MT}$ & EcoR-I / Stu-I \\
\hline pJK21 & H3-k (1-61) & pJK15 Sma-I / Nhe-I & pQE70_zz_fl & EcoR-V / Spe-I \\
\hline pJK22 & H3-k (1-61) & pJK15 Sma-I / Nhe-I & pQE70_6z_fl & EcoR-V / Spe-I \\
\hline pJK23 & H3-k (61-135) & pJK16 Sma-I / Nhe-I & pQE70_zz_fl & EcoR-V / Spe-I \\
\hline pJK24 & H3-k (61-135) & pJK16 Sma-I / Nhe-I & pQE70_6z_fl & EcoR-V / Spe-I \\
\hline pJK25 & H4-j (1-27) & pJK17 Sma-I / Nhe-I & pQE70_zz_fl & EcoR-V / Spe-I \\
\hline pJK26 & H4-j (1-27) & pJK17 Sma-I / Nhe-I & pQE70_6z_fl & EcoR-V / Spe-I \\
\hline pJK27 & H4-j (27-102) & pJK18 Sma-I / Nhe-I & pQE70_zz_fl & EcoR-V / Spe-I \\
\hline pJK28 & H4-j (27-102) & pJK18 Sma-I / Nhe-I & pQE70_6z_fl & EcoR-V / Spe-I \\
\hline pJK31 & H2B-d (1-125) & pWA352 Sma-I / Nhe-I & pQE70_zz_fl & EcoR-V / Spe-I \\
\hline pJK32 & H3-k (1-135) & pWA356 Sma-I / Nhe-I & pQE70_zz_fl & EcoR-V / Spe-I \\
\hline pJK33 & H4-j (1-102) & pWA357 Sma-I / Nhe-I & pQE70_zz_fl & EcoR-V / Spe-I \\
\hline pJK34 & NF-YA (1-346) & OJK9 / OJK10 & pQE70_zz_fl & EcoR-V / Spe-I \\
\hline pJK35 & NF-YA (1-346) & OJK9 / OJK10 & pQE70_6z_fl & EcoR-V / Spe-I \\
\hline pJK38 & NF-YA (1-346) & OJK17 / OJK18 & pKS10 & Bgl-II / Nhe-I \\
\hline pJK45 & p15A ori (pACYC177) & Pst-1 / Nru-1 & pET21b & Pst-1 / PshA1 \\
\hline pJK46 & NF-YB (1-204) & OJK74 / OJK26 & pJK45 & Nde-1 / Sac-1 \\
\hline pJK47 & NF-YC (1-334) & OJK27 / OJK28 & pET41a & Spe-1 / Sac-1 \\
\hline pJK48 & NF-YA (1-346) & OJK29 / OJK30 & pJK45 & Nde-1 / Sac-1 \\
\hline pJK49 & H2A-d (37-129) & pWA363 Sma-I / Nhe-I & pQE70_zz_fl & EcoR-V / Spe-I \\
\hline pJK50 & H2A-d (37-129) & pWA363 Sma-I / Nhe-I & pQE70_6z_fl & EcoR-V / Spe-I \\
\hline pJK51 & NF-YA (1-181) & OJK17 / OJK34 & pKS10 & Bgl-II / Nhe-I \\
\hline pJK52 & NF-YA (182-346) & OJK35 / OJK18 & pKS10 & Bgl-II / Nhe-I \\
\hline
\end{tabular}




\begin{tabular}{|c|c|c|c|c|}
\hline pJK53 & NF-YA (1-261) & OJK17 / OJK41 & pKS10 & Bgl-II / Nhe-I \\
\hline pJK54 & NF-YA (262-346) & OJK36 / OJK18 & pKS10 & Bgl-II / Nhe-I \\
\hline pJK55 & NF-YA (262-317) & OJK36 / OJK38 & pKS10 & Bgl-II / Nhe-I \\
\hline pJK56 & NF-YA (262-282) & OJK36 / OJK37 & pKS10 & Bgl-II / Nhe-I \\
\hline pJK57 & NF-YA (273-296) & OJK39 / OJK40 & pKS10 & Bgl-II / Nhe-I \\
\hline pJK61 & H2A-d (1-129) & OJK46 / OJK47 & pET41a & EcoR-I / Not-I \\
\hline pJK63 & H2B-d (1-126) & OJK50 / OJK51 & pET41a & EcoR-I / Not-I \\
\hline pJK65 & H2A-d (1-26) & OJK54 / OJK61 & pGEX 4T-1 & BamH-I / Sma-I \\
\hline pJK66 & BIB (32-74) & OMW38 / OMW39 & pQE70_zz_fl & EcoR-V / Spe-I \\
\hline pJK67 & NF-YA (297-317) & OJK56 / OJK38 & pKS10 & Bgl-II / Nhe-I \\
\hline pJK68 & NF-YA (262-296) & OJK36 / OJK40 & pKS10 & Bgl-II / Nhe-I \\
\hline pJK69 & NF-YA (283-317) & OJK57 / OJK38 & pKS10 & Bgl-II / Nhe-I \\
\hline pJK70 & H2B-d (1-126) & OJK58 / OJK59 & pGEX 4T-1 & BamH-I / Sma-I \\
\hline pJK78 & NF-YA (283-296) & OJK57 / OJK40 & pKS10 & Bgl-II / Nhe-I \\
\hline pJK79 & NF-YA (1-282) & OJK17 / OJK37 & pKS10 & Bgl-II / Nhe-I \\
\hline pJK84 & NF-YA $\Delta 262-282$ & OJK75 / OJK86 & pJK 53 & Nhe-I / Nru-I \\
\hline pJK85 & NF-YA $\Delta$ 262-297 & OJK71 / OJK86 & pJK 53 & Nhe-I / Nru-I \\
\hline pJK86 & NF-YA $\Delta 262-317$ & OJK73 / OJK86 & pJK 53 & Nhe-I / Nru-I \\
\hline pJK87 & NF-YA $\Delta$ 282-297 & OJK71 / OJK86 & pJK 79 & Nhe-I / Nru-I \\
\hline pJK88 & NF-YA $\Delta 282-317$ & OJK73 / OJK86 & pJK 79 & Nhe-I / Nru-I \\
\hline pJK95 & NF-YA (1-346) & OJK86B / OJK87 & pGEX 4T-1 & EcoR-1 / Xho-1 \\
\hline pJK96 & NF-YB (1-204) & OJK88 / OJK89 & pGEX 4T-1 & BamH-I / Sma-I \\
\hline pJK97 & NF-YC (1-334) & OJK90 / OJK91 & pGEX 4T-1 & BamH-I / Sma-I \\
\hline pJK100 & NF-YA (1-296) & OJK17 / OJK40 & pKS10 & Bgl-II / Nhe-I \\
\hline pJK102 & NF-YA (283-346) & OJK75 / OJK72 OJK86 & pKS10 & Nhe-I / Nru-I \\
\hline pJK103 & NF-YA K269A & OJK17/OJK100 \& OJK96/ OJK18 & pKS10 & Bgl-II / Nhe-I \\
\hline pJK104 & NF-YA KRR276_278NLG & OJK17/OJK101 \& OJK97/ OJK18 & pKS10 & Bgl-II / Nhe-I \\
\hline pJK105 & NF-YA RRK294_296LGQ & OJK17/OJK102 \& OJK98/ OJK18 & pKS10 & Bgl-II / Nhe-I \\
\hline pJK106 & NF-YA RKR309-311LAG & OJK17/OJK103 \& OJK99/ OJK18 & pKS10 & Bgl-II / Nhe-I \\
\hline pJK108 & NF-YA (1-346) & OJK106/ OJK107 (template pJK38) & egfp_lca1_pcdna3 & Xho-I / Apa-I \\
\hline pJK114 & NF-YA (1-346) & OJK114 neu / OJK115 neu & pcHA2 & EcoR-1 / Hind-III \\
\hline pJK115 & NF-YB (1-204) & OJK116 / OJK117 & pcHA2 & BamH-I / EcoR-1 \\
\hline pJK116 & NF-YC (1-334) & OJK118 / OJK119 & pcHA2 & BamH-I / EcoR-1 \\
\hline pJK120 & NF-YB (1-55) & OJK88 / OJK123 & pGEX 4T-1 & BamH-I / Sma-I \\
\hline pJK121 & NF-YB (56-151) & OJK124 / OJK125 & pGEX 4T-1 & BamH-I / Sma-I \\
\hline pJK122 & NF-YB (152-204) & OJK126 / OJK89 & pGEX 4T-1 & BamH-I / Sma-I \\
\hline pJK123 & NF-YC (1-23) & OJK90 / OJK127 & pGEX 4T-1 & BamH-I / Sma-I \\
\hline pJK124 & NF-YC (24-119) & OJK128 / OJK129 & pGEX 4T-1 & BamH-I / Sma-I \\
\hline pJK125 & NF-YC (120-334) & OJK130 / OJK91 & pGEX 4T-1 & BamH-I / Sma-I \\
\hline pJK130 & pJK104/105 (pJK104 template) & OJK17/OJK102 \& OJK98/ OJK18 & pKS10 & Bgl-II / Nhe-I \\
\hline pJK131 & pJK104/106 (pJK106 template) & OJK17/OJK101 \& OJK97/ OJK18 & pKS10 & Bgl-II / Nhe-I \\
\hline pJK132 & NF-YB (1-204) & OJK131 / OJK132 & egfp_lca1_pcdna3 & Xho-I / Apa-I \\
\hline pJK133 & NF-YC (1-334) & OJK133 / OJK134 & egfp_lca1_pcdna3 & Xho-I / Apa-I \\
\hline pJK143 & NF-YA (1-346) & OJK139 / OJK140 & $\mathrm{pCS} 2+\mathrm{MT}$ & EcoR-I / Stu-I \\
\hline
\end{tabular}


Appendix

\begin{tabular}{|l|l|l|l|l|}
\hline pJK154 & NF-YA $\Delta$ 262-317 & OJK139 / OJK140 (pJK86 template) & pCS2+MT & EcoR-I / Stu-I \\
\hline pJK155 & pJK130 & OJK139 / OJK140 (pJK130 template) & pCS2+MT & EcoR-I / Stu-I \\
\hline pJK161 & NF-YB (1-206) & OJK156 / OJK157 (pjk90 template) & pCS2+MT & EcoR-I / Stu-I \\
\hline pJK162 & NF-YC (1-334) & OJK13 / OJK157 (pjk91 template) & pCS2+MT & EcoR-I / Stu-I \\
\hline pJK163 & NF-YB (1-204) & OJK158 / OJK159 (pjk11 template) & pCS2+MT & EcoR-I / Stu-I \\
\hline pJK164 & NF-YC (1-334) & OJK158 / OJK160 (pjk12 template) & pCS2+MT & EcoR-I / Stu-I \\
\hline pJK169 & EGFP & OJK163 / OJK164 & pGEX 4T-1 & BamH-I / EcoR-I \\
\hline pJK180 & X.Pax6 & pJK170 EcoR-I / Xho-I & pJK169 & EcoR-I / Xho-I \\
\hline pJK197 & YA K269A & OJK86B / OJK87 (pJK103 template) & pGEX 4T-1 & EcoR-1/ Xho-1 \\
\hline pJK198 & YA KRR276_278NLG & OJK86B / OJK87 (pJK104 template) & pGEX 4T-1 & EcoR-1/ Xho-1 \\
\hline pJK199 & YA RRK294_296LGQ & OJK86B / OJK87 (pJK105 template) & pGEX 4T-1 & EcoR-1 / Xho-1 \\
\hline pJK200 & YA RKR309-311LAG & OJK86B / OJK87 (pJK106 template) & pGEX 4T-1 & EcoR-1 / Xho-1 \\
\hline pJK201 & pJK104/105 & OJK86B / OJK87 (pJK130 template) & pGEX 4T-1 & EcoR-1 / Xho-1 \\
\hline pJK202 & pJK104/106 & OJK86B / OJK87 (pJK131 template) & pGEX 4T-1 & EcoR-1 / Xho-1 \\
\hline pJK203 & NF-YA (1-269) & OJK86B / OJK87 (accidental stop) & pGEX 4T-1 & EcoR-1 / Xho-1 \\
\hline
\end{tabular}




\section{Danksagung}

Der mit Abstand am schwierigsten zu schreibende Abschnitt einer Doktorarbeit ist die Danksagung, denn hier kann man nichts mehr erreichen, außer Unbeliebtheit bei den Nichterwähnten. Angesichts der vielfältigen Hilfe und Unterstützung, die einem innerhalb des Zeitraums der Doktorarbeit zu Teil werden, ist es eher unmöglich seinen Dank, in wenige Worte gefasst, zum Ausdruck zu bringen. Daher möchte ich zunächst allen recht, recht herzlich danken, die mich auf dem dunklen steinigen Pfad der Promotion begleitet haben und diesen mit Licht erfüllten. Dank Euch werde ich die Zeit in Göttingen letztendlich immer als äußerst angenehm in Erinnerung behalten.

Im Einzelnen möchte ich natürlich Ihnen Herr Doenecke und Dir Werner dafür danken, (i) mir die Möglichkeit gegeben zu haben als „Fachfremder“ diese Arbeit am Institut durchzuführen, (ii) mir immer mit Sachverstand zur Seite gestanden zu haben, und (iii) mir die nötigen experimentellen Freiheiten gelassen zu haben (Vielen Dank und wir sehen uns in „Wahl“!!).

Außerdem bedanke ich mich bei Ihnen Herr Ficner für die spontane Bereitschaft das Korreferat zu übernehmen. Ich weiß, dass es keine Selbstverständlichkeit ist, diese Aufgabe so kurzfristig zu übernehmen.

Darüber hinaus danke ich natürlich allen Mitarbeitern/Mitarbeiterinnen der Abteilung Doenecke für die super Zusammenarbeit, das ausgesprochen harmonische Arbeitsklima, und vor allem Euch Christa, Kristina und Diana für die Aufrechterhaltung des täglichen Laborbetriebs sowie für die nötige Gelassenheit, über meine geistesarmen Sprüche hinwegzusehen. Gleiches trifft natürlich insbesondere auch auf Dich liebe Sonja zu, da Du als Laborkollegin mit Abstand am meisten unter mir zu leiden hattest. Ich hoffe Du verzeihst mir, und ich danke Dir für die äußerst angenehme Zeit im und außerhalb des Labors.

Außerdem möchte ich mich an dieser Stelle bei allen Mitarbeitern/Mitarbeiterinnen der Abteilung Pieler für die mir entgegengebrachte Gastfreundschaft und Herzlichkeit bedanken. Mein spezieller Dank gilt natürlich Ihnen Herr Pieler für die Bereitstellung der Räumlichkeiten und für Ihre Offenheit gegenüber Fragen meinerseits. Des Weiteren möchte ich mich bei Euch Susanne und Katja recht herzlich bedanken, und zwar nicht nur für die mehrfachen Präparationen der Oozyten und die Lehrstunden bezüglich Mikroinjektionen, sondern auch dafür mir immer mit Rat und Tat zur Seite gestanden zu haben. Außerdem gilt mein besonderer Dank den vier Musketieren Andreas, Martin, Marco und Tiemo, die es auf wundervolle Art und Weise verstehen, Arbeit mit Vergnügen zu kombinieren, und mir, wahrscheinlich leider vergeblich, versucht haben beizubringen, wie man alles richtig macht (Vielen Dank und die nächste Runde geht auf mich!). 
Hervorheben muss ich allerdings an dieser Stelle noch mal Andreas und seine ganze Familie. Abgesehen von den unzähligen Sequenzierungen die Du im Laufe der Zeit für mich erledigt hast, danke ich Dir natürlich herzlichst für die breite Unterstützung, die Du mir über die letzten Jahre in allen Belangen des Lebens geboten hast (Ich hoffe, es hat Dich nicht zu viele Haare gekostet!).

Wichtige Bezugspersonen für mich im und außerhalb des Graduiertenkollegs waren Constanæe, Alex und Katrin, wobei ich natürlich Dir Katrin recht herzlich für die Hilfe bezüglich der Arbeit mit Hefe danken möchte. Wer weiß, vielleicht ergibt sich ja doch noch mal eine Zusammenarbeit.

Weiterhin danke ich allen Mitarbeitern/Mitarbeiterinnen der Abteilung Haucke für deren Unterstützung bei meiner Laborarbeit. Insbesondere möchte ich mich bei Euch Nadja und Micha für die „Bereitstellung“ Eurer fachlichen Kompetenz und der vielen Plasmide bedanken (irgendwie vermisse ich Euch schon).

Zu guter letzt, möchte ich mich an dieser Stelle bei Dir Ralf für dein stetes Interesse am Fortgang meiner Arbeit und die damit verbundenen abendlichen Gesprächsrunden bedanken. Es ist eine Freude gewesen, deinen Ausführungen zu lauschen. Es hat mein Wissen und Verständnis auf mannigfaltige Art und Weise bereichert und mich darüber hinaus angeregt, über so manche Dinge im Leben intensiver nachzudenken (Vielen Dank und du bist Tag und Nacht willkommen!). 


\section{Curriculum Vitae}

Name:

Kahle

Vorname:

Jörg

Geburtsdatum:

10.01.1975

Geburtsort:

Dresden

Staatsangehörigkeit:

deutsch

$1981-1983$

84. Oberschule; Dresden

$1983-1990$

10. POS; Dresden

$1990-1991$

11. Oberschule; Dresden

$1991-1993$

Gymnasium Dresden-Altstadt; Dresden

$6 / 1993$

$7 / 1993-10 / 1994$

Abschluss: allgemeine Hochschulreife

$1994-1995$

$10 / 1995$

9/1997

$9 / 1999$

Zivildienst im Krankenhaus Dresden-Friedrichstadt; Dresden

Auslandsaufenthalt, Navarro College; Corsicana, Texas, USA

Beginn des Studiums der Lebensmittelchemie an der TU Dresden

Vordiplom in den Fächern: Anorganische Chemie, Organische

Chemie, Physikalische Chemie, Physik und Allgemeine Biologie

Diplomprüfung in den Fächern: Lebensmittelchemie,

Lebensmitteltechnologie, Lebensmitteltoxikologie,

Ernährungslehre und Mikrobiologie

$1 / 2000-6 / 2000$

Diplomarbeit am Institut für Lebensmittelchemie, TU Dresden, bei

Prof. Dr.-Ing. T. Henle; Thema: „Untersuchungen zur Reaktivität proteingebundener Lysin- und Argininreste im Verlauf fortgeschrittener nichtenzymatischer Glycosylierungreaktionen“

$7 / 2000$ Abschluss: Diplom (Lebensmittelchemiker)

$10 / 2000-9 / 2001$

$9 / 2001$

Landesuntersuchungsanstalt für das Gesundheits- und Veterinärwesen Sachsen, Standort Chemnitz

$10 / 2001-3 / 2005$

Abschluss: Staatlich geprüfter Lebensmittelchemiker

Doktorarbeit am Institut für Biochemie und Molekulare

Zellbiologie der Georg-August-Universität Göttingen in der Abteilung Molekularbiologie, bei Prof. Dr. D. Doenecke

Thema: „Mechanisms underlying the nuclear transport of histones and histone-related proteins“ 